
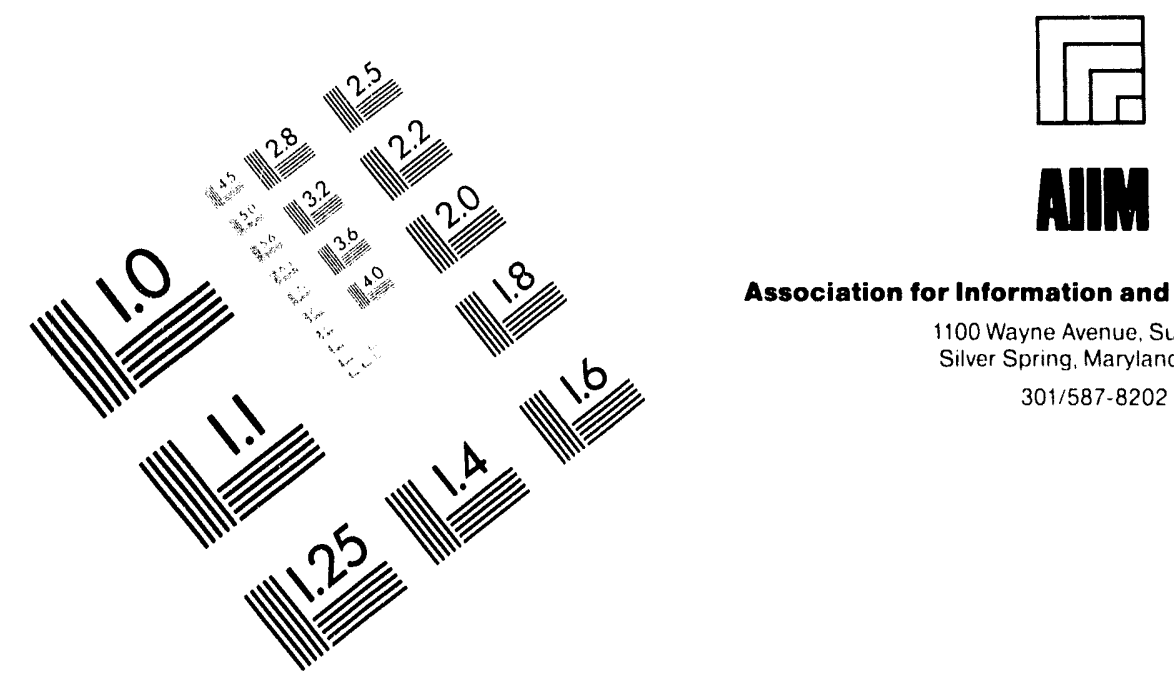

Association for Information and Image Management

1100 Wayne Avenue. Suite 1100

Silver Spring. Maryland 20910

301/587-8202

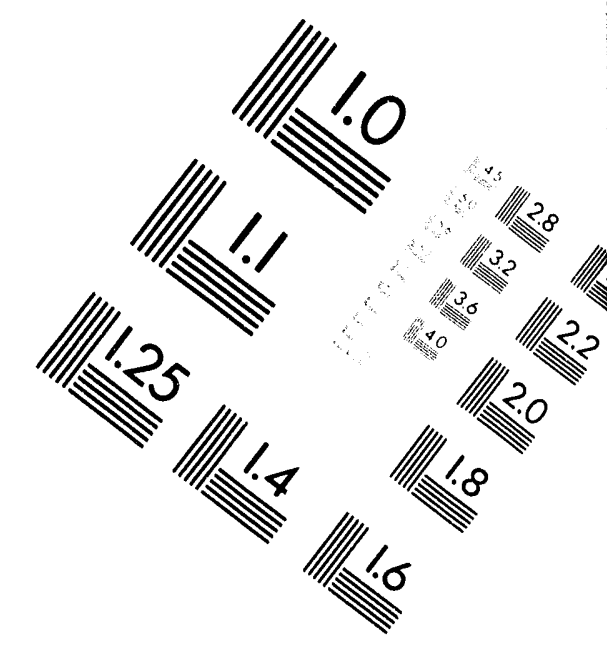

Centimeter

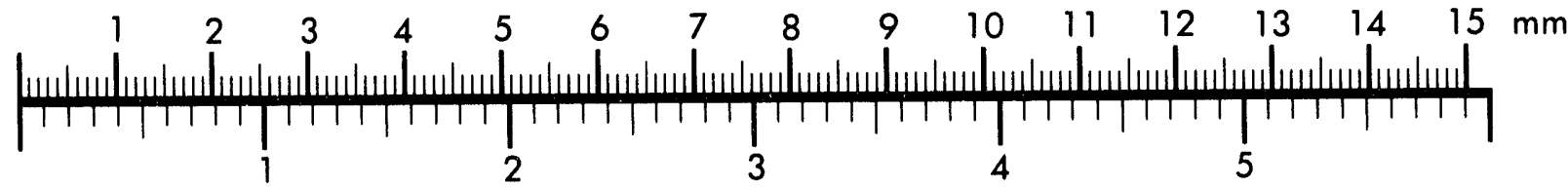

Inches
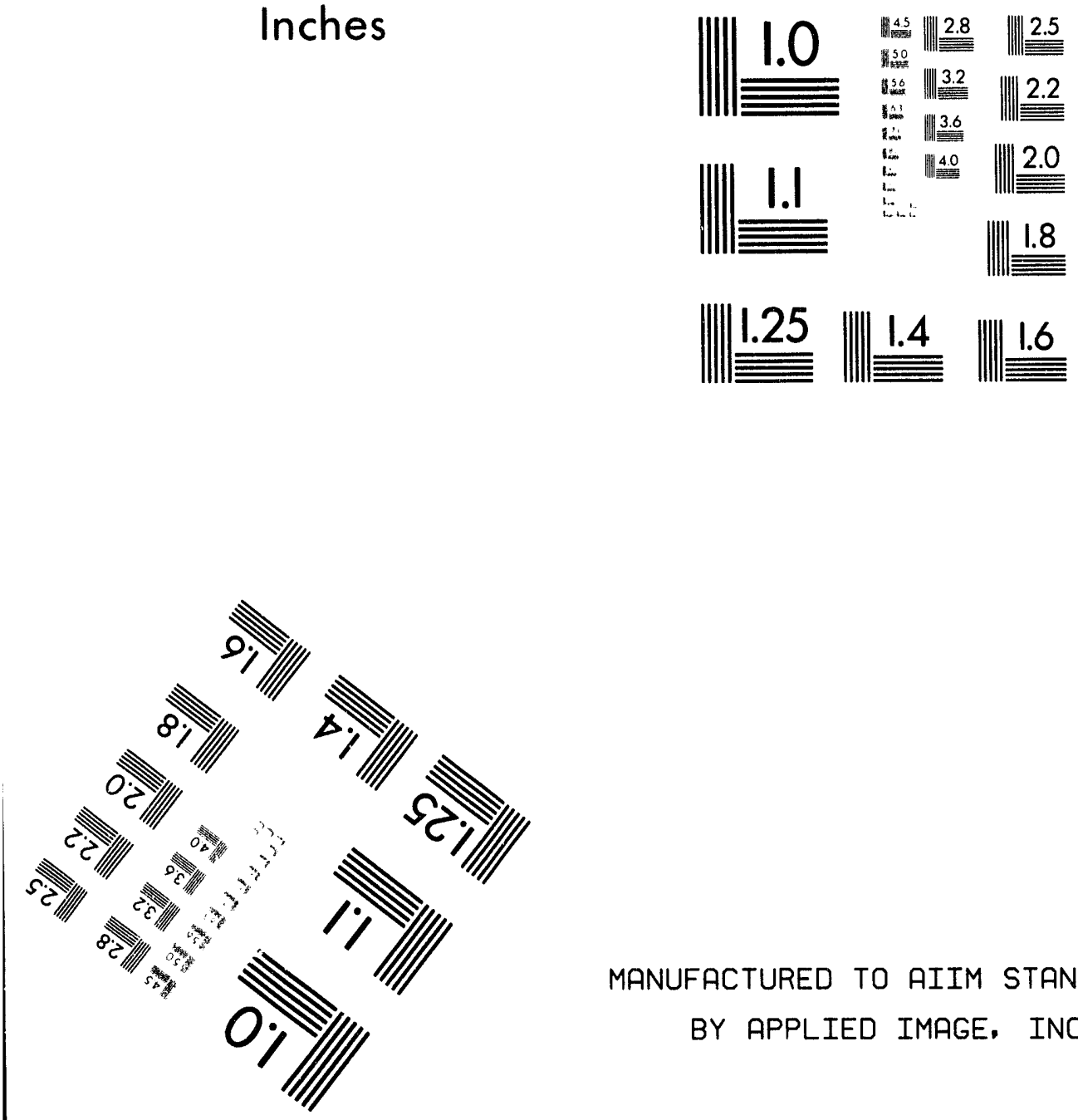

MANUFACTURED TO AIIM STANDARDS

BY APPLIED IMAGE, INC.

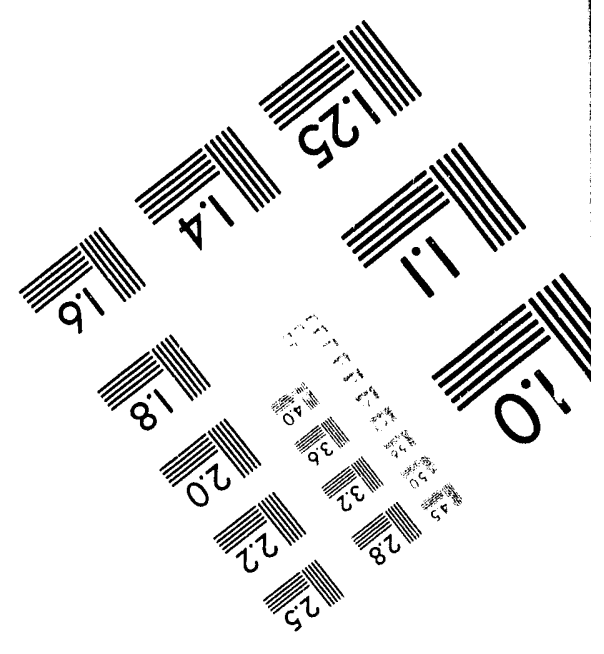



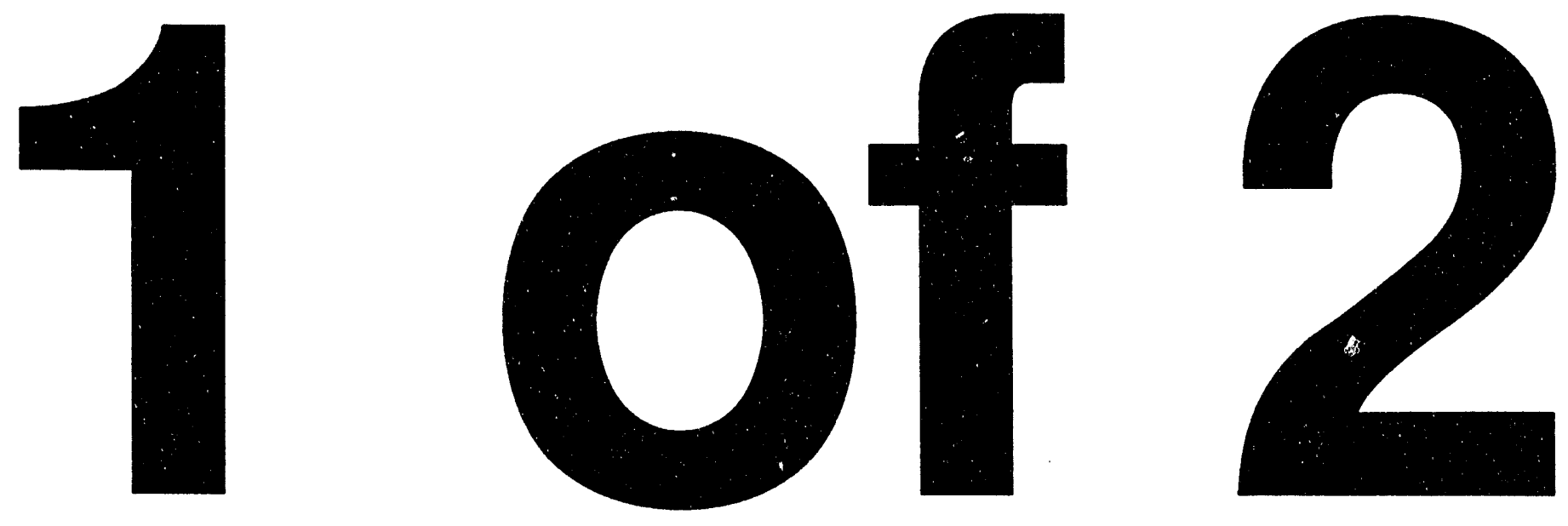
Engineering Physics and Mathematics Division

\title{
SCOPING CALCULATIONS OF POWER SOURCES FOR NUCLEAR ELECTRIC PROPULSION
}

\author{
F. C. Difilippo
}

Date Published: May 1994

Research sponsored by the National Aeronautics and Space Administration

Prepared by the

OAK RIDGE NATIONAL LABORATORY

Oak Ridge, Tennessee 37831 managed by

MARTIN MARIETTA ENERGY SYSTEMS, INC.

for the

U.S. Department of Energy

under contract DE-AC05-84OR21400 


\section{TABLE OF CONTENTS}

ACKNOWLEDGEMENTS $\ldots \ldots \ldots \ldots \ldots \ldots \ldots \ldots \ldots \ldots \ldots \ldots \ldots$

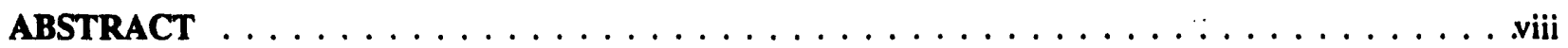

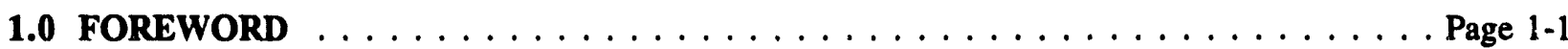

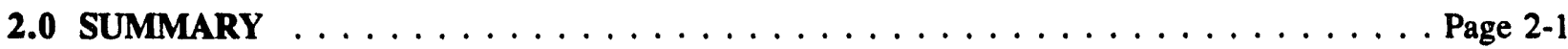

3.0 INTRODUCTION $\ldots \ldots \ldots \ldots \ldots \ldots \ldots \ldots \ldots \ldots \ldots \ldots$ Page 3-1

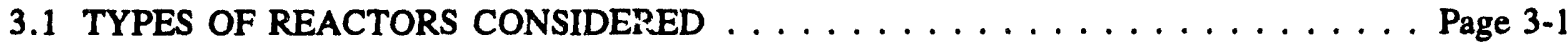

4.0 NEUTRONIC CALCULATIONS $\ldots \ldots \ldots \ldots \ldots \ldots \ldots \ldots \ldots \ldots \ldots$ Page 4-1 $\ldots \ldots \ldots \ldots$

4.1 NERVA DERIVATIVE DATA BASE . . . . . . . . . . . . . . Page 4-1

4.1.1 Neutronic Properties of the Core . . . . . . . . . . . . . . . . . Page 4-1

4.1.2 Reactivity Worth of Beryllium Reflector . . . . . . . . . . . . . Page 4-1

4.1.3 Reactivity Worth of Burn-Up . . . . . . . . . . . . . . . Page 4-1

4.1.4 Depletion of ${ }^{235} \mathrm{U} \ldots \ldots \ldots \ldots \ldots \ldots \ldots$ Page 4-1

4.1.5 Reactivity Worth of $B_{4} C$ Sheet $\ldots \ldots \ldots \ldots$ Page 4-2

4.2 FUEL PIN DATABASE $\ldots \ldots \ldots \ldots \ldots \ldots \ldots \ldots$ Page $4-10$

4.2.1 Neutronic Properties of the Core . . . . . . . . . . . . . . Page 4-10

4.2.2 Reactivity Worth of Beryllium Oxide Reflector . . . . . . . . . . . Page 4-10

4.2.3 Reactivity Worth of Burnup and Isotopics . . . . . . . . . . . Page 4-10

4.2.4 Reactivity Worth of the Pressure Vessel . . . . . . . . . . . . . Page 4-15

4.2.5 Reactivity worth of $\mathrm{B}_{4} \mathrm{C}$ sheets $\ldots \ldots \ldots \ldots \ldots$ Page 4-16

5.0 SHIELdING CALCULATIONS $\ldots \ldots \ldots \ldots \ldots \ldots \ldots$ Page 5.1

5.1 NEUTRON SHIELDING $\ldots \ldots \ldots \ldots \ldots \ldots \ldots \ldots$ Page $5-1$

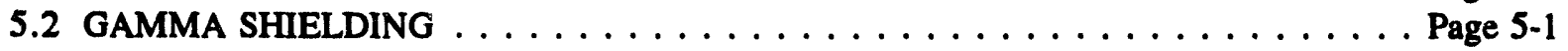

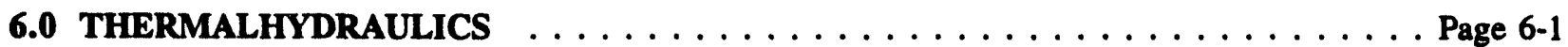

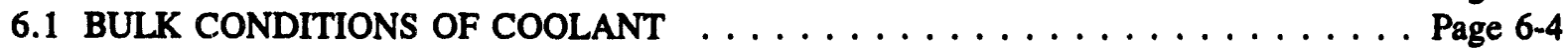

6.1 .1 Bulk Conditions of the Helium Coolant . . . . . . . . . . . . Page 6-4

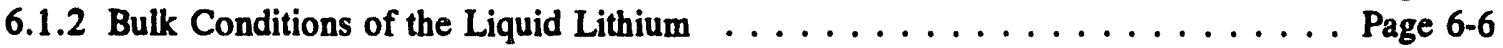

6.2 WALL TEMPERATURE . . . . . . . . . . . . . . . . . . Page 6-6

6.2.1 Wall Temperature of the NERVA Derivative Fuel Element . . . . . . . . . Page 6-7

6.2.2 Wall Temperature of the Fuel Rods . . . . . . . . . . . . . Page 6-7

6.3 FROM WALL TO FUEL TEMPERATURES . . . . . . . . . . . . . Page 6-7

6.3.1 Fuel Temperature of the NERVA Derivative Reactor $\ldots \ldots \ldots \ldots$. . . Page 6-7

6.3.2 Fuel Temperature of the Fuel Pin Reactor . . . . . . . . . . . . . Page 6-8

6.4 THERMALHYDRAULIC FEEDBACK VIA THE PRESSURE VESSEL . . . . . . Page 6-8

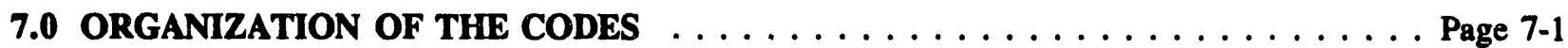

7.1 SELECTION AND CALCULATION OF A DESIGN $\ldots \ldots \ldots \ldots \ldots$ Page $7-1$

7.2 DESIGN OF THE CONTROL DRUMS $\ldots \ldots \ldots \ldots \ldots \ldots$ Page $7-2$

7.3 SOME DETAILS OF THE CODE NEPNERVA $\ldots \ldots \ldots \ldots \ldots \ldots$ Page $7-3$

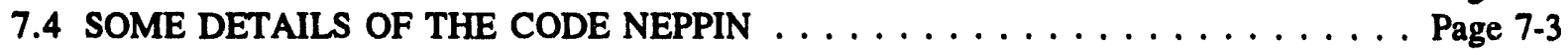

8.0 INPUT AND OUTPUT DESCRIPTIONS $\ldots \ldots \ldots \ldots \ldots \ldots \ldots$. $\ldots \ldots$ Page

8.1 INPUT TO NEPNERVA $\ldots \ldots \ldots \ldots \ldots \ldots \ldots \ldots \ldots \ldots$ Page 8-1

8.2 OUTPUT FROM NEPNERVA $\ldots \ldots \ldots \ldots \ldots \ldots \ldots \ldots$ Page 8.4

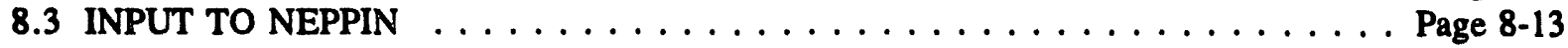

8.4 OUTPUT FROM NEPPIN $\ldots \ldots \ldots \ldots \ldots \ldots \ldots \ldots$ Page 8-15 
9.0 References $\ldots \ldots \ldots \ldots \ldots \ldots \ldots \ldots \ldots \ldots \ldots \ldots$. . . . . . . . . . . . . . .

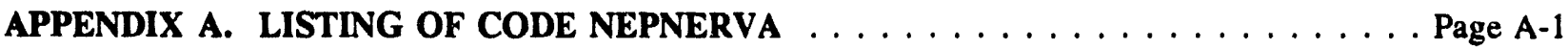

APPENDIX B. LISTING OF CODE NEPPIN $\ldots \ldots \ldots \ldots \ldots \ldots \ldots \ldots \ldots$ Page B-1 


\section{LIST OF TABLES}

Table 1. Reactivity worth of $\mathbf{B}_{4} \mathrm{C}$ sheets $\ldots \ldots \ldots \ldots \ldots \ldots \ldots \ldots$ Page 4 -2

Table 2. Reactivity worth (\%) of $25 \mathrm{~cm} \mathrm{BeO} \mathrm{reflector} \mathrm{for} \mathrm{the} \mathrm{fuel} \mathrm{pin} \mathrm{core} \mathrm{.} \mathrm{.} \mathrm{.} \mathrm{.} \mathrm{.} \mathrm{.} \mathrm{.} \mathrm{.} \mathrm{Page} \mathrm{4-10}$

Table 3. Reactivity worth $(\%)$ of $1 \mathrm{~cm}$ thick astar alloy pressure vessel $\ldots \ldots \ldots \ldots$. . . Page 4-16

Table 4. Reactivity worth (\%) of $360^{\circ}, 2 \mathrm{~cm}$ thick, $90 \%$ enriched $\mathrm{B}_{4} \mathrm{C}$ sheets $\ldots \ldots \ldots$ Page $4-16$

Table 5. Power and flow conditions $\ldots \ldots \ldots \ldots \ldots \ldots \ldots \ldots \ldots$ Page 6-1 


\section{LIST OF FIGURES}

Fig. 1. ${ }^{235} \mathrm{U}$ critical mass of the core and mass of the core plus reflector for the NERVA derivative reactor. $\ldots \ldots \ldots \ldots \ldots \ldots \ldots$ Page $4-3$

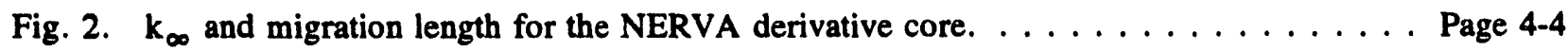

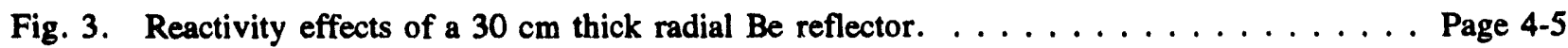

Fig. 4. Worth of the Be reflector (relative to $30 \mathrm{~cm}$ thickness) as a function of thickness. . . Page 4-6

Fig. 5. Reactivity worth of burnup for the NERVA derivative reactor, excluding Xe and Sm. . Page 4-7

Fig. 6. Fission distributions and absorption over fission ratio as a function of $\mathrm{S} / \mathrm{F} \ldots \ldots \ldots$. . . Page 4-8

Fig. 7. Reactivity worth of a $360^{\circ}, 2 \mathrm{~mm}$ thick, natural $\mathrm{B}_{4} \mathrm{C}$ located between the core and reflector as a function of $\mathbf{S} / \mathbf{F}$, also radial peaking factors are shown. . . . . . . . . . Page 4-9

Fig. 8. Fuel pin reactor, $\mathbf{k}_{\infty}$ as a function of pitch and enrichment. . . . . . . Page 4-11

Fig. 9. Fuel pin reactor, critical buckling $\left(\mathrm{cm}^{-1}\right)$ as a function of pitch and enrichment. . . . Page 4-12

Fig. 10. Fuel pin reactor, critical masses for spherical bare cores. . . . . . . . . . . Page 4-13

Fig. 11. Fuel pin reactor, spectras averaged in the core for reflected spheres. . . . . . . . Page 4-14

Fig. 12. Available energy in Mwd for the NERVA derivative reactor as a function of S/F . . . Page 7-4

Fig. 13. Available energy in Mwd for the fuel pin reactor as a function of enrichment $\ldots \ldots$ Page $7-5$

Fig. 14. Scheme for the exploration of possible designs, $E a$ is the available energy and $x$ is one of the core dimensions $\left(\mathrm{R}_{\mathrm{c}}\right.$ or $\left.\mathrm{H}_{\mathrm{c}}\right) \ldots \ldots \ldots \ldots \ldots \ldots \ldots \ldots \ldots \ldots \ldots$ Page $7.6 \ldots$ 


\section{ACKNOWLEDGEMENTS}

This work is the consequence of a collaborative agreement with the National Aeronautic and Space Administration (NASA), Lewis Research Center, and the Oak Ridge National Laboratory (ORNL). ORNL counterparts were B. A. Worley of the Engineering Physics and Mathematics Division (EPMD), R. Cooper and R. Holcomb of the Engineering Technology Division (ETD), and NASA counterparts were M. Doherty and J. Gilland from Lewis Research Center, Cleveland, Ohio. W. C. Jordan and J. C. Turner of the Computer Applications Division calculated the database for the fuel pin reactor design. 


\begin{abstract}
This technical memorandum describes models and calculational procedures used to fully characterize the nuclear island of power sources for nuclear electric propulsion. Two computer codes were written: one for the gas cooled NERVA derivative reactor and the other for liquid metal cooled fuel pin reactors. These codes are going to be interfaced by NASA with the balance of plant in order to make scoping calculations for mission analysis.
\end{abstract}




\subsection{FOREWORD}

Systems engineering efforts initiated by NASA's Lewis Research Center (LeRC) in FY92 under RTOP 593-72, for Nuclear Electric Propulsion (NEP), have enabled the development of detailed mathematical (computer) models to predict NEP subsystem performance and mass. The computer models are intended to help provide greater depth to NEP subsystem (and system) modeling, required for more accurately verifying performance projections and assessing the impact of specific technology developments.

The following subsystem models have been developed:

(1) Liquid-metal-cooled pin-type, and

(2) Gas-cooled NERVA (Nuclear Engine for Rocket Vehicle Applications) - derived for reactor/shield;

(3) Potassium-Rankine, and

(4) Brayton for power conversion;

(5) Heat rejection general model (includes direct Brayton, pumped loop Brayton, and shear flow condenser (Potassium-Rankine);

(6) Power management and distribution (PMAD) general model; and

(7) Magnetoplasmadynamic thruster for the electric propulsion subsystem.

These subsystem models for NEP were authored by the Oak Ridge National Laboratory (ORNL) for the reactor (NASA CR-191133, by the Rocketdyne Division of Rockwell International for Potassium Rankine (NASA CR-191134) and Brayton (NASA CR-191135) power conversion, heat rejection (NASA CR191132), and power management and distribution (NASA CR-191137).

At the time of this writing, these eight VAX/FORTRAN source and executable codes are resident on one of LeRC's Scientific VAX computers. 


\subsection{SUMMARY}

The modeling of a whole nuclear electric propulsion system for cargo and piloted missions is presently being developed at NASA Lewis Research Center. The vehicles would use either a Brayton direct conversion cycle using the heated helium from a NERVA type reactor or a potassium Rankine cycle with the working fluid heated at the secondary site of a heat exchanger with lithium at the primary site coming from a fast reactor.

This report describes the two computer codes written to fully characterize, for each of the options, the nuclear islands of the system. Given a set of input conditions, the codes calculate composition, dimensions, volumes, and masses of the core, reflector, control system, pressure vessel, neutron and gamma shields as well as the thermalhydraulic conditions of the coolant, clad and fuel. Input conditions are power, core life, burnup, pressure, and temperature of the coolant at the inlet of the core, and either the temperature of the coolant at the outlet of the core or the coolant mass flow.

Using state-of-the-art neutron cross sections and transport codes, a database was created for the neutronic performance of both reactor designs. The free parameters of the models are the moderating/fuel element ratio for the NERVA reactor and the enrichment and the pitch of the lattice for the fast reactor. Reactivity and energy balance equations are simultaneously solved to find the reactor design. Thermalhydraulic conditions are calculated by solving the one-dimensional versions of the equations of conservation of mass, energy, and momentum with compressible flow. The additional input conditions for the fluences and integrated doses at a cargo area of radius $\mathbf{r}$ located at distance $\mathbf{z}$ determines the dimensions, volumes, and masses of the neutron and gamma shields. The assumption was made to use either $\mathrm{LiH}$ in a stainless steel matrix or $\mathrm{B}_{4} \mathrm{C}$ for the neutron shielding and tungsten for the gamma shielding. 


\subsection{INTRODUCTION}

As a consequence of a collaborative agreement between NASA and ORNL, the Engineering Physics and Mathematics Division has been involved in the development of models and calculational procedures for the analysis (neutronic and thermalhydraulic) of power sources for nuclear electric propulsion.

The boundaries of the system to be modeled are the inlet and outlet plenums and the design variables of interest are the compositions, dimensions, volumes, and masses of the core, reflector, control system, pressure vessel, and neutron and gamma shields. The thermalhydraulic conditions of the coolant are also determined. Main input variables are power, core life, burnup, pressure, and temperature of the inlet coolant and its temperature at the outlet.

The computer codes developed are going to be coupled with the calculations of the balance of plant and ion generation and acceleration to perform scoping calculations for mission analysis. It was required then to develop codes that are fast running on PCs or workstations. For that reason, although the requested parameters are quite comprehensive, the models were kept as simple as possible.

The range of input conditions specified for this task were: a power range of 16 to $50 \mathrm{Mw}$ (th); operating lifetimes of 2 to 10 years; and outlet coolant temperature ranging from 1200 to $2200^{\circ} \mathrm{K}$.

\subsection{TYPES OF REACTORS CONSIDERED}

Two types of reactors were chosen for this analysis: the high temperature gas-cooled reactor of the NERVA derivative type and the lithium-cooled advanced fuel pin reactor, referred to as NERVA derivative and fuel pin for short.

The elements of the core of a NERVA derivative reactor are: (a) fuel elements with ZrC cladding, a dispersion of $\mathrm{UC}-\mathrm{ZrC}$ in a graphite matrix, and 19 coolant holes (diameter $2.88 \mathrm{~mm}$ ); and (b) support elements with $\mathrm{ZrH}_{2}$ on an inconel tube with pyrolitic graphite and graphite as a thermal shield. Both elements are hexagonal with $1.913-\mathrm{cm}$ flat-to-flat distance; by changing the ratio (S/F) of support to fuel elements the neutron spectrum can be modified considerably; the core is cooled by pumping He, which drives a turbine in a direct Brayton cycle. The reflector is made of beryllium, which contains the control drums that rotate the control boron carbide $\left(\mathrm{B}_{4} \mathrm{C}\right)$ sheets. The pressure vessel is located at the perip? of the reflector.

The core of the fuel pin reactor consists of bundles of rods containing UN pellets. The coolant, liquid lithium, removes heat from the core to boil potassium at the secondary side of a heat exchanger which drives a turbine using a Rankine cycle. The fuel rods have $6.4 \mathrm{~mm}$ outside diameter (O.D.) with 0.635 mm-thick cladding of the tantalum alloy Astar. A tungsten liner, 0.122-mm-thick, lays between the clad and the UN pellet, $4.786 \mathrm{~mm}$ in diameter with a 0.025 -mm-thick He gap surrounding the fuel pellet. The reflector is made of $\mathrm{BeO}$ which contains the control drums with the $\mathrm{B}_{4} \mathrm{C}$ absorber. The pressure vessel is located between the core and the reflector.

Common to both reactors are the material of the reflector pressure vessel (Astar alloy), the neutron shielding materials (lithium hydride $(\mathrm{LiH})$ in a stainless steel matrix or $\mathrm{B}_{4} \mathrm{C}$ ), and the gamma shielding material (tungsten). 


\subsection{NEUTRONIC CALCULATIONS}

In order to find the volume of the core, a reactivity balance is performed where the reactivity effects of the reflector, burnup, pressure vessel, structural materials, and desired reactivity at end of life (EOL) are added to the multiplication constant of the bare core. It was then necessary to create a data base for all these parameters; this section describes the criterion and results. Both reactors are intended to be controlled with absorbing materials in the reflector. Thus, the excess reactivity is produced by the reflector with the bare core near critical state. Other factors like compact designs and low masses favor cylindrical shapes with the core diameter $\left(2 R_{c}\right)$ approximately the same as the core height $\left(H_{c}\right)$ (i.e., $R_{c} / H_{c}=0.541$, this is the ideal shape from a neutron economy standpoint). Many of the data were then generated for a critical core of ideal shape with the transport code XSDRNPM ${ }^{1}$ using a very detailed cross section library. ${ }^{2}$

\subsection{NERVA DERIVATIVE DATA BASE}

The two free parameters of this design are the concentration of the fuel (highly enriched, $93 \%,{ }^{235} \mathrm{U}$ ) in the fuel element and the number of support-to-fuel-elements ratio. A plot of the critical mass of ${ }^{235} \mathrm{U}$ and the mass of the core plus the $24-\mathrm{cm}$ reflector is presented in Fig. 1 showing that beyond $\sim 500 \mathrm{~g}$ of ${ }^{235} \mathrm{U}$ per liter of fuel element the total mass is not substantially reduced (note that Fig. 1 is for an ideal shape, for odd shapes the situation might be different). It was decided to fix the fuel concentration to $500 \mathrm{~g}$ of ${ }^{235} \mathrm{U} / \mathrm{L}$ fuel; the database depends then on $\mathrm{S} / \mathrm{F}$ alone.

\subsubsection{Neutronic Properties of the Core}

Multiplication constants, $k_{\infty}$, for the infinite lattice and migration lengths, $M$, are plotted in Fig. 2 as a function of $S / F$.

\subsubsection{Reactivity Worth of Beryllium Reflector}

$\because$ The reactivity effects of a $30 \mathrm{~cm}$ thick radial reflector are shown in Fig. 3 as a function of S/F. It is defined as $\Delta k \equiv$ ( $k$ (reflected) $-k$ (bare) $) \times 100$, where $k$ 's are the multiplication constants. The effects of the thickness of the Be reflector are shown in Fig. 4. This shape of the curve is used for all S/F although Fig. 4 corresponds to $S / F=0.195$ and a fuel deissity of $374.7 \mathrm{~g}$ of ${ }^{235} \mathrm{U} / \mathrm{L}$ of fuel.

\subsubsection{Reactivity Worth of Burn-Up}

The reactivity worths of the burnup, expressed as a percentage of the ${ }^{235} \mathrm{U}$ loading at beginning of life (BOL), are shown in Fig. - The values shown were calculated by depleting the number densities of ${ }^{235} \mathrm{U}$ by the percentage burnup and simulating the fission products as a lumped $1 / \mathrm{v}$ absorber calibrated at 50 barn per fission at a energy of $0.025 \mathrm{eV}$.

Because the reactor can have a thermal spectrum because of the presence of support elements, the effects of Xe and Sm at stzady state and after transients were computed with formulas taken from Ref. 3. Effective cross sections for these formulas are obtained by collapsing a four-group cross section set and spectras used by the program; in this way, transition from thermal (where Xe poisoning is important) to epithermal spectras (where Xe is irrelevant) can be made gradually. Note that because of the way we computed its effects, $10 \%$ burnup means $10 \%$ depletion via fission and capture.

\subsubsection{Depletion of ${ }^{235} \mathrm{U}$}

Figure 6 illustrates how the fission distributions change as a function of S/F. The change of spectra affects the values of the absorption to fission ratio by as much as $10 \%$. This functional dependence is taken into account by the program. 


\subsubsection{Reactivity Worth of $\mathrm{B}_{4} \mathrm{C}$ Sheet}

The reactivity worth of $360^{\circ}$ sheets of $3_{4} \mathrm{C}$ located between the core and the reflector are shown in Table 1, which was prepared for a core with $S / F=0$ where the neutron spectra is the hardest. The maximum value would correspond to the removal of the reflector. Table 1 shows that a $2 \mathrm{~mm}$ thick sheet of natural $\mathrm{B}_{4} \mathrm{C}$ is very effective, so it was decided to use it as control material. The reactivity worths of $360^{\circ}, 2 \mathrm{~mm}$ thick natural $\mathrm{B}_{4} \mathrm{C}$ sheets are shown in Fig. 7 as a function of $\mathrm{S} / \mathrm{F}$ and for a $30 \mathrm{~cm}$ thick reflector; in general, as the moderation in the core is increased the reflector and anything within it is less important, including the control drums. The worth of the $\mathrm{B}_{4} \mathrm{C}$ is then multiplied by the factor given by Fig. 4 to compensate for effects of reflector thickness. Figure 7 also shows the peaking factor in the radial direction of the power density at BOL, i.e., with fresh fuel and control drums in.

Tsible 1. Reactivity worth of $\mathrm{B}_{4} \mathrm{C}$ sheets*

\begin{tabular}{|c|c|c|}
\hline Sheet & $\Delta k(\%)$ & $(\Delta k / \Delta k) \max$ \\
\hline $2 \mathrm{~mm} \mathrm{Nat} \mathrm{B}$ & 21.0 & 0.70 \\
\hline $2 \mathrm{~mm} \mathrm{80 \%} \mathrm{B}$ & $2 \dot{0} 0$ & $0.8 \mathrm{U}$ \\
\hline $5 \mathrm{~mm} \mathrm{90 \%} \mathrm{B}$ & 25.9 & 0.86 \\
\hline
\end{tabular}

*Core: $\mathrm{S} / \mathrm{F}=0, \mathrm{R}_{\mathrm{c}}=59.25 \mathrm{~cm}, \mathrm{H}_{\mathrm{c}}=109.11$. Reflector: $30 \mathrm{~cm} \mathrm{Be}(\Delta \mathrm{k}) \max =30.14 \%$ (removing the Be reflector). 


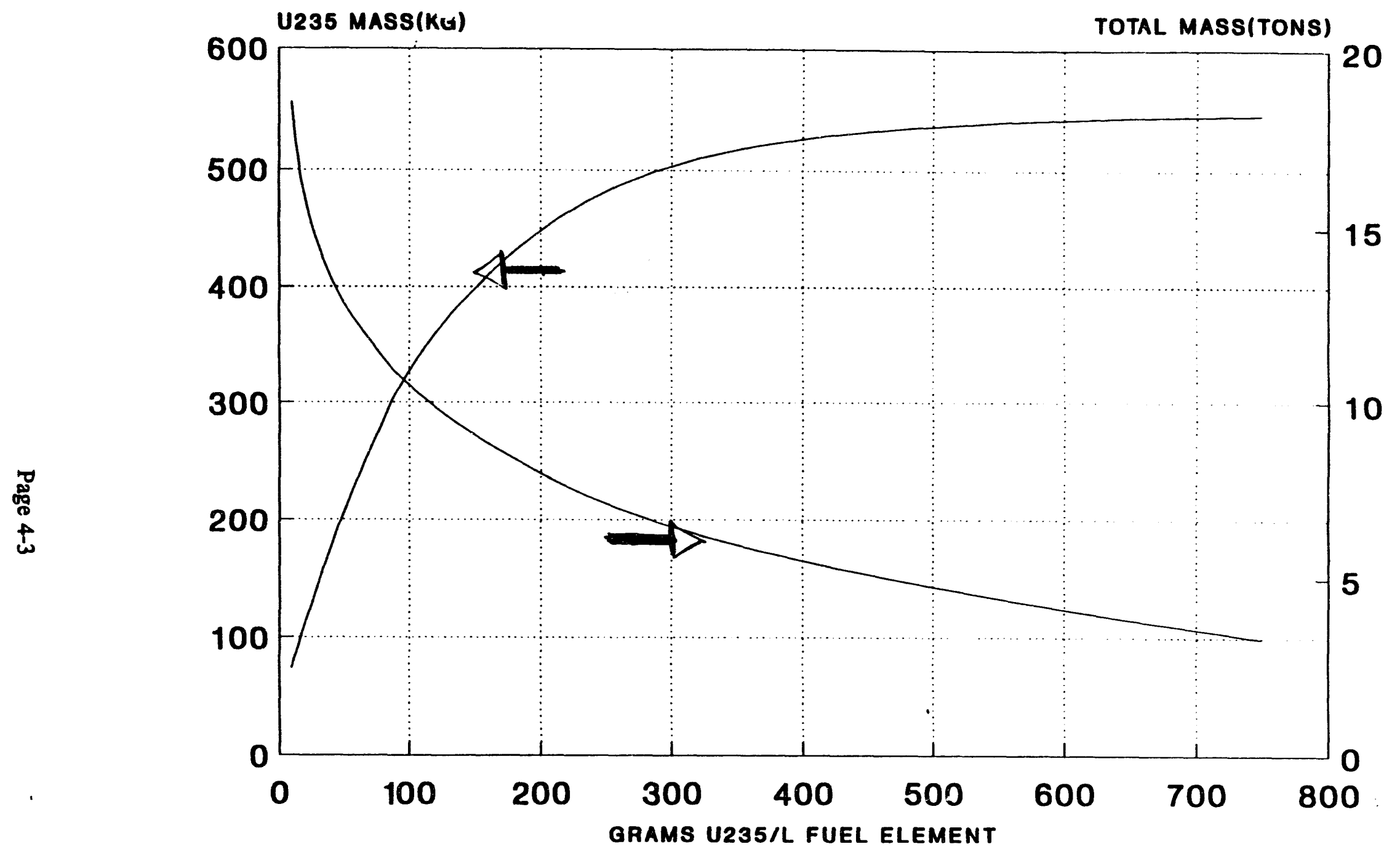

SEP 92; TOTAL MASS INCLUDES 24 CM BE REF

Fig. 1. ${ }^{235} \mathrm{U}$ critical mass of the core and mass of the core plus reflector for the NERVA derivative reactor. 


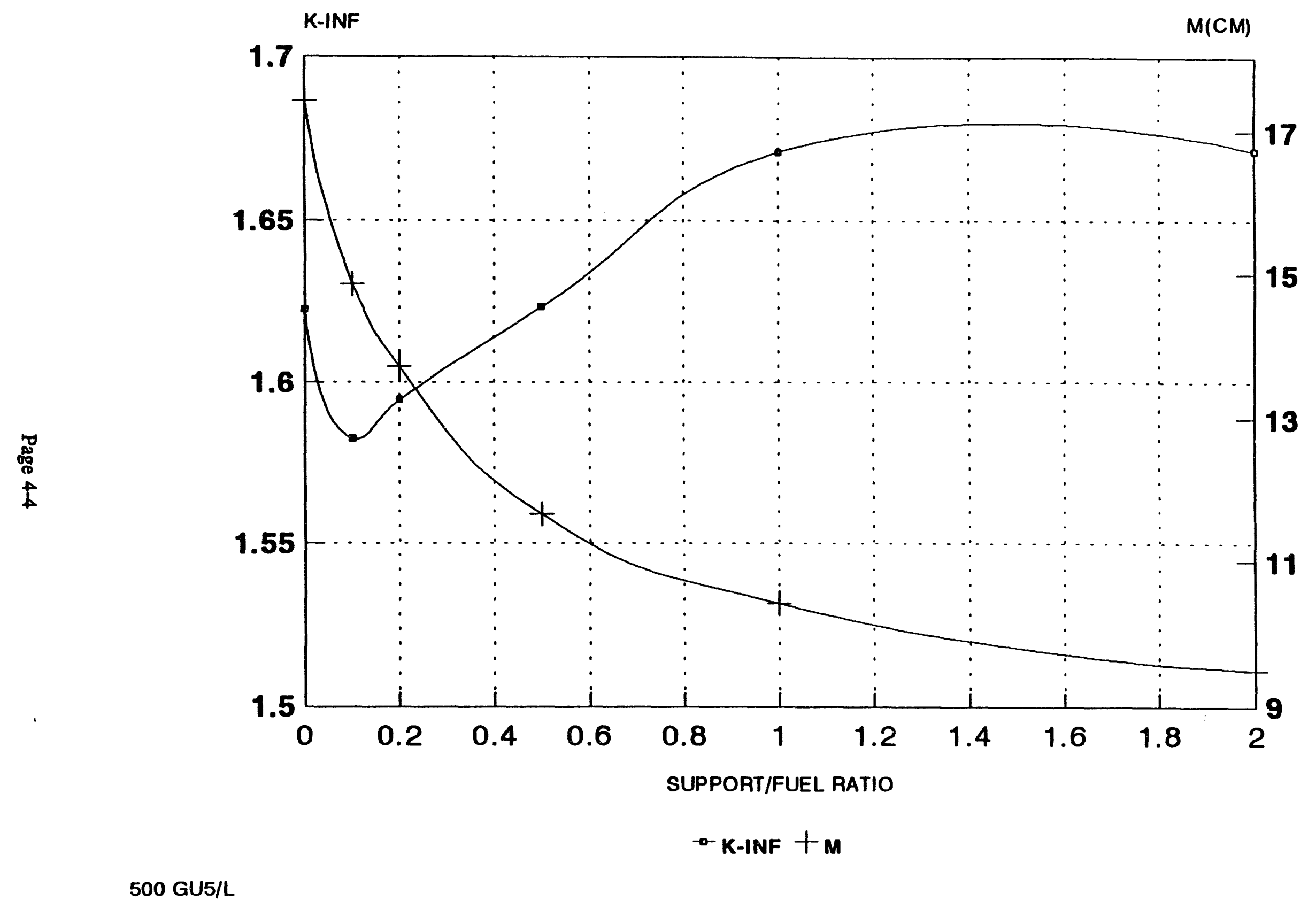

Fig. 2. $k_{\infty}$ and migration length for the NERVA derivative core. 


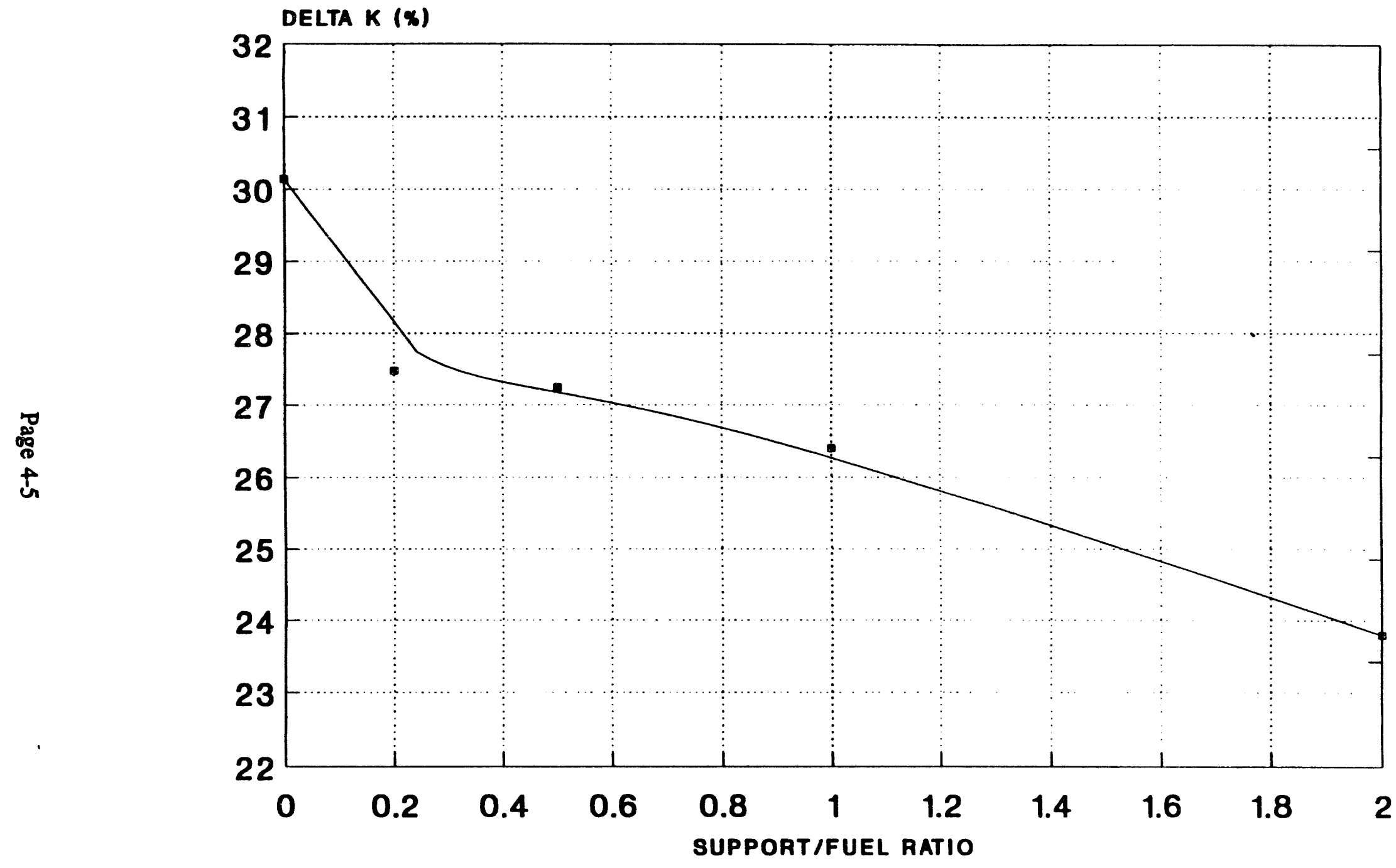

9-92

Fig. 3. Reactivity effects of a $30 \mathrm{~cm}$ thick radial Be reflector. 


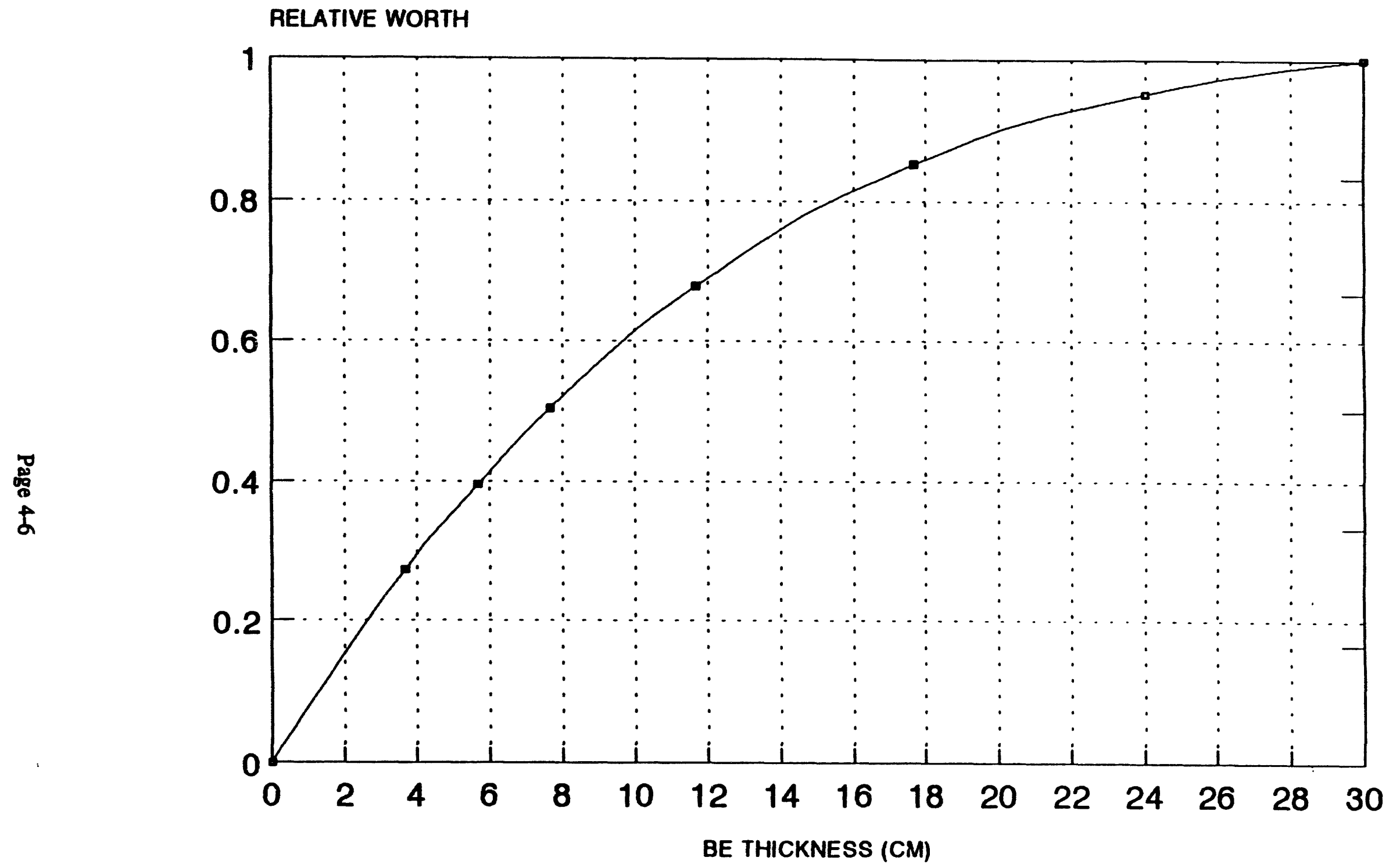

9-92; SUPP/FUEL ELEM $=0.195 ; \mathrm{RCORE}=48.33 \mathrm{CM}$

Fig. 4. Worth of the Be reflector (relative to $30 \mathrm{~cm}$ thickness) as a function of thickness. 


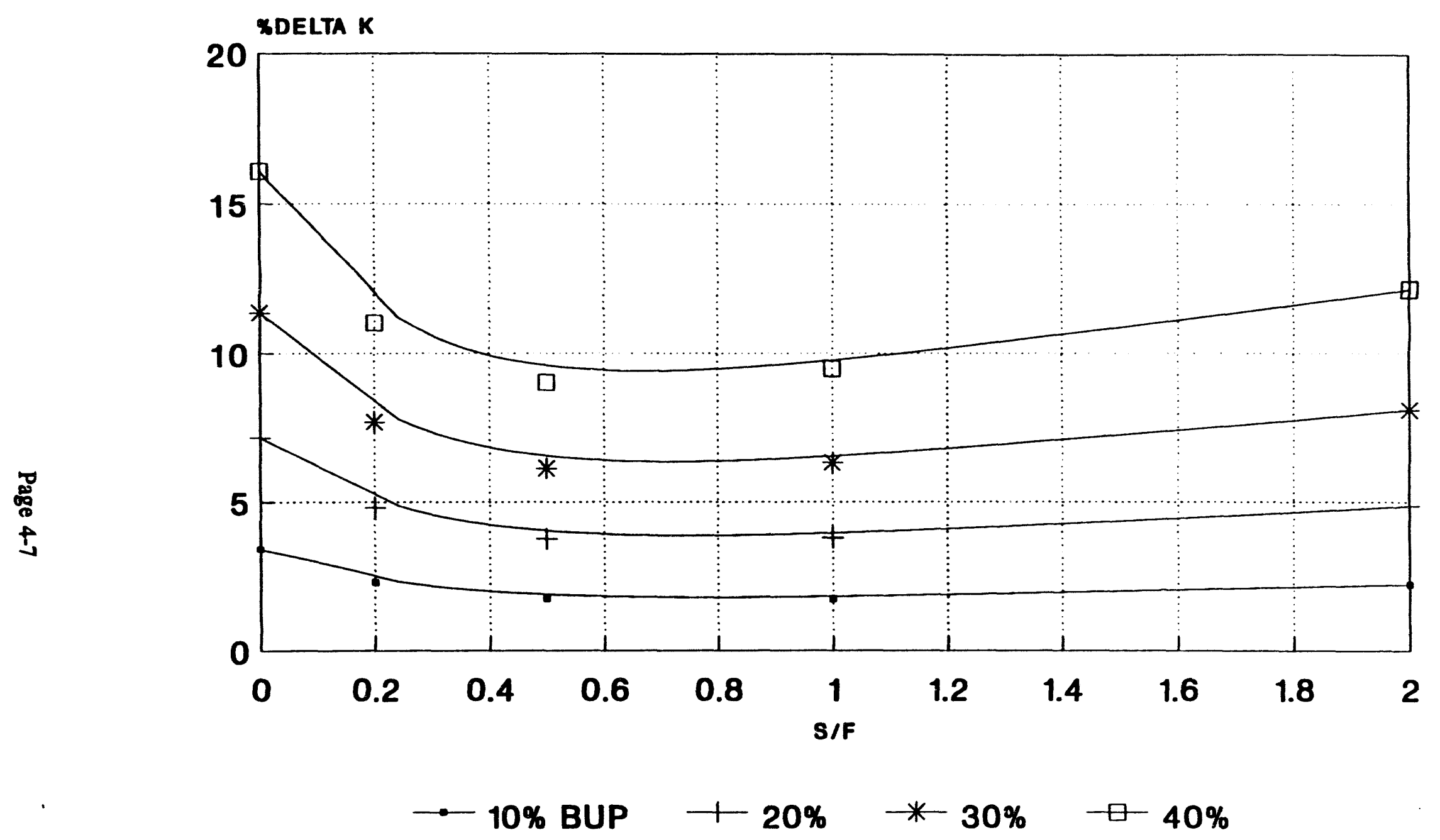

SEP 92; 500 G U235/L FUEL

Fig. 5. Reactivity worth of burnup for the NERVA derivative reactor, excluding Xe and Sm. 


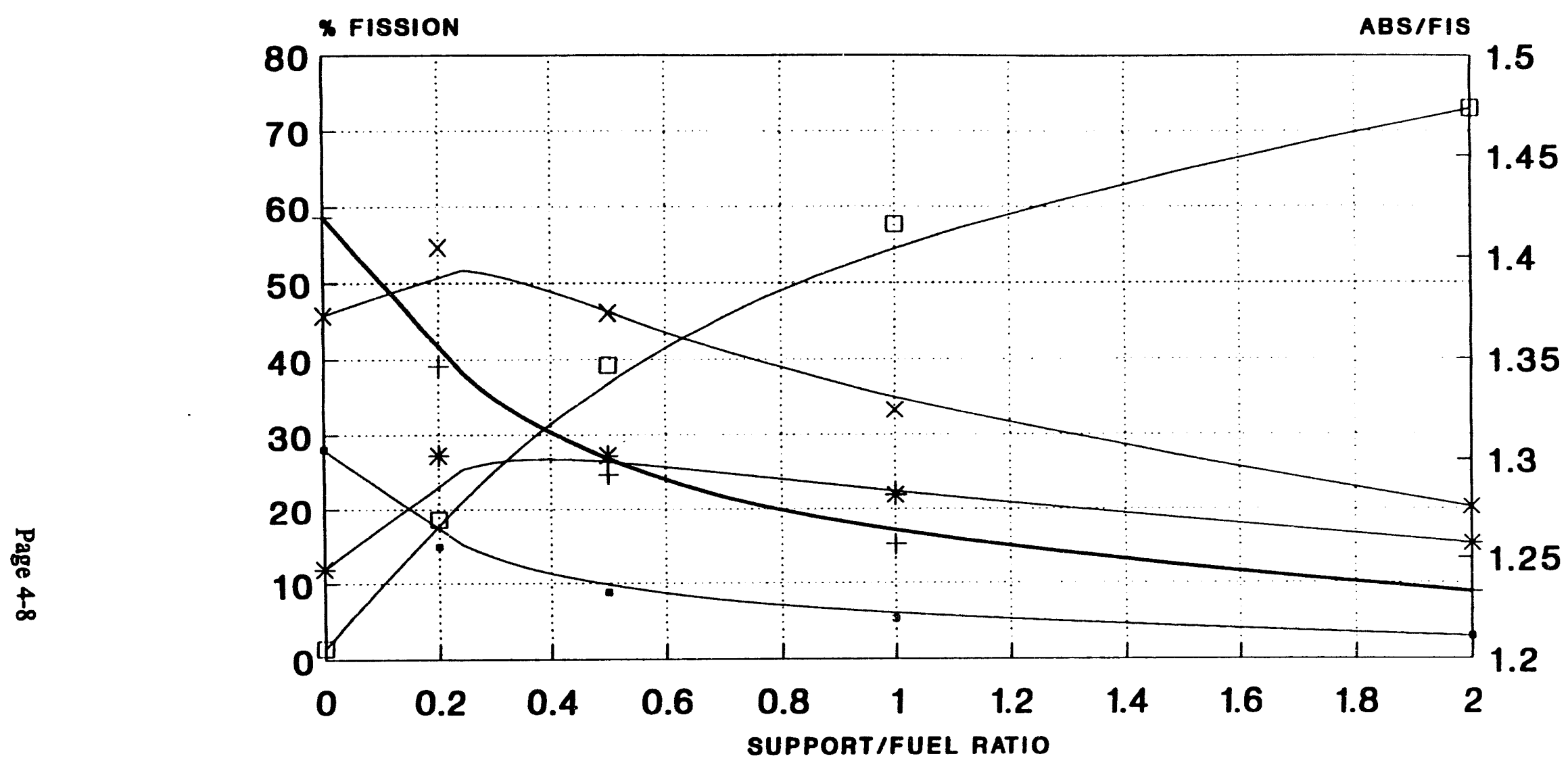

$\rightarrow \%$ FIS E’100. KEV + \% FIS E .1/100. KEV* \% FIS E $3 / 100$ EV 口 \% FIS E\&3 EV $\quad \times$ ABS/FISS

500 GU5/L;30.CM BE; $2 M M$ B4C

Fig. 6. Fission distributions and absorption over fission ratio as a function of S/F. 


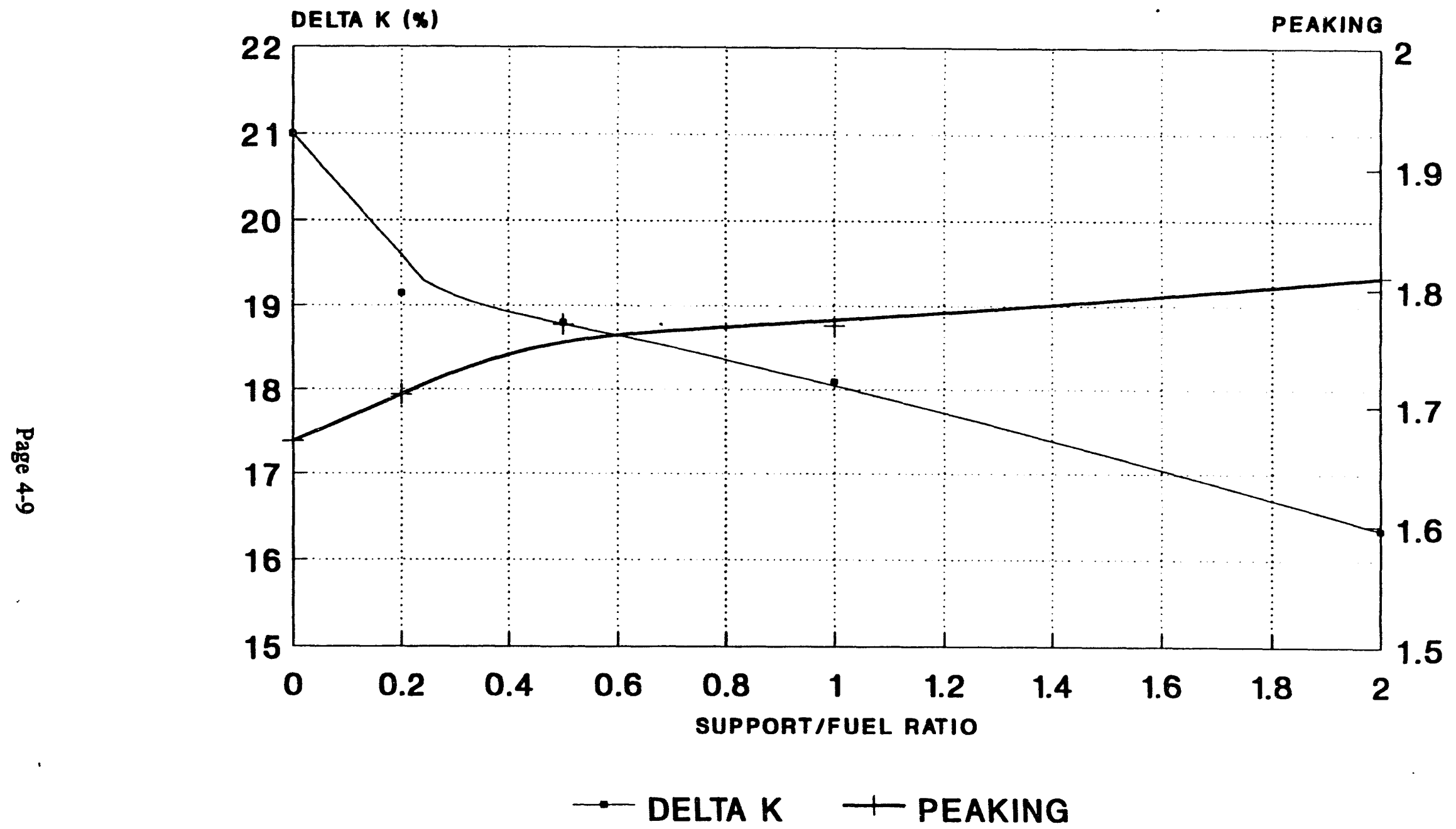

$500 \mathrm{GUS} / \mathrm{L}_{;} 30 . \mathrm{CM} B \mathrm{BE}_{\mathbf{2}} 2 \mathrm{MM} \mathrm{B} 4 \mathrm{C}$

Fig. 7. Reactivity worth of a $360^{\circ}, 2 \mathrm{~mm}$ thick, natural $\mathrm{B}_{4} \mathrm{C}$ located between the core and retlector as a function of $\mathrm{S} / \mathrm{F}$, also radial peaking factors are shown. 


\subsection{FUEL PIN DATABASE}

The two free parameters of this design are the enrichment and the pitch of the hexagonal lattice; a possible third one, the diameter of the fuel rod, was fixed to $6.4 \mathrm{~mm}$.

\subsubsection{Neutronic Properties of the Core}

Figures 8 and 9 show $k_{\infty}$ and critical bucklings as function of enrichment and pitch to diameter ratio, $\mathrm{P} / \mathrm{d}$. The corresponding critical masses for spherical bare reactors are shown in Fig. 10 . The comparison of this figure with Fig. 1 shows that a considerably larger amount of fuel can be assembled with the fuel pin option. Figure 11 shows spectras averaged in the core for reflected spheres.

\subsubsection{Reactivity Worth of Beryllium Oxide Reflector}

The reactivity worth of a $25 \mathrm{~cm}$ thick BeO reflector is shown in Table 2 as function of enrichments and P/d. The worths were calculated as the difference between the multiplication constant of radially reflected and bare critical cylindrical cores with optimum shape. For a different reflector thickness the worth of the reflector is multiplied by the factor of Fig. 4 due to the incomplete database.

Table 2. Reactivity worth (\%) of $25 \mathrm{~cm} \mathrm{BeO} \mathrm{reflector} \mathrm{for} \mathrm{the} \mathrm{fuel} \mathrm{pin} \mathrm{core}$

\begin{tabular}{|c|c|c|c|c|c|}
\hline \multirow{2}{*}{ P/d } & \multicolumn{5}{|c|}{ Enrichment (\%) } \\
\cline { 2 - 6 } & 93.0 & 80.0 & 70.0 & 60.0 & 50.0 \\
\hline 1.1 & 19.26 & 17.26 & 15.28 & 12.78 & 9.57 \\
\hline 1.2 & 18.57 & 16.30 & 14.11 & 11.40 & 8.04 \\
\hline 1.3 & 17.65 & 15.18 & 12.85 & 10.02 & 6.58 \\
\hline 1.4 & 16.61 & 13.99 & 11.55 & 8.65 & 5.22 \\
\hline 1.5 & 15.50 & 12.76 & 10.25 & 7.32 & 3.95 \\
\hline
\end{tabular}

\subsubsection{Reactivity Worth of Burnup and Isotopics}

Because of the lack of a database for this effect a simplified analytical approach was chosen. Assuming that the leakage does not change with burnup, the change in the multiplication constant because of the burnup (BU) is:

$$
\Delta k(B U) \equiv k(B U)-k(0)=\frac{k_{\infty}(B U)}{k_{\infty}(0)}-1
$$




\section{NASA Parametrics}

K-infinity vs P/D

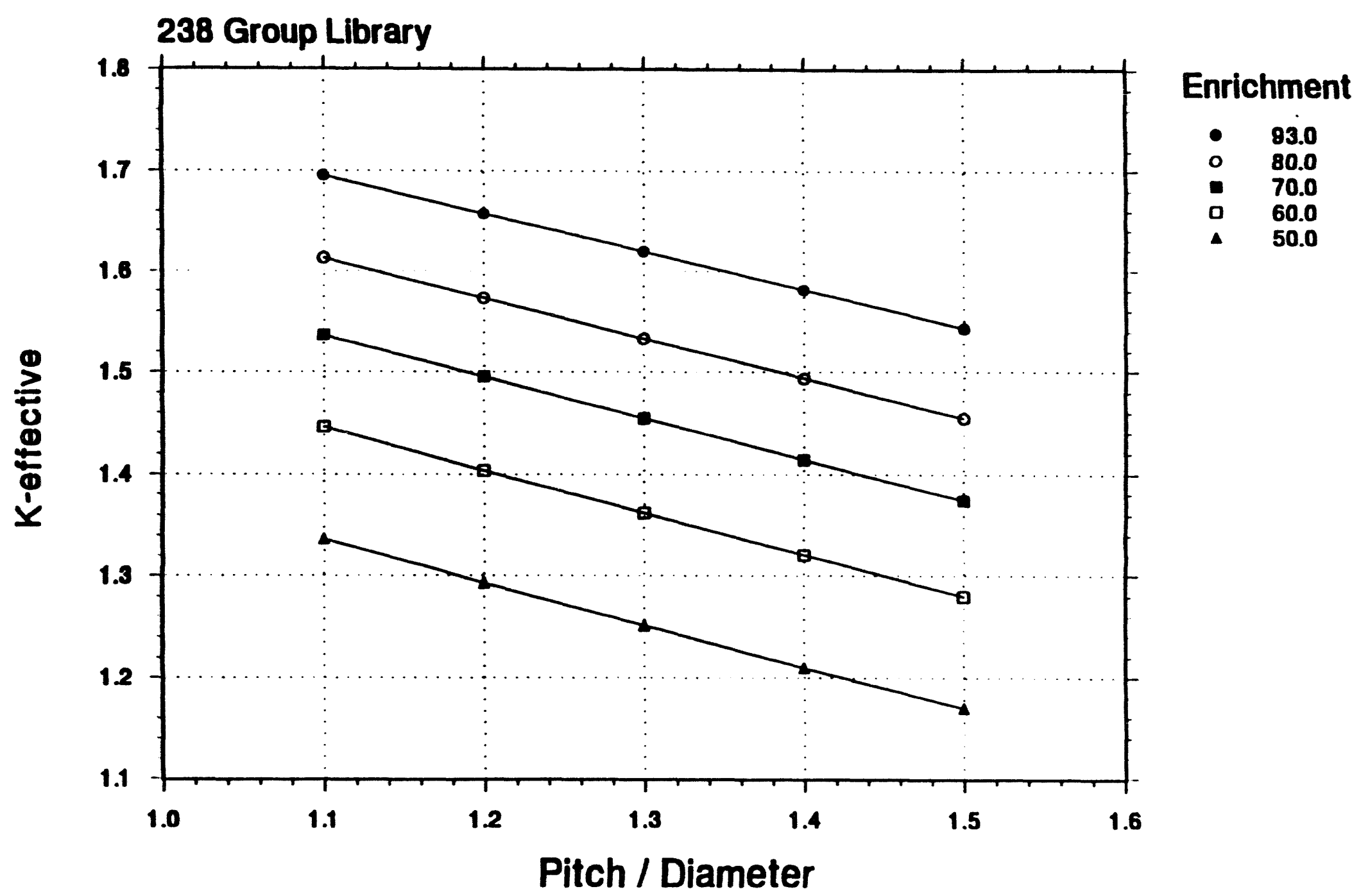

Fig. 8. Fuel pin reactor, $k_{\infty}$ as a function of pitch and enrichment. 
NASA Parametrics

B3 Buckling Calc. vs P/D

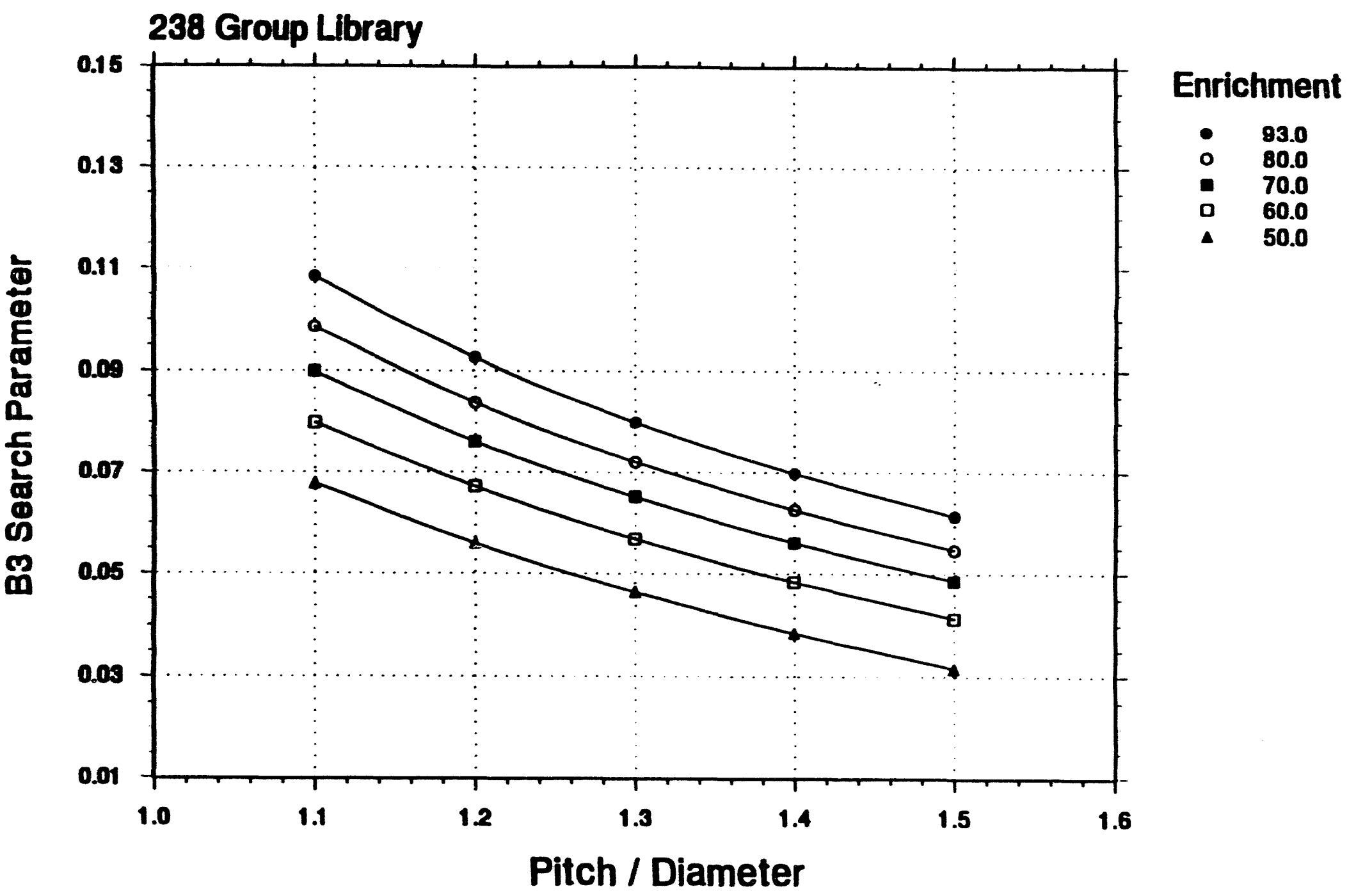

Fig. 9. Fuel pin reactor, critical buckling $\left(\mathrm{cm}^{-1}\right)$ as a function of pitch and enrichment. 


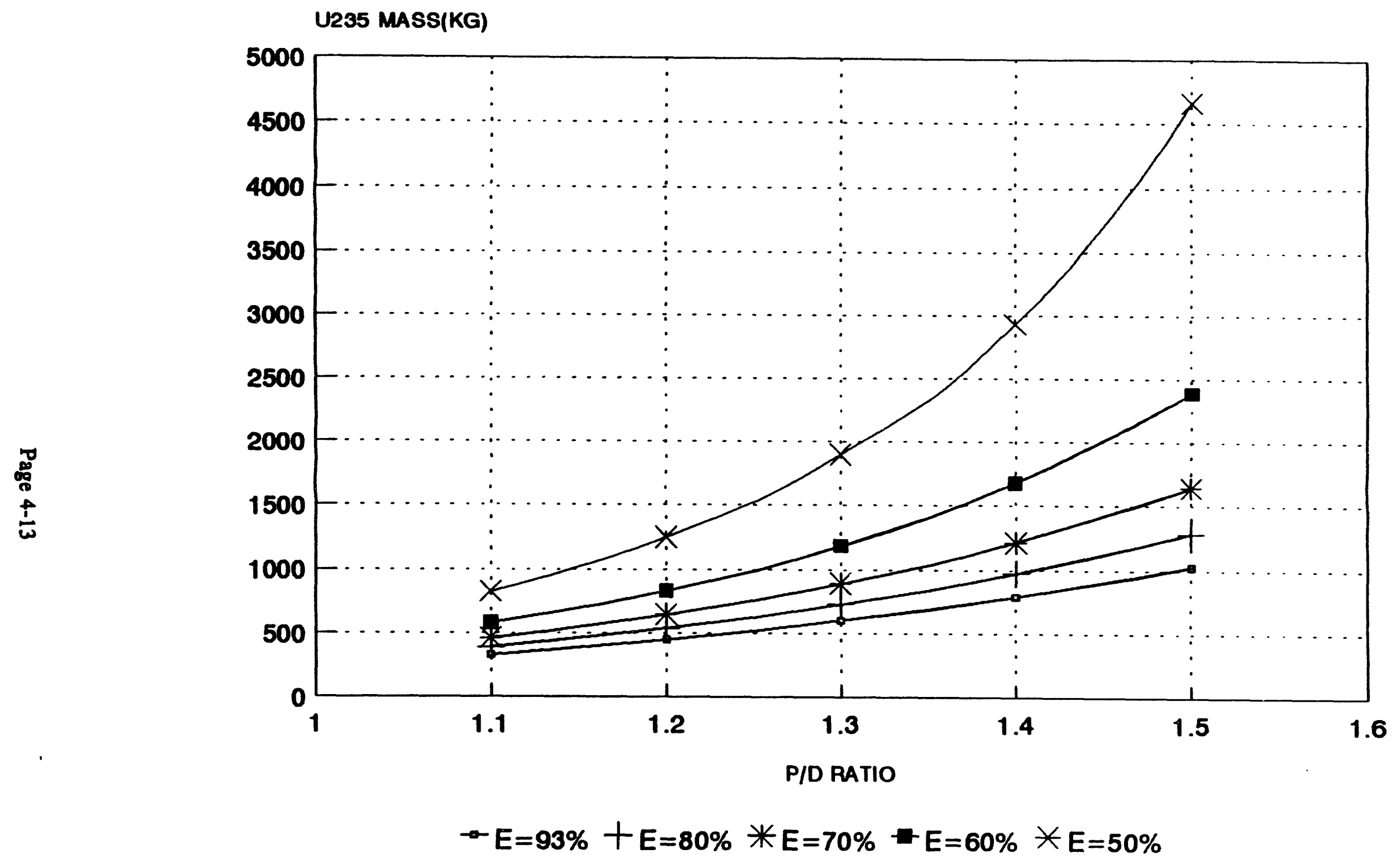

1-93;0.64 CM FUEL RODS

Fig. 10. Fuel pin reactor, critical masses for spherical bare cores. 


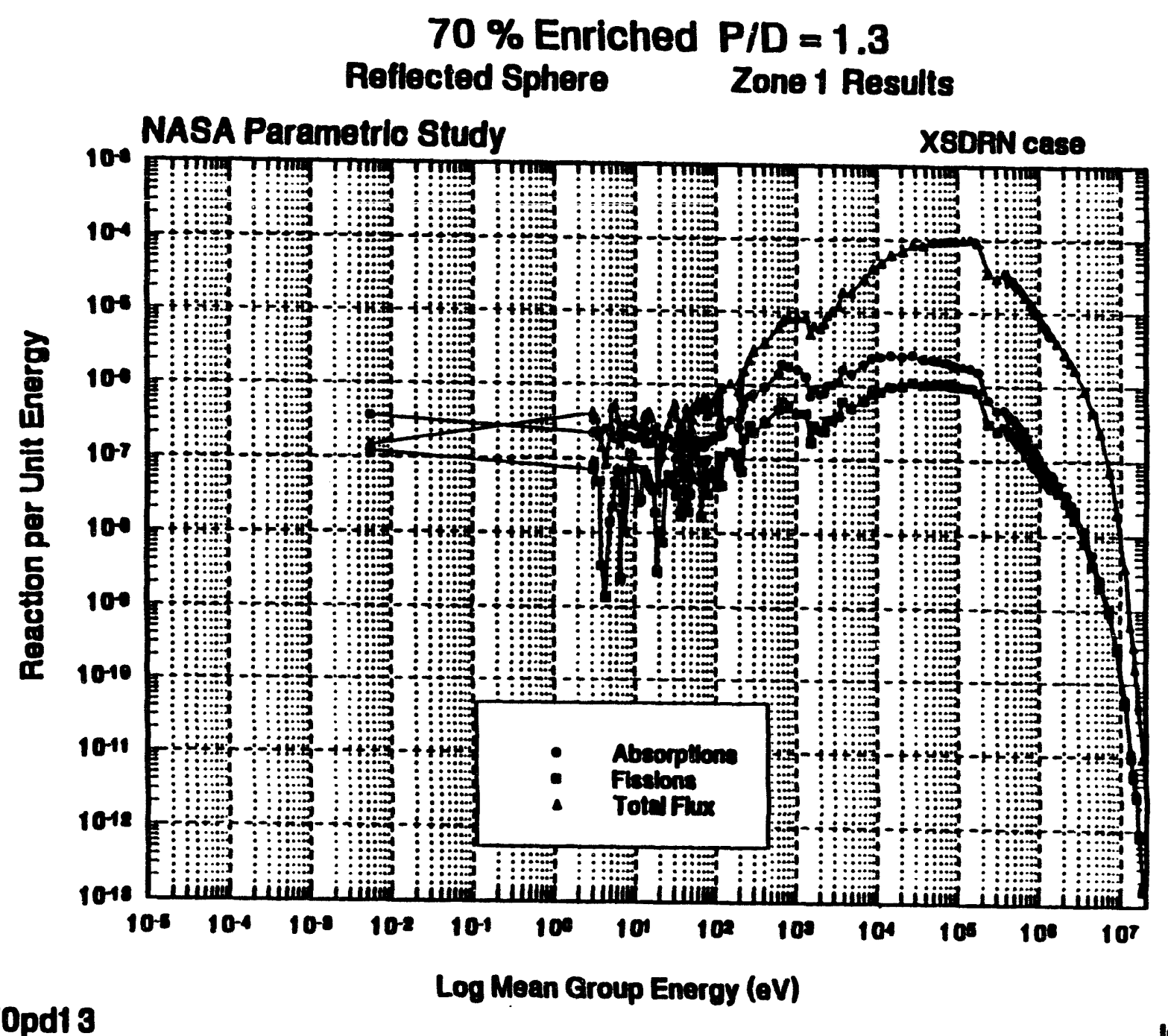

e70pd1 3

JCT 1/07/93

Fig. 11. Fuel pin reactor, spectras averaged in the core for reflected spheres. 
where the multiplication constant for the infinite lattice is

$$
k_{-}=\frac{v_{s} n_{5} \sigma_{f g}+v_{8} n_{8} \sigma_{f 8}+v_{9} n_{9} \sigma_{f g}}{n_{5} \sigma_{a s}+n_{8} \sigma_{a 8}+n_{9} \sigma_{a q}+n_{f p} \sigma_{a, f p}+\Sigma_{r}}
$$

where indexes 5, 8, and 9 stand for ${ }^{235} \mathrm{U},{ }^{238} \mathrm{U}$, and ${ }^{239} \mathrm{Pu}, f p$ for fission products, and $r$ for the rest of the mixture. The $\sigma$ 's stand for cross sections averaged in the neutron spectra and $n_{5}, n_{8}, n_{9}$, and $n_{f p}$ are the isotopic concentrations which are functions of the burnup. The burnup is defined as the integrated number of fissions relative to the initial concentration of ${ }^{235} \mathrm{U}$ atoms,

$$
B U \equiv \frac{f_{3}+f_{8}+f_{9}}{n_{5}(0)}
$$

Note, that because of the way we compute the burnup this definition refers to depletion via fission only and is slightly different with respect to the case of the NERVA reactor.

Writing and solving the buildup and depletion equations for the isotopic and assuming that there is no $\mathrm{Pu}$ at BOL, we obtain

$$
\begin{aligned}
& B U=\frac{\sigma_{f s}}{\sigma_{a 8}}\left(1-e^{-\sigma_{a b \phi 1}}\right)+\frac{\sigma_{f 8}}{\sigma_{a 8}}\left(\frac{1}{\varepsilon}-1\right)\left(1-e^{-\sigma_{a b \phi 1}}\right) \\
& +\frac{\sigma_{a 8}}{\sigma_{a q}-\sigma_{a 8}}\left(\frac{1}{\varepsilon}-1\right)\left[\frac{\sigma_{f g}}{\sigma_{a 8}}\left(1-e^{-\sigma_{a b \phi}}\right)-\frac{\sigma_{f g}}{\sigma_{a 8}}\left(1-e^{-\sigma_{a q \phi \ell}}\right)\right]
\end{aligned}
$$

where indices $f, a$, and $c$ stand for fission, absorption and capture, $\varepsilon$ is the initial enrichment, and the $\sigma$ 's were taken from Ref. 4. For an input burnup, BU, Eq. 4 is solved for the fluence $\phi t$ which is then used to find the isotopic concentrations necessary to evaluate Eqs. (1) and (2).

\subsubsection{Reactivity Worth of the Pressure Vessel}

Because in this design the pressure vessel is located between the core and the reflector it is relevant to the reactivity balance. Table 3 shows the reactivity worths as a function of enrichment and $\mathrm{P} / \mathrm{d}$. 
Table 3. Reactivity worth (\%) of $1 \mathrm{~cm}$ thick astar alloy pressure vessel*

\begin{tabular}{|c|c|c|c|c|c||}
\hline \multirow{2}{*}{ P/d } & \multicolumn{5}{|c|}{ Enrichment (\%) } \\
\cline { 2 - 7 } & 93.0 & 80.0 & 70.0 & 60.0 & 50.0 \\
\hline 1.1 & 5.69 & 5.43 & 5.10 & 4.57 & 3.73 \\
\hline 1.2 & 5.39 & 5.06 & 4.63 & 3.99 & 3.05 \\
\hline 1.3 & 5.12 & 4.66 & 4.14 & 3.44 & 2.45 \\
\hline 1.4 & 4.77 & 4.23 & 3.67 & 2.92 & 1.91 \\
\hline 1.5 & 4.41 & 3.82 & 3.22 & 2.44 & 1.43 \\
\hline
\end{tabular}

*Worth is negative, $25 \mathrm{~cm} \mathrm{BeO} \mathrm{reflector,} \mathrm{critical} \mathrm{bare} \mathrm{cylindrical} \mathrm{core} \mathrm{with} \mathrm{optimum} \mathrm{shape.}$

The worth of the pressure vessel is multiplied by the factor given by Fig. 4 to consider the effects of a different reflector thickness.

\subsubsection{Reactivity worth of $\mathrm{B}_{4} \mathrm{C}$ sheets}

The reactivity worths of $360^{\circ} 2 \mathrm{~cm}$ thick, $90 \%$ enriched $\mathrm{B}_{4} \mathrm{C}$ sheets located between the pressure vessel and the $\mathrm{BeO}$ reflector are shown in Table 4.

Table 4. Reactivity worth (\%) of $360^{\circ}, 2 \mathrm{~cm}$ thick, $90 \%$ enriched $\mathrm{B}_{4} \mathrm{C}$ sheets*

\begin{tabular}{|c|c|c|c|c|c||}
\hline \multirow{2}{*}{ P/d } & \multicolumn{5}{|c|}{ Enrichment (\%) } \\
\cline { 2 - 6 } & 93.0 & 80.0 & 70.0 & 60.0 & 50.0 \\
\hline 1.1 & 6.73 & 5.98 & 5.23 & 4.29 & 3.12 \\
\hline 1.2 & 6.64 & 5.74 & 4.92 & 3.91 & 2.69 \\
\hline 1.3 & 6.37 & 5.43 & 4.55 & 3.50 & 2.24 \\
\hline 1.4 & 6.07 & 5.08 & 4.15 & 3.06 & 1.81 \\
\hline 1.5 & 5.73 & 4.69 & 3.73 & 2.63 & 1.39 \\
\hline
\end{tabular}

*Worth is negative, $25 \mathrm{~cm} \mathrm{BeO}$ reflector, critical bare cylindrical core with optimum shape and a $1 \mathrm{~cm}$ thick astar alloy pressure vessel.

The table shows low effectiveness of the $\mathrm{B}_{4} \mathrm{C}$ sheet because of the shielding effects of the $1 \mathrm{~cm}$ thick pressure vessel. Better location for the sheet might be found, but no additional data is available. Corrections to the database because of different thicknesses for the pressure vessel and the $\mathrm{B}_{4} \mathrm{C}$ sheets are discussed in Section 6. 


\subsection{SHIELdNG CALCULATIONS}

The materials for the shielding are $\mathrm{LiH}$ in a stainless steel matrix, or $\mathrm{B}_{4} \mathrm{C}$, for the neutrons, and tungsten for the gammas, in a shadow shield configuration. The input parameters for the designs are accepted values for fluences and integrated gamma doses for the entire duration of the mission at a cargo area located at $z$ meters from the base of the reactor and with radial size $r$.

After its evaluation, the source term for each radiation is affected by the self shielding of the reactor materials. Removal cross sections and buildup factors are used to calculate the thickness of the shield and the radial sizes are chosen by assuming the shield plate is at the base of the reactor and that there is no line of sight from any point of the reactor to the cargo area. Both shielding requirements were calculated together because one type of shielding material shields, marginally, the other type of radiation.

\subsection{NEUTRON SHELDING}

For an input power, the source term of fast neutrons is calculated assuming a value of $200 \mathrm{Mev}$ per fission. The self shielding, $f_{s}$, for an uncollided current of fast neutrons in spherical geometry, and uniform composition within radius $R$, is

$$
f_{z}=\frac{3}{a}\left\{\frac{1}{2}-\frac{1}{a^{2}}\left[1-e^{-a}(1+a)\right]\right\},
$$

where $a=2 \Sigma R$ and $\Sigma$ is the removal cross section. We had used an equivalent $R$ for the total volume of the core, reflector and pressure vessel and a uniform density of materials with removal cross section from Ref. 5. Given the source term, the self shielding $f_{3}$, the accepted fluence, and the removal cross sections of the $\mathrm{LiH}$-ss or $\mathrm{B}_{4} \mathrm{C}$, the thickness of the neutron shield is fixed.

\subsection{GAMMA SHILLDING}

The source term is calculated from the total number of fissions and captures, both determined by the power level. The capture reaction rate is then distributed into their material components with relative capture rates read from tables (case of NERVA derivative) or calculated with one-group capture cross sections (the case of the fuel pin). The next step is to multiply capture and fissions rates with the 9 group gamma production spectra per atom taken from Ref. 5.

The self shielding factor is given also by Eq. (5) but with the final value attenuated by the buildup factor due to the scattering of gamma rays. Removal cross sections and buildup factor parameters are also from Ref. 5. The definition of buildup factors for a mixture, even homogeneous, is not obvious; we have interpolated the tables with an equivalent atomic number for the mixture defined with the $\mathbf{Z}$ of the components weighted with their total gamma reaction rates. Given the source terms, the self shielding $f_{z}$ and the accepted gamma dose, the thickness of the tungsten plate is determined after reading cross sections and buildup factors from the data base for the 9 energy groups. 


\subsection{THERMALHYDRAULICS}

Thermalhydraulic conditions are calculated with the approximation of one-dimensional equations for the conservation of mass, energy and momentum. Three steps are involved in the calculations related with the calculations of the bulk conditions of the coolant, the temperature of the wall of the channel and the temperatures within the fuel.

Thermalhydraulic variables depend on power and flow conditions. Four are considered in this analysis and are described in Table 5.

Table 5. Power and flow conditions

\begin{tabular}{|c|c|c|c|c|}
\hline \multirow[b]{2}{*}{ Case } & \multicolumn{2}{|c|}{ Power distribution } & \multirow{2}{*}{$\frac{\text { Flow distribution }}{r, \theta}$} & \multirow[b]{2}{*}{ Comments } \\
\hline & $\mathbf{r}, \theta$ & $\mathbf{z}$ & & \\
\hline 1 & Uniform & Uniform & Uniform & 3-D fuel grading \\
\hline 2 & Uniform & Cosine & Uniform & 2-D $(r, \theta)$ fuel grading \\
\hline 3 & Not uniform & Cosine & $\begin{array}{l}\text { Proportional to } \\
\text { local power }\end{array}$ & $\begin{array}{l}\text { Uniform fuel, flow } \\
\text { conditioning }\end{array}$ \\
\hline 4 & Not uniform & Cosine & Uniform & Uniform fuel \\
\hline
\end{tabular}

Case 1 is the most relaxed and case 4 the most extreme; intermediate cases 2 and 3 give very similar results. Please note that fuel grading, cases 1 and 2 , implies reactivity penalties not considered in the balance of reactivity in Section 3.

Power density, released as heat, at the location of the fissile material is

$$
\rho_{P}=\bar{\rho}_{P} \frac{f_{1}}{f_{2}} F(r, \theta, z)
$$

where $\bar{\rho}_{p}=\mathrm{P} / \pi R_{c}^{2} H_{c}$ is the fission power density averaged into core of radius $R_{c}$ and height $H_{c}, f_{1}$ is the fraction of the fission power released as heat inside the core, $f_{2}$ is the volumetric fraction of the fissile material relative to the core volume and $F(r, \theta, z)$ is the power distribution of the homogenized core,

$$
F(r, \theta, z)=\frac{\pi R_{c}^{2} H_{c} \psi(r, \theta, z)}{\int_{\text {core }} \Psi(r, \theta, z) d V}
$$


We assume the distribution $\boldsymbol{\Psi}$ can be written as

$$
\Psi(r, \theta, z)=R(r, \theta) Z(z)
$$

Consequently

$$
F(r, \theta, z)=f_{z} f_{r, \theta}
$$

where

$$
f_{z}=\frac{Z(z)}{\frac{1}{H_{c}} \int_{0}^{H_{c}} Z(z) d z}
$$

and

$$
f_{r, \theta}=\frac{R(r, \theta)}{\frac{1}{\pi R_{c}^{2}} \int_{0}^{R_{c}} r d r \int_{0}^{2 \pi} R(r, \theta) d \theta} .
$$

We further assume that $Z(z)$ can be approximated as

$$
Z(z)=\sin \frac{\pi z}{H_{c}^{\prime}},
$$

where $H_{c}^{\prime}=H_{c}+28$, with $\delta$ being the extrapolated length and the fuel occupying the space between $z=\delta$ and $z=H_{c}+\delta$. Eq (10) can then be approximated as

$$
f_{z}=\frac{\alpha}{\sin \alpha} \sin \frac{\pi z}{H_{c}^{\prime}}
$$

where $\alpha=\pi H_{c} /\left(2 H_{c}^{\prime}\right)$. 
Because of the even distribution of control drums around the reflector and because the assumed even movement of the absorbing sheets, $R(r, \theta)$ is a periodic function in $\theta$ with period $\theta_{d}=2 \pi / N_{d}$ where $N_{d}$ is the number of drums, $R(r, \theta)$ can then be expanded in Fourier series, resulting in

$$
f_{r, \theta}=a_{0}(r)+\dot{\sum}_{n=1}\left[a_{n}(r) \cos n 2 \pi \frac{\theta}{\theta_{d}}+b_{n}(r) \sin n 2 \pi \frac{\theta}{\theta_{d}}\right],
$$

where

$$
\begin{aligned}
a_{n} & =\frac{\frac{1}{\pi R_{c}^{2}} \int_{0}^{R_{c}} \bar{R}_{0}(r) 2 \pi r d r}{\bar{R}_{n}(r)} \\
b_{n} & =\frac{\frac{1}{\pi R_{c}^{2}} \int_{0}^{R_{b}} \bar{R}_{0}(r) 2 \pi r d r}{\bar{R}_{n}^{\prime}(r)} \\
\bar{R}_{n}(r) & =\frac{1}{\theta_{d}} \int_{0}^{\theta_{d}} R(r, \theta) \cos \left(n 2 \pi \frac{\theta}{\theta_{d}}\right) d \theta
\end{aligned}
$$

and

$$
\bar{R}_{n}^{\prime}(r)=\frac{1}{\theta_{d}} \int_{0}^{\theta_{d}} R(r, \theta) \sin \left(n 2 \pi \frac{\theta}{\theta_{d}}\right) d \theta
$$

At BOL and with the absorbing sheets looking to the core, we assume that $f_{r, 0}$ is maximum at the center of the core, where $a_{n}=b_{n}=0$ for all $n \neq 0$, i.e.,

$$
f_{r, 0} l_{\max }=a_{0}(r=0)
$$

and that $f_{r, 0}$ is minimum at $r=R$ and in front of the absorbing sheet

$$
\left.f_{r, 0}\right]_{\min }=a_{0}\left(r=R_{c}\right)-\Delta_{\theta}
$$


where $-\Delta_{0}$ is the sum of Eq. (14) evaluated at $r=R_{c}$ and the $\theta$ corresponding to the minimum, or

$$
\left.f_{n, 0}\right]_{\min }=a_{0}\left(R_{c}\right)\left[1-\frac{\Delta_{0}}{a_{0}\left(R_{c}\right)}\right]
$$

We had tabulated $a_{0}(r)$ by using the approximation of considering a continuous $360^{\circ}$ sheet of absorber rather than a collection of discrete strips around the core. Also the angular tilt in the bracket of Eq. (21) was left as an input variable in the programming. In this way, and for cases 3 and 4 of Table 5, the program computes the thermalhydraulic conditions for the two extreme channels.

\section{6.i BULK CONDITIONS OF COOLANT}

For the case of the bulk conditions of the coolant and considering no gravitational effects and one phase flow, the equations of conservation are

$$
\begin{gathered}
\rho v=\rho_{1} v_{1} \\
h+\frac{v^{2}}{2}=h_{1}+\frac{v_{1}^{2}}{2}+\frac{P_{1}}{w}
\end{gathered}
$$

and

$$
w \frac{d v}{d z}+A \frac{d p}{d z}+\frac{1}{2} \rho v^{2} f P_{f}=0
$$

where the channel was subdivided into nodes, subindex $i$ refers to the inlet conditions and the unsubscripted variables refer to the outlet conditions. Variables $p, v, h$ and $p$ are density velocity, enthalpy and pressure of the fluid, $w$ is the constant mass flow through a constant area $A, P_{1}$ is the power delivered to the node, $f$ is the friction factor and $\boldsymbol{P}_{\boldsymbol{f}}$ the wet perimeter.

\subsubsection{Bulk Conditions of the Hellum Coolant}

Because of the compressible flow and the high speed of the coolant, Eqs. (22) to (24) have to be solved without further simplifications. Combining Eqs. (22) and (23) we obtain

$$
F(p, T)=h-h_{1}-\frac{P_{1}}{w}-\frac{v_{1}^{2}}{2}\left(1-\frac{p_{1}^{2}}{p^{2}}\right)=0 .
$$


Using the equation of state, Eq. (24) can be written as

$$
\frac{d p}{d z}\left[1-\frac{v^{2}}{c^{2}}\right]+\frac{w^{2}}{A^{2} p}\left[\frac{f P_{f}}{2 A}-\frac{1}{p} \frac{\partial p}{\partial T} \frac{d T}{d z}\right]=0
$$

where $c=1 /(\partial \rho / \partial p)_{T}$ is the isothermal speed of sound. Integrating Eq. (26) along the node we have

$$
G(p, T) \equiv p-p_{i}+\bar{F}_{1} \Delta z+\bar{F}_{2}\left(T-T_{i}\right)=0
$$

where $\bar{F}_{1}$ and $\bar{F}_{2}$ are the average values within the node of

$$
F_{1}=\frac{w^{2} f P_{f}}{2 A^{3} p} /\left(1-\frac{v^{2}}{c^{2}}\right)
$$

and

$$
F_{2}=\frac{w^{2}}{A^{2} \rho^{2}} \frac{\partial p}{\partial T} /\left(1-\frac{v^{2}}{c^{2}}\right)
$$

The averages are approximated by weighing the inlet and outlet conditions

$$
\bar{F}_{e} \cong \frac{1}{1+x}\left[F_{e}\left(p_{1}, T_{1}\right)+x F_{e}(p, T)\right], e=1,2
$$

where $x$ is the relative weight. The outlet conditions, $p$ and $T$, are obtained by the simultaneous solutions of Eqs. (25) and (27). Because Eq. (25) is mainly dependent on $T$, a first estimation of the temperature $T^{(1)}$ is made by using $p_{l}$ instead of $p$ in Eq. (25). With $T^{(1)}$, Eq. (27) is solved for $p$ to obtain a first estimation of $p, p^{(1)}$; then $F$ and $G$ are linearized around $p^{(1)}, T^{(1)}$ to find $p, T$. In the whole process $x$ is an input value.

When $v$ approaches sonic velocities the entire one dimensional approach is no longer valid. If this condition occurs, the program prints warnings and restarts calculations relaxing inlet and power conditions. 
Friction factors, thermodynamics, and transport properties of the He were taken from Ref. 6. For Reynolds, Re, number below 2,000, $\left(R e=4 w / \mu P_{f}, \mu\right.$ viscosity),

$$
f=\frac{16}{R e} \text {, }
$$

and above $R e=2000$ the Moody approximation to the Colebook-White correlation was used

$$
f=0.001375\left[1+\left(20,000 \cdot \frac{\varepsilon}{d}+\frac{10^{6}}{R e}\right)^{1 / 3}\right],
$$

which includes $\varepsilon$, the rugosity of the channel with diameter $d$.

\subsubsection{Bulk Conditions of the Liquid Lithium}

Considerable implications are possible in this case; because the dependence of the enthalpy with the pressure is negligible as well as the velocity terms, Eq. (25) is used to evaluate $T$ which then defines $p$ and the velocity. With the known velocity gradient and the average of the friction term in the node also known, Eq. (24) can be immediately integrated to find $p$.

The fuel rods are assembled in hexagonal bundles and they are separated with helicoidal spacing wires. One result of this arrangement is that there are three types of cells, central, lateral and at the corner with different flow fractional areas. The thermalhydraulics calculations are done for the 3 cells and the average conditions across the bundle are then calculated. For the calculation of the average pressure distribution in the bundle, we have used the Novendstern correlation taken from reference 7 which includes the effects of the spacing wire and the different geometries of the 3 cells within the bundle. The temperature of the hottest point in the coolant is monitored against the saturation temperature to warn the user about potential local boiling. Thermodynamics and transport properties of $\mathrm{Li}$ were taken from reference 8.

\subsection{WALL TEMPERATURE}

After the calculation of the bulk temperature of the coolant, the wall temperature, $T_{w}$, is calculated with the equation

$$
T_{w}=T_{b}+\frac{j}{h}
$$

where $\boldsymbol{j}$ is the power current and $\boldsymbol{h}$ is the heat transfer coefficient, obtained from correlation for the Nusselt number $N u=h D / k$. 


\subsubsection{Wall Temperature of the NERVA Derivative Fuel Element}

Although velocities of the He coolant are not very high for NEP, the program was prepared to deal with the case of high speed flow, like in the case of NTP. At very high speed, $T_{b}$ in Eq. (33) is substituted by $T_{b}^{\prime}$ winich is given by

$$
F_{R}=\frac{T_{b}^{\prime}-T_{b}}{T_{s}-T_{b}}
$$

where the recovery factor, $F_{R}=\sqrt[3]{\operatorname{Pr}}$ (Prandtl number, $\operatorname{Pr}=C_{p} \mu / k$ ) and the stagnation temperature, $T_{s}$, is given by

$$
h\left(T_{s}, p\right)=h\left(T_{b}, p\right)+v^{2} / 2
$$

The correlation of Mc Eligot et. al. ${ }^{6}$ was chosen for the Nusseit number. It is the well known DittusBoelter correlation with corrections because of entry effects and non-negligible differences between wall and bulk temperatures. The complications of all these corrections make the wall temperature an implicit variable so an iterative method is necessary.

\subsubsection{Wall Temperature of the Fuel Rods}

Because of the excellent heat conductivity of the liquid lithium and the higher density we do not have the complications of the previous case, namely aerodynamics and temperature gradient effects, so explicit calculations with Eq. (33) are possible and no iterations are necessary. The correlations for the Nusselt number in pages 189, 190 of Ref. 7 were used; the correlations depend mainly on the Peclet number, $\boldsymbol{P}_{e}=\operatorname{Re} \boldsymbol{P r}$, and are specially fitted for rods in a bundle. They are parametric in $P / d$ and in the effective ratio of the eddy diffusivity of heat to momentum, $\Psi$, which depends on Prandtl and Reynolds numbers and $P / d$.

\subsection{FROM WALL TO FUEL TEMPERATURES}

Once the wall temperature is known, the temperature distributions inside the solid fuel element are calculated by solving the one dimensional (this time the radial direction) heat conduction equation. The maximum fuel temperature is then compared with the melting temperature of the fuel to warn the user to relax the input requirements.

\subsubsection{Fuel Temperature of the NERVA Derivative Reactor}

The coolant flows through holes in the hexagonal fuel element; centered in the coolant hole and for increasing radius $r$ we find the wall at $r=r_{0}$, the fuel at $r=r_{1}$ and the boundary of the heat cell at $r=r_{2}$. Between $r_{0}$ and $r_{1}$ we have the $Z r C$ clad, and between $r_{1}$ and $r_{2}$ a mixture of graphite and $U C-Z r C$; the radius of the cell, $r_{2}$, is determined by distributing the area of the mixture evenly between the nineteen holes.

The temperature distribution equations are subject to the boundary conditions $T\left(r=r_{0}\right)=T_{w}$ (from Section 5.2) and $\partial T / \partial r)_{r=r_{2}}=0$. 
The heat conductivity $(k)$ of the different materials was taken from the available literature. If hetter values become available the subroutines could be reprogrammed easily. The $k$ of the cladding was taken from Ref. 10, $k(\operatorname{ZrC})=0.2077 \frac{\text { watk }}{\mathrm{cm} K}$; the $k$ of the mixture is calculated as

$$
k(m i x)=x(U C) k(U C)+x(Z r C) k(Z r C)+x(\text { graph }) k(\text { graph })
$$

where the $x$ 's are the mass fractions in the mixture. The temperature dependent $k(U C)$ values were taken from Ref. 9. The $k$ of graphite exhibits large anisotropic and irradiation effects; ${ }^{10}$ no intentions were made to introduce these additional complications so average temperature dependent $k$ 's without irradiation effects were used. Graphite melts at $3889^{\circ} \mathrm{K}$ above the melting temperature of the $U C-\mathrm{ZrC}$ mixture which depends on the relative concentration. Melting temperatures of the UC-ZrC mixture were taken from Ref. 10.

\subsubsection{Fuel Temperature of the Fuel Pin Reactor}

Centered in the fuel rod we have the $U N$ fuel pin to $r=r_{p}$, the He gap to $r=r_{\text {c }}$ and the tungsten liner and astar alloy clad up to $r=r_{r}$. Heat conductivities were taken from reference 4 and the heat transfer of the He gap was computed as $k(H e) /$ gap thickness, with $k(H e)$ from Ref. 6 . This value is a simplification; very detailed discussions about this parameters can be found in Ref. 7. Boundaries conditions are $T\left(r=r_{r}\right)=T_{w}$ (from Section 5.2.2) and $\left.\partial T / \partial r\right)_{r=0}=0$. Melting temperature of $U N$ and $W$ quoted in the program are from Ref. 11.

\subsection{THERMALHYDRAULIC FEDDBACK VIA THE PRESSURE VESSEL}

Strictly speaking the thermalhydraulic conditions have an effect over the neutronic calculations of Section 3. This feedback was not considered because it is a small effect in comparison with the scoping nature of the calculations and the uncertainties of the models. The exception is the pressure vessel for the case of the fuel pin reactor. The thickness $d$ of the pressure vessel is determined in general by the equation ${ }^{12}$

$$
d=\frac{p R}{0}
$$

where $R$ is the external radius of the Be reflector for the NERVA derivative reactor or the radius of the core for the fuel pin reactor, $p$ is the pressure, and $\sigma$ is the maximum stress allowable for the pressure vessel. We have used a subroutine from the ALKASYS ${ }^{4}$ code to calculate $\sigma$, determined by the temperature and the life of the reactor. The temperature of the pressure vessel is an input variable. If it is not specified, the code chooses the exit temperature of the coolant as the temperature of the pressure vessel.

Because the pressure vessel has an impact on the reactivity balance (see Table 3) its worth is included in the reactivity balance. 


\subsection{ORGANIZATION OF THE CODES}

In order to find a design that meets the input demands two balance equations have to be simultaneously solved; one for the reactivity, the other for the energy. The major input variables are the reflector thickness, $\delta_{\mathrm{g}}$, the burnup at EOL, BU, the power level, $P$, the operation time, $D$, and the boundary conditions for the thermalhydraulics: inlet conditions of the coolant, $\mathrm{p}_{\mathrm{i}}$ and $\mathrm{T}_{\mathrm{i}}$, and either its outlet temperature, $T_{0}$, or mass flow, $w$, and the temperature of the pressure vessel, $T_{p v}$.

The coolant outlet temperature or its flow is then calculated with an enthalpy balance equation neglecting the pressure drop along the reactor. If $T_{p v}$ is not specified the code uses $T_{0}$ as the temperature of the pressure veasel. $T_{p v}$ and $D$ are used then to compute $\sigma$, the maximum stress for the astar alloy.

\subsection{SELECTION AND CALCULATION OF A DESIGN}

In order to write the reactivity balance equation, the worth of each component is read from tables or computed. The reactivity worth of the radial reflector, $\Delta k_{(R)}$, is read from tables for a reference thickness and then modified by the factor of Fig. 4 because of the offects of input $\delta_{R}$. The worth of axial reflectors, if any, are computed in the same way with the additional factor of $1 / 2$, which is the ratio for axial over radial leakages for ideal shaped cylinders.

The reactivity worth of the burnup $\Delta k(B U)$ is also read from tables or computed, and the reactivity worth of the preasure veasel $\Delta k(P V)$ is parameterized in terms of o and the radius of core; additional terms are the desired reactivity at EOL, $\triangle k(E O L)$, and an estimation of the reactivity effects of structural parts $\Delta k(S P)$ which are assumed input variables.

The reactivity balance equation is then written as:

$$
k(B O L)=1+\Delta k(B O L)=k_{b}+\Delta k(R)-\Delta k(B U)-\Delta k(P V)-\Delta k(S P)
$$

where the multiplication constant of the bare core is:

$$
k_{b}=\frac{k_{\infty}}{1+M^{2} B^{2}}
$$

and the geometrical buckling is

$$
B^{2}=\left(\frac{\pi}{H_{C}}\right)^{2}+\left(\frac{2.405}{R_{c}}\right)^{2}
$$

Equations (38) and (39) are then solved for the size of the core by specifying either ideal shape $\left(R_{c} / H_{c}=0.54\right), R_{c}$ or $H_{c} ;$ in this process, some of the $\Delta k$ 's might be a function of the size so iterations are necossary (see next sections).

With $R_{c}$ and $H_{c}$ and the composition of the core, the total mass of fuel can then be calculated. Given this mass, the burnup and the scaling of $200 \mathrm{MeV}$ per fission, the calculation of the available energy, Ea, that the reactor can release during time $D$ is determined.

Figures 12 and 13 are examples of the available energy for both reactors. In order to match the requested energy $\mathrm{Er}=\mathrm{PD}$, the free parameters of each design are iterated until $\mathrm{Ea}=\mathrm{Er}$. If this cannot be done because of contradictions between the requests and what is possible, the codes relax, under options, the input conditions. 
The organization of the codes follows the shapes of Figs. 12 or 13. The scheme for the exploration of possible designs is shown in Fig. 14. For a given set of input conditions, the possible designs are within the region of the $\left(E_{2}, x\right)$ plane limited by parametric curves $p_{1}$ and $p_{n}$. For example, $p_{1}=S / F=2$, and $p_{n}=S / F=0$, for the NERVA reactors and $p_{1}=$ enrichment $=93 \%$ and $p_{n}=$ enr $=50 \%$ for the fuel pin reactors with $P / d=1.1$. The parametric curves have a minimum corresponding to the ideal shape, vertical asymptotic lines $A_{1}, A_{n}$ to the left and monotonic growth to the right. For example, if $x$ is the radius of the core, to the left of the minimum we have cigar shape designs while pancake shapes correspond to the region to the right.

For each parameter, the codes calculate the asymptotic lines and the energy available for the optimum shapes, $E_{0}$; under a test option these values are printed together with $E a$ as function of $R_{c}$ and $H_{c}$.

If the requested energy has a value, $\mathrm{Er}_{1}$, below the minimum of the $\mathrm{Eo}, \mathrm{E} \mathrm{o}_{\min }, \mathrm{i}$.e., if $\mathrm{Er}$ is in region 1 of Fig. 14, there is a warning message and the codes, under option, proceed to change the requested input burnup, lowering it until $\mathrm{EO}_{\min }$ is smaller than $\mathrm{Er}_{1}$ and choosing the ideal shape disregarding any input $\mathbf{R}_{\mathrm{c}}$ or $\mathbf{H}_{\mathrm{e}}$.

If the requested energy has a value, $\mathrm{Er}_{2}$, intermediate between the minimum and maximum of Eo, i.e., Er is in region 2 of Fig. 14, two things can happen: (1) if the ideal shape was chosen the codes proceed with their calculation, or (2) if the user chooses $\mathbf{R}_{c}$ or $\mathbf{H}_{c}$ then the codes compute the allowable ranges $x_{1}$ and $x_{2}$ in Fig. 14 which are then compared with the input dimension. If consistent, the codes proceed with the calculations; if not and under option the codes change the input dimension to $0.5\left(x_{1}+x_{2}\right)$.

If the requested energy has a value of $\mathrm{Er}_{3}$ within region 3 of Fig. 14, designs are only possible between $x_{1}$ and $x_{2}$ and between $x_{3}$ and $x_{4}$ and ideal shape designs are not feasible. The codes then compute $x_{1}$, $x_{2}, x_{3}$, and $x_{4}$ and switch the option for the shape to a core with dimension $x=1.01 x_{3}$ if allowed.

Assured now that the requested energy and shape are within curves $p_{1}$ and $p_{n}$ of Fig. 14, designs with parameters $p_{1}$ and $p_{n}$ bracket the demand 80 the codes iterate until a value $p_{r}$ is found that produces the required energy Er.

The calculations are then continued with the computation of the number densities and masses of the core, the reflector and the pressure vessel. For the case of the fuel pin reactor, the entire process is made for $P / d=1.1,1.2,1.3,1.4$, and 1.5; one design is then chosen either by the user or by the code which chooses the deaign with the lowest total mass from those that have a pressure drop below a prescribed input value. With a design chosen, the codes proceed to calculate the geometries and masses of the neutron and gamma shieldings and the thermalhydraulic conditions for the most and least heated channels.

\subsection{DESIGN OF THE CONTROL DRUMS}

The number of control drums is, in principle, an input variable, $n_{d}$, but it can be changed by the program. It is compared with the maximum number $n_{d(\max )}=\operatorname{Integer}(2 \pi / \Delta \theta)$ where $\Delta \theta=2$ arcain $\left(r_{d} /\left(R_{r}+r_{d}\right)\right.$ is the central parallax of the drums, $r_{d}$ is the radius of the drums (1/2 of the reflector thickness) and $R_{T}$ is the internal radius of the reflector; if larger the program continues with $n_{d}=n_{d\left(m_{a}\right)}$. The reactivity worth per unit angle is calculated as $w_{0}=\Delta k\left(B_{4} C\right) / 2 \pi$ where the numerator is read from tables as indicated in Sections 3.1.5 and 3.2.5; the maximum reactivity of the drums, $w_{0} \Delta \theta n_{(m a)}$, is then compared with the reactivity to control $\Delta k_{c}=k(B O L)-1$. $-\Delta k(S P)+\Delta k(S U B)$, where $\Delta k(S U B)$ is the desired subcriticality with the absorbers facing the core. If the maximum worth of the drums is smaller than $\Delta k_{c}$ a warning message is printed and the program bypasses what follows. 
The input number of drums is used to compute their maximum worth $\Delta k_{d}=w_{\theta} \Delta \theta n_{d}$ and if smaller than $\Delta k_{c}, n_{d}$ is increased to a value $n_{d}^{\prime}$ such that $\Delta k_{d}^{\prime}=w_{\theta} \Delta \theta n_{d}^{\prime}>\Delta k_{c}$. With $n_{d}$ now fixed the central parallax of the absorbing part of the drum is computed as $\Delta k_{c}=w_{\theta} \Delta \theta_{c} n_{d}$ and then converted to local parallax (centered in the drums).

\subsection{SOME DETAILS OF THE CODE NEPNERVA}

The burnup contributions to the reactivity Eq. (38) is split as the sum of three terms $\Delta k(X e), \Delta k(S m)$ and the $\Delta \boldsymbol{k}$ corresponding to ${ }^{235} \mathrm{U}$ depletion and other fission products as discussed in Section 3.1.3; this was done because as S/F increases the reactor becomes more moderated making necessary the special treatment for the large thermal neutron absorbers ${ }^{135} \mathrm{Xe}$ and ${ }^{149} \mathrm{Sm}$. The transition of the importance of these isotopes, from irrelevant at $S / F=0$ to very serious at $S / F=2$, is made smoothly with cross sections that depend on S/F.

Steady and transient $\mathrm{Xe}$ and transient $\mathrm{Sm}$ reactivity effects depends on the flux; because for a given power the flux depends on the volume, Eq. (38) becomes then implicit on the size of the core and it has to be iterated. Equations for steady and transient reactivity effects for Xe and Sm were taken from Ref. 3.

\subsection{SOME DETAILS OF THE CODE NEPPIN}

Because of the fast spectrum, the effects described in the previous sections do not appear in this case, instead an iteration because of the pressure vessel becomes necessary when solving the reactivity Eq. (38). The reactivity effects of the pressure vessel of thickness $d$, around a core of radius $R_{c}$ inside a reflector of thickness $\delta_{r}$, was parameterized as

$$
\Delta k(P V)=\Delta k_{n}(P V)\left(\frac{d}{d_{n}}\right)\left(\frac{R_{c n}}{R_{c}}\right) f\left(\frac{\delta_{r}}{\delta_{m}}\right)
$$

where $\Delta k_{n}(P V)$ are the values quoted in Table 3 at nominal values, $d_{n}=1 \mathrm{~cm}, R_{c n}$ and $\delta_{r n}=25 \mathrm{~cm}$. The values of the function $f\left(\frac{\delta_{r}}{\delta_{m}}\right)$ was taken from Fig. 4 due to the lack of better data. Because $d$ is proportional to $R_{c}$ (Eq. (37)), in general $\Delta k(P V)$ does not depend on $R_{c}$ and the iteration is not necessary. There is a lower limit to the reflector thickness, $0.4 \mathrm{~cm}$, a value from Ref. 4; when $d=0.4 \mathrm{~cm}$ an iteration on $R_{c}$ is then necessary.

Because of the effects of the pressure vessel on the worth of the $\mathrm{B}_{4} \mathrm{C}$ sheets a simple correlation was used to compute $\Delta k\left(B_{4} C\right)$ from the nominal values quoted in Table 4:

$$
\Delta k\left(B_{4} C\right)=A e^{-\Sigma d}\left(1-e^{-\Sigma_{c} d_{c}}\right) f\left(\frac{\delta_{r}}{\delta_{m}}\right)
$$

where the first exponential refers to the effects of pressure vessel of thickness $d$ and the second to the $B_{4} C$ sheet of thickness $d_{c}$; the third factor is related to the changes due to reflector thickness, like in Eq. (41). Equation (42) was calibrated with the data of Table 4 that were calculated for $d=1 \mathrm{~cm}$ and $d_{c}=2 \mathrm{~cm}$. 


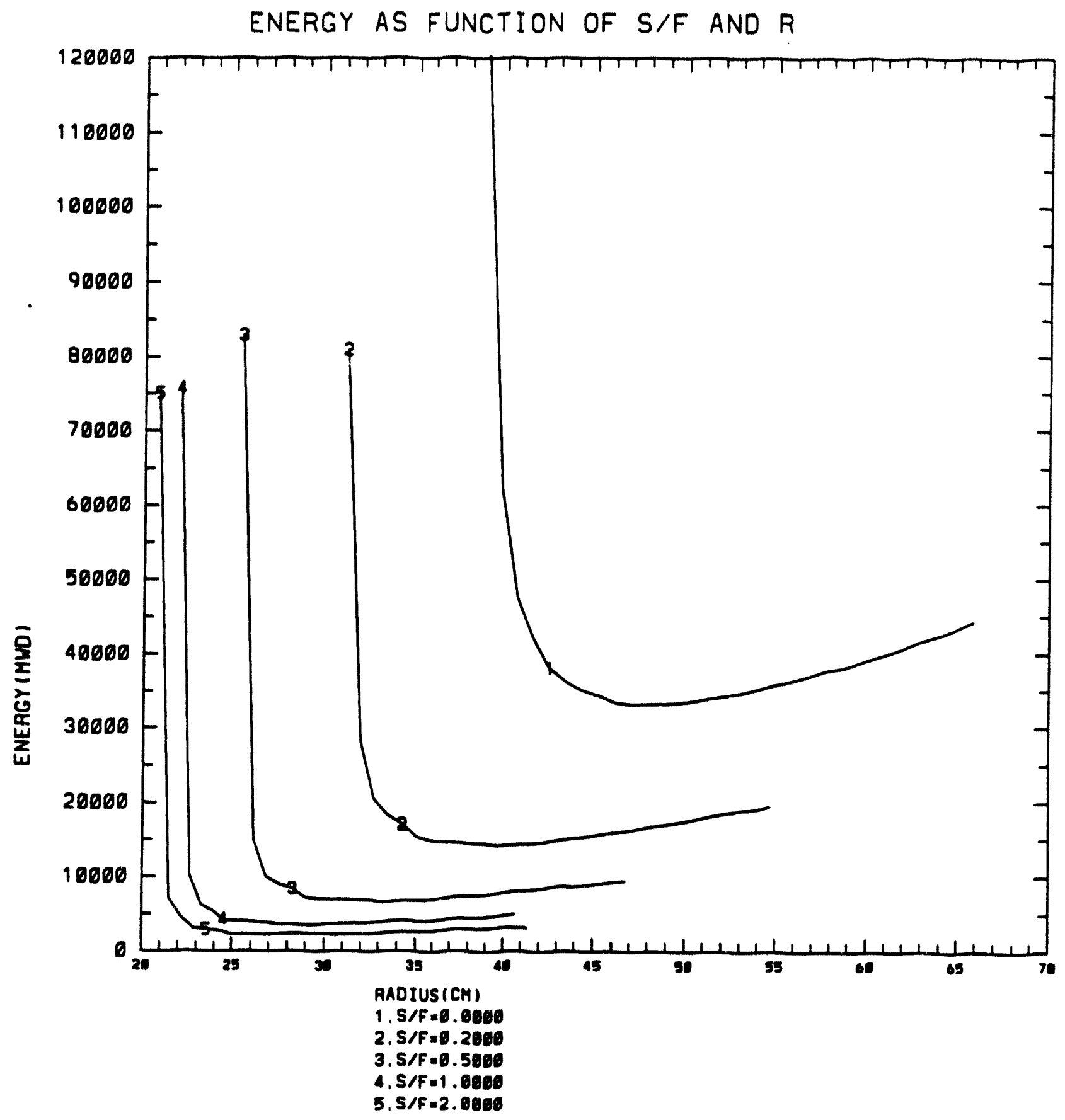

Fig. 12. Available energy in Mwd for the NERVA derivative reactor as a function of $S / F$, the radius of the core and for the following input conditions: $30 \mathrm{~cm}$ Be reflector, $2 \%$ reactivity worth for structural parts and desired end of life reactivity, and $15 \%$ burnup. 


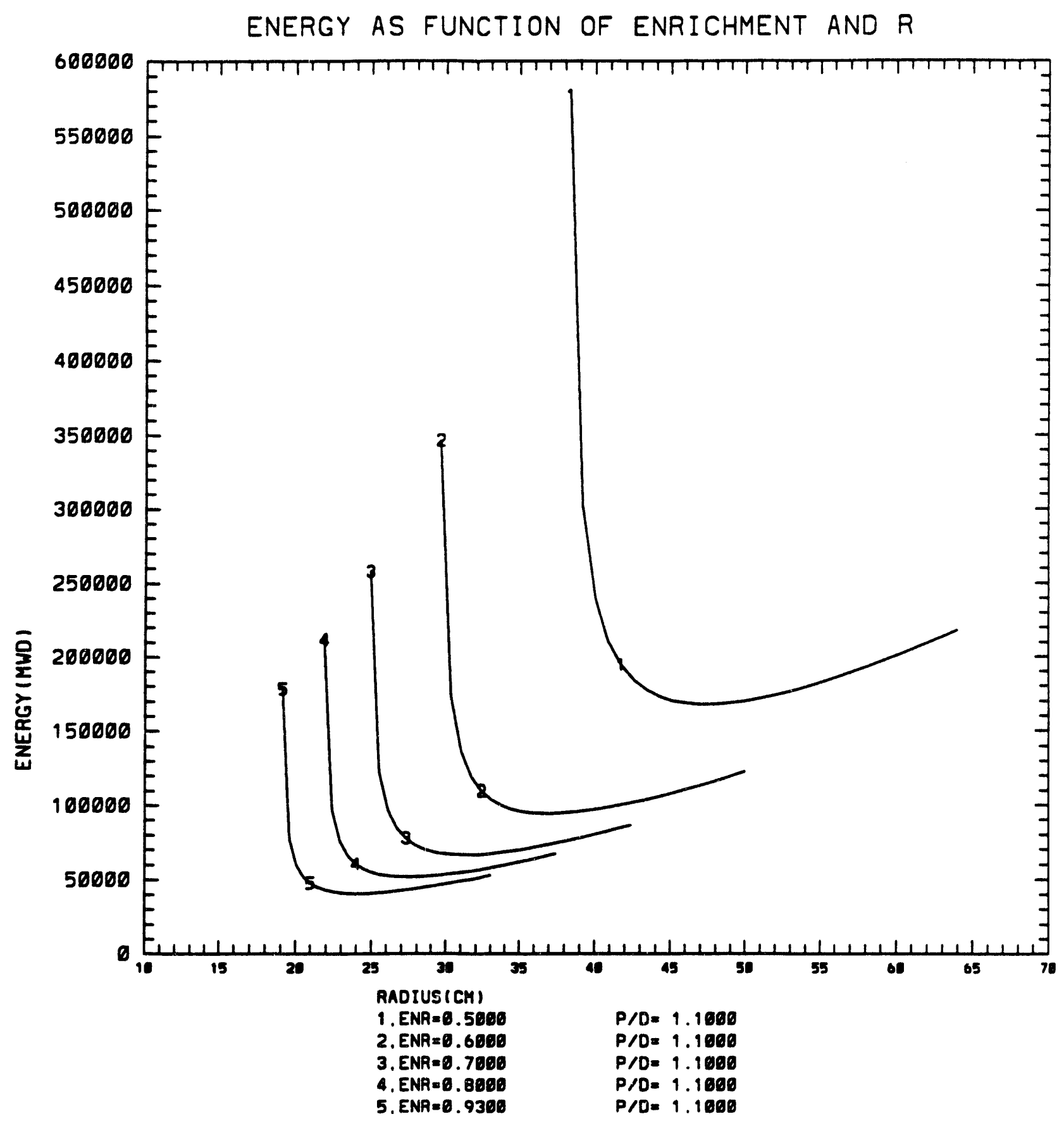

Fig. 13. Available energy in Mwd for the fuel pin reactor as a function of enrichment, the radius of the core and fixing $P / d=1.1$. Other fixed input variables are $30 \mathrm{~cm}$ of $\mathrm{BeO}$ reflector, $2 \%$ reactivity worth for structural parts and end of life desired reactivity and $15 \%$ burnup. 


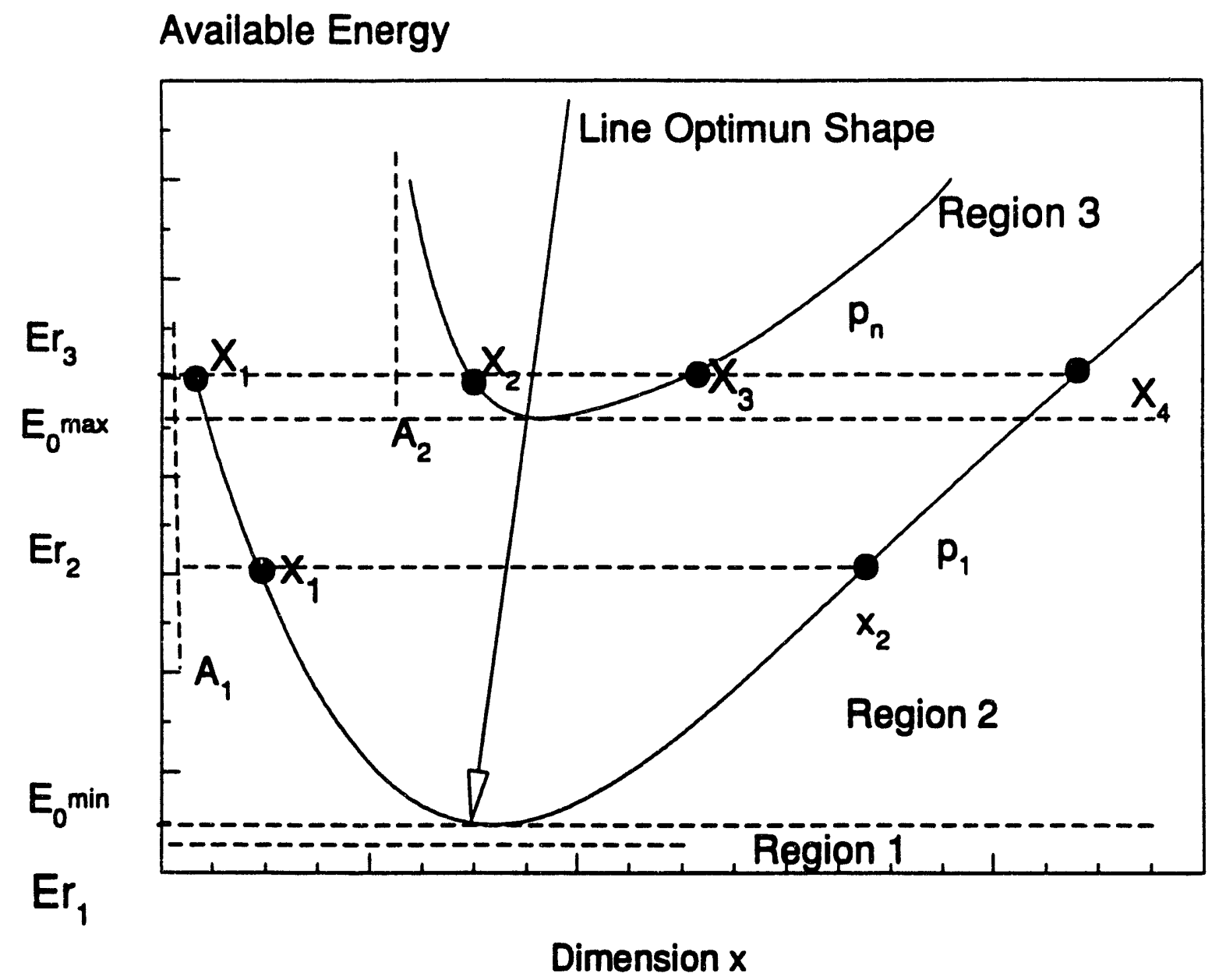

Fig. 14. Scheme for the exploration of possible designs, $E a$ is the available energy and $x$ is one of the core dimensions $\left(R_{c}\right.$ or $\left.H_{c}\right)$. 


\subsection{INPUT AND OUTPUT DESCRIPTIONS}

This section describes the input and output of the codes NEPNERVA and NEPPIN for, respectively, the helium cooled NERVA derivative and liquid lithium cooled fuel pin reactors. The names of the variables are those of the codes and in parenthesis some typical values.

\subsection{INPUT TO NEPNERVA}

itest/0,1/: Do not/run test, when itest $=1$ the code prints the energy available for the set of input conditions.

ndt: Number of points, in the tabulation of the available energy as function of $R_{c}$ or $H_{c}$, between the asymptotic and optimum values of $R_{c}$ or $H_{c}$.

itmax: Maximum number of iterations.

icont/01/Do not/continue calculation after test.

nput: Total number of points in the tabulation of the available energy.

Pow $(\mathrm{Mw})$ : $\quad$ Fission power.

$D$ (years): $\quad$ Time during which the reactor is on.

BU (\%): $\quad$ Burnup at end of life, i.e., percentage of ${ }^{235} \mathrm{U}$ atoms that disappeared by fission and capture.

eps (0.001): Iterations are stopped within this relative change.

der (1.01): Relative increment to compute derivatives.

drr $(\mathrm{cm})$ : Thickness of the radial Be retlector.

dral $(\mathrm{cm})$ : Thickness of the top axial Be reflector.

dra2 (cm): Thickness of the bottom axial Be reflector.

dkstr $(\%, 2)$ : Reactivity of structural components.

dkeol $(\%, 2)$ : Reactivity at end of life.

dksu $(\%, 2)$ : Subcritical reactivity with drums in.

For test runs only the input might finish here.

ncr: number of drums.

nnodo: number of axial nodes for thermalhydraulic calculations ( $\leq 50$ ).

nfu: number of radial nodes for temperature calculations of the fuel mixture.

ishape/1,2,3: Shape of the core

1: ideal shape $\left(\mathrm{R}_{\mathrm{c}} / \mathrm{H}_{\mathrm{c}}=0.54\right)$

2: input $R_{c}$

3: input $H_{c}$

iboun/0,1/: Input coolant flow/Outlet coolant temperature.

iopth/1,2,3,4/: Assumptions about the power and flow conditions

1: $3 D$ uniform power distribution.

2: $2 \mathrm{D}(r, \theta)$ uniform power distribution, cosine axial distribution ( 1 and 2 with uniform flow distribution).

3: $r, \theta$ power distribution corresponding to uniform fuel load, cosine axial distribution and uniform tlow distribution.

4: Like 3, but with $(r, \theta)$ tlow distribution p-oportional to power distribution.

iexpl/0,1/: No/Yes explicit thermalhydraulic, iexpl =! eliminates the iterations of Section 5.1.1.

ifri/0,1/: No/Yes inclusion of friction factors in pressure calculations.

iditus/0,1: No/Yes Dittus correlation for heat transfer coefticient, if iditus $=0$ there is a correction because of radial gradient of temperatures.

iprth/0,1/: No/Yes print details of thermalhydraulic calculations. 
iouth/1,2,3/: Printing of thermalhydraulic condition of

1: Maximum power density channel

2: Minimum power density channel

3: Both

iwrte/0,1/ No/Yes printing of iterations.

ishm/1,2/ Selection of neutron shielding

1: LiH stainless steel matrix

2: $\mathrm{B}_{4} \mathrm{C}$

ichoose 10,1/: For the case of contradictions between demand and availability:

0: Program chooses alternative demands.

1: Program stops for new demands from user (after printing some advices).

pin $(\mathrm{MPa}), \operatorname{tin}\left({ }^{\circ} \mathrm{K}\right)$ : Pressure and temperature at the inlet of reactor.

ps(MPa),ts $\left({ }^{\circ} \mathrm{K}\right)$ : Pressure and temperature in support elements, the thermalhydraulics of these elements are not calculated hy the code. ps and ts are used just to compute the He density.

flowt $(\mathrm{kg} / \mathrm{s})$ : Total coolant flow if ihoun $=0$.

tout $\left({ }^{\circ} \mathrm{K}\right)$ : Outlet temperature of coolant if iboun $=1$.

ru: rugosity of the coolant channel.

$\mathrm{xi}(1$.$) : x$ in Eq. (30).

fact(0.97): Factor for the conversion of fission power to thermal power.

ripple(0.1): Maximum ripple of the power distribution in the azimuthal direction (see Eq. (21)).

tempv $\left({ }^{\circ} \mathrm{K}\right)$ : Temperature of the pressure vessel, if input $<0$ program chooses toutl as the value of this variable.

depvw(cm): Maximum allowed for pressure vessel thickness.

fastf $\left(1 / \mathrm{cm}^{2}\right)$ : Maximum fast fluence at cargo hay.

dose(rad): Maximum gamma dose at cargo hay.

zload(m): Distance of cargo bay to base of reactor:

rload(m): Radius of cargo bay.

$\mathbf{R}(\mathrm{cm})$ : Radius of core if ishape $=1$.

$\mathbf{H}(\mathrm{cm})$ : Length of core if ishape $=3$.

The input file for the sample case follows. 
Problem 1 NERVA Application to NEP

itest/* dR/1tma/cont?/* of points in test Format is

$0 \quad 10 \quad 450 \quad 1 \quad 31$

Pow(Mw) /Life(y) /Burnup(8)/Tolerance/Rel delta El0.0

50. 2 2. 20 . $2001 \quad 1.01$

Radial /Top /Bottom Reflector Thickness(cm)

30. 0 . 0 .

struct. /End Life/Subcrit Reactivities(8)

2 . 2 . 2.

\#drum/\#axi/\#rad/ishape: $1,2,3$; ideal, choose $R$, choose $H$

$1250 \quad 3 \quad 1$

ibou/iopth: 1, 2, 3,4 (Power, flow conditions); iboun:0, 1 Boundary condition 13

Lexpl/ifri/idit/iprt/iouth/explicit, friction,Dittus, PrintDetails, Max, Min, Both?

iwrte/Nshield/Ichoose/wrte its, LiH-ss or B4C?. Ichoose or Program?

Pin(MPa) /Tin(K) /Pin /Tin/Inlet Conditions Fuel, support Elements

2. 400.

5. 400 .

iboun $=0$ input total coolant flow $(\mathrm{Kg} / \mathrm{s})$, iboun=1 input outlet $\mathrm{T}(\mathrm{K})$

1700 .

rugosity /Implicit /Fraction/P Ripple Angular Power

$0.011 .0 .97 \quad 0.1$

pressure vessel temperature/max permissible thickness $(\mathrm{cm})$

800.10.

Max fast $n / D O s e(R A D) / 2(m) \quad / r(m)$ shielding at $2, r$ for input dose

1. e+13 1000000.15 .0

5.

ishape/1,2,3/ (skip, input $R$, input $H(\mathrm{~cm})$ )

60 . 


\subsection{OUTPUT FROM NEPNERVA}

The output from running the code with the input file of the previous section follows.

Problem I NERVA Application to NEP

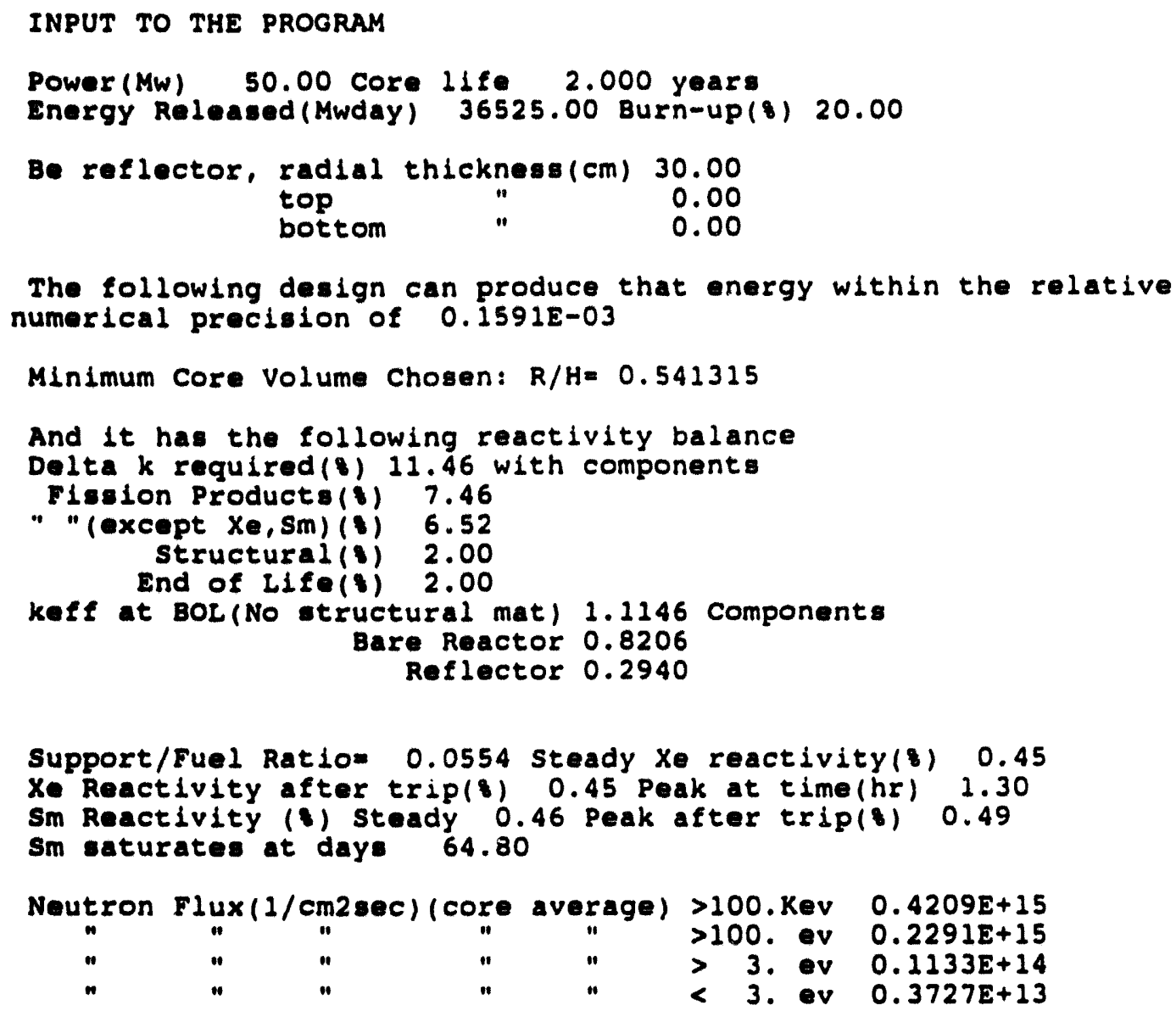

The following design can produce that energy within the relative numerical precision of $0.1591 \mathrm{E}-03$

Minimum Core Volume Chosen: $R / H=0.541315$

And it has the following reactivity balance

Delta $k$ required(\$) 11.46 with components

Fiseion Products( 7.46

" (except Xe, $\mathrm{sm}$ ) (\$) 6.52

structural (\$) 2.00

End of Iife(s) 2.00

keff at BoL (No structural mat) 1.1146 Components

Bare Reactor 0.8206

Reflector 0.2940

Support/Fuel Ratio= 0.0554 steady Xe reactivity(8) 0.45

Xe Reactivity after trip(8) 0.45 Peak at time(hr) 1.30

Sm Reactivity (\$) steady 0.46 Peak after trip(8) 0.49

$\mathrm{Sm}$ saturates at days 64.80

Neutron Flux (1/cm2 sec) (core average) $>100 . \mathrm{Kev} 0.4209 \mathrm{E}+15$

$"$ " " "

" " " " " $"$. ev $0.1133 E+14$

" " $"$ " $"$ o ev $0.3727 \mathrm{E}+13$

12 Drums with absorbing angle(d) 55.83 each Control dk(s)= 11.46

NUMBER DENSITIES AND MASSES OF NERVA DERIVATIVE REACTOR GENERAL DESCRIPTION

$265.412 \mathrm{~kg}$ of U235. U enrichment 0.9300 Core Volume(L) 560.24 Ratio Support/Fuel Elements 0.05542

Core Radius $(\mathrm{cm}) \quad 45.86 \mathrm{Height}(\mathrm{cm}) \quad 84.78$

Number of elements 2085 support 110 Fuel 1975

Hexagonal Elements. Flat to flat distance $(\mathrm{cm}) \quad 1.9130$

FUEL ELEMENTS (INDIVIDUAL)

Molar UC fraction in UC-2rC mix is $0.5000 \mathrm{zrC}$ clad thickness (mm) is 0.10

There are 19 coolant holes of $\mathrm{mm} 2.79$ diameter

with he coolant at $2.000 \mathrm{MPa} 1049.45 \mathrm{~K}$

Volume fractions in Fuel Element 
Fuel microcel1(Number Densities $10 * \star 24$ atoms $/ \mathrm{cm} 3$ ) Excludes outer cladding Coolant Radius $(\mathrm{cm}) 0.1397$ He 0.00013774 Clad Radius (cm) 0.1497 C 0.03908465 Cell Radius (cm) 0.2280 c 0.00494617 $2 r \quad 0.00247308$ Graphite $0.07316656 \quad$ u235 0.00229997 U238 0.00017312

FUEL ELEMENTS (ALL)

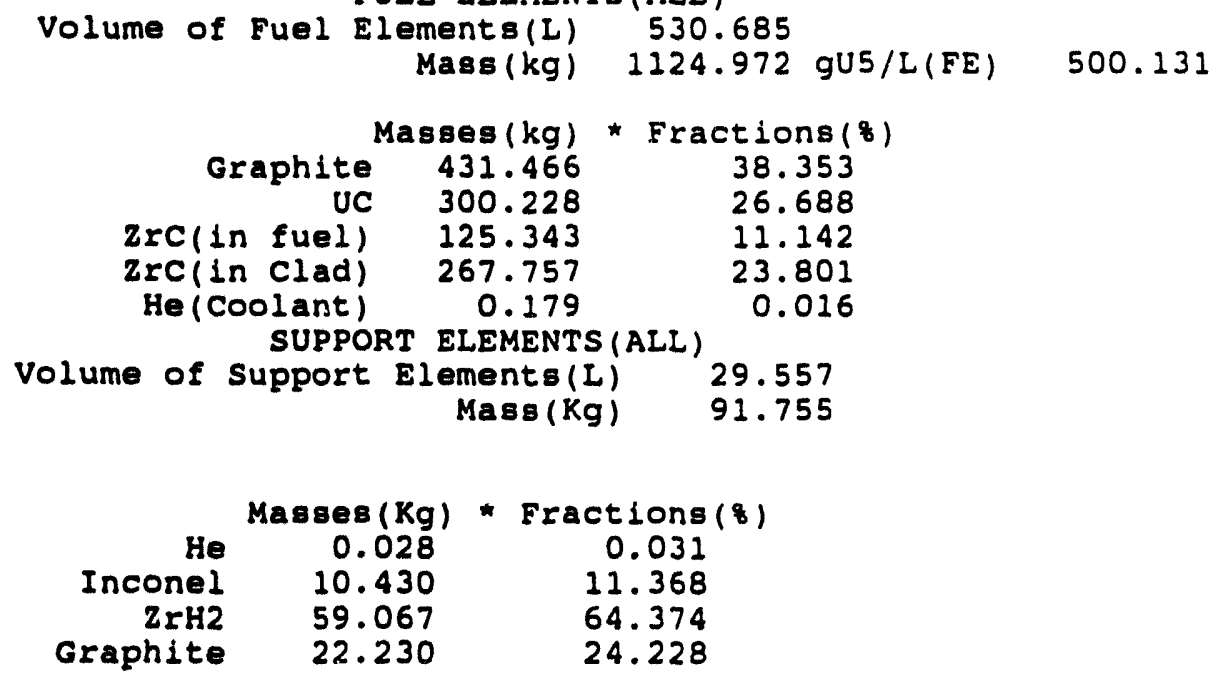

SUPERCELI CONFIGURATION(Number Densities $10 * \star 24$ atoms $/ \mathrm{cm} 3$ )

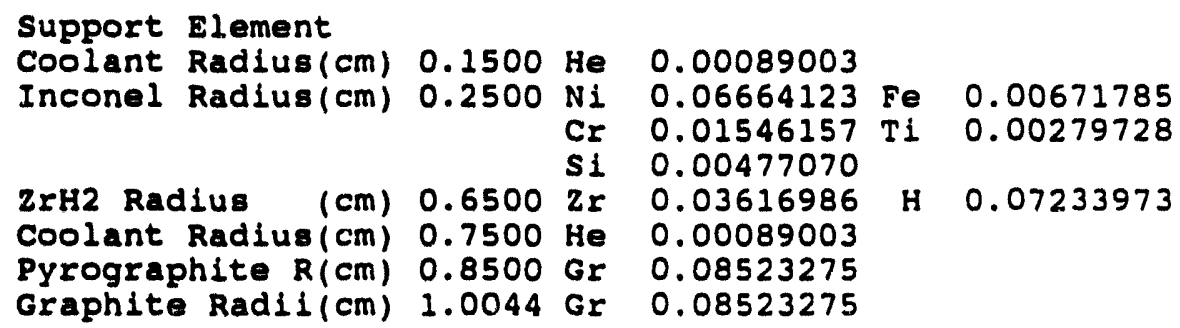

HOMOGENIZED DENSITIES IN CORE Radius (cm) 45.8626 
From Fuel Elements

$$
\begin{array}{llll}
\text { He } & 0.00004796 & \text { C } & 0.00539829 \\
\text { 2r } & 0.00409316 & \text { Gr } & 0.02861255 \\
\text { U5 } & 0.00121377 & \text { U8 } & 0.00009136
\end{array}
$$

From support Elements

$$
\begin{array}{llll}
\text { He } & 0.00000756 & \mathrm{Ni} & 0.00013941 \\
\mathrm{Fe} & 0.00001405 & \mathrm{Cr} & 0.00003234 \\
\mathrm{Ti} & 0.00000585 & \mathrm{Si} & 0.00000998 \\
\mathrm{Zr} & 0.00068097 & \mathrm{H} & 0.00136193 \\
\mathrm{Gr} & 0.00198940 & &
\end{array}
$$

REFLECTOR

External Radius(cm) 75.8626

Be 0.12348490

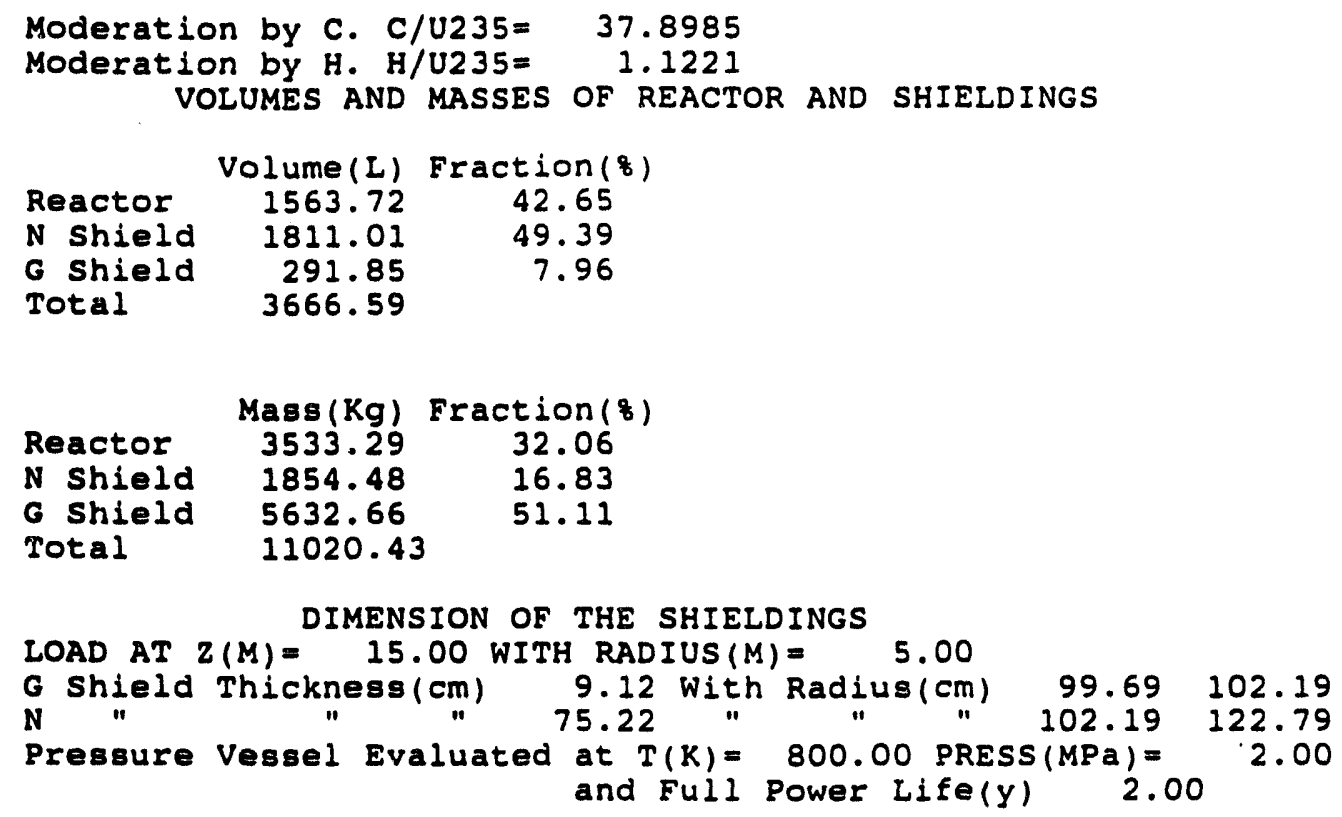


NEUTRON SHIELDING: LiH-Stainless steel Matrix

TOTAL PRODUCTION OF GAMMA RAYS(1/sEC)

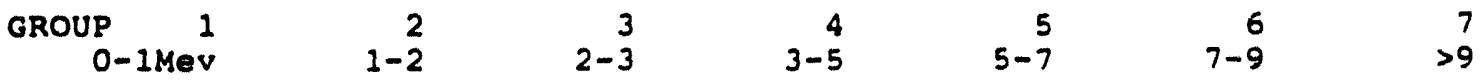

$2.084 E+19 \quad 6.404 E+18 \quad 2.452 E+18 \quad 9.606 E+17 \quad 2.708 E+17 \quad 1.207 E+16 \quad 3.077 E+16$

\& FRACTION PRODUCED BY U235

$\begin{array}{lllllll}98.09 & 95.67 & 94.01 & 63.32 & 24.66 & 0.00 & 79.38\end{array}$

Equivalent $z$ for Build up factors

$\begin{array}{lllllll}34.65 & 26.86 & 27.32 & 31.76 & 35.89 & 39.13 & 41.82\end{array}$

Gamma Self shielding by Core, Reflector and PV

$\begin{array}{llllllll}0.101662 & 0.193773 & 0.190531 & 0.212077 & 0.216133 & 0.215108 & 0.213580\end{array}$

The same but without Buildup factor

$\begin{array}{llllllll}0.048616 & 0.095106 & 0.109257 & 0.143415 & 0.159791 & 0.168326 & 0.171213\end{array}$

Integrated Dose(Rad) without any shielding( $n$ or gamma)

$1.380 E+09 \quad 1.984 E+09 \quad 9.781 E+08 \quad 5.971 E+08 \quad 2.422 E+08 \quad 1.352 E+07 \quad 3.852 E+07$

Integrated Dose with $W$ and Neutrons Shieldings

3.878E-02 9.709E+04 $1.984 E+05 \quad 4.868 E+05 \quad 1.967 E+05 \quad 6.884 E+03 \quad 1.370 E+04$

Integrated Dose without Any Shielding, Total(Rad) $0.5233 E+10$

Integrated dose with Shieldings ( $W$ and Neutrons), Total (Rads) $0.10 E+07$

Tungsten Thickness (cm) 9.12

Tungsten Thickness (cm) 11.65 if no Neutron Shield Present

Requested Dose(Rad) 1000000.00 at 15.00 meters

THERMALHYDRAULICS CALCULATIONS

Average Temperature of the Coolant at Core Exit(K) 1698.90

BOUNDARY CONDITION: OUTLET TEMPERATURE

****PROFILE OF CHANNEL WITH MAXIMUM POWER DENSITY****

Problem $\#$ Nerva Application to NEP

Thermodynamic profile of a He cooled channel

Inlet pressure(MPa) $=2.00000$ Inlet temperature $(\mathrm{K})=400.00$

Diameter $(\mathrm{cm})=0.28$ Rugosity $=0.01000$ der parm $=1.0100$ eps $=0.0010000$

Total Power (Mw) 50.000 No. Orifices 37525

Total Flow ( Kg/sec) 7.186

Factor that multiplies Power 0.9700000

Average Power Density(Mw/L of active mix) 0.1640 Channel Length $(\mathrm{cm}) \quad 84.78$

Cosine Axial Power Distribution=roav*Pfact*Cos (Pi*z/H)

Pfact $(A x i a l * R a d i a l * A z i m)=2.3949$ Extrapolated Delta $(\mathrm{cm}) \quad 5.00$

Attention Implicit Calculation!! $x i=1.00000$

Twall/Tbulk correction to Dittus-Boelter Correlation for $\mathrm{Nu}$ Uniform Coolant Flow Assumed

$\begin{array}{rrrrrrrr}\text { Flow }(g / s)= & 0.191 \\ \text { Node } & z(\mathrm{~cm}) & \text { Po }(\mathrm{w} / \mathrm{cm}) & \operatorname{Pr}(\mathrm{MPa}) & T(K) & V(\mathrm{~m} / \mathrm{s}) & \text { Re } & \text { Mach } \\ & & & & & & & \\ 1 & 0.00 & 6.02 & 2.00 & 400.00 & 13.06 & 3600 . & 0.011 \\ 2 & 1.70 & 8.04 & 2.00 & 411.99 & 13.46 & 3528 . & 0.011 \\ 3 & 3.39 & 10.02 & 2.00 & 427.39 & 13.97 & 3440 . & 0.011 \\ 4 & 5.09 & 11.98 & 2.00 & 446.15 & 14.59 & 3340 . & 0.012 \\ 5 & 6.78 & 13.90 & 2.00 & 468.21 & 15.31 & 3231 . & 0.012 \\ 6 & \mathbf{8 . 4 8} & 15.77 & 1.99 & 493.51 & 16.15 & 3116 . & 0.012\end{array}$

Pinge 8-7 


\begin{tabular}{|c|c|c|c|c|c|c|c|}
\hline $\begin{array}{l}7 \\
8 \\
9 \\
10 \\
11 \\
12 \\
13 \\
14 \\
15 \\
16 \\
17 \\
18 \\
19 \\
20 \\
21 \\
22 \\
23 \\
24 \\
25 \\
26 \\
27 \\
28 \\
29 \\
30 \\
31 \\
32 \\
33 \\
34 \\
35 \\
36 \\
37 \\
38 \\
39 \\
40 \\
41 \\
42 \\
43 \\
44 \\
45 \\
46 \\
47 \\
48 \\
49 \\
50 \\
51\end{array}$ & $\begin{array}{l}10.17 \\
11.87 \\
13.57 \\
15.26 \\
16.96 \\
18.65 \\
20.35 \\
22.04 \\
23.74 \\
25.43 \\
27.13 \\
28.83 \\
30.52 \\
32.22 \\
33.91 \\
35.61 \\
37.30 \\
39.00 \\
40.70 \\
42.39 \\
44.09 \\
45.78 \\
47.48 \\
49.17 \\
50.87 \\
52.57 \\
54.26 \\
55.96 \\
57.65 \\
59.35 \\
61.04 \\
62.74 \\
64.44 \\
66.13 \\
67.83 \\
69.52 \\
71.22 \\
72.91 \\
74.61 \\
76.30 \\
78.00 \\
79.70 \\
81.39 \\
83.09 \\
84.78\end{array}$ & $\begin{array}{r}17.60 \\
19.37 \\
21.07 \\
22.72 \\
24.29 \\
25.78 \\
27.19 \\
28.52 \\
29.75 \\
30.89 \\
31.94 \\
32.88 \\
33.72 \\
34.45 \\
35.08 \\
35.59 \\
35.99 \\
36.28 \\
36.45 \\
36.51 \\
36.45 \\
36.28 \\
35.99 \\
35.59 \\
35.08 \\
34.45 \\
33.72 \\
32.88 \\
31.94 \\
30.89 \\
29.75 \\
28.52 \\
27.19 \\
25.78 \\
24.29 \\
22.72 \\
21.07 \\
19.37 \\
17.60 \\
15.77 \\
13.90 \\
11.98 \\
10.02 \\
8.04 \\
6.02\end{array}$ & $\begin{array}{l}1.99 \\
1.99 \\
1.99 \\
1.99 \\
1.98 \\
1.98 \\
1.98 \\
1.98 \\
1.97 \\
1.97 \\
1.97 \\
1.97 \\
1.97 \\
1.97 \\
1.97 \\
1.97 \\
1.97 \\
1.97 \\
1.97 \\
1.97 \\
1.97 \\
1.97 \\
1.97 \\
1.96 \\
1.96 \\
1.96 \\
1.96 \\
1.96 \\
1.96 \\
1.96 \\
1.96 \\
1.96 \\
1.96 \\
1.96 \\
1.96 \\
1.96 \\
1.96 \\
1.96 \\
1.96 \\
1.96 \\
1.96 \\
1.96 \\
1.96 \\
1.96 \\
1.96\end{array}$ & $\begin{array}{r}521.97 \\
553.49 \\
587.97 \\
625.31 \\
665.39 \\
708.08 \\
753.24 \\
800.74 \\
850.42 \\
902.13 \\
955.70 \\
1010.96 \\
1067.74 \\
1125.87 \\
1185.14 \\
1245.39 \\
1306.41 \\
1368.02 \\
1430.03 \\
1492.22 \\
1554.42 \\
1616.42 \\
1678.02 \\
1739.04 \\
1799.27 \\
1858.54 \\
1916.65 \\
1973.42 \\
2028.66 \\
2082.21 \\
2133.90 \\
2183.56 \\
2231.04 \\
2276.18 \\
2318.85 \\
2358.91 \\
2396.23 \\
2430.70 \\
2462.20 \\
2490.64 \\
2515.92 \\
2537.98 \\
2556.73 \\
2572.12 \\
2584.10\end{array}$ & $\begin{array}{l}17.08 \\
18.13 \\
19.27 \\
20.50 \\
21.84 \\
23.26 \\
24.77 \\
26.36 \\
28.04 \\
29.79 \\
31.58 \\
33.41 \\
35.28 \\
37.20 \\
39.16 \\
41.15 \\
43.16 \\
45.20 \\
47.25 \\
49.31 \\
51.37 \\
53.42 \\
55.46 \\
57.48 \\
59.48 \\
61.45 \\
63.38 \\
65.27 \\
67.11 \\
68.89 \\
70.61 \\
72.27 \\
73.86 \\
75.37 \\
76.80 \\
78.14 \\
79.40 \\
80.56 \\
81.62 \\
82.59 \\
83.45 \\
84.20 \\
84.85 \\
85.38 \\
85.80\end{array}$ & $\begin{array}{l}2998 . \\
2880 . \\
2763 . \\
2648 . \\
2538 . \\
2432 . \\
2331 . \\
2235 . \\
2144 . \\
2059 . \\
1979 . \\
1904 . \\
1834 . \\
1768 . \\
1707 . \\
1650 . \\
1596 . \\
1547 . \\
1500 . \\
1457 . \\
1417 . \\
1379 . \\
1344 . \\
1312 . \\
1281 . \\
1253 . \\
1227 . \\
1203 . \\
1180 . \\
1159 . \\
1140 . \\
1122 . \\
1105 . \\
1090 . \\
1076 . \\
1064 . \\
1052 . \\
1042 . \\
1033 . \\
1025 . \\
1018 . \\
1012 . \\
1007 . \\
1002 . \\
999 .\end{array}$ & $\begin{array}{l}0.013 \\
0.013 \\
0.013 \\
0.014 \\
0.014 \\
0.015 \\
0.015 \\
0.016 \\
0.016 \\
0.017 \\
0.017 \\
0.018 \\
0.018 \\
0.019 \\
0.019 \\
0.020 \\
0.020 \\
0.021 \\
0.021 \\
0.022 \\
0.022 \\
0.023 \\
0.023 \\
0.023 \\
0.024 \\
0.024 \\
0.025 \\
0.025 \\
0.025 \\
0.026 \\
0.026 \\
0.026 \\
0.027 \\
0.027 \\
0.027 \\
0.027 \\
0.027 \\
0.028 \\
0.028 \\
0.028 \\
0.028 \\
0.028 \\
0.029 \\
0.029 \\
0.029\end{array}$ \\
\hline
\end{tabular}

From the coolant Channel to the Uranium carbide

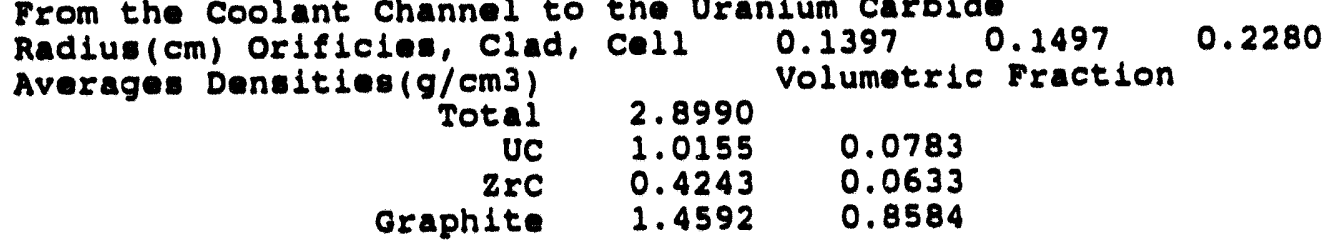

Number of tuel nodes 3

265411.72 grams of $U 235$ in 530.68 Liters of Fuel volume

\begin{tabular}{|c|c|c|c|c|c|c|c|}
\hline Node & $z(\mathrm{~cm})$ & $J(K w / c m 2)$ & Twall(K) & $\operatorname{Tclad}(K)$ & TCladAv & Tfuel (K) & TEuelav \\
\hline $\begin{array}{l}1 \\
2 \\
3 \\
4\end{array}$ & $\begin{array}{r}0.00 \\
1.70 \\
3.39 \\
5.09 \\
6.78 \\
8.48 \\
10.17\end{array}$ & $\begin{array}{l}0.00686 \\
0.00915 \\
0.01142 \\
0.01365 \\
0.01583 \\
0.01797 \\
0.02005\end{array}$ & $\begin{array}{l}467.46 \\
506.76 \\
559.58 \\
613.05 \\
668.55 \\
725.73 \\
783.95\end{array}$ & $\begin{array}{l}467.78 \\
507.19 \\
560.11 \\
613.69 \\
669.28 \\
726.56 \\
784.89\end{array}$ & $\begin{array}{l}467.63 \\
506.98 \\
559.85 \\
613.37 \\
668.92 \\
726.15 \\
784.43\end{array}$ & $\begin{array}{l}468.08 \\
507.62 \\
560.70 \\
614.41 \\
670.17 \\
727.62 \\
786.13\end{array}$ & $\begin{array}{l}467.99 \\
507.49 \\
560.52 \\
614.20 \\
669.90 \\
727.31 \\
785.76\end{array}$ \\
\hline
\end{tabular}

Page 8.8 


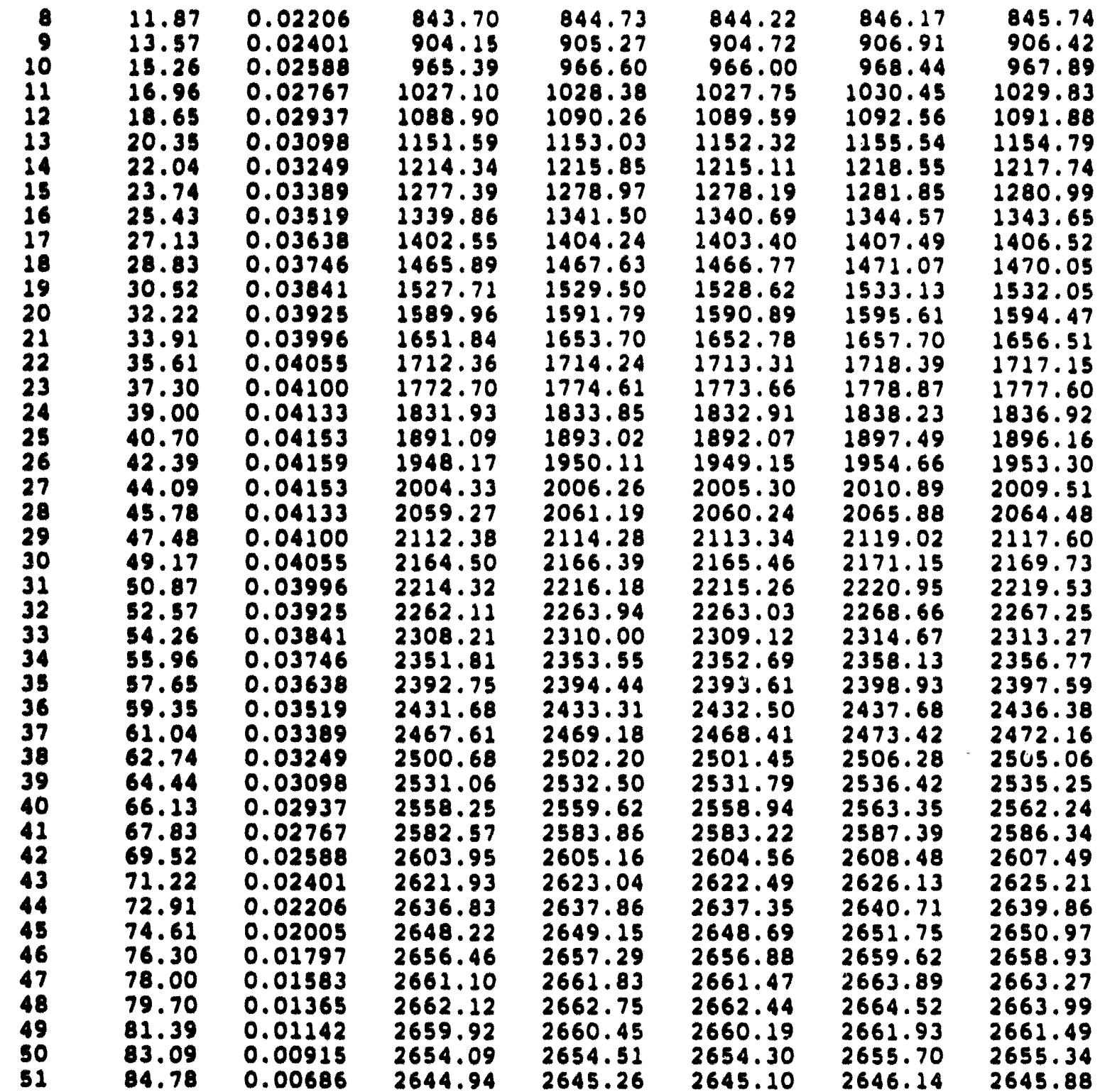

Axilal Average Conditione in the Channel

Coolant Pressure(kPa, 1.970 Temperature(K) 1056.332

Give Density $(\mathrm{g} / \mathrm{cm} 3) 0.000896 j$

Average Deneity 0.0008960

Wall Temperature(K) 1815.332

Clad Tomperature(K) 1816.019

Tuei Temperature(K) 1818.830

Mol Fraction of UC in UC-2rC mix0.50000 Melting Temperature(K) 3343.50

HEAT BALANCE TOR THE CHANNEL

do: heat to coolant (joule $/ \mathrm{kg}$ ) $0.11343 \mathrm{E}+08$

dit change of enthalpy (joule/kg) $0.11340 E+08$

dKin change klnetic onergy (joule/kg) $0.35955 \mathrm{E}+04$

relative dKin/do 0.000317

Balance $(d H+d K I n) / d Q \quad 1.0000000$

"\#"PROFILE OF CHANNEL WITH MINIMUM POWER DENSITY**" 
Problem I Nerva Applleation to NEP

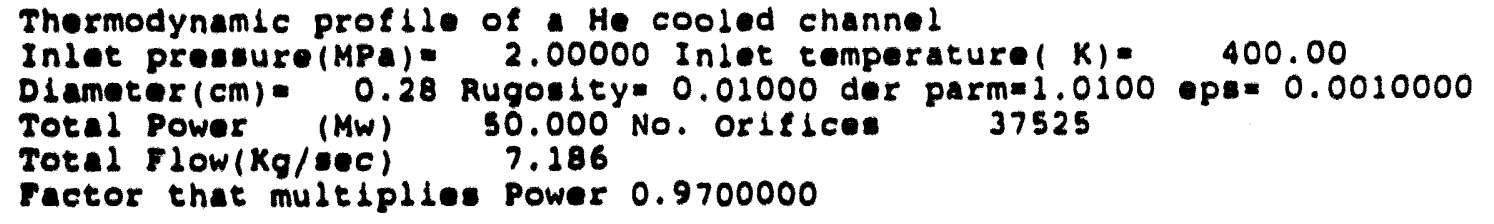

Average Power Density (Mw/L of active $\mathrm{mix}$ ) 0.1640 Channel Length $(\mathrm{cm}) \quad 84.78$

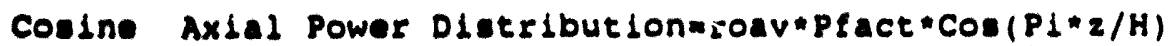

Plact (Axlal*Radlal Azim)=0.6851 Extrapolated nelta(cm) 5.00

Attention Implicit calculation!l $\therefore$ is 1.00000

Twall/Tbulk correction to Dittus-Boelter correlation for Nu Unteorm Coolant Flow Asaumed

\begin{tabular}{|c|c|c|c|c|c|c|c|}
\hline Flow & 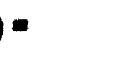 & 91 & & & & & \\
\hline Node & $2(\mathrm{~cm})$ & $P O(w / \mathrm{cm})$ & $\operatorname{Pr}(\mathrm{MPa})$ & $T(K)$ & $V(m / s)$ & Re & Mach \\
\hline $\begin{array}{r}1 \\
2 \\
3 \\
4 \\
5 \\
6 \\
7 \\
8 \\
9 \\
10 \\
11 \\
12 \\
13 \\
14 \\
15 \\
16 \\
17 \\
18 \\
19 \\
20 \\
21 \\
22 \\
23 \\
24 \\
25 \\
26 \\
27 \\
28 \\
29 \\
30 \\
31 \\
32 \\
33 \\
34 \\
35 \\
36 \\
37 \\
38 \\
39 \\
40 \\
41 \\
42 \\
43 \\
44\end{array}$ & $\begin{array}{r}0.00 \\
1.70 \\
3.39 \\
5.09 \\
6.78 \\
8.48 \\
10.17 \\
11.87 \\
13.57 \\
15.26 \\
16.96 \\
18.65 \\
20.35 \\
22.04 \\
23.74 \\
25.43 \\
27.13 \\
28.83 \\
30.52 \\
32.22 \\
33.91 \\
35.61 \\
37.30 \\
39.00 \\
40.70 \\
42.39 \\
44.09 \\
45.78 \\
47.48 \\
49.17 \\
50.87 \\
52.57 \\
54.26 \\
55.96 \\
57.65 \\
59.35 \\
61.04 \\
62.74 \\
64.44 \\
66.13 \\
67.83 \\
69.52 \\
71.22 \\
72.91\end{array}$ & $\begin{array}{r}1.72 \\
2.30 \\
2.87 \\
3.43 \\
3.98 \\
4.51 \\
5.03 \\
5.54 \\
6.03 \\
6.50 \\
6.95 \\
7.37 \\
7.78 \\
8.16 \\
8.51 \\
8.84 \\
9.14 \\
9.41 \\
9.65 \\
9.86 \\
10.03 \\
10.18 \\
10.30 \\
10.38 \\
10.43 \\
10.44 \\
10.43 \\
10.38 \\
10.30 \\
10.18 \\
10.03 \\
9.86 \\
9.65 \\
9.41 \\
9.14 \\
8.84 \\
8.51 \\
8.16 \\
7.78 \\
7.37 \\
6.95 \\
6.50 \\
6.03 \\
5.54\end{array}$ & $\begin{array}{l}2.00 \\
2.00 \\
2.00 \\
2.00 \\
2.00 \\
1.99 \\
1.99 \\
1.99 \\
1.99 \\
1.99 \\
1.99 \\
1.99 \\
1.98 \\
1.98 \\
1.98 \\
1.98 \\
1.98 \\
1.98 \\
1.97 \\
1.97 \\
1.97 \\
1.97 \\
1.97 \\
1.96 \\
1.96 \\
1.96 \\
1.96 \\
1.95 \\
1.95 \\
1.95 \\
1.94 \\
1.94 \\
1.94 \\
1.94 \\
1.93 \\
1.93 \\
1.92 \\
1.92 \\
1.92 \\
1.91 \\
1.91 \\
1.91 \\
1.91 \\
1.91\end{array}$ & $\begin{array}{l}400.00 \\
403.43 \\
407.84 \\
413.20 \\
419.52 \\
426.75 \\
434.89 \\
443.91 \\
453.77 \\
464.46 \\
475.92 \\
488.13 \\
501.05 \\
514.64 \\
528.85 \\
543.65 \\
558.97 \\
574.78 \\
591.03 \\
607.66 \\
624.62 \\
641.86 \\
659.32 \\
676.95 \\
694.69 \\
712.48 \\
730.28 \\
748.02 \\
765.65 \\
783.11 \\
800.35 \\
817.31 \\
833.94 \\
850.18 \\
865.99 \\
881.32 \\
896.11 \\
910.32 \\
923.91 \\
936.83 \\
949.04 \\
960.51 \\
971.19 \\
981.05\end{array}$ & $\begin{array}{l}13.06 \\
13.18 \\
13.33 \\
13.52 \\
13.73 \\
13.97 \\
14.24 \\
14.55 \\
14.88 \\
15.24 \\
15.62 \\
16.03 \\
16.46 \\
16.92 \\
17.40 \\
17.90 \\
18.42 \\
18.95 \\
19.50 \\
20.07 \\
20.65 \\
21.24 \\
21.84 \\
22.44 \\
23.06 \\
23.68 \\
24.30 \\
24.92 \\
25.54 \\
26.16 \\
26.77 \\
27.38 \\
27.98 \\
28.58 \\
29.16 \\
29.72 \\
30.28 \\
30.82 \\
31.34 \\
31.84 \\
32.28 \\
32.67 \\
33.04 \\
33.38\end{array}$ & $\begin{array}{l}3600 . \\
3579 . \\
3552 . \\
3520 \\
3484 . \\
3443 . \\
3399 . \\
3351 . \\
3301 . \\
3249 . \\
3195 . \\
3140 . \\
3084 . \\
3028 . \\
2972 . \\
2916 . \\
2861 . \\
2806 . \\
2753 . \\
2701 . \\
2650 . \\
2601 . \\
2554 . \\
2508 . \\
2464 . \\
2421 . \\
2381 . \\
2342 . \\
2305 . \\
2269 . \\
2235 . \\
2203 . \\
2173 . \\
2145 . \\
2118 . \\
2092 . \\
2068 . \\
2046 . \\
2025 . \\
2006 . \\
1988 . \\
1972 . \\
1957 . \\
1944 .\end{array}$ & $\begin{array}{l}0.011 \\
0.011 \\
0.011 \\
0.011 \\
0.011 \\
0.011 \\
0.012 \\
0.012 \\
0.012 \\
0.012 \\
0.012 \\
0.012 \\
0.012 \\
0.013 \\
0.013 \\
0.013 \\
0.013 \\
0.013 \\
0.014 \\
0.014 \\
0.014 \\
0.014 \\
0.014 \\
0.015 \\
0.015 \\
0.015\end{array}$ \\
\hline
\end{tabular}

Page 8-10 


$\begin{array}{llllllll}45 & 74.61 & 5.03 & 1.91 & 990.07 & 33.68 & 1931 . & 0.018 \\ 46 & 76.30 & 4.51 & 1.91 & 998.21 & 33.96 & 1921 . & 0.018 \\ 47 & 78.00 & 3.98 & 1.91 & 1005.44 & 34.21 & 1911 . & 0.018 \\ 48 & 79.70 & 3.43 & 1.91 & 1011.75 & 34.42 & 1903 . & 0.018 \\ 49 & 81.39 & 2.87 & 1.91 & 1017.12 & 34.61 & 1896 . & 0.018 \\ 50 & 83.09 & 2.30 & 1.91 & 1021.53 & 34.76 & 1890 . & 0.018 \\ 51 & 84.78 & 1.72 & 1.91 & 1024.95 & 34.88 & 1886 . & 0.018\end{array}$

From the Coolant Channel to the Uranium Carbide

Radius(cm) Oriflces, Clad, Cell $0.1397 \quad 0.1497 \quad 0.2280$

Averages Denaities (g/ $\mathrm{cm} 3$ )

$$
\begin{array}{rll}
\text { Total } & 2.8990 & \\
\text { UC } & 1.0155 & 0.0783 \\
\text { zrC } & 0.4243 & 0.0633 \\
\text { aphite } & 1.4592 & 0.8584
\end{array}
$$

Number of furl nodes 3

\begin{tabular}{|c|c|c|c|c|c|c|c|}
\hline lode & $2(\mathrm{Cln})$ & $J(K w / c m 2)$ & Twall (K) & $\operatorname{Tclad}(K)$ & TCladAv & Tfuel $(K)$ & Tfuelav \\
\hline $\begin{array}{l}1 \\
2 \\
3 \\
4 \\
5 \\
6 \\
7 \\
8 \\
9 \\
10 \\
11 \\
12 \\
13 \\
14 \\
15 \\
16 \\
17 \\
18 \\
19 \\
20 \\
21 \\
22 \\
23 \\
24 \\
25 \\
26 \\
27 \\
28 \\
29 \\
30 \\
31 \\
32 \\
33 \\
34 \\
35 \\
36 \\
37 \\
38 \\
39 \\
40 \\
41 \\
42 \\
43 \\
44 \\
45 \\
46\end{array}$ & $\begin{array}{l}0.100 \\
1.70 \\
3.39 \\
5.09 \\
6.78 \\
8.48 \\
10.17 \\
11.87 \\
13.57 \\
15.26 \\
16.96 \\
18.65 \\
20.35 \\
22.04 \\
23.74 \\
25.43 \\
27.13 \\
28.83 \\
30.52 \\
32.22 \\
33.91 \\
35.61 \\
37.30 \\
39.00 \\
40.70 \\
42.39 \\
44.09 \\
45.78 \\
47.48 \\
49.17 \\
50.87 \\
52.57 \\
54.26 \\
55.96 \\
57.65 \\
59.35 \\
61.04 \\
62.74 \\
64.44 \\
66.13 \\
67.83 \\
69.52 \\
71.22 \\
72.91 \\
74.61 \\
76.30\end{array}$ & $\begin{array}{l}0.00196 \\
0.00262 \\
0.00327 \\
0.00390 \\
0.00453 \\
0.00514 \\
0.00573 \\
0.00631 \\
0.00687 \\
0.00740 \\
0.00791 \\
0.00840 \\
0.00886 \\
0.00929 \\
0.00970 \\
0.01007 \\
0.01041 \\
0.01072 \\
0.01099 \\
0.01123 \\
0.01143 \\
0.01160 \\
0.01173 \\
0.01182 \\
0.01188 \\
0.01190 \\
0.01188 \\
0.01182 \\
0.01173 \\
0.01160 \\
0.01143 \\
0.01123 \\
0.01099 \\
0.01072 \\
0.01041 \\
0.01007 \\
0.00970 \\
0.00929 \\
0.00886 \\
0.00840 \\
0.00791 \\
0.00740 \\
0.00687 \\
0.00631 \\
0.00573 \\
0.00514\end{array}$ & $\begin{array}{l}418.43 \\
428.86 \\
442.72 \\
456.75 \\
471.36 \\
486.64 \\
502.58 \\
519.20 \\
536.39 \\
553.97 \\
572.06 \\
590.41 \\
608.97 \\
627.86 \\
647.02 \\
666.19 \\
685.31 \\
704.51 \\
723.52 \\
742.50 \\
761.38 \\
779.88 \\
798.41 \\
816.44 \\
833.88 \\
851.21 \\
867.84 \\
884.27 \\
900.17 \\
915.21 \\
930.79 \\
944.52 \\
957.87 \\
970.33 \\
981.99 \\
992.99 \\
1003.13 \\
1012.38 \\
1020.71 \\
1028.09 \\
1034.51 \\
1040.09 \\
1044.67 \\
1048.23 \\
1050.91 \\
1052.46\end{array}$ & $\begin{array}{l}418.52 \\
428.98 \\
442.87 \\
456.93 \\
471.57 \\
486.88 \\
502.85 \\
519.49 \\
536.71 \\
554.32 \\
572.43 \\
590.81 \\
609.39 \\
628.29 \\
647.47 \\
666.66 \\
685.79 \\
705.01 \\
724.04 \\
743.02 \\
761.92 \\
780.42 \\
798.96 \\
816.99 \\
834.44 \\
851.76 \\
868.40 \\
884.82 \\
900.71 \\
915.75 \\
931.33 \\
945.04 \\
958.38 \\
970.83 \\
982.48 \\
993.46 \\
1003.58 \\
1012.81 \\
1021.12 \\
1028.48 \\
1034.88 \\
1040.44 \\
1044.99 \\
1048.52 \\
1051.17 \\
1052.70\end{array}$ & $\begin{array}{l}418.47 \\
428.92 \\
442.79 \\
456.84 \\
471.47 \\
486.76 \\
502.72 \\
519.35 \\
536.55 \\
554.15 \\
572.25 \\
590.61 \\
609.18 \\
628.08 \\
647.25 \\
666.43 \\
685.55 \\
704.77 \\
723.78 \\
742.76 \\
761.65 \\
780.15 \\
798.69 \\
816.72 \\
834.16 \\
851.49 \\
868.12 \\
884.54 \\
900.44 \\
915.48 \\
931.06 \\
944.78 \\
958.13 \\
970.58 \\
982.24 \\
993.23 \\
1003.36 \\
1012.59 \\
1020.92 \\
1028.29 \\
1034.69 \\
1040.27 \\
1044.83 \\
1048.38 \\
1051.04 \\
1052.58\end{array}$ & $\begin{array}{r}418.60 \\
429.09 \\
443.01 \\
457.10 \\
471.77 \\
487.11 \\
503.12 \\
519.79 \\
537.05 \\
554.69 \\
572.83 \\
591.24 \\
609.86 \\
628.79 \\
648.00 \\
667.22 \\
686.38 \\
705.63 \\
724.68 \\
743.69 \\
762.61 \\
781.14 \\
799.70 \\
817.75 \\
835.21 \\
852.55 \\
869.19 \\
885.61 \\
901.51 \\
916.55 \\
932.12 \\
945.83 \\
959.16 \\
971.59 \\
983.23 \\
994.19 \\
1004.29 \\
1013.49 \\
1021.78 \\
1029.11 \\
1035.47 \\
1041.00 \\
1045.51 \\
1049.00 \\
1051.61 \\
1053.09\end{array}$ & $\begin{array}{l}418.58 \\
429.06 \\
442.97 \\
457.05 \\
471.71 \\
487.04 \\
503.04 \\
519.70 \\
536.95 \\
554.58 \\
572.71 \\
591.11 \\
609.72 \\
628.64 \\
647.84 \\
667.06 \\
686.21 \\
705.45 \\
724.49 \\
743.49 \\
762.40 \\
780.92 \\
799.48 \\
817.52 \\
834.98 \\
852.31 \\
868.95 \\
885.38 \\
901.27 \\
916.31 \\
931.88 \\
945.59 \\
958.93 \\
971.36 \\
983.01 \\
993.97 \\
1004.08 \\
1013.29 \\
1021.58 \\
1028.92 \\
1035.29 \\
1040.83 \\
1045.36 \\
1048.86 \\
1051.48 \\
1052.97\end{array}$ \\
\hline
\end{tabular}

265411.72 grams of $U 235$ in 530.68 Liters of Fuel volume

Page 8-11 


$\begin{array}{llllllll}47 & 78.00 & 0.00453 & 1053.04 & 1053.25 & 1053.15 & 1053.60 & 1053.49 \\ 48 & 79.70 & 0.00390 & 1052.65 & 1052.83 & 1052.74 & 1053.13 & 1053.04 \\ 49 & 81.39 & 0.00327 & 1051.20 & 1051.35 & 1051.27 & 1051.60 & 1051.52 \\ 50 & 83.09 & 0.00262 & 1048.73 & 1048.85 & 1048.79 & 1049.05 & 1048.99 \\ 51 & 84.78 & 0.00196 & 1045.25 & 1045.34 & 1045.29 & 1045.49 & 1045.44\end{array}$

Axial Average Conditions in the Channel

Coolant Pressure(MPa) 1.954 Temperature(K) 643.987

Give Density $(\mathrm{g} / \mathrm{cm} 3) \quad 0.0014554$

Average Density 0.0014553

wall Temperature(K) 808.533

Clad Temperature (K) 808.729

Fuel Temperature(K) 809.296

Mol Fraction of UC in UC-2rC mix0.50000 Melting Temperature(K)

3343.50

HEAT BALANCE FOR THE CHANNEL

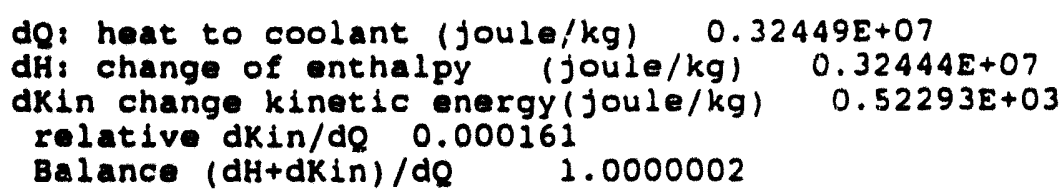




\subsection{INPUT TO NEPPIN}

itest/0,1/: Do not/run test, when itest $=1$, the code prints the energy available for the set of input conditions.

ndt: Number of points, in the tabulation of the available energy as function of $R_{c}$ or $H_{c}$, between the asymptotic and optimum values of $R_{c}$ or $H_{c}$.

itmas: Maximum number of iterations.

icont/0,1/ Do not/continue calculation after test.

iboun/1,2/: Input coolant flow/outlet coolant temperature.

nput: Total of points in the tabulation of the available energy.

Pow(Mw): Fission power.

$D$ (years): Time during which the reactor is on.

BU(\%): Burnup at end of life, see Eq. (3).

eps(0.001): Iterations are stopped within this relative thange.

der(1.01): Relative increment to compute derivatives.

Jrr(cm): Thickness of the radial Be reflector.

dral $(\mathrm{cm})$ : Thickness of the top axial Be reflector.

dra2(cm): Thickness of the bottom axial Be reflector.

tduc(mm): Thickness of the duct of the hundle.

dkstr $(\%, 2)$ : Reactivity of structural components.

dkeol $(\%, 2)$ : Reactivity at end of life.

dksu(\%,2): Subcritical reactivity with drums in.

pin $(\mathrm{MPa}), \operatorname{tin}\left({ }^{\circ} \mathrm{K}\right)$ : Pressure and temperature at the inlet of reactor.

$\mathrm{pHe}(\mathrm{MPa}), \mathrm{THe}(\mathrm{K})$ : Pressure and temperature of the $\mathrm{He}$ in the gap of the fuel rod.

tempv $\left({ }^{\circ} \mathrm{K}\right)$ : Temperature of the pressure vessel, if input $<0$ program chooses toutl as the value of this variable.

flowt $(\mathrm{Kg} / \mathrm{s})$ : Total coolant flow if iboun $=1$.

tout $\left({ }^{\circ} \mathrm{K}\right)$ : Outlet temperature of coolant if ihoun $=2$.

User might stop input here for a test only run.

ncr: Number of drums.

nnodo: Number of axial nodes for thermalhydraulic calculations $(\leq 50)$.

npin: Number of radial nodes for temperature calculations in fuel pin.

ishape/1,2,3/: Shape of the core.

1: Ideal shape $\left(\mathrm{R}_{\mathrm{c}} / \mathrm{H}_{\mathrm{c}}=0.54\right)$.

2: Input $R_{c}$

3: Input $\mathrm{H}_{\mathrm{c}}$

iopth/1,2,3,4/: Assumptions about the power and flow conditions.

1: 3D uniform power distributions.

2: $2 D(r, \theta)$ uniform power distribution, cosine axial distribution (1 and 2 with uniform flow distribution.

3: r, $\theta$ power distribution corresponding to uniform fuel load. cosine axial distribution and uniform flow distribution.

4: Like 3, but with $(r, \theta)$ flow distribution proportional to power distrihution.

ifri/0,1/: No/Yes Inclusion of friction factors in pressure calculations.

iprth/0,1/: No/Yes Print details of thermalhydraulic calculations.

iouth/1,2,3/: Printing of thermalhydraulic condition of

1: Maximum power density channel.

2: Minimum power density channel.

3: Both.

iwrte/0,1/ No/Yes Printing of iterations. 
ishn/1,2/ Selection of neutron shielding.

1: LiH in stainless steel matrix.

2: $\mathrm{B}_{4} \mathrm{C}$

ichoose/0,1/: For the case of contradictions between demand and availability:

0: Program chooses alternative demands.

1: Program stops for new demands from user (after printing some advices).

Ichoode/ $0,1 \ldots 5 /$ : Selection of a design

5 designs are calculated for $P / d=1.1,1.2,1.3,1.4$. and 1.5. If ichoode $=0$ the code selects a design based on the $\Delta p$ along the channel and the total mass: the user can override the selection process by choosing one of the five.

fact(0.97): Factor for the conversion of fission power to thermal power.

ripple(0.1): Maximum ripple of the power distribution in the azimuthal direction (see Eq. (21)).

hod: The lead of the helicoidal wire spacing divided the diameter of the fuel rod.

$\mathrm{dcd}(\mathrm{cm})$ : Thickness of the $\mathrm{B}_{4} \mathrm{C}$ control sheet.

delpr(Pa): Maximum pressure drop for the coolant, this value is used to select a design.

fastf $\left(1 / \mathrm{cm}^{2}\right)$ : Maximum fast tluence at cargo hay.

dose(rad): Maximum gamma dose at cargo bay.

zload(m): Distance of cargo hay to hase of reactor.

rload(m): Radius of cargo bay.

$R(\mathrm{~cm})$ : Radius of core if ishape $=2$.

$\mathrm{H}(\mathrm{cm})$ : Length of core if ishape $=3$.

The input file for the sample case follows:

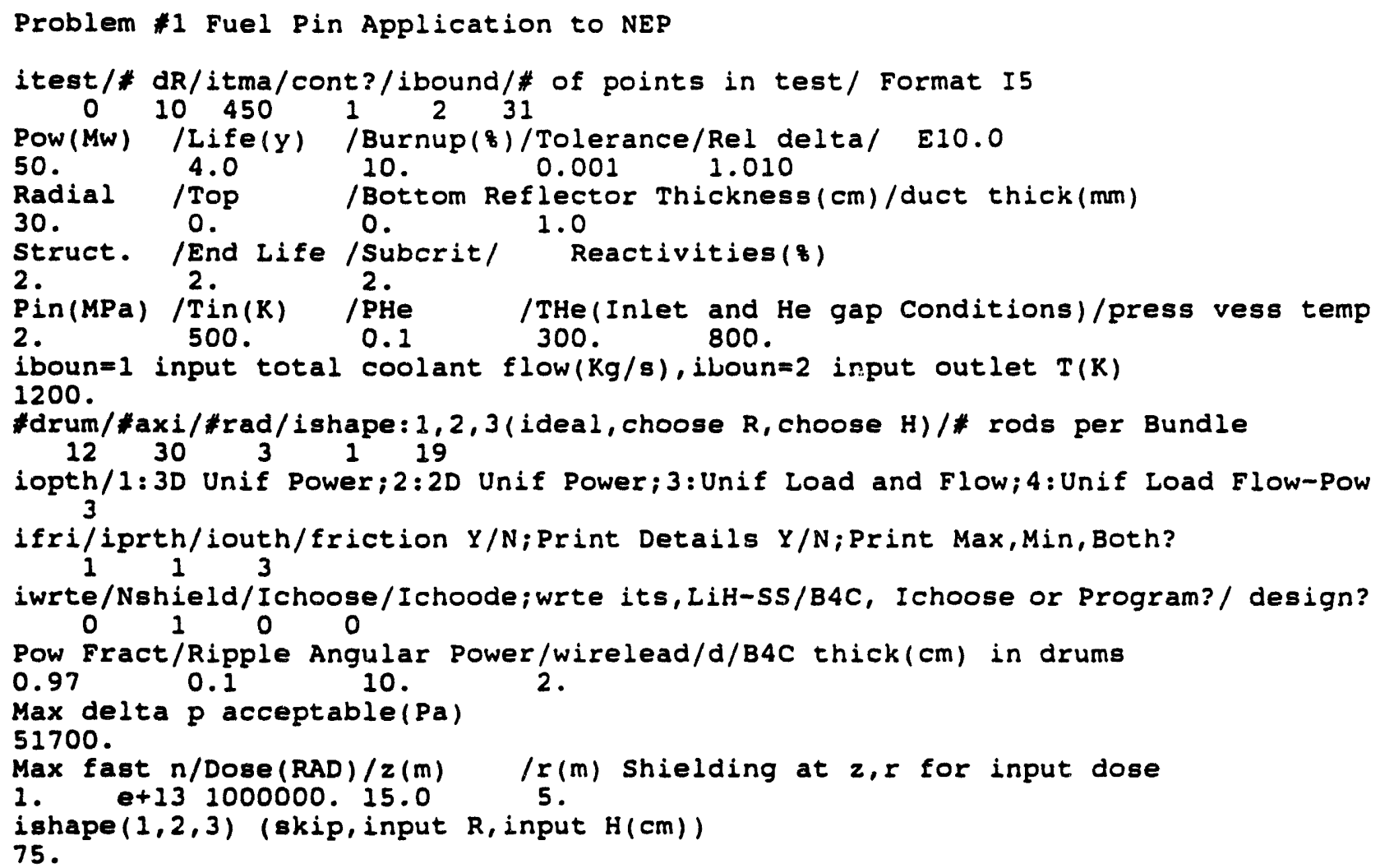




\subsection{OUTPUT FROM NEPPIN}

The output from running the code with the input file of the previous section follow:

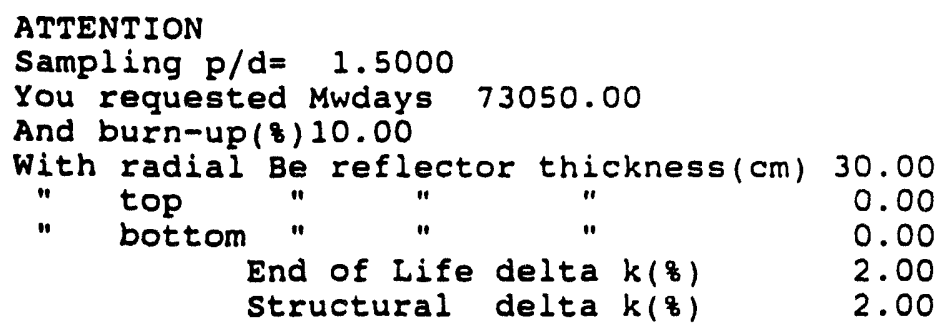

Trying to satisfy reactivity balance, it happens your core

is too large(and it would produce more energy you need).

You might:

1) Reduce the input burn-up, and/or

2) Increase the reflector thickness, and/or

3) Reduce delta $k$ at end of life, and/or

4) Reduce delta k structural

Program will change burn-up for this p/d design and choose ishape $=1$

$\begin{array}{rr}\text { BU } & \text { Mwday } \\ 10.00 & 77233.00 \\ 9.00 & 66788.17\end{array}$

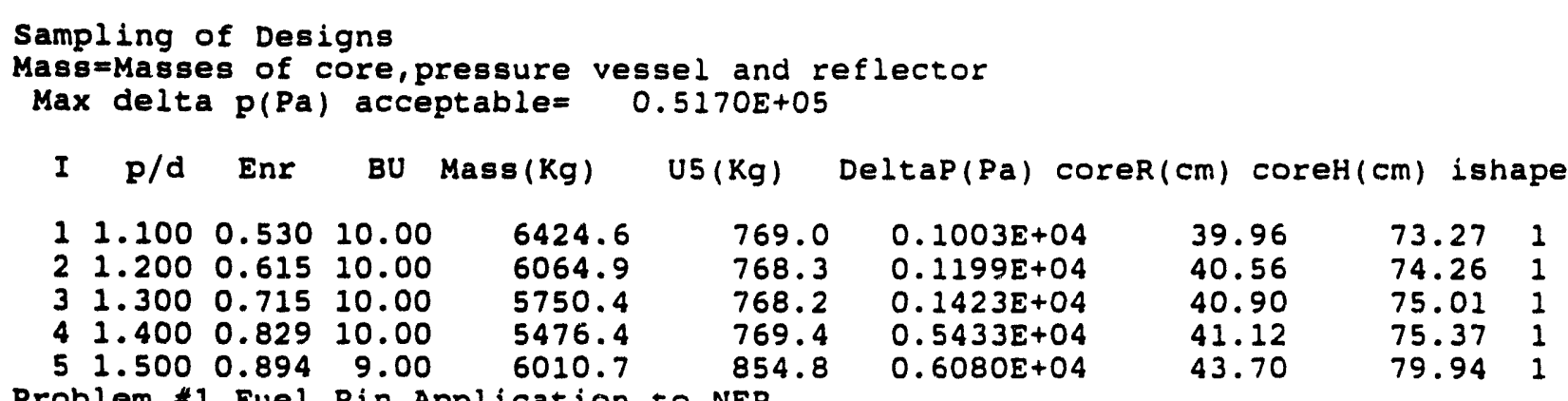

Problem \#1 Fuel Pin Application to NEP

\section{INPUT TO THE PROGRAM}

Power(Mw) $\quad 50.00$ Core life 4.000 years

Energy Released(Mwday) 73050.00 Burn-up(\&) 10.00

Be reflector, radial thickness $(\mathrm{cm}) 30.00$

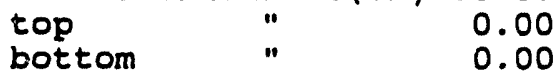

The following design can produce that energy within the relative numerical precision of $0.1000 E-02$

Minimum Core Volume Option Chosen: $R / H=0.541315$

And it has the following reactivity balance

Delta $k$ required(8) 13.99 with components

Fission Products(8) 8.23

Pressure Vessel(8) 1.76

structural(z) 2.00

Page 8-15 
End of Life( 8 ) 2.00

keff at BOL(No structural mat) 1.1399 Components

Bare Reactor 0.9941

Reflector 0.1458

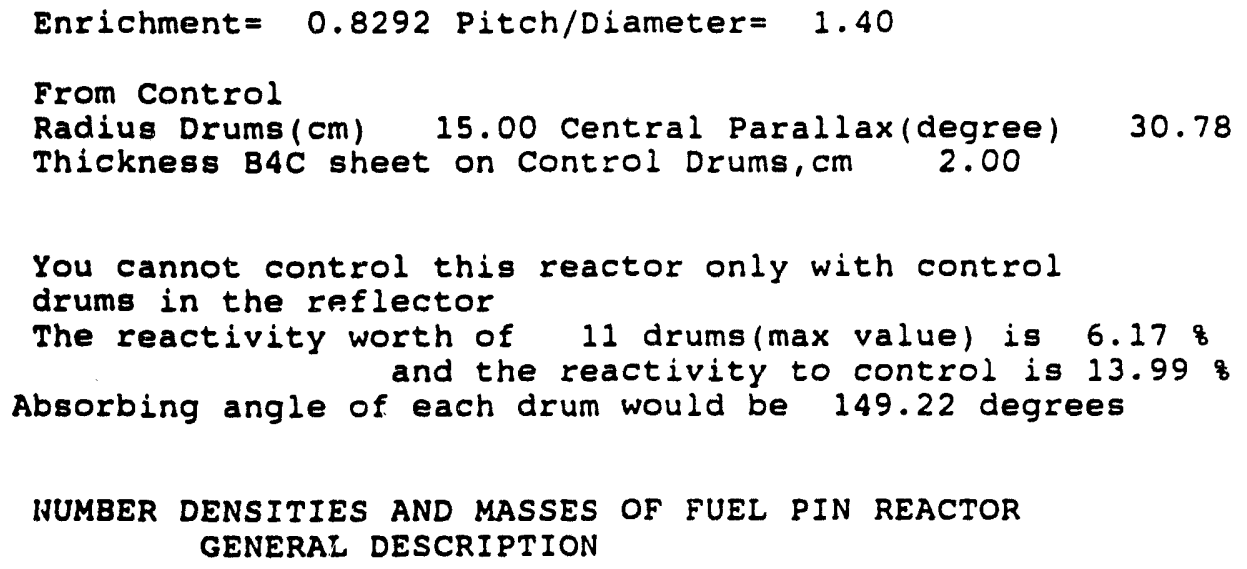




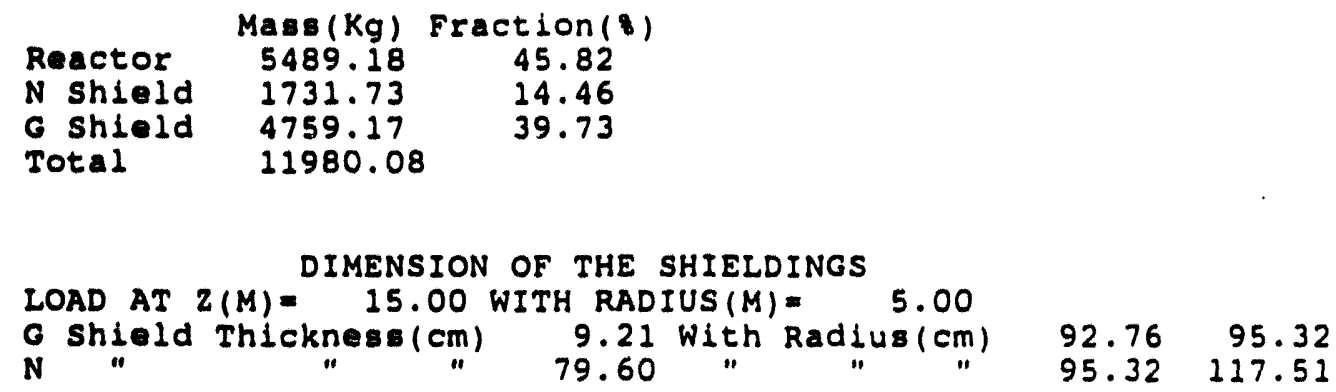

EVALUATION OF PRESSURE VESSEL

Evaluation at $T(K)=800.00$ PRESS (MPa) $=2.00$ and Full Power Life(y) 4.00

Pressure Vessel Thickness $(\mathrm{cm}) \quad 0.40$

\section{DETAILS OF THE SHIELDINGS}

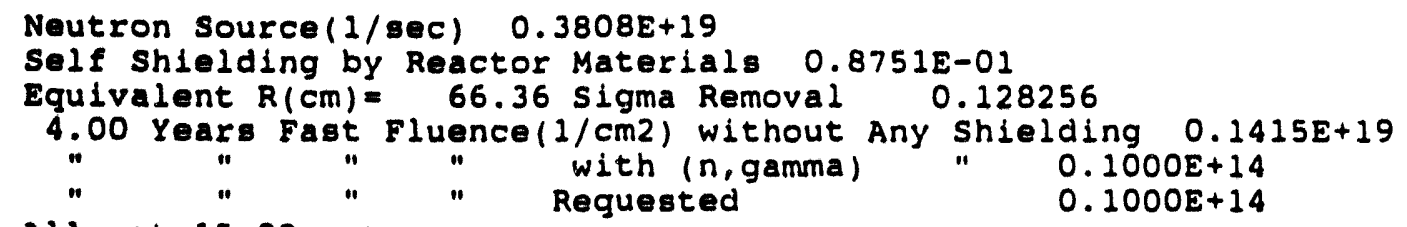

All at 15.00 meters

Neutron Shielding Thickness(cm) 79.60

Neutron Shielding Thickness if no $w$ is present, $\mathrm{cm} \quad 94.05$

NEUTRON SHIELDING: LLH-Stainless Steel Matrix

TOTAL PRODUCTION OF GAMMA RAYS(1/8EC)

$\begin{array}{rrrrrrr}\text { GROUP } 1 & 2 & 3 & 4 & 5 & 7 \\ 0-1 \mathrm{MeV} & 1-2 & 2-3 & 3-5 & 5-7 & 7-9 & >9\end{array}$

$2.132 E+19 \quad 7.140 E+18 \quad 3.132 E+18 \quad 1.474 E+18 \quad 1.815 E+17 \quad 1.493 E+15 \quad 1.097 E+16$

- FRACTION PRODUCED BY U

$\begin{array}{lllllll}89.14 & 75.35 & 62.24 & 32.78 & 35.62 & 0.00 & 99.51\end{array}$

$\begin{array}{ccccccc}\text { Equivalent } Z \text { for Build up factors } & & & & \\ 54.97 & 45.51 & 46.08 & 51.17 & 55.29 & 58.22 & 60.50\end{array}$

Gamma Self shielding by Core, Reflector and $P V$

$\begin{array}{lllllll}0.040546 & 0.098502 & 0.095939 & 0.101189 & 0.097540 & 0.093919 & 0.089193\end{array}$

The same but without Buildup factor

$\begin{array}{llllllll}0.022162 & 0.050059 & 0.057265 & 0.070490 & 0.074171 & 0.074878 & 0.073508\end{array}$

Integrated Dose (Rad) without any shielding( $n$ or gamma)

$1.133 E+092.262 E+091.266 E+098.799 E+08 \quad 1.474 E+08 \quad 1.471 E+06 \quad 1.154 E+07$

Integrated Dose with $w$ and Neutrong Shieldings

1.837E-02 8.058E+04 2.003E+05 $6.097 E+05 \quad 1.045 E+05 \quad 6.534 E+02 \quad 3.629 E+03$

Integrated Dose without Any Shielding, Total(Rad) $0.5701 \mathrm{E}+10$

Integrated dose with Shieldings(W and Neutrons), Total (Rads) $0.10 E+07$

Tungsten Thickness $(\mathrm{cm})$

Tungsten Thickness $(\mathrm{cm}) \quad 11.95$ if no Neutron shield Present

Requested Dose(Rad) 1000000.00 at 15.00 meters 
THERMALHYDRAULICS CALCULATIONS

Average Temperature of the Coolant at Core Exit(K) 1200.00

BOUNDARY CONDITION: OUTLET TEMPERATURE

****PROFILE OF CHANNEL WITH MAXIMUM POWER DENSITY****

Problem \#1 Fuel Pin Application to NEP

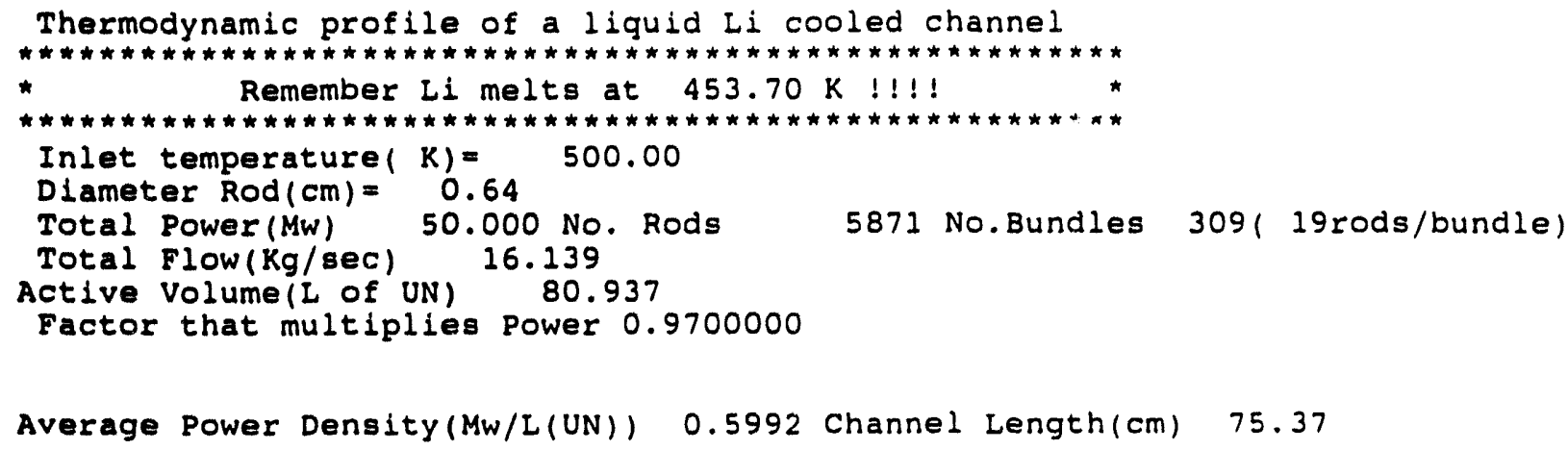

FLOW CONDITIONS:

Pltch/drod 1.400 Lead wire spacer/drod 10.000

Cell Type (per bundle)

12

3 Bundle

Number of Cells

Flow(g/s) Total

Flow Area $(*)$

Flow Area (cm2), per cell

Flow Area (cm2), per rod

Wet Perimeter $(\mathrm{cm})$ Total

Effective Diameter $(\mathrm{cm})$

24
24.2455
0.5126
0.1782
0.3564
1.1394
0.6256

12
23.2914
0.9849
0.3424
0.6848
2.1024
0.6514

4.6917

0.3968

52.2286

0.1379

0.5874

0.8276

1.1394

1.0002

0.4849
58.5741

(*) $1,2,3$ in units of cell 1 area

Parameters of the bundle for friction correlations: $X=0.995 \mathrm{Del} / \mathrm{DT}=0.994$

Boundary Condition: Fix outlet flow temperature 1200.00

Cosine Axial Power Distribution=roav*Pfact*Cos ( $\mathrm{Pi} * \mathrm{Z} / \mathrm{H}$ )

Pfact (Axial*Radial*Azim) $=2.3752$ Extrapolated Delta(cm)

Uniform Coolant Flow Assumed

AVERAGE CONDITIONS IN THE BUNDIE

\begin{tabular}{|c|c|c|c|c|c|c|c|}
\hline \multicolumn{2}{|c|}{$\hbar \star \hbar \hbar * \hbar \hbar \hbar \hbar \hbar \star$} & \multicolumn{2}{|c|}{ Inlet Press(MPa) } & 2.000000 & \multicolumn{3}{|c|}{ 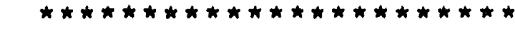 } \\
\hline $\begin{array}{c}\text { Node } \\
1 \\
2 \\
3 \\
4 \\
5 \\
6\end{array}$ & $\begin{array}{c}z(\mathrm{~cm}) \\
0.00 \\
2.51 \\
5.02 \\
7.54 \\
10.05 \\
12.56\end{array}$ & $\begin{array}{c}P O(w / \mathrm{cm}) \\
47.64 \\
71.06 \\
93.88 \\
115.90 \\
136.93 \\
156.79\end{array}$ & $\begin{array}{c}\text { Pr (KPa) } \\
2000.00 \\
2000.00 \\
1999.99 \\
1999.99 \\
1999.99 \\
1999.98\end{array}$ & $\begin{array}{l}T(K) \\
500.001 \\
511.821 \\
528.401 \\
549.711 \\
575.681 \\
606.151\end{array}$ & $\begin{array}{c}\text { (Tsat) } \\
2129.52) \\
2129.52) \\
2129.52) \\
2129.52) \\
2129.52) \\
2129.51)\end{array}$ & $\begin{array}{c}V(\mathrm{~m} / \mathrm{s}) \\
0.11 \\
0.11 \\
0.11 \\
0.11 \\
0.11 \\
0.11\end{array}$ & $\begin{array}{l}\text { Re } \\
274 . \\
296 . \\
328 . \\
371 . \\
426 . \\
493 .\end{array}$ \\
\hline
\end{tabular}




$\begin{array}{rrrrrrrr}7 & 15.07 & 175.31 & 1999.98 & 640.90(2129.51) & 0.11 & 572 . \\ 8 & 17.59 & 192.33 & 1999.98 & 679.65(2129.51) & 0.11 & 664 . \\ 9 & 20.10 & 207.71 & 1999.97 & 722.07(2129.51) & 0.12 & 767 . \\ 10 & 22.61 & 221.31 & 1999.97 & 767.79(2129.51) & 0.12 & 880 . \\ 11 & 25.12 & 233.03 & 1999.97 & 816.40(2129.51) & 0.12 & 1002 . \\ 12 & 27.63 & 242.75 & 1999.97 & 867.47(2129.51) & 0.12 & 1130 . \\ 13 & 30.15 & 250.40 & 1999.96 & 920.54(2129.51) & 0.12 & 1263 . \\ 14 & 32.66 & 255.91 & 1999.96 & 975.13(2129.51) & 0.12 & 1399 . \\ 15 & 35.17 & 259.24 & 1999.95 & 1030.77(2129.51) & 0.12 & 1534 . \\ 16 & 37.68 & 260.35 & 1999.95 & 1086.96(2129.51) & 0.13 & 1668 . \\ 17 & 40.19 & 259.24 & 1999.95 & 1143.21(2129.51) & 0.13 & 1799 . \\ 18 & 42.71 & 255.91 & 1999.94 & 1199.03(2129.51) & 0.13 & 1926 . \\ 19 & 45.22 & 250.40 & 1999.94 & 1253.93(2129.51) & 0.13 & 2048 . \\ 20 & 47.73 & 242.75 & 1999.94 & 1307.44(2129.51) & 0.13 & 2162 . \\ 21 & 50.24 & 233.03 & 1999.93 & 1359.09(2129.51) & 0.13 & 2270 . \\ 22 & 52.76 & 221.31 & 1999.93 & 1408.42(2129.51) & 0.13 & 2370 . \\ 23 & 55.27 & 207.71 & 1999.93 & 1455.03(2129.51) & 0.14 & 2462 . \\ 24 & 57.78 & 192.33 & 1999.92 & 1498.50(2129.51) & 0.14 & 2545 . \\ 25 & 60.29 & 175.31 & 1999.92 & 1538.46(2129.51) & 0.14 & 2620 . \\ 26 & 62.80 & 156.79 & 1999.91 & 1574.56(2129.51) & 0.14 & 2686 . \\ 27 & 65.32 & 136.93 & 1999.91 & 1606.49(2129.51) & 0.14 & 2743 . \\ 28 & 67.83 & 115.90 & 1999.91 & 1633.99(2129.51) & 0.14 & 2792 . \\ 29 & 70.34 & 93.88 & 1999.90 & 1656.80(2129.51) & 0.14 & 2832 . \\ 30 & 72.85 & 71.06 & 1999.90 & 1674.74(2129.50) & 0.14 & 2862 . \\ 31 & 75.37 & 47.64 & 1999.90 & 1687.65(2129.50) & 0.14 & 2884 .\end{array}$

Thermodynamics Conditions of Cells

$\begin{array}{rrrrr}\text { Cell Type } & 2 \text { Node } & \text { Max }(T-<T>) & T & \text { Saturation } P(k P a) \\ 1 & 31 & 434.21 & 2121.87 & 1937.51 \\ 2 & 31 & -351.02 & 1336.63 & 10.78 \\ 3 & 31 & -497.75 & 1189.91 & 1.91\end{array}$

Delta pressure(KPa) because TI>T(average) 1738.05

$\left\langle c>\right.$ in $f=c / R e^{\star *} 0.25$ is 0.25944

From the Coolant Channel to fuel pin temperature
Rod Diameter(mm): 6.4000
Clad+Liner Thickness ("): 0.7620
He gap $\quad$ ("): 0.0250
Fuel Pin Diameter. ("): 4.8260

CONDITIONS FOR MAX HEATED ROD OF THE BUNDLE(TYPE 1 cell)

Node

$2(\mathrm{~cm}) J(\mathrm{Kw} / \mathrm{cm} 2)$

Twall (K)

$\operatorname{Tclad}(K)$

TcladAv

Tfuel (K)

TfuelAv

$\begin{array}{rrrrrrrr}1 & 0.00 & 0.02369 & 502.51 & 506.13 & 504.15 & 549.07 & 541.17 \\ 2 & 2.51 & 0.03534 & 519.82 & 525.22 & 522.27 & 588.24 & 576.46 \\ 3 & 5.02 & 0.04669 & 543.62 & 550.75 & 546.87 & 632.31 & 616.75 \\ 4 & 7.54 & 0.05764 & 573.89 & 582.69 & 577.89 & 680.96 & 661.75 \\ 5 & 10.05 & 0.06810 & 610.48 & 620.88 & 615.22 & 733.91 & 711.21 \\ 6 & 12.56 & 0.07798 & 653.17 & 665.08 & 658.59 & 790.85 & 764.86 \\ 7 & 15.07 & 0.08719 & 701.62 & 714.94 & 707.68 & 851.49 & 822.42 \\ 8 & 17.59 & 0.09566 & 755.42 & 770.03 & 762.06 & 915.47 & 883.59 \\ 9 & 20.10 & 0.10330 & 814.09 & 829.86 & 821.27 & 982.43 & 948.00 \\ 10 & 22.61 & 0.11007 & 877.11 & 893.92 & 884.76 & 1051.95 & 1015.26 \\ 11 & 25.12 & 0.11590 & 943.92 & 961.62 & 951.97 & 1123.57 & 1084.94 \\ 12 & 27.63 & 0.12073 & 1013.93 & 1032.36 & 1022.32 & 1196.79 & 1156.55 \\ 13 & 30.15 & 0.12454 & 1086.52 & 1105.54 & 1095.17 & 1271.09 & 1229.57 \\ 14 & 32.66 & 0.12728 & 1161.06 & 1180.49 & 1169.90 & 1345.90 & 1303.47 \\ 15 & 35.17 & 0.12893 & 1236.89 & 1256.58 & 1245.85 & 1420.64 & 1377.66 \\ 16 & 37.68 & 0.12949 & 1313.37 & 1333.14 & 1322.36 & 1494.71 & 1451.55 \\ 17 & 40.19 & 0.12893 & 1389.82 & 1409.51 & 1398.78 & 1567.53 & 1524.55 \\ 18 & 42.71 & 0.12728 & 1465.60 & 1485.03 & 1474.44 & 1638.47 & 1596.04\end{array}$

Page 8-20 


$\begin{array}{llllllll}19 & 45.22 & 0.12454 & 1540.05 & 1559.06 & 1548.70 & 1706.94 & 1665.43 \\ 20 & 47.73 & 0.12073 & 1612.52 & 1630.95 & 1620.91 & 1772.36 & 1732.12 \\ 21 & 50.24 & 0.11590 & 1682.40 & 1700.10 & 1690.45 & 1834.16 & 1795.53 \\ 22 & 52.76 & 0.11007 & 1749.08 & 1765.89 & 1756.73 & 1891.80 & 1855.11 \\ 23 & 55.27 & 0.10330 & 1812.00 & 1827.77 & 1819.17 & 1944.76 & 1910.33 \\ 24 & 57.78 & 0.09566 & 1870.60 & 1885.21 & 1877.24 & 1992.58 & 1960.69 \\ 25 & 60.29 & 0.08719 & 1924.39 & 1937.70 & 1930.44 & 2034.81 & 2005.75 \\ 26 & 62.80 & 0.07798 & 1972.89 & 1984.80 & 1978.31 & 2071.07 & 2045.08 \\ 27 & 65.32 & 0.06810 & 2015.70 & 2026.10 & 2020.43 & 2101.02 & 2078.32 \\ 28 & 67.83 & 0.05764 & 2052.44 & 2061.25 & 2056.45 & 2124.36 & 2105.14 \\ 29 & 70.34 & 0.04669 & 2082.80 & 2089.93 & 2086.04 & 2140.86 & 2125.29 \\ 30 & 72.85 & 0.03534 & 2106.50 & 2111.90 & 2108.96 & 2150.34 & 2138.56 \\ 31 & 75.37 & 0.02369 & 2123.35 & 2126.97 & 2124.99 & 2152.68 & 2144.79\end{array}$

Axial Average Conditions in the Channel

Coolant Pressure (MPa) 2.000 Temperature(K) 1089.432

Wall Temperature (K) 1313.155 Heat Transfer (w/cm2K) $\quad 12.70$

Clad Temperature (K) 1319.526

Fuel Temperature (K) 1416.165

HEAT BALANCE FOR THE BUNDLE

dQ: heat to coolant (joule/kg) $0.50566 \mathrm{E}+07$
dH: change of enthalpy (joule/kg) $0.50566 \mathrm{E}+07$
dKin change kinetic energy (joule/kg) $0.43164 \mathrm{E}-02$
relative dKin/dQ 0.000000
Balance (dH+dKin)/dQ 0.9999961

***PROFILE OF CHANNEL WITH MINIMUM POWER DENSITY***

Problem \# Fuel Pin Application to NEP

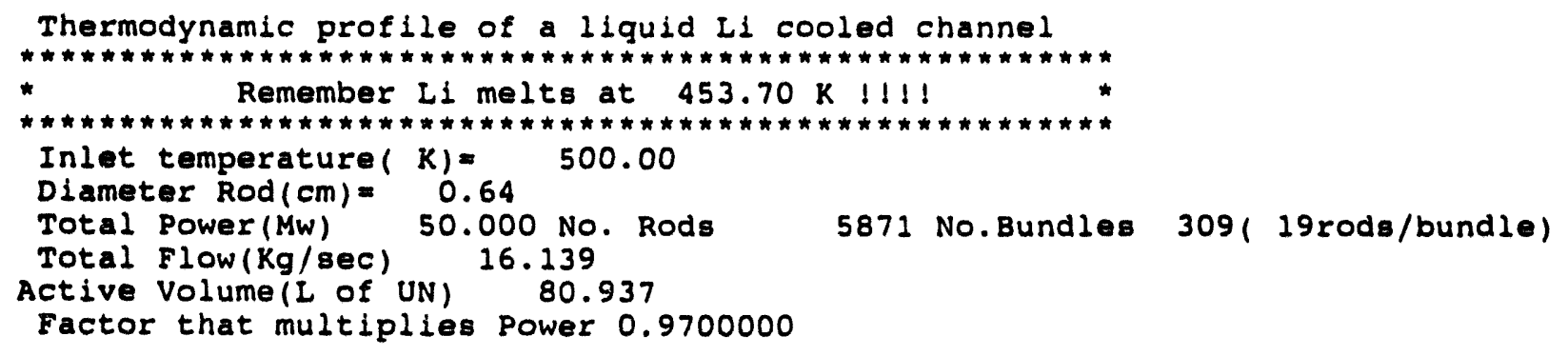

Average Power Density (MW/L(UN)) 0.5992 Channel Length $(\mathrm{cm}) \quad 75.37$

FLOW CONDITIONS :

Pitch/drod 1.400 Lead wire spacer/drod 10.000

$\begin{array}{llllll}\text { Cell Type (per bundle) } & 1 & 2 & 3 & \text { Bundle }\end{array}$

$\begin{array}{lrrrr}\text { Number of Cells } & 24 & 12 & 6 & \\ \text { Flow(g/s) Total } & 24.2455 & 23.2914 & 4.6917 & 52.2286 \\ \text { Flow Area (*) } & 0.5126 & 0.9849 & 0.3968 & 0.5874 \\ \text { Flow Area (cm2), per cell } & 0.1782 & 0.3424 & 0.1379 & 9.2130 \\ \text { Flow Area (cm2), per rod } & 0.3564 & 0.6848 & 0.8276 & 0.4849 \\ \text { Wet Perimeter(cm) Total } & 1.1394 & 2.1024 & 1.0002 & 58.5741 \\ \text { Effective Diameter (cm) } & 0.6256 & 0.6514 & 0.5516 & 0.6291\end{array}$

(*) $1,2,3$ in units of cell 1 area

Parameters of the bundle for friction correlations: $X=0.995$ Del $/ D T=0.994$ 
Boundary Condition: F1x outlet flow temperature 2152.68

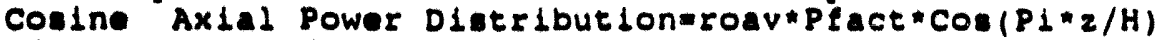
Pfact (AxLal Radial Az $1 \mathrm{~m})=0.5662$ Extrapolated Delta(cm) Uniform Coolant Flow Aseumed

AVERACE CONDITIONS IN THE BUNDLE

\begin{tabular}{|c|c|c|c|c|c|c|c|}
\hline \multicolumn{2}{|c|}{ 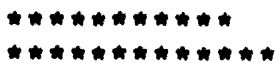 } & \multicolumn{2}{|c|}{ Inlet Press (MPa) } & 2.000000 & \multicolumn{3}{|c|}{ 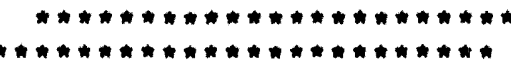 } \\
\hline $\begin{array}{c}\text { Node } \\
1 \\
2 \\
3 \\
4 \\
5 \\
6 \\
7 \\
8 \\
9 \\
10 \\
11 \\
12 \\
13 \\
14 \\
15 \\
16 \\
17 \\
18 \\
19 \\
20 \\
21 \\
22 \\
23 \\
24 \\
25 \\
26 \\
27 \\
28 \\
29 \\
30 \\
31\end{array}$ & $\begin{array}{l}2(\mathrm{~cm}) \\
0.00 \\
2.51 \\
5.02 \\
7.54 \\
10.05 \\
12.56 \\
15.07 \\
17.59 \\
20.10 \\
22.61 \\
25.12 \\
27.63 \\
30.15 \\
32.66 \\
35.17 \\
37.68 \\
40.19 \\
42.71 \\
45.22 \\
47.73 \\
50.24 \\
52.76 \\
55.27 \\
57.78 \\
60.29 \\
62.80 \\
65.32 \\
67.83 \\
70.34 \\
72.85 \\
75.37\end{array}$ & $\begin{array}{l}P 0(w / c m) \\
11.36 \\
16.94 \\
22.38 \\
27.63 \\
32.64 \\
37.38 \\
41.79 \\
45.85 \\
49.51 \\
52.76 \\
55.55 \\
57.87 \\
59.69 \\
61.01 \\
61.80 \\
62.06 \\
61.80 \\
61.01 \\
59.69 \\
57.87 \\
55.55 \\
52.76 \\
49.51 \\
45.85 \\
41.79 \\
37.38 \\
32.64 \\
27.63 \\
22.38 \\
16.94 \\
11.36\end{array}$ & 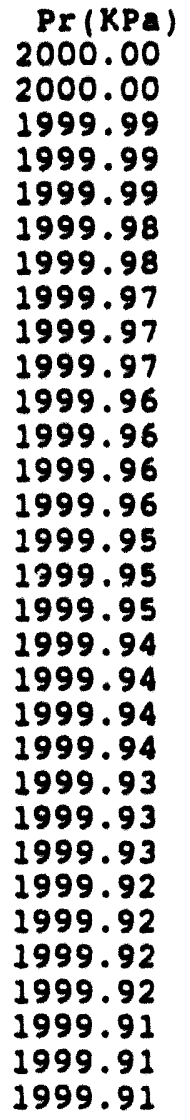 & $\begin{array}{l}T(K) \\
500.001 \\
502.81( \\
506.721 \\
511.711 \\
517.751 \\
524.791 \\
532.791 \\
541.691 \\
551.421 \\
561.891 \\
573.041 \\
584.761 \\
596.961 \\
609.521 \\
622.351 \\
635.331 \\
648.341 \\
661.271 \\
674.011 \\
686.441 \\
698.461 \\
709.951 \\
720.821 \\
730.961 \\
740.291 \\
748.731 \\
756.201 \\
762.631 \\
767.971 \\
772.171 \\
775.191\end{array}$ & $\begin{array}{c}(\text { TaAt) } \\
2129.52) \\
2129.52) \\
2129.52) \\
2129.52) \\
2129.52) \\
2129.51) \\
2129.51) \\
2129.51) \\
2129.51) \\
2129.51) \\
2129.51) \\
2129.51) \\
2129.51) \\
2129.51) \\
2129.51) \\
2129.51) \\
2129.51) \\
2129.51) \\
2129.51) \\
2129.51) \\
2129.51) \\
2129.51) \\
2129.51) \\
2129.511 \\
2129.51) \\
2129.51) \\
2129.51) \\
2129.51) \\
2129.51) \\
2129.51) \\
2129.51)\end{array}$ & $\begin{array}{c}V(\mathrm{~m} / 1) \\
0.11 \\
0.11 \\
0.11 \\
0.11 \\
0.11 \\
0.11 \\
0.11 \\
0.11 \\
0.11 \\
0.11 \\
0.11 \\
0.11 \\
0.11 \\
0.11 \\
0.11 \\
0.11 \\
0.11 \\
0.11 \\
0.11 \\
0.11 \\
0.12 \\
0.12 \\
0.12 \\
0.12 \\
0.12 \\
0.12 \\
0.12 \\
0.12 \\
0.12 \\
0.12 \\
0.12\end{array}$ & 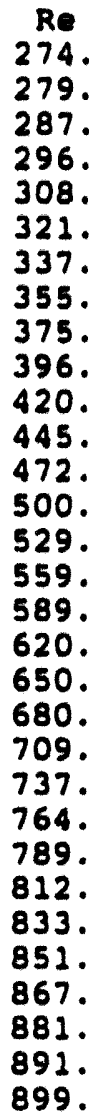 \\
\hline
\end{tabular}

Thermodynamics Conditione of Cells

Cell Type 2 Node $\operatorname{Max}(T-<T>)$

T saturation $P(k P a)$

$\begin{array}{llrll}1 & 31 & 101.98 & 877.17 & 0.01 \\ 2 & 31 & -81.96 & 693.23 & 0.00 \\ 3 & 31 & -116.03 & 659.16 & 0.00\end{array}$

Delta preseure(KPa) because Tl>T(average) 0.01

$\langle c\rangle$ in $f=c / R e * * 0.25$ is 0.19946

From the Coolant Channel to fuel pin temperature

Rod Diameter $(\mathrm{mm}): 6.4000$

CladtLiner Thicknese( "): 0.7620

He gap " ("): 0.0250

Fuel Pin Diameter ("): 4.8260 
CONDITIONS FOR MAX HEATED ROD OF THE BUNDLE(TYPE 1 CE11)

\begin{tabular}{|c|c|c|c|c|c|c|c|}
\hline Node & $2(\mathrm{~cm})$ & $J(\mathrm{Kw} / \mathrm{cm} 2)$ & Twa $11(K)$ & $\operatorname{rclad}(K)$ & TCladAV & rfuel $(K)$ & Tfuelav \\
\hline $\begin{array}{l}1 \\
2 \\
3 \\
4 \\
5 \\
6 \\
7 \\
8 \\
9 \\
10 \\
11 \\
12 \\
13 \\
14 \\
15 \\
16 \\
17 \\
18 \\
19 \\
20 \\
21 \\
22 \\
23 \\
24 \\
25 \\
26 \\
27 \\
28 \\
29 \\
30 \\
31\end{array}$ & $\begin{array}{r}0.00 \\
2.51 \\
5.02 \\
7.54 \\
10.05 \\
12.56 \\
15.07 \\
17.59 \\
20.10 \\
22.61 \\
25.12 \\
27.63 \\
30.15 \\
32.66 \\
35.17 \\
37.68 \\
40.19 \\
42.71 \\
45.22 \\
47.73 \\
50.24 \\
52.76 \\
55.27 \\
57.78 \\
60.29 \\
62.80 \\
65.32 \\
67.83 \\
70.34 \\
72.85 \\
75.37\end{array}$ & $\begin{array}{l}0.00565 \\
0.00843 \\
0.01113 \\
0.01374 \\
0.01623 \\
0.01859 \\
0.02079 \\
0.02280 \\
0.02463 \\
0.02624 \\
0.02763 \\
0.02878 \\
0.02969 \\
0.03034 \\
0.03074 \\
0.03087 \\
0.03074 \\
0.03034 \\
0.02969 \\
0.02878 \\
0.02763 \\
0.02624 \\
0.02463 \\
0.02280 \\
0.02079 \\
0.01859 \\
0.01623 \\
0.01374 \\
0.01113 \\
0.00843 \\
0.00565\end{array}$ & $\begin{array}{l}500.60 \\
504.71 \\
510.32 \\
517.40 \\
525.90 \\
535.77 \\
546.93 \\
559.32 \\
572.82 \\
587.34 \\
602.76 \\
618.94 \\
635.76 \\
653.05 \\
670.68 \\
688.48 \\
706.30 \\
723.99 \\
741.38 \\
758.32 \\
774.67 \\
790.28 \\
805.00 \\
818.72 \\
831.31 \\
842.65 \\
852.66 \\
861.23 \\
868.30 \\
873.80 \\
877.68\end{array}$ & $\begin{array}{l}501.46 \\
506.00 \\
512.02 \\
519.50 \\
528.38 \\
538.61 \\
550.11 \\
562.80 \\
576.58 \\
591.35 \\
606.98 \\
623.34 \\
640.29 \\
657.68 \\
675.37 \\
693.19 \\
710.99 \\
728.62 \\
745.91 \\
762.72 \\
778.89 \\
794.28 \\
808.76 \\
822.20 \\
834.48 \\
845.49 \\
855.13 \\
863.33 \\
870.00 \\
875.09 \\
878.55\end{array}$ & $\begin{array}{l}500.99 \\
505.30 \\
511.10 \\
510.35 \\
527.03 \\
537.06 \\
548.38 \\
560.90 \\
574.53 \\
589.17 \\
604.68 \\
620.94 \\
637.82 \\
655.16 \\
672.81 \\
690.62 \\
708.44 \\
726.09 \\
743.44 \\
760.32 \\
776.59 \\
792.10 \\
806.71 \\
820.30 \\
832.75 \\
843.94 \\
853.78 \\
862.18 \\
869.07 \\
874.39 \\
878.08\end{array}$ & $\begin{array}{l}512.74 \\
521.27 \\
532.10 \\
544.12 \\
557.25 \\
571.39 \\
586.43 \\
602.26 \\
618.76 \\
635.80 \\
653.27 \\
671.03 \\
688.93 \\
706.85 \\
724.63 \\
742.14 \\
759.24 \\
775.78 \\
791.63 \\
806.65 \\
820.71 \\
833.70 \\
845.50 \\
856.00 \\
865.11 \\
872.75 \\
878.84 \\
883.32 \\
886.14 \\
887.28 \\
886.71\end{array}$ & $\begin{array}{l}509.86 \\
518.46 \\
528.39 \\
539.54 \\
551.84 \\
565.19 \\
579.50 \\
594.66 \\
610.55 \\
627.06 \\
644.06 \\
661.43 \\
679.04 \\
696.73 \\
714.39 \\
731.85 \\
748.99 \\
765.67 \\
781.73 \\
797.05 \\
811.50 \\
824.95 \\
837.29 \\
848.40 \\
858.18 \\
866.55 \\
873.43 \\
878.74 \\
882.43 \\
884.47 \\
884.83\end{array}$ \\
\hline
\end{tabular}

Axial Average Conditions in the Channel

Coolant Presaure(MPa) 2.000 Temperature(K) 636.312

Wall Tomperature(K) 688.931 Heat Transfer (w/cm2K) $\quad 10.20$

clad Tomperature (K) 690.450

Fuel Temperature(K) 719.981

HEAT BALANCE FOR THE BUNDLE

dQ: heat to coolant (joule/kg) $0.12054 E+07$

dH: change of enthalpy (joule/kg) $0.12054 \mathrm{E}+07$

dKIn change kinetic energy(joule/kg) $0.72267 \mathrm{E}-03$

relative $\mathrm{dK} i \mathrm{n} / \mathrm{dQ} 0.000000$

Balance $(\mathrm{dH}+\mathrm{dK} / \mathrm{n}) / \mathrm{dQ} \quad 0.9999923$ 


\subsection{References}

1. N. M. Greene. W. E. Ford III. L. M. Petrie. J. W. Arwoud. "AMPX-77: A Modular Code System for Generating Coupled Multigroun Neutron-Gamına Cross Sections Libraries from ENDF/B -IV and/or ENDF/B.V." ORNL/CSD/TM-283 (Oituber 1992).

2. W. E. Ford III, et. al., "ANSL-V: ENDF/B-V Based Multigroup Cross-Section Libraries for Advanced Neutron Source (ANS) Reactor Studies," ORNL-6618 (Septemher 1990).

3. John R. Lamarsh, "Introduction to Nuclear Reactur Theory." Addison-Wesley Publ. Co. (1966).

4. J. C. Moyers and J. P. Nichols. "ALKASYS, A Cumputer Program fur Studies of Rankine-Cyde Space Nuclear Power Systems." ORNL/TM-10427 (Septemher 1987).

5. "Reactor Physics Constants," ANL-5800 Second Edition (July 1963).

6. G. Melese and R. Katz "Thermal and Flow Design of Helium - Cooled Reactors." American Nuclear Society (1984).

7. Y. S. Tang, R. D. Coffield, and R. A. Markley. "Thermal Analysis of Liquid-Metal Fast Breeder Reactor," American Nuclear Society (1978).

8. D. W. Jeppson, et. al., "Lithium Literature Review: Lithium's Properties and Interactions," HEDL-TME 78-15 UC-20. Hanford Engineering Development Lahoratory (April 1978).

9. M. M. El-Wakil. "Nuclear Power Engineering." MiGraw-Hill Book Co. (1962).

10. R. W. Bussard and R. D. DeLauer. "Fundamental of Nuclear Flight." McGraw-Hill Book Co. (1965).

11. "CRC Handhook of Chemistry and Physics," 48th Edition (1967-1968).

12. "Steam, its Generation and Use." Bahcock and Wilcox. 38th Edition (1972). 


\section{APPENDIX A. LISTING OF CODE NEPNERYA}

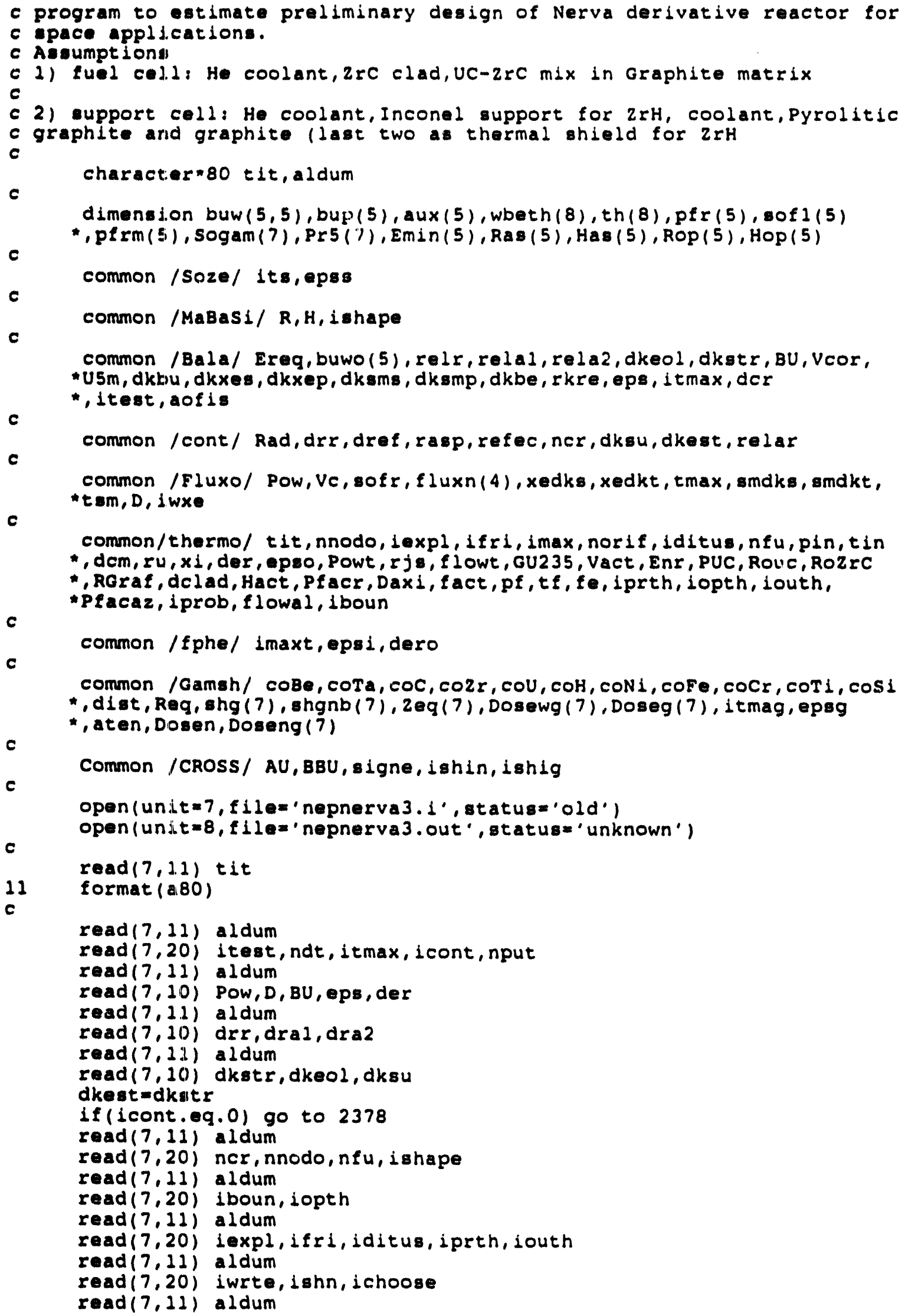

Page A-1 


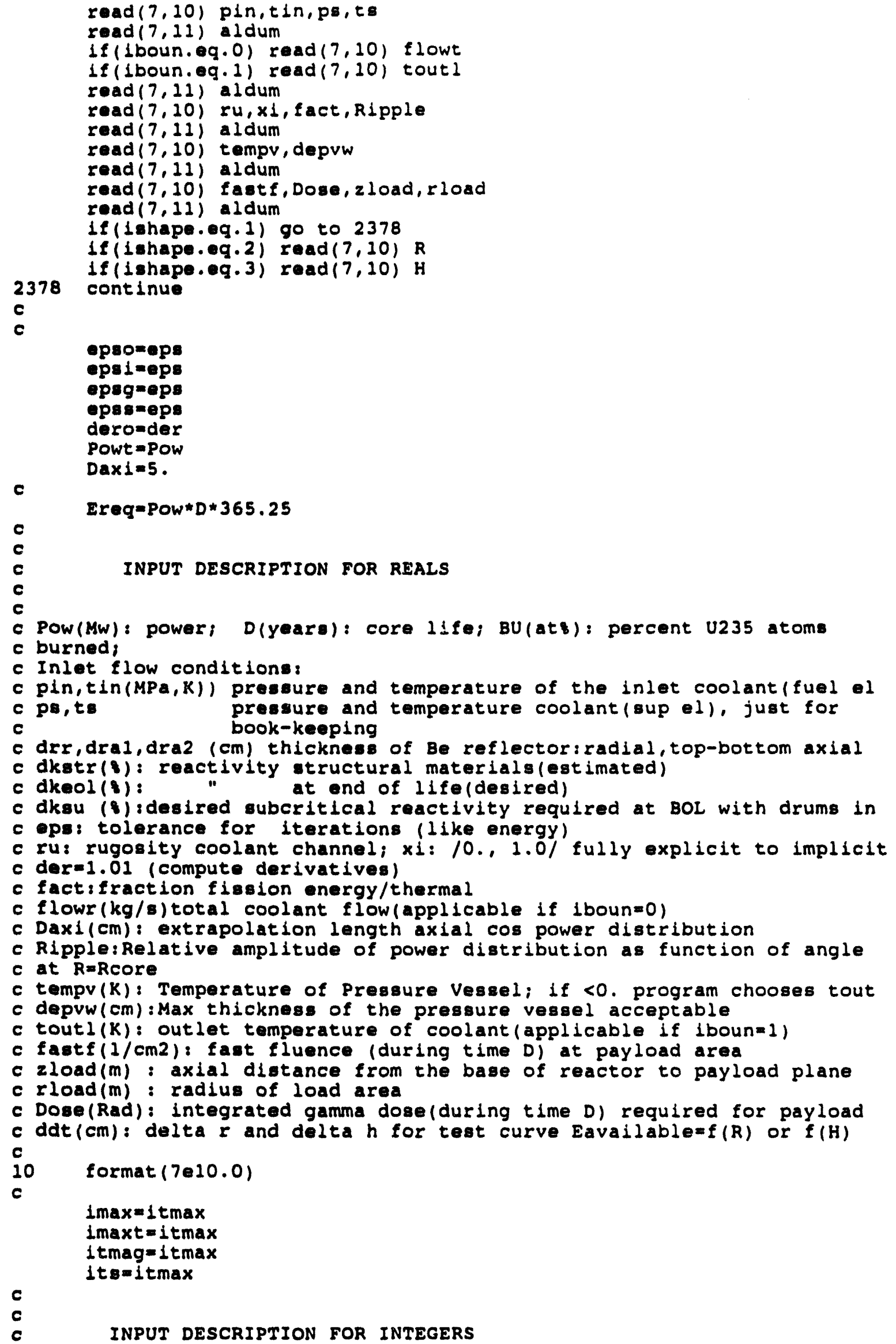




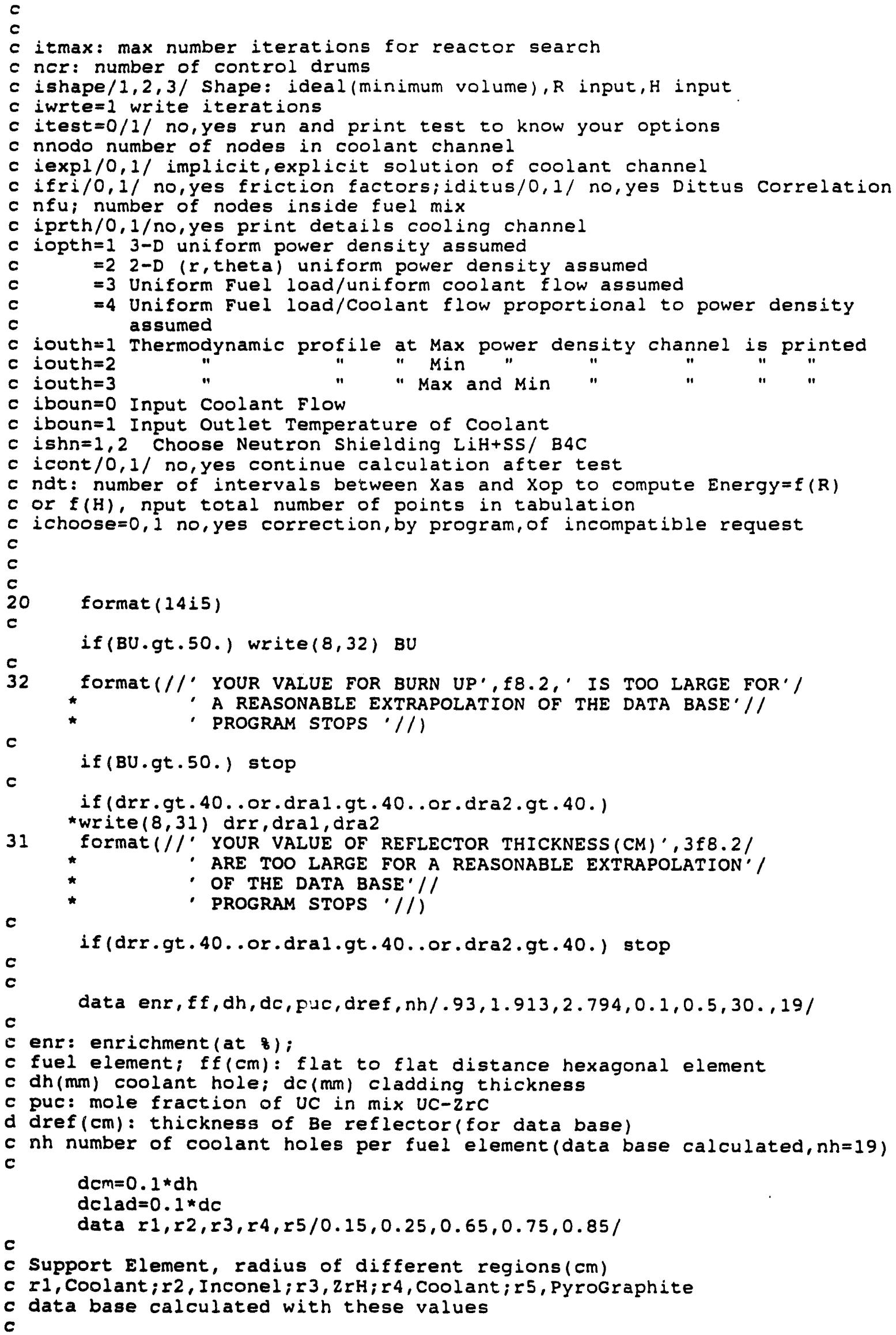




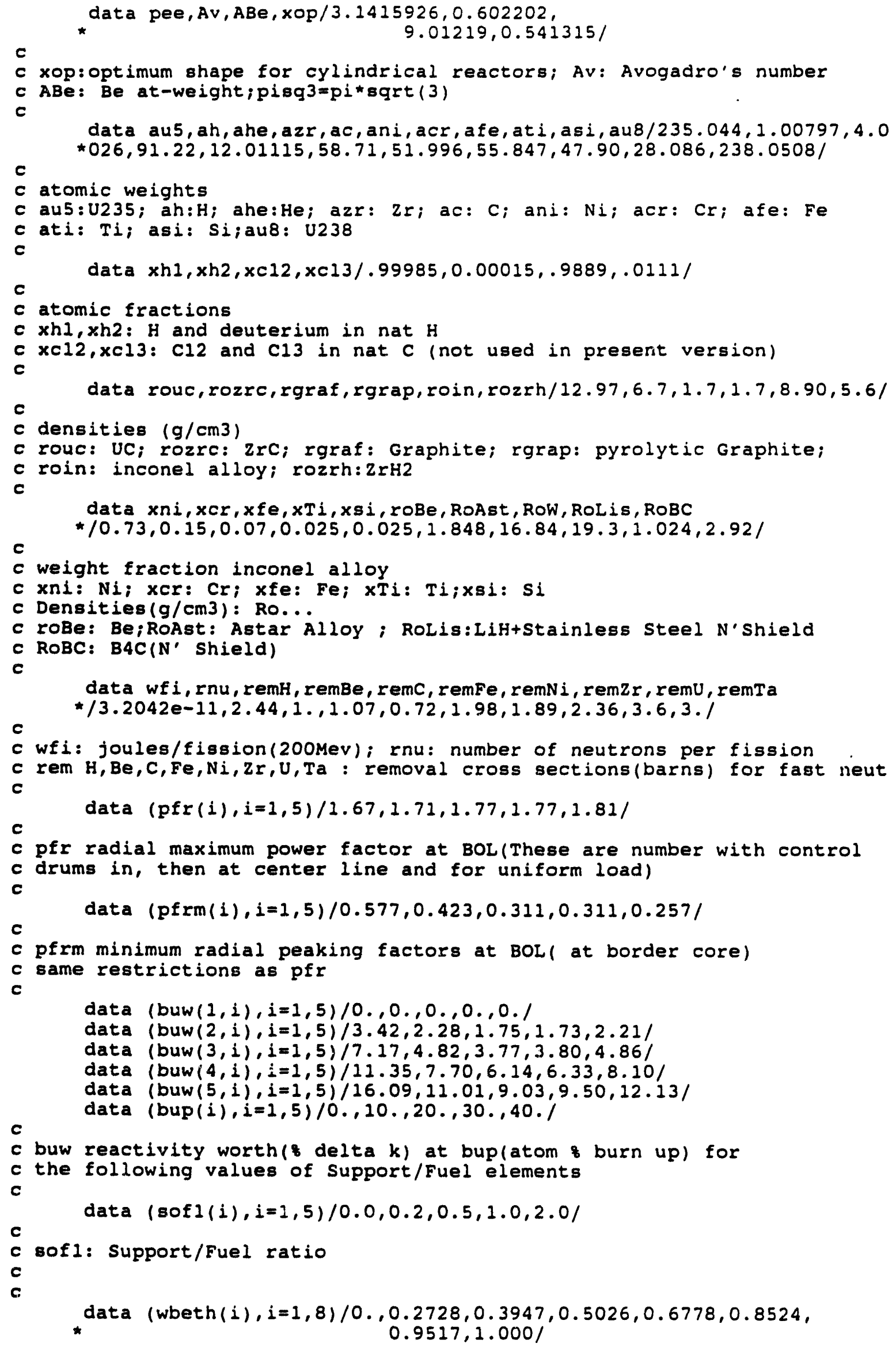




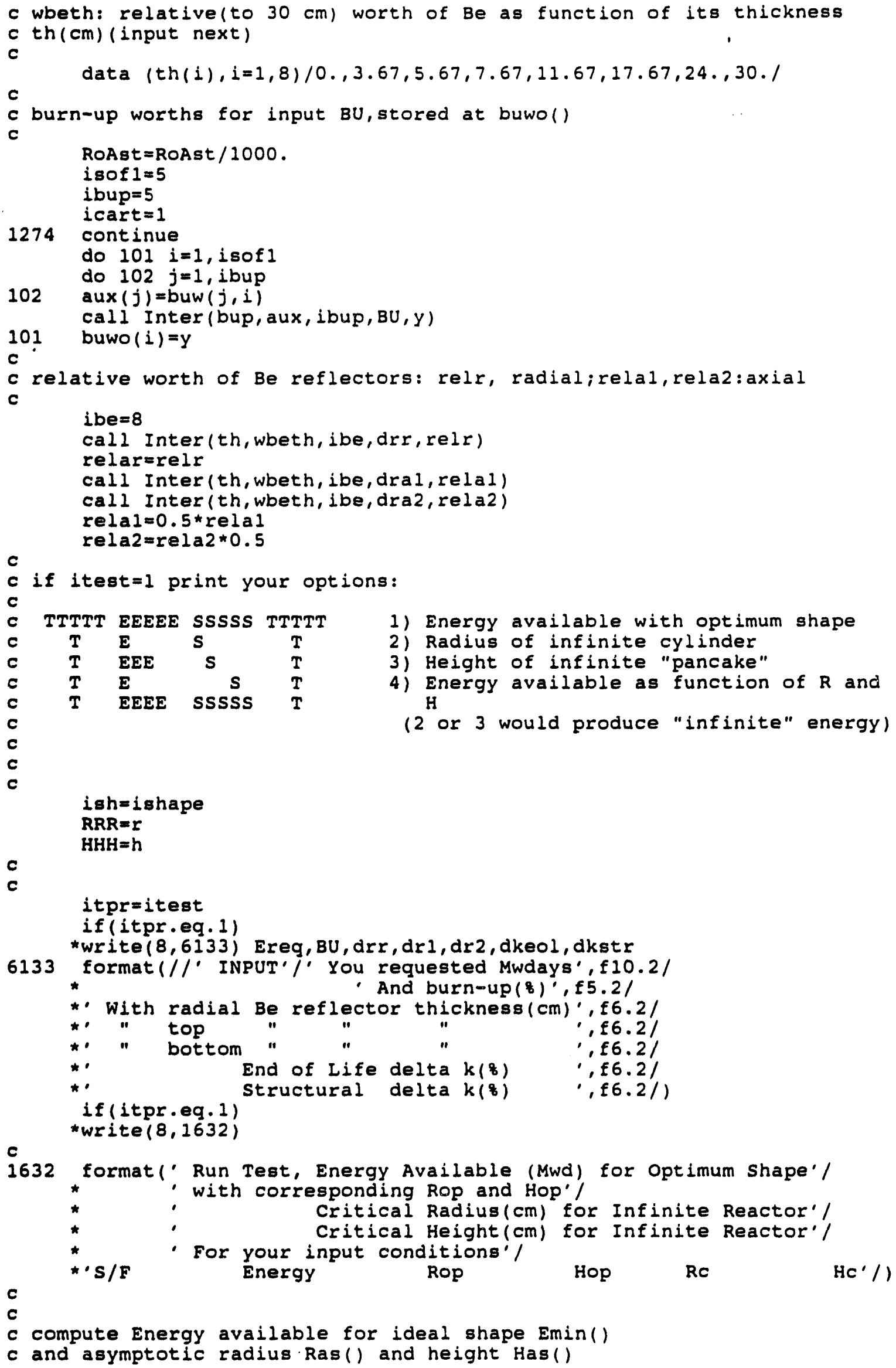


c

itest $=1$

do 1633 is $=1, i s o f 1$

ishape $=1$

sofac $=80 f 1$ (is)

Ene $=$ Balance ( $i b u$, sofac)

$\operatorname{Emin}($ is $)=$ Ene

$\operatorname{ROp}(i 8)=R$

HOp (is) $=H$

Ropo $=\mathrm{R}$

Hopo $=H$

ishape $=2$

Edu=Balance ( $i b u$, sofac)

Reri=der

Ras (is) = Rcri

ishape $=3$

$E d u=B a l a n c e$ ( $i b u$, sofac)

Her $i=d$ er

Has (is) =Hcri

c

If(itpr.eq.1) write $(8,1634)$ sofac,Ene,Ropo,Hopo,Rcri,Hcri

1633 continue

c

1634 format $(f 6.3, f 12.1,4 f 12.2)$

c

itest $=$ itpr

if(itest.eq.0) go to 1631

c compute curves Eavailable as function of radius and Height of core and

c $S / F$ in order to illustrate the user about the options.

c

do 5782 is=1, isof 1

$\operatorname{sofac}=\operatorname{sof} 1(\mathrm{is})$

c

c

write $(8,5783)$ sofac, Ereq

5783 format (//' AVAILABLE ENERGY(MWDAYS) AS FUNCTION OF $S / F$ AND R, H'/ $\star / \cdot S / F={ }^{\prime}, f 8.4 /{ }^{\prime}$ Required Energy (MWd)', $f 10.2 / /$

$\star^{\prime} \quad I^{\prime}, 4 x, \cdot R(C M)^{\prime}, 6 x,{ }^{\prime} R / H^{\prime}, 7 x,{ }^{\prime}$ EAVAI', $4 x,{ }^{\prime} H(C M)^{\prime}, 6 x, ' R / H^{\prime}, 7 x,{ }^{\prime} E A$

*VAI'//)

c

RsO $=\operatorname{Ras}($ is) *der
HsO $=\operatorname{Has}($ is) $\star$ der

$\mathrm{HsO}=\mathrm{Has}$
rnt $=$ ndt

ddr $=(\operatorname{Rop}($ is $)-\operatorname{RsO}) / \mathrm{rnt}$

ddh $=($ HOp $($ is $)-H s O) /$ rnt

$n d t=n d t+1$

do 5782 idt=1, nput

$r i d t=i d t-1$

ishape $=2$

$R s=R s 0+r i d t * d d r$

$R=R \mathbf{s}$

Ener $=$ Balance (ibu, sofac)

$x \Sigma a=R / H$

ishape $=3$

$\mathrm{Hs}=\mathrm{HsO}+\mathrm{ridt} \star d d h$

$\mathrm{H}=\mathrm{Hs}$

EneH=Balance (ibu, sofac)

$x h=R / H$

c

write $(8,5784)$ idt, Rg, xra, Ener, Hs, xh, EneH

c

5784 format $(i 5,2(f 9.2, f 9.3, f 12.0))$

c

Page A-G 


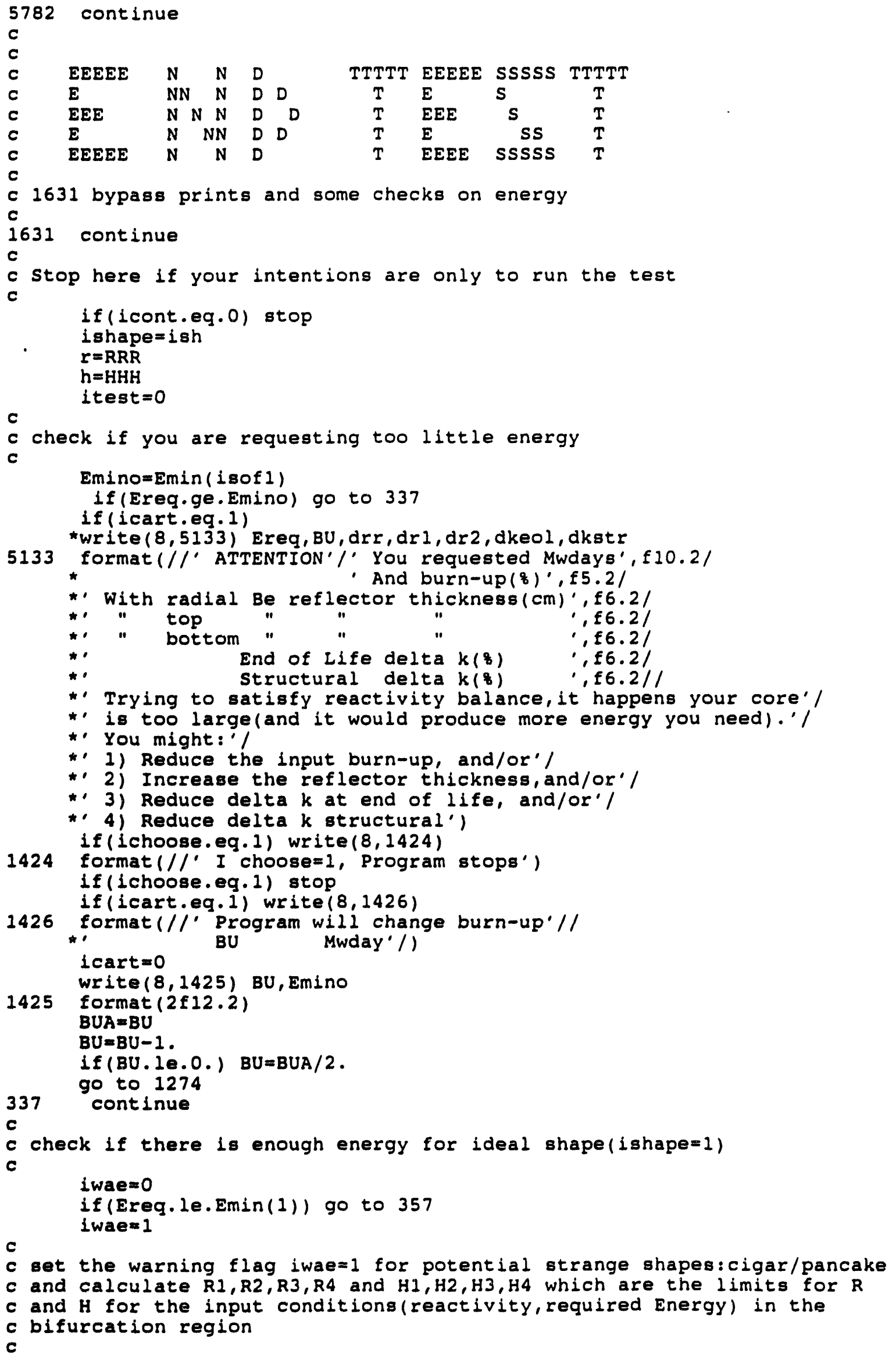




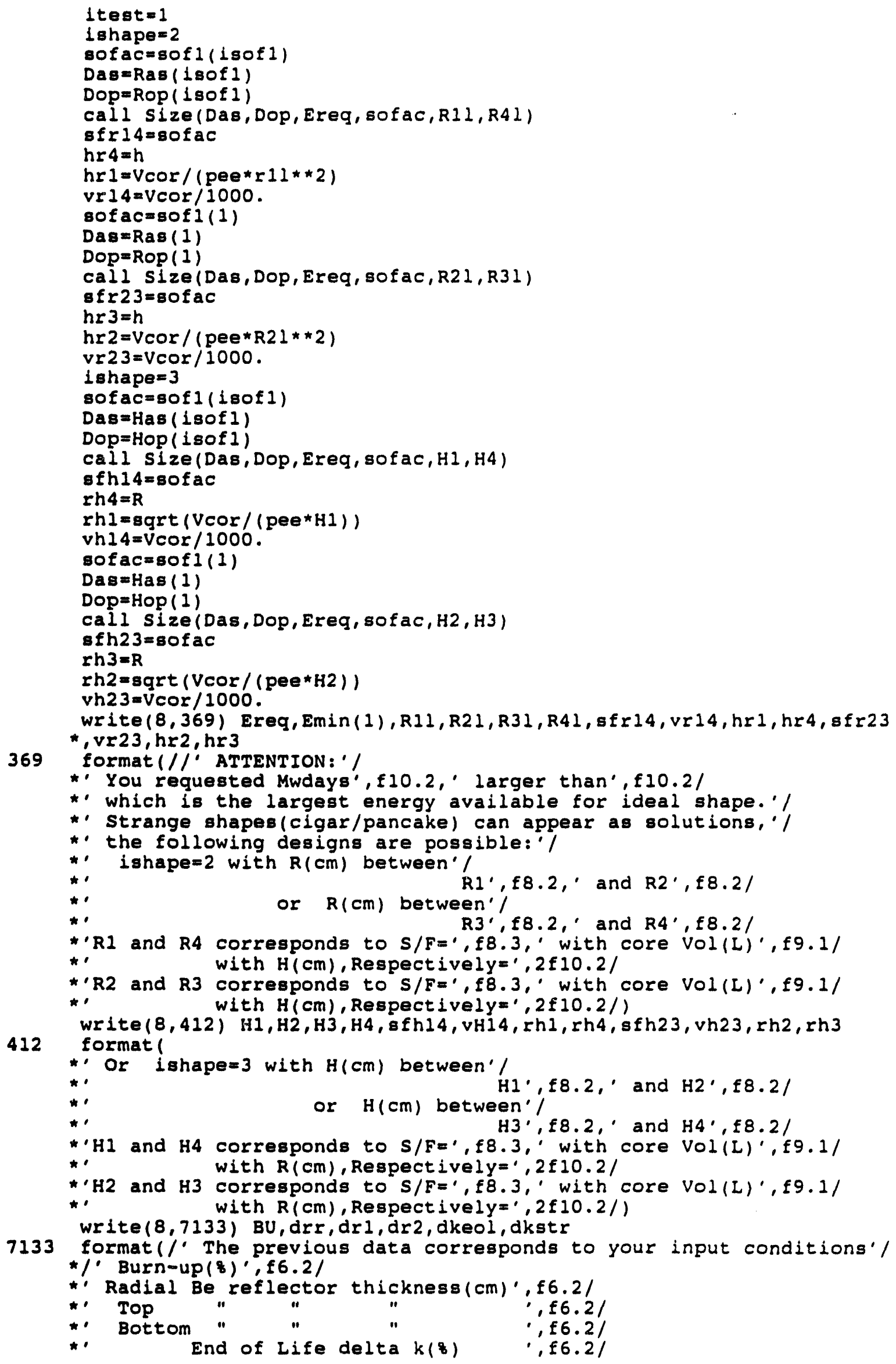




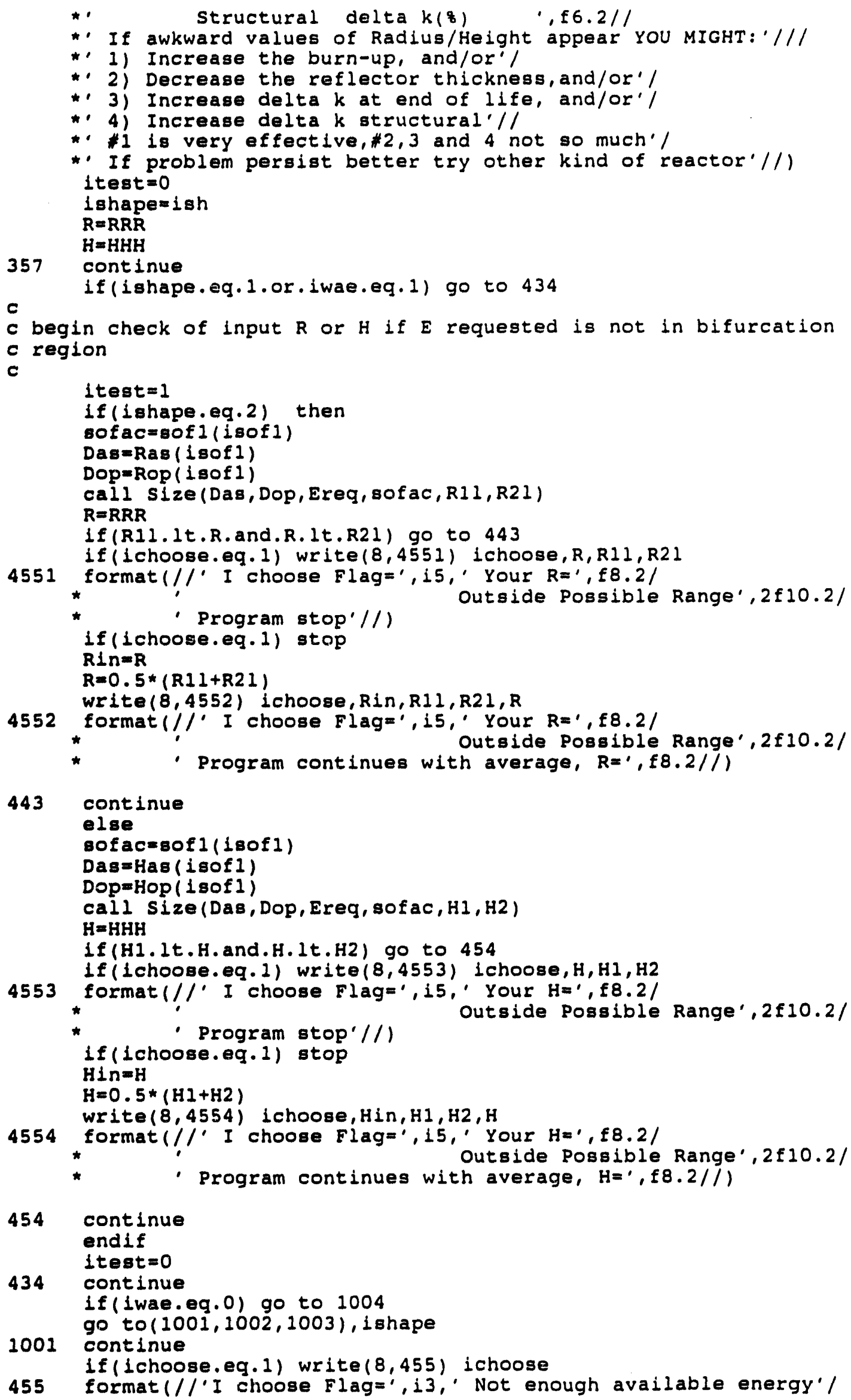




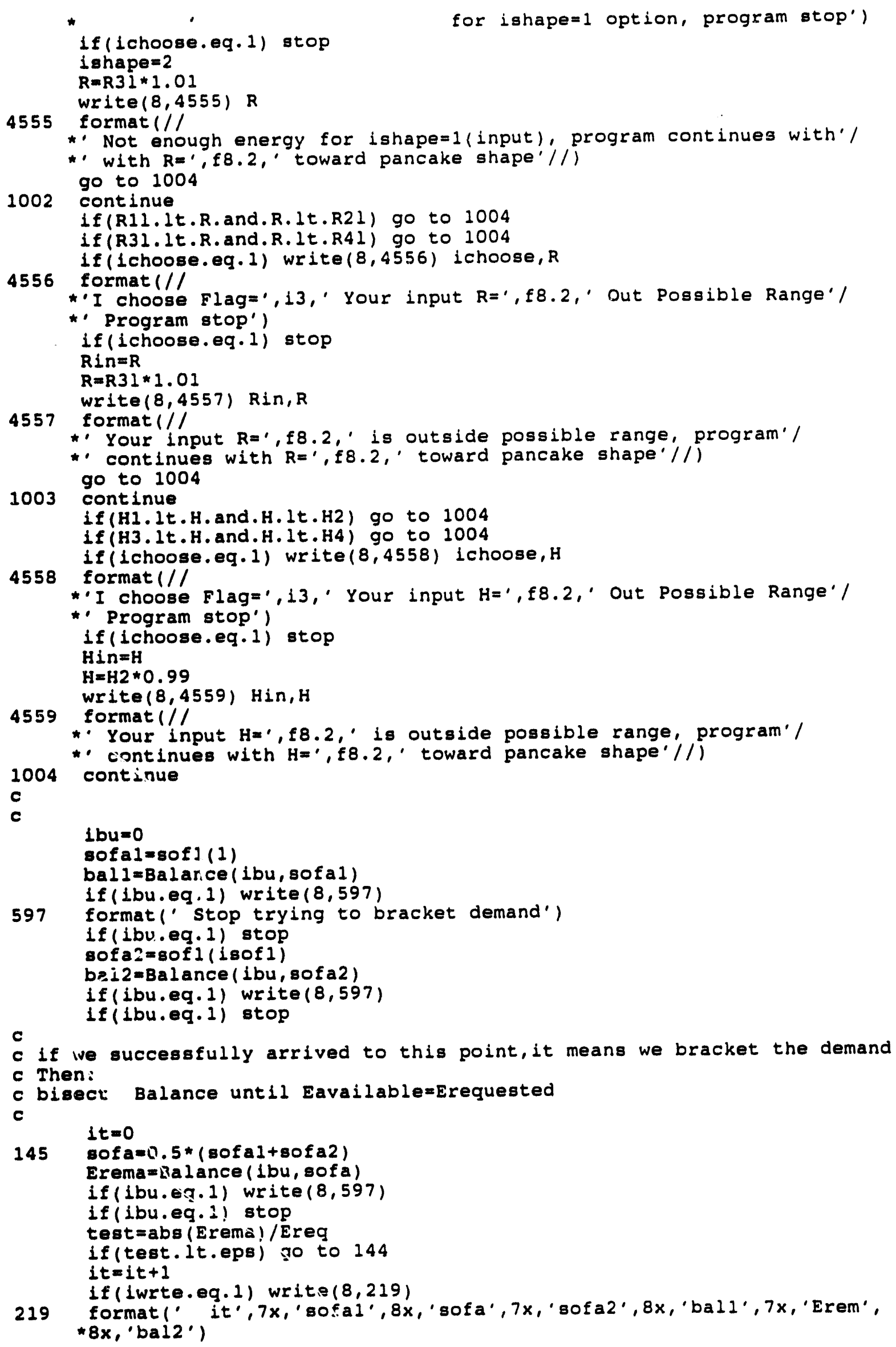


if (iwrte.eq.1) write $(8,218$ ) it, sofal, sofa, sofa2, ball, Erema,bal2

format $(14,3 f 12.6,3 f 12.3)$

call bisect (sofal, sofa2, sofa, bal1, bal2, Erema, iwa)

if (1t.le.itmax. and. iwa.eg.0) go to 145

144

continue

if (it.gt.itmax.or. iwa.eq.1) then

write $(8,146)$ sofal, ball, sofa2, bal2, it, iwa

146

format $(/ / /$ " Problems trying to bisect Balance'/

*

$\star$

$\pitchfork$

- sof $1=$, e12.4, ball=',e12.4/

$\star$,

atop

else

continue

endif

c successful end of reactor search, calculate number densities and masseg

GU2 $35=U 5 m$

Vact $=0.001 * \operatorname{Vcor} /(1 .+80 f a)$

$U 5 \mathrm{~m}=\mathrm{U} \mathrm{m} / 1000$.

rasp=sofa

sof $r=80 f a$

VCaveor

c

c with power level, $8 / f$ and volume of the core, compute fluxes and

c reactivities of $\mathrm{Xe}$ and $\mathrm{Sm}$

Call Elux

C choose $R$ as near possible to ideal $R$ with integer number of elements $a e=0.86602540 * f \in * * 2$

ne $=$ pee* $R * 2 /$ ae

$r e=n e$

if (rasp.eq.0.) then

$n f=n e$

$r f=n f$

nse $=0$

ree $=0$.

go to 1975

else

$r f=r e /(1 .+r a s p)$

$n f=r f$

$n s e=n e-n f$

rbe=nse

endif

1975 Resgrt (ne*ae/pee)

$H=V \operatorname{cor} /($ ne*ae)

Hact $=\mathrm{H}$

c

c fuel microcell, calculation of geometry and concentrations

c aseume clad around coolant hole and around fuel element

c aae is the active area of ae, acl is the area of the clad around hole

$c$ and ac2 is the area of the clad around fuel element

c aco is the area of the coolant hole

c

$d c c=0.1 * d c$

$r h=d h * 0.1 / 2$

$\operatorname{ac} 1=p e{ }^{*} d c c *(2 . * r h+d c c) \star n h$

$b=f f / 1.7320508$

$b p=b-d c c / 0.86602540$

$a c 2=3 . *(b+b p) * d c c$

acompee*rh**2*nh

aaemae-ac1-ac2-aco

c volumetric fractions

c

Page A-11 


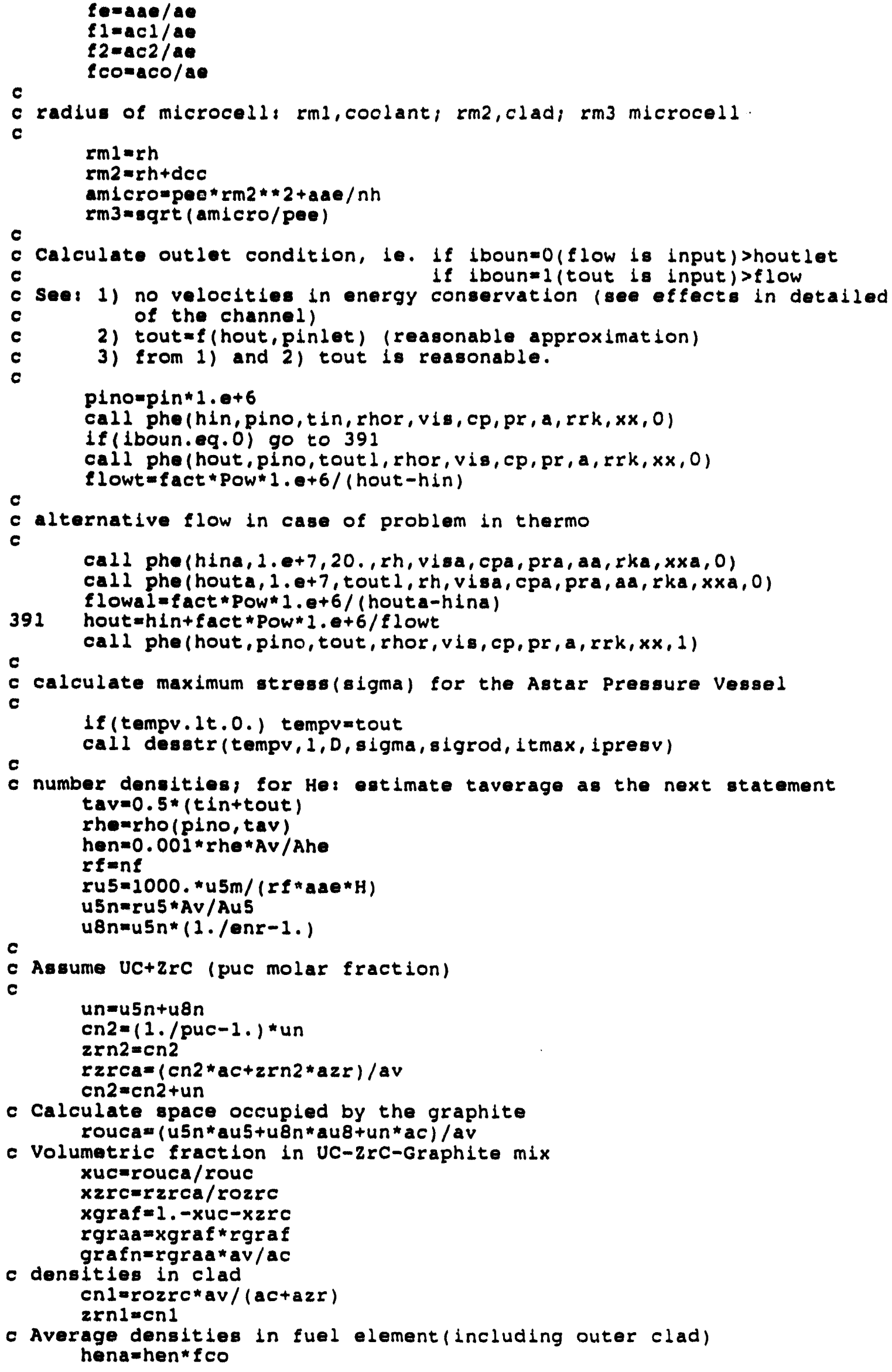




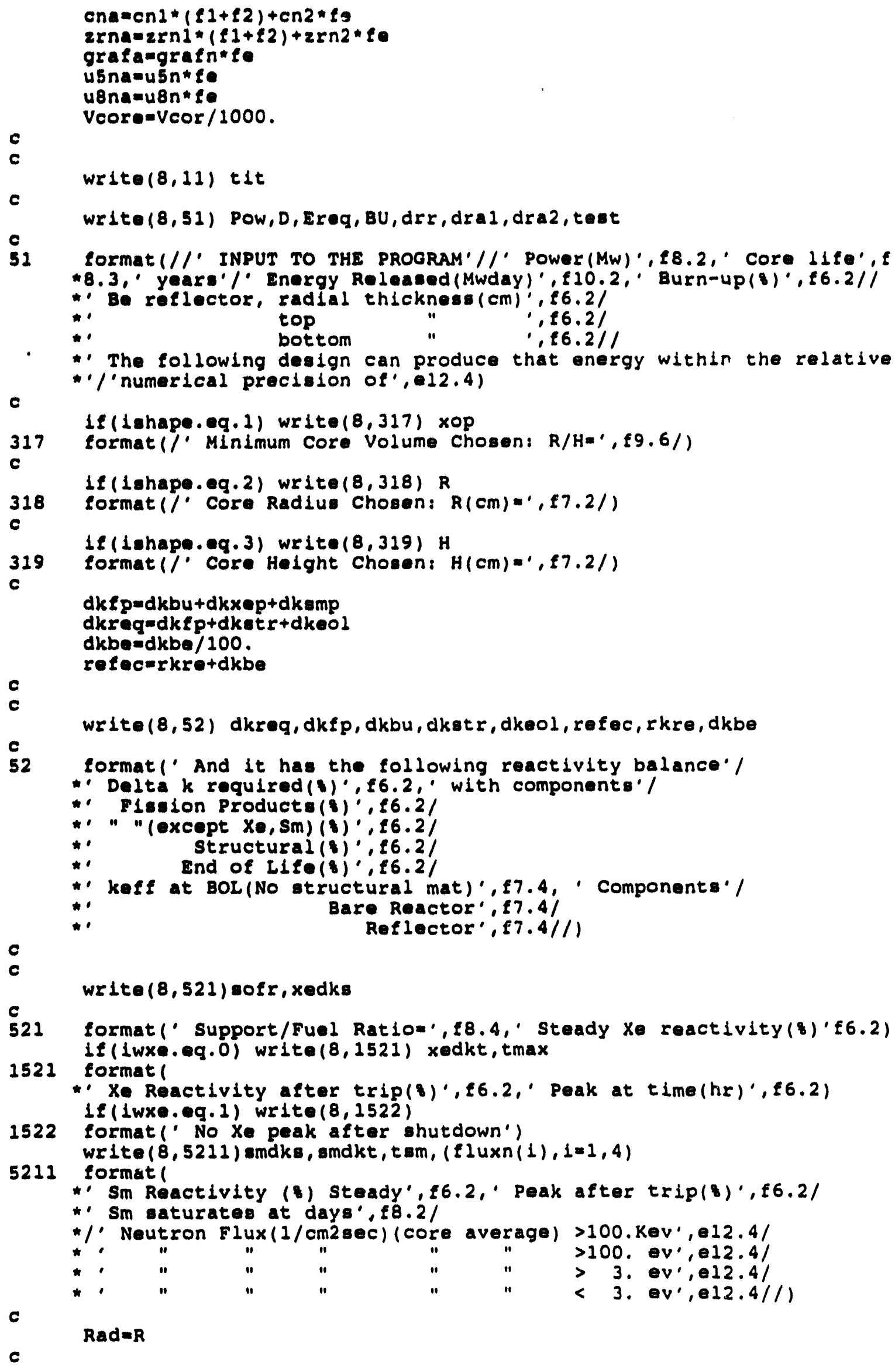


c call control to design control drums

c

call control

c

c

wrlte $(8,30)$ usm, enr, Vcore, rasp, $R, H$, ne, nee, nf, ff

c

write $(8,40)$ puc, dc, $n h, d h, p i n, t a v$

40 tormat! FUEL ELEMENTS(INDIVIDUAL) $\%$

" Molar uc eraction in UC-zrC mix $10^{\prime}, f 7.4,{ }^{\prime}$ zrc clad thickness

*(mm) $18^{\circ}, 16.21^{\circ}$ There are $, 14,^{\circ}$ coolant holes of $\mathrm{mm}^{\prime}, \mathrm{f} 7.2,^{\circ}$ diamet

*er' $/$ with He coolant at', $f 7.3,^{\prime} \mathrm{MPa}^{\prime}, f 8.2,{ }^{\prime} \mathrm{K} / \mathrm{l}$

c

c

wrlte $(8,50) f c 0, f 1, f 2, f e$

$c$
50

format(' Volume fractions in Fuel Element'/' Coolant', $f 7.4 /$

* ${ }^{\prime}$. Clad Coolant, 17.4

$*$
$*$

c

c

c

write $(8,41)$ xuc, rouca, xzrc, rzrca, xgraf, rgraa

41

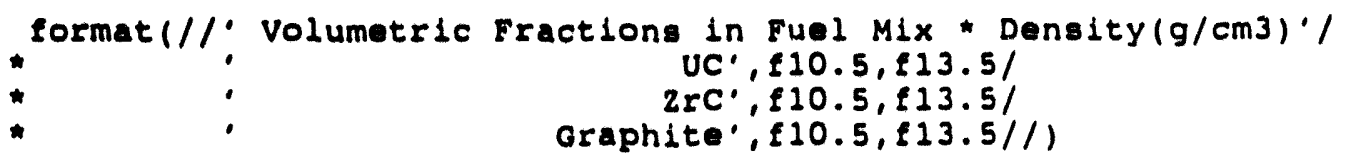

c

c

write $(8,60) \mathrm{rm} 1$, hen, $\mathrm{rm} 2, \mathrm{cn} 1,2 \mathrm{rn} 1, \mathrm{rm} 3, \mathrm{cn} 2,2 \mathrm{rn} 2, \operatorname{grafn}, \mathrm{u} 5 \mathrm{n}, \mathrm{u} 8 \mathrm{n}$

60

60

format (//. Fuel microcell (Number Densities $10 * 24$ atoms/cm3) '/

- Excludes outer cladding'/

- Coolant Radius(cm)', $f 7.4,^{\prime} \mathrm{He}^{\prime}, \mathrm{fl2.8/}$

- Clad Radius (cm)',f7.4,' C',f12.8,' , $^{\prime}, f 12.8 /$

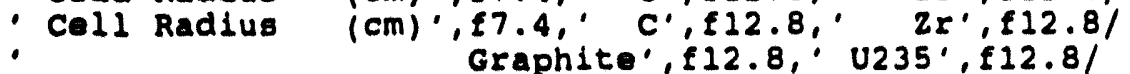

Graphite , f12.8, U235', f12.8/

c

c Total masa of the fuel elements

Vact $=r f * a e * h / 1000$.

ofuel =Vact * (hena "ahe+cna*actzrna*azr+grafa*ac+u5na*au5+u8na*au8) /a

*v

Ggraf =Vact*grafa*ac/av

ozrCc=Vact * $(f 2+f 2)$ * rozrc

GHe=Vact * hena*ahe/av

GZrCf =Vact *fe* rzrca

GUC=Vact * fe* rouca

WUC=GUC*100. / G fuel

w2rCf mazrCf +100 . /Gfuel

woraf =Ggraf*100./Gfuel

w2rCc=GZrCc*100./G /

WHe $=$ GHe* 100 . / G fuel

rou $5=u 5 m * 1000$. / Vact

c

Page A-14 


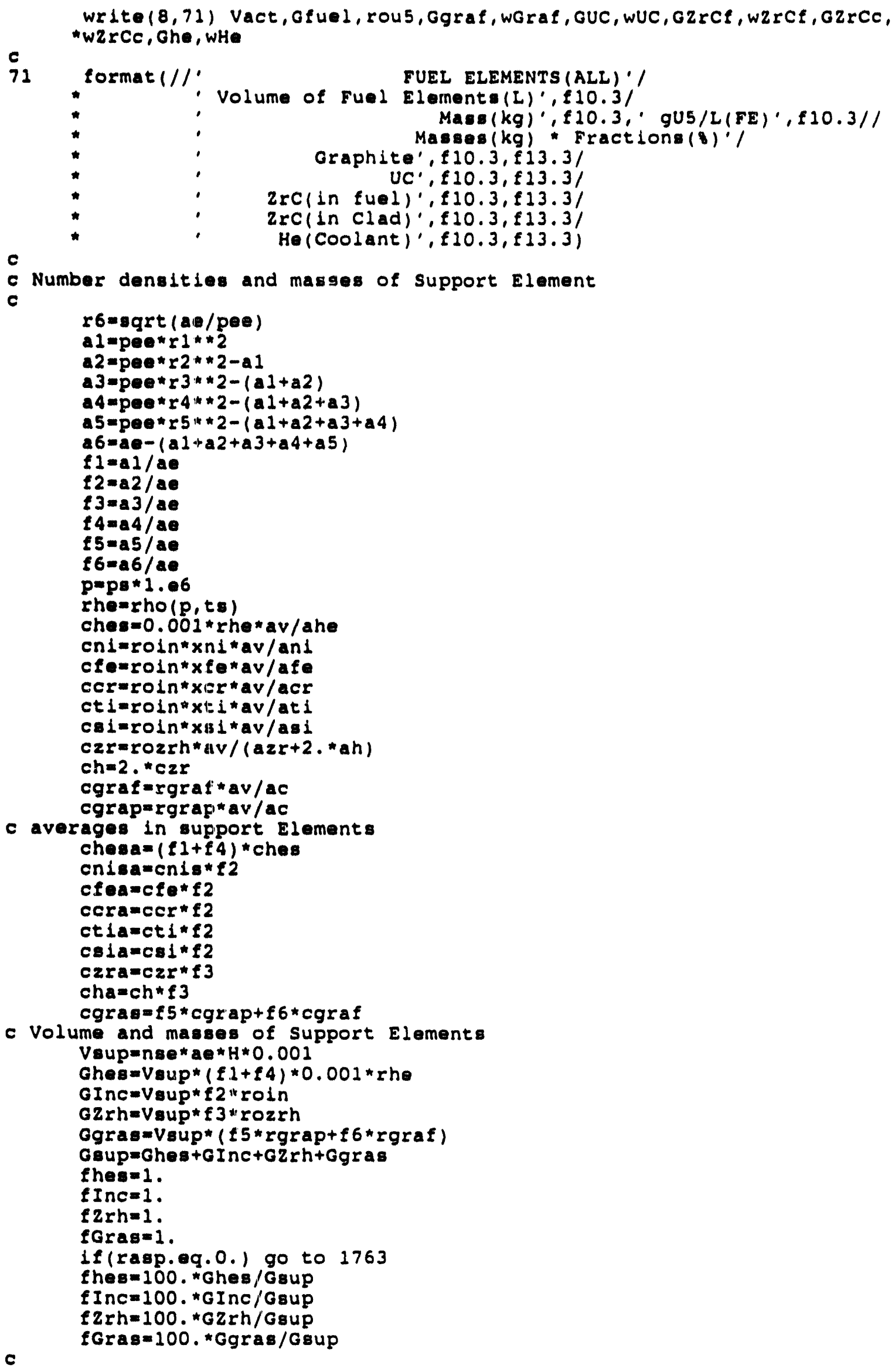




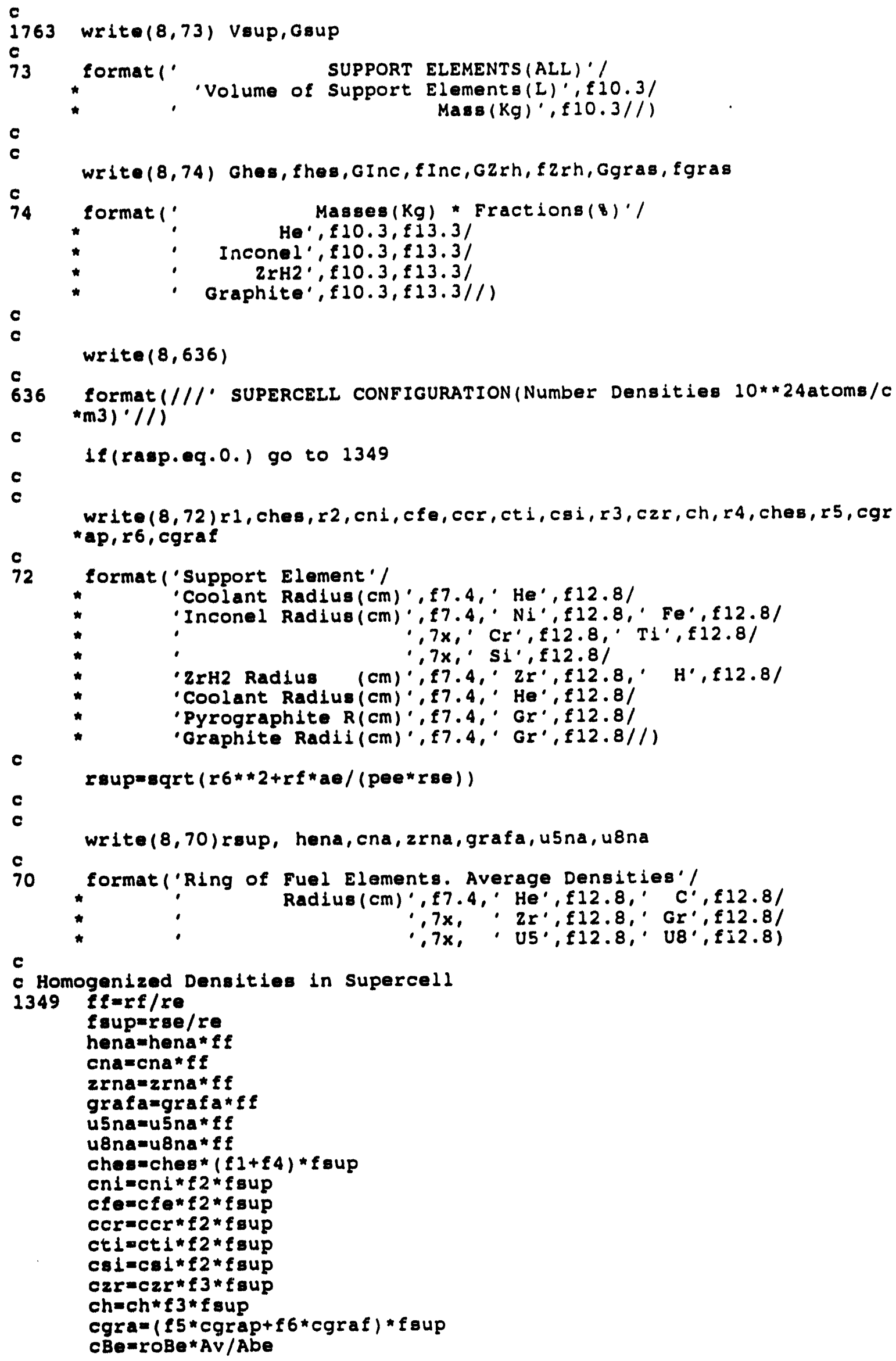




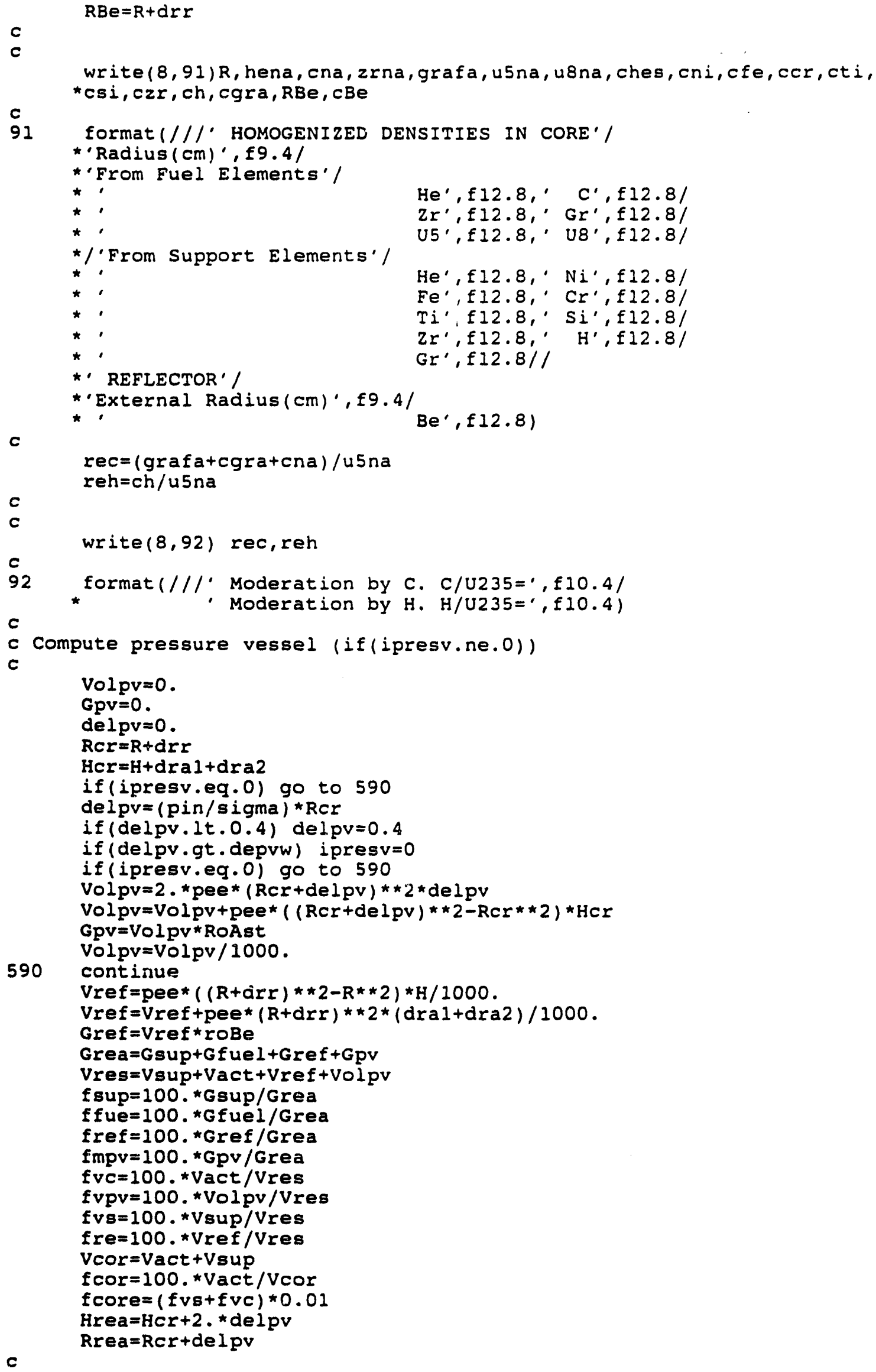




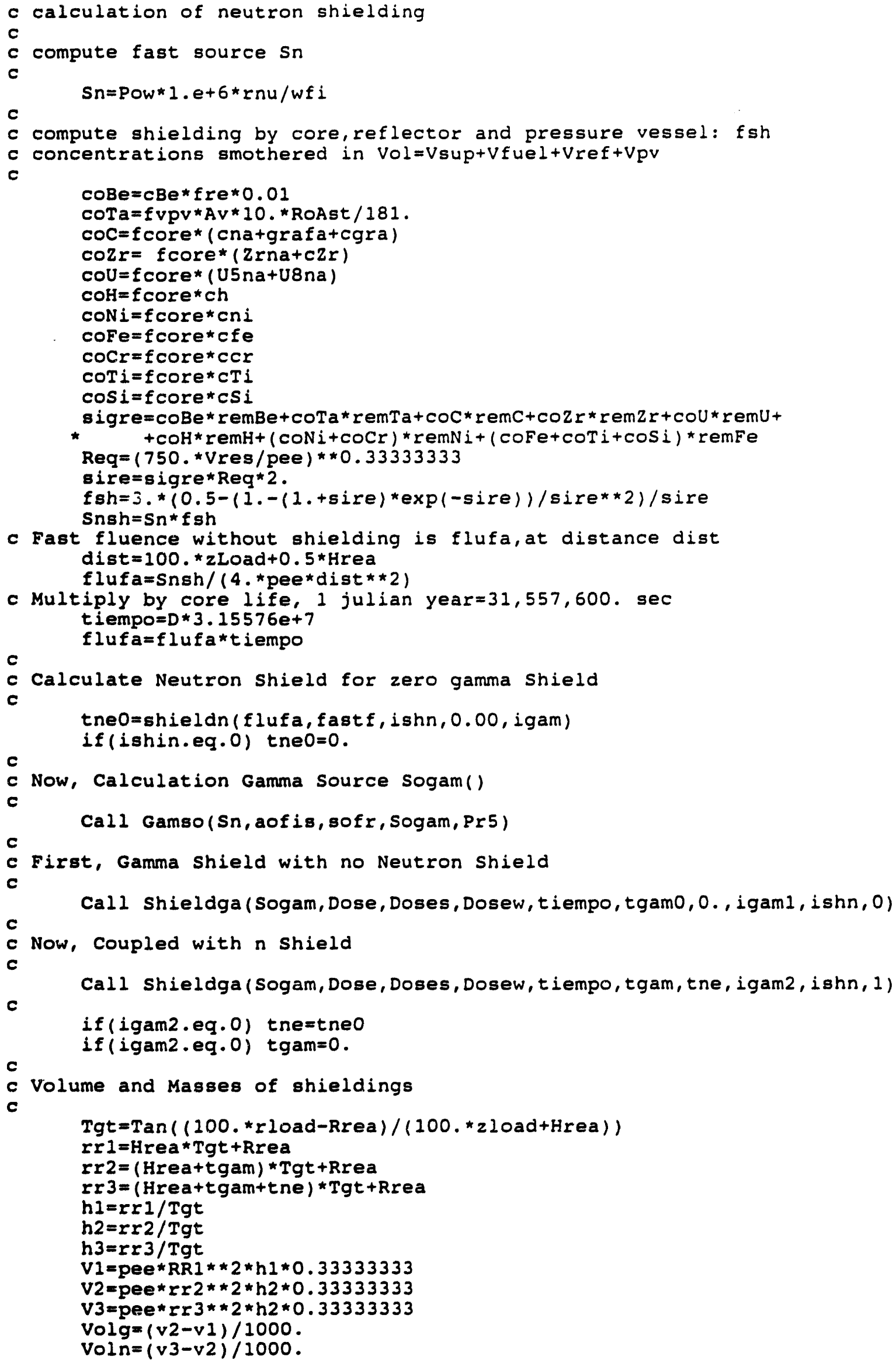

Page A-18 


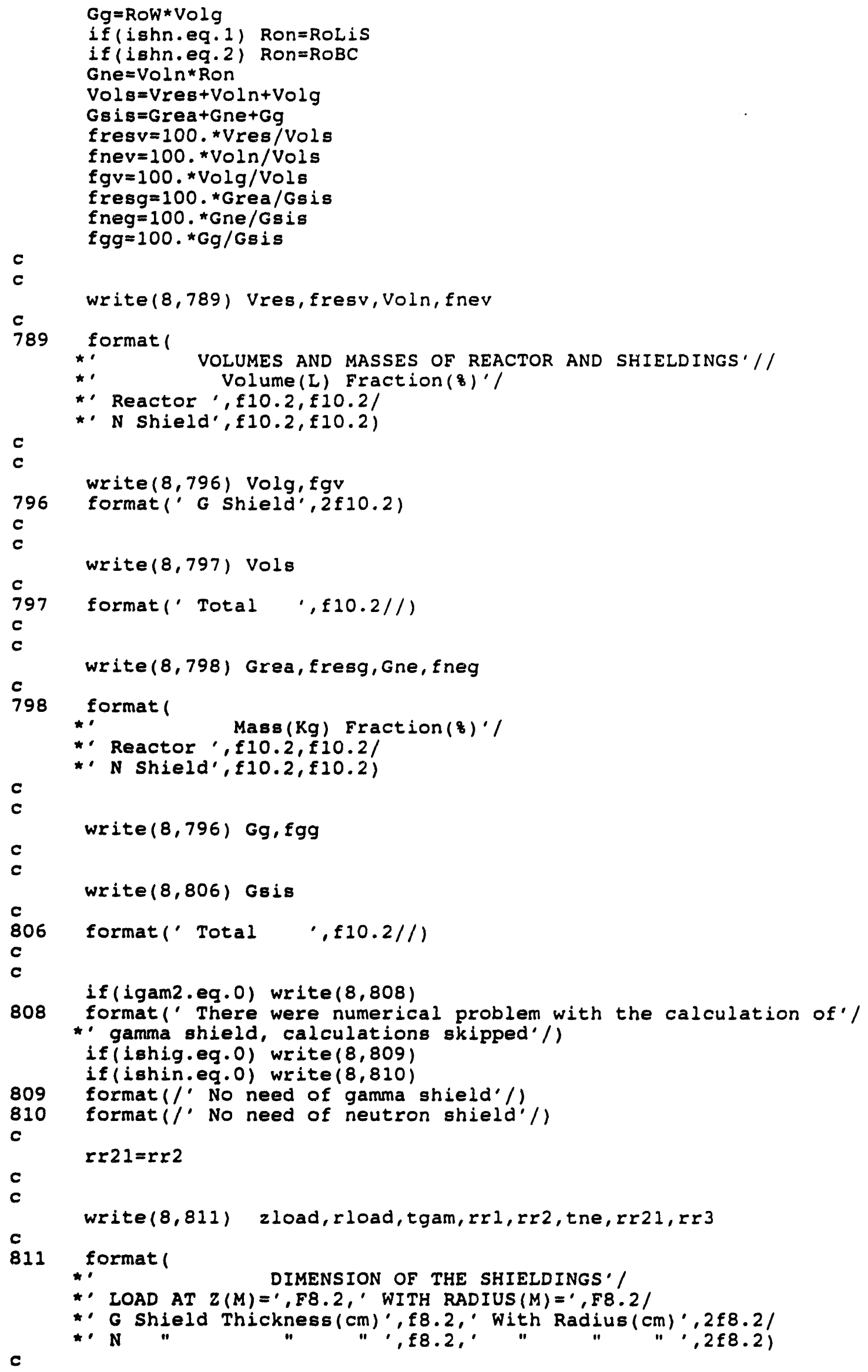



775

c

c

776 format(' Integrated dose with shieldings(W and Neutrons), Total (R *ads)',e12.2/

* Tungsten Thickness(cm)', f10.2/

* Tungaten Thickness $(\mathrm{cm})^{\prime}, \mathrm{f} 10.2$, ' if no Neutron Shield Present'//)

if (ishig.eq.1. and.igam2.ne.0) Dosos=Dogeg

if (ishig.eq.0) Dosos=Dosen

write $(8,776)$ Dosos, tgam,tgamo

c

c

804 format (/' Requested Dose(Rad)', f10.2,' at ',f6.2,' meters'/)

C SOLVE THERMALHYDRAULIC 


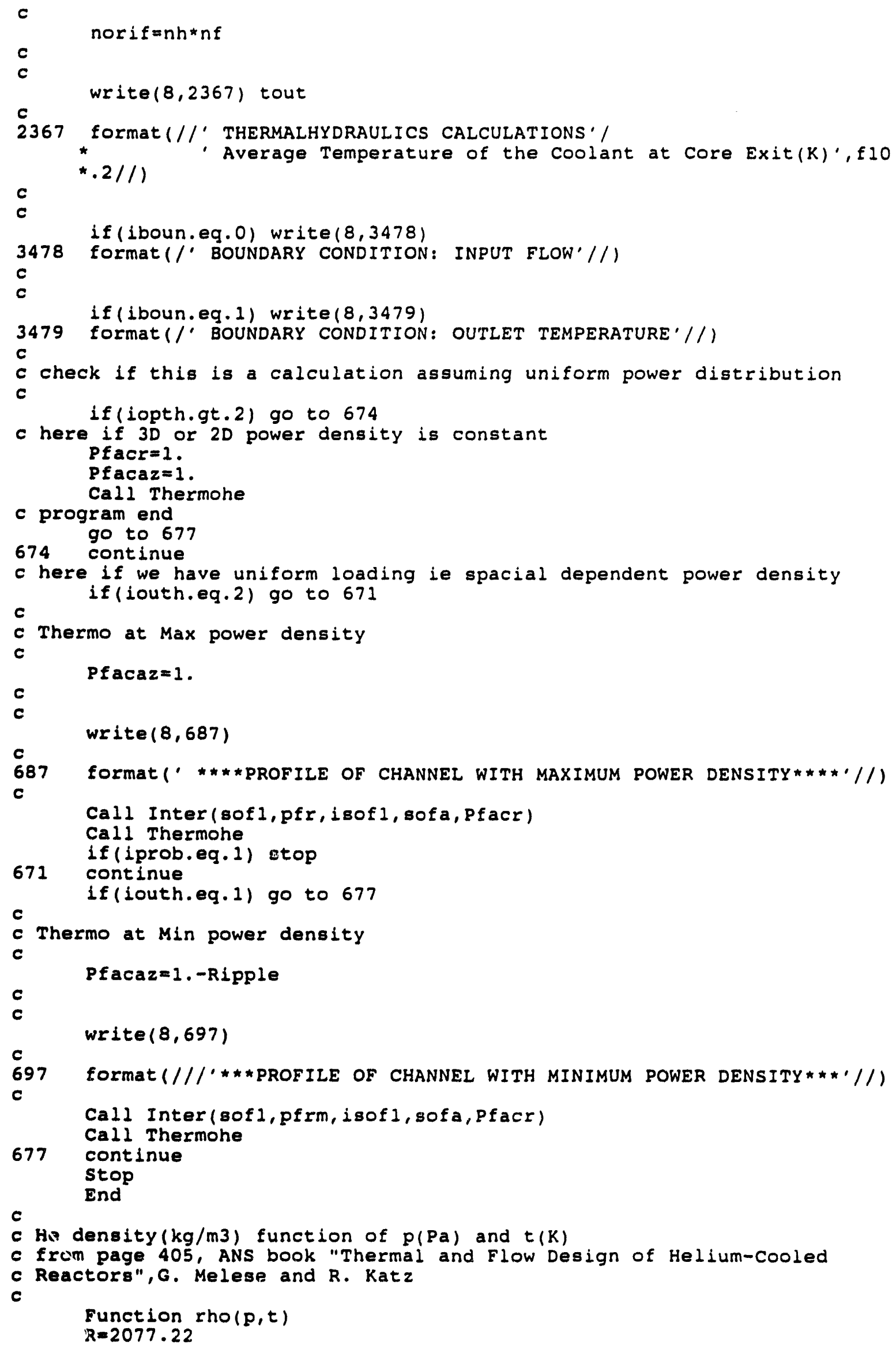




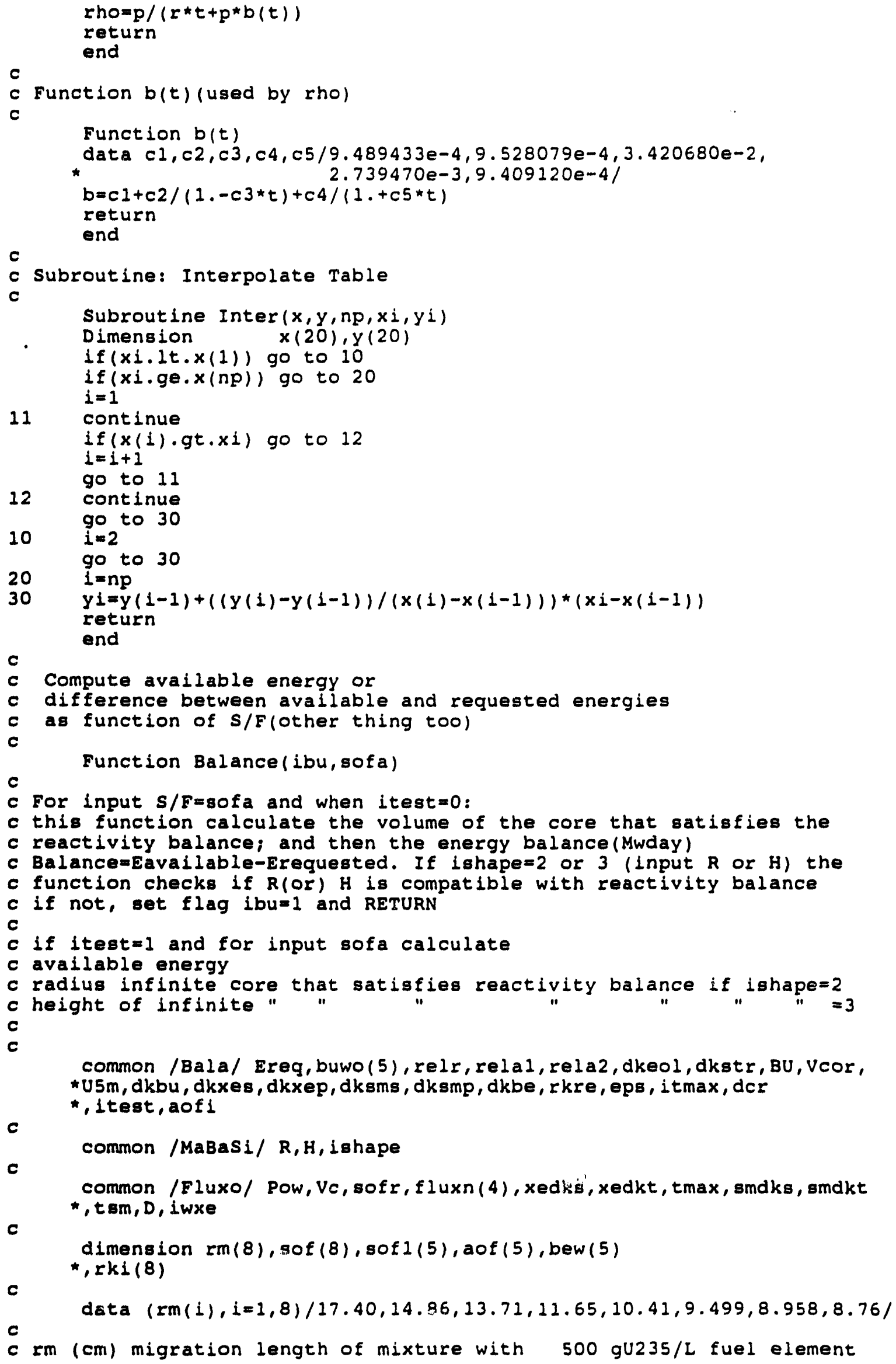




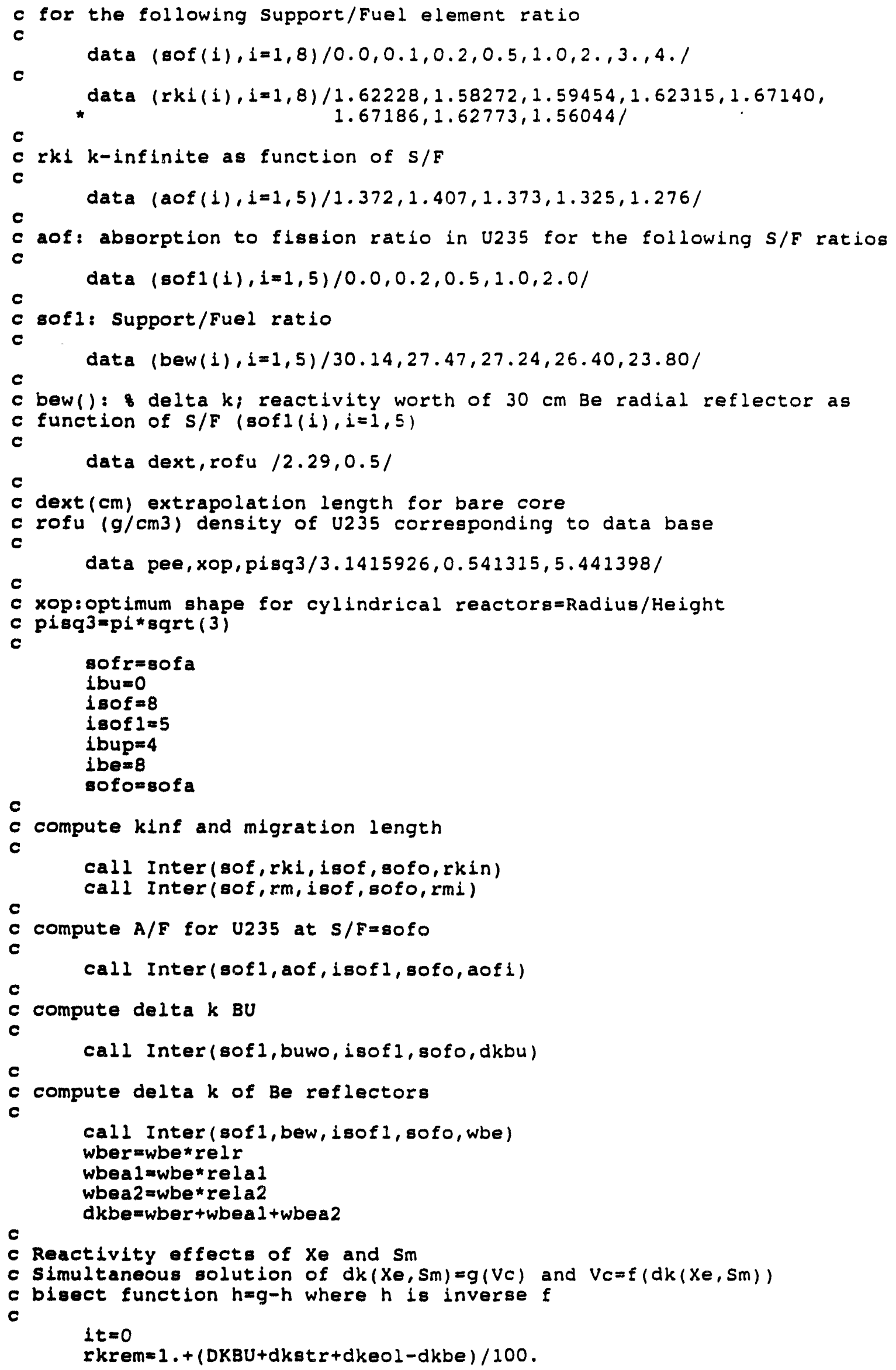




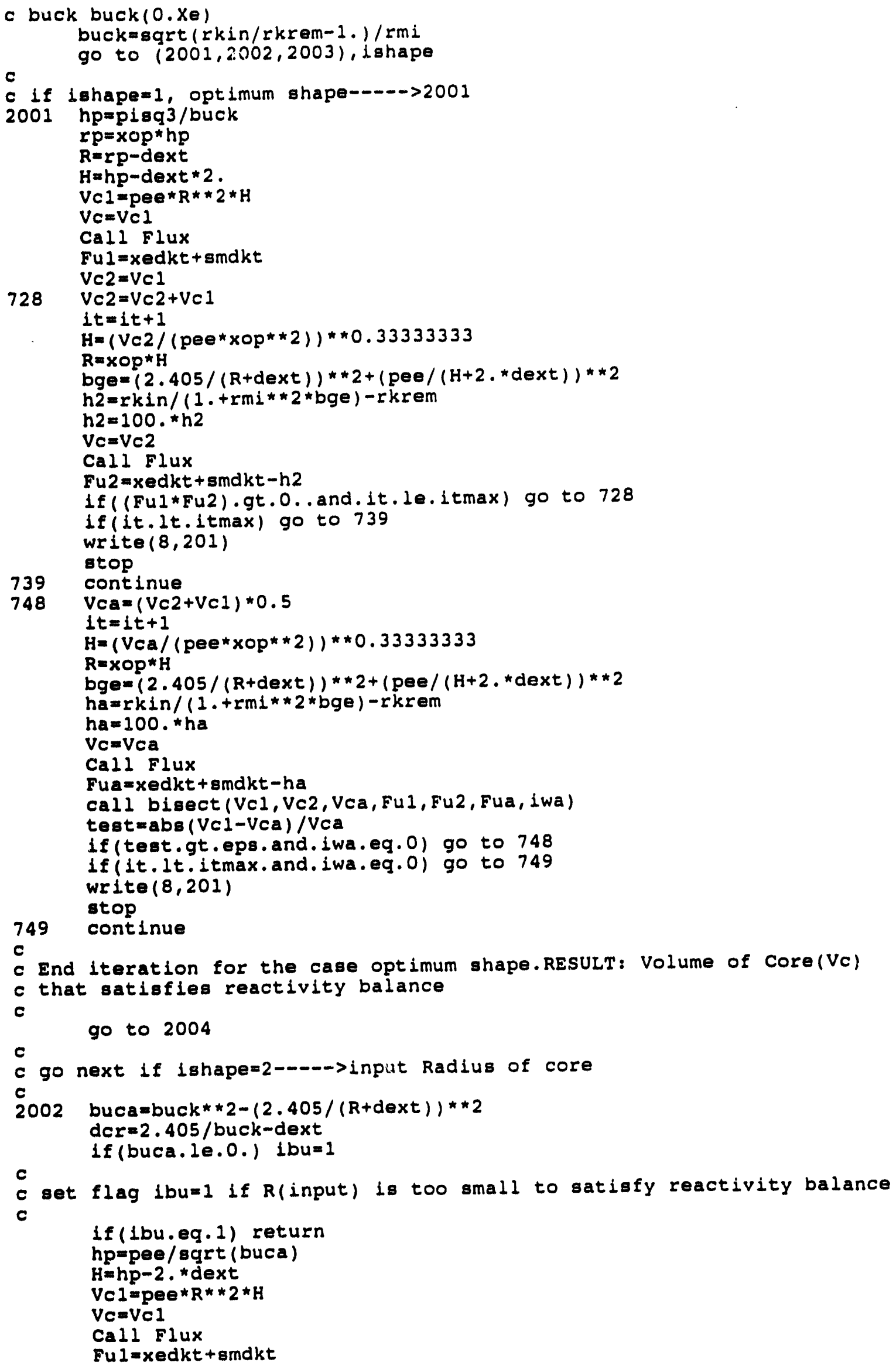




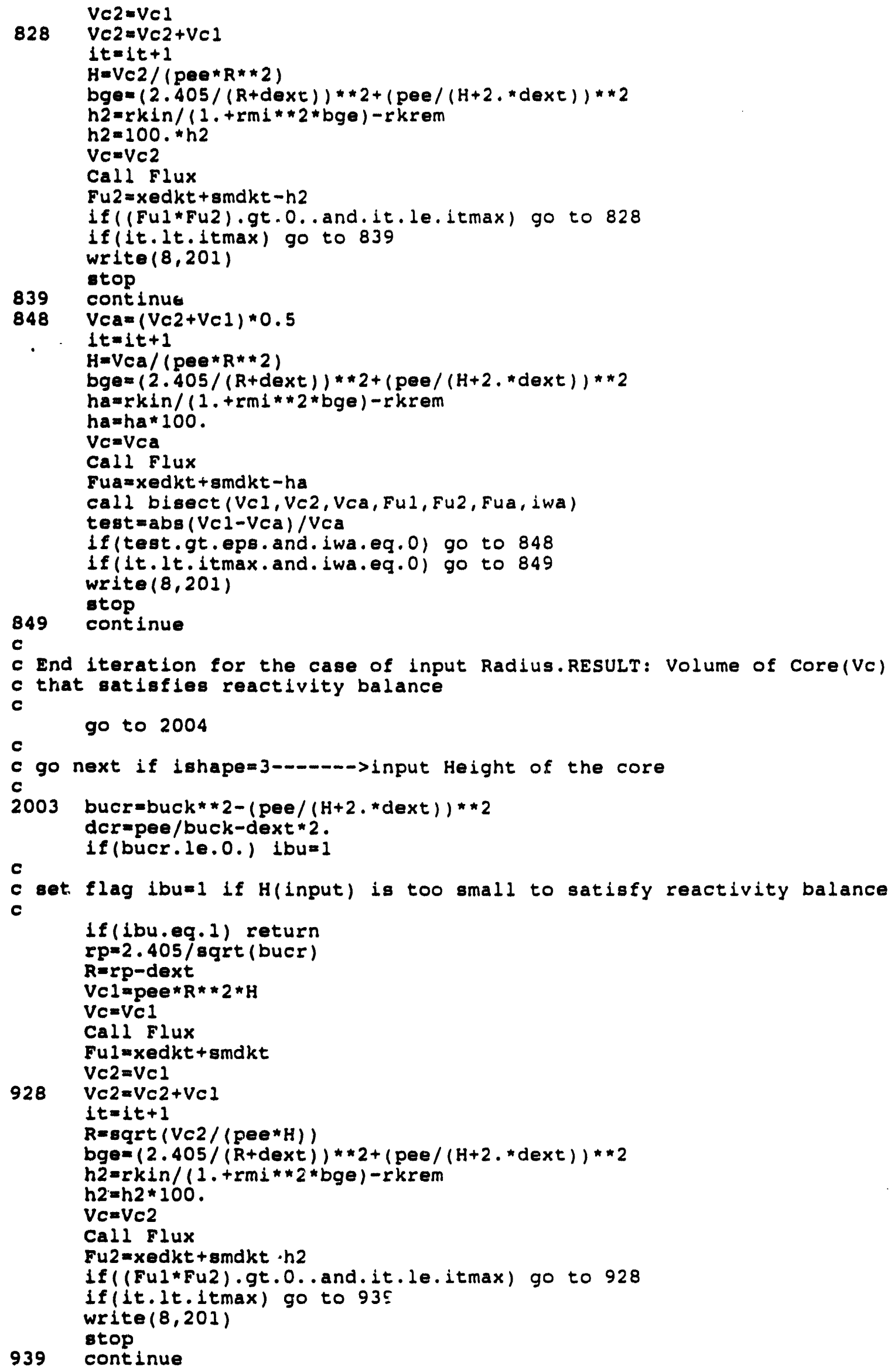




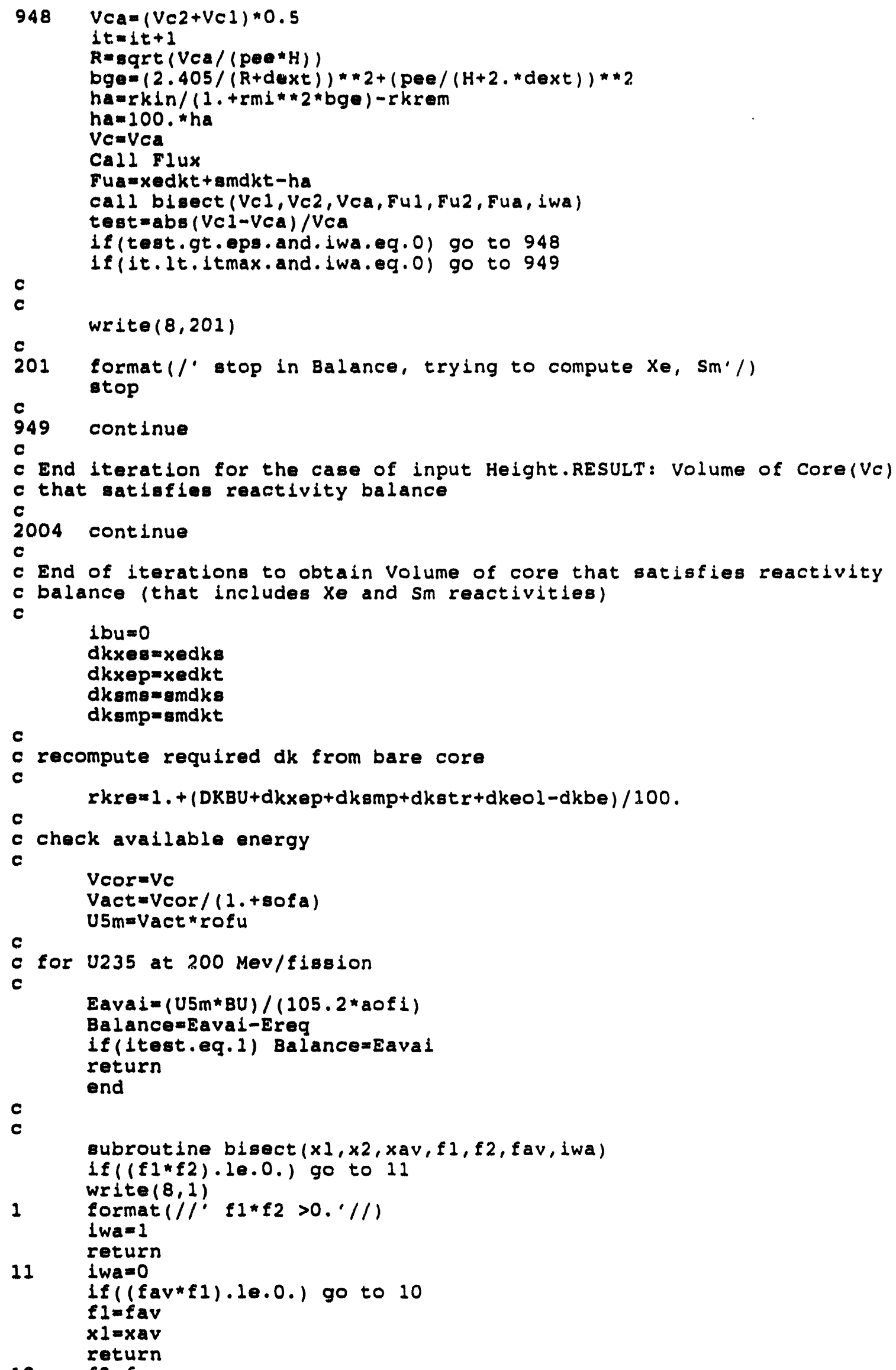




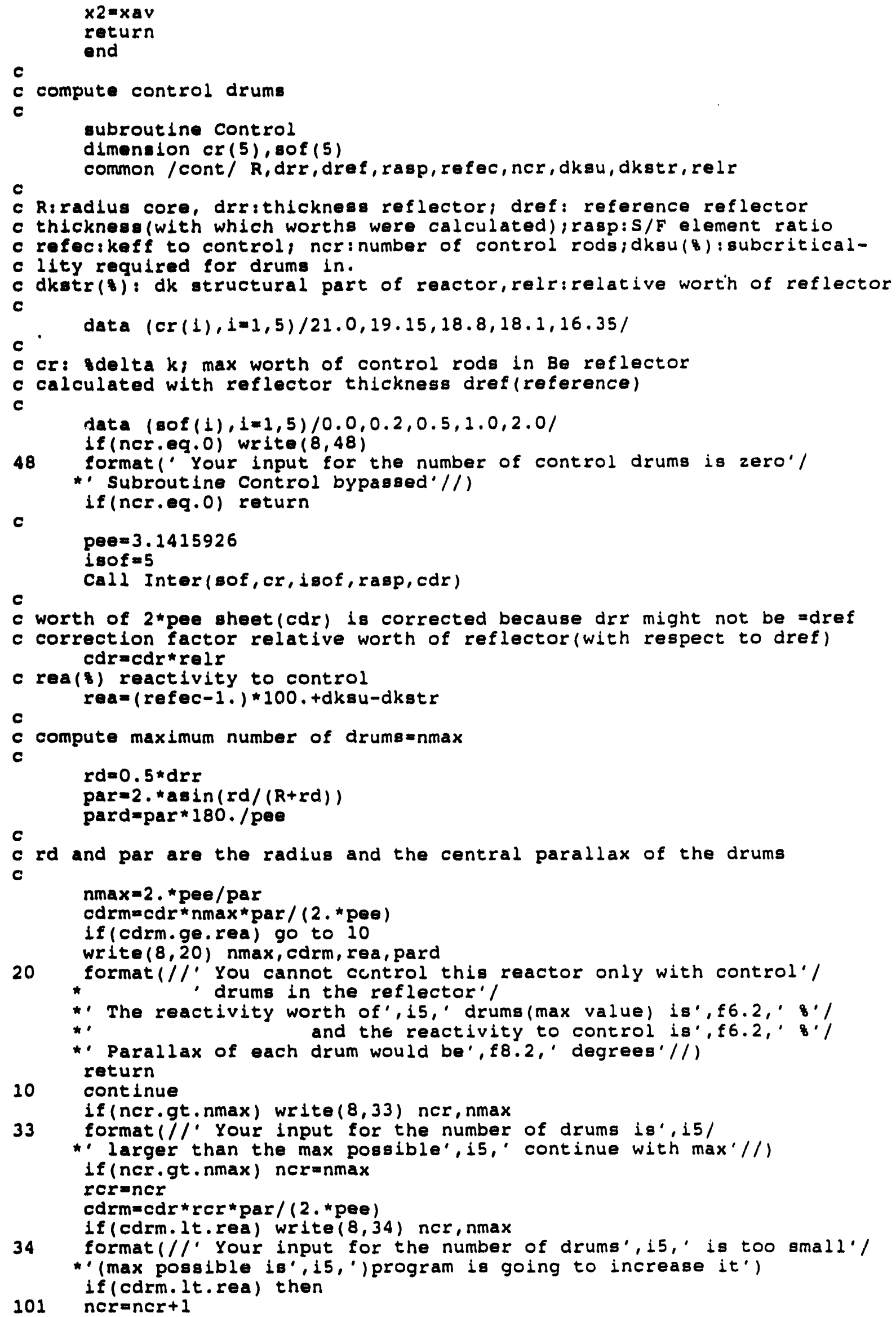




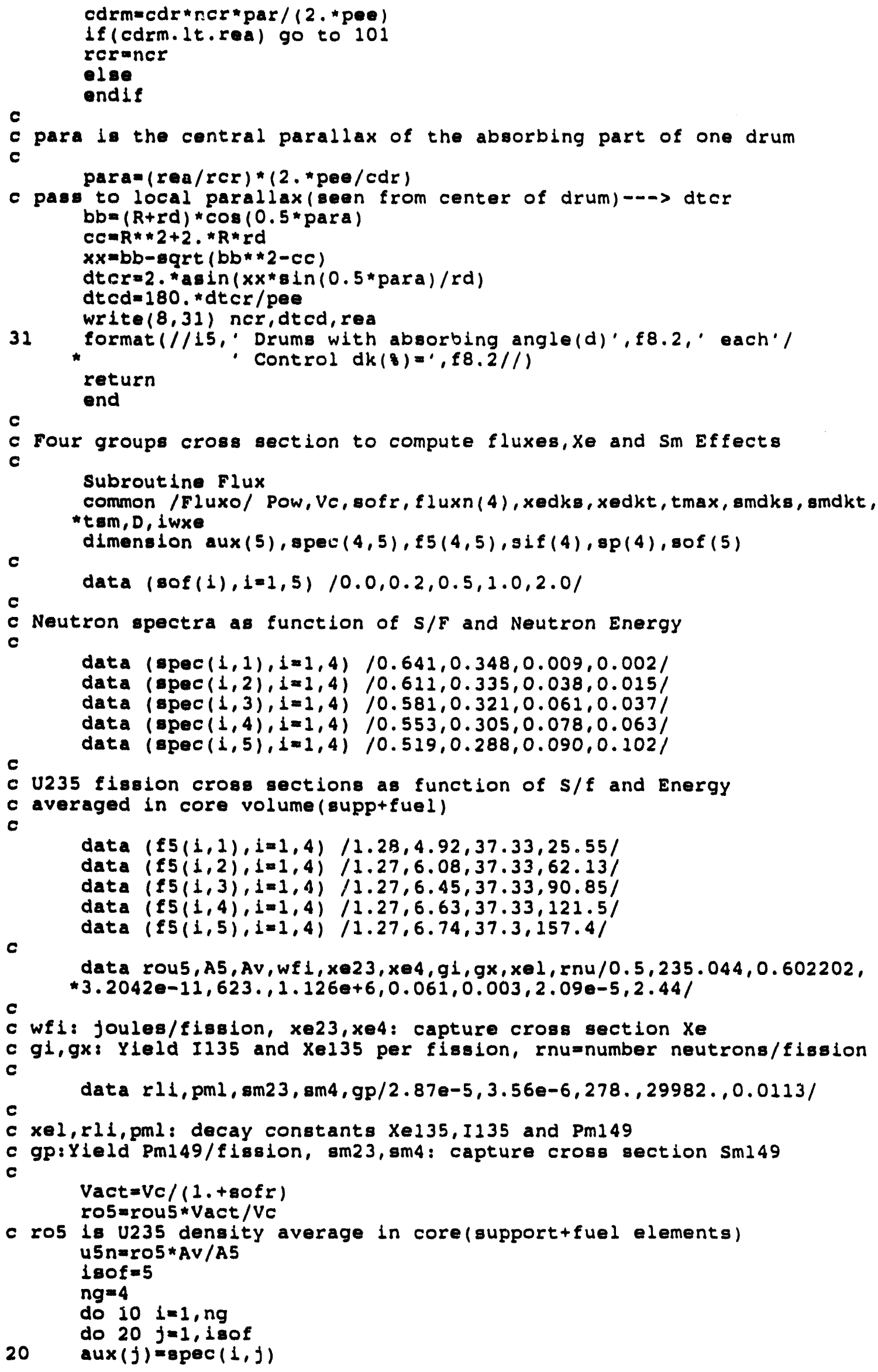


call Inter (sof, aux, isof, sofr, spe)

$\operatorname{sp}(1)=\operatorname{spe}$

do $30 \mathrm{j}=1$, isot

30

$\operatorname{aux}(j)=f 5(i, j)$

call Inter (sof, aux, isof, sofr, sig)

10

eif $(1)=8 i g$

continue

$\lg f=0$.

do $40 \quad 1=1, \mathrm{ng}$

40

elgf=glgftgp(1)*g1f(1)

Bigf=glgf*usn

flun=Pow* $1 . e+6 /(V c * w f i * g l g f)$

c flun average flux in core

do $50 \quad 1=1, \mathrm{ng}$

$50 \quad$ Iuxn $(1)=f \operatorname{lun} * \operatorname{sp}(1)$

$x \in c=x e 23 *(\operatorname{sp}(2)+\operatorname{sp}(3))+x \in 4 * \operatorname{sp}(4)$

fluxe $=x e 1 * 1 . e+24 /$ xec

xedks $=100$.* $(g l+g x) * f l u n /($ rnu* (fluntfluxe) )

compute max xe after trip(iwxe=0)

c If no max appears set flag iwxe=1, and make xedkt=xedks

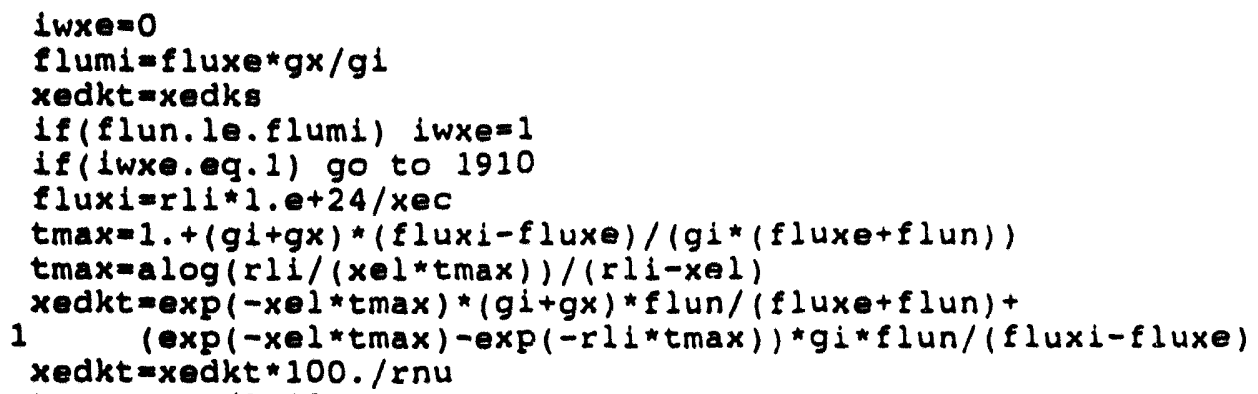

1910 continue

smc $=\operatorname{sm} 23 *(\operatorname{sp}(2)+8 p(3))+8 m 4 * \operatorname{sp}(4)$

smc $=8 m c * 1 . e-24$

flusm $=\mathrm{pml} / \mathrm{amc}$

tam $=1 . /$ (amctflun)

$t v i d a=D * 3.15576 e+7$

c

compute sm after trip

c

$t s m o=t v i d a / t s m$

et $s m=0$

If (tamo.1t.50.) etsm=exp (-tsmo)

smdks $=g p^{*}(1 .-e t s m) / r n u$

smakt $=s m d k s+g p * f l u n /(r n u * f l u s m)$

smdks $=100$. * gmaks

smdkt $=100$. * smdkt

$t 8 m=t 8 m / 86400$.

return

end

solve thermalhydraulic

c

$$
\begin{aligned}
& \text { subroutine thermohe } \\
& \text { real } 4 \mathrm{k}
\end{aligned}
$$

c

dimanaion pow (51), prea (́21), tbulk(51), ve(51), $\operatorname{rmach}(51), 2(51), \mathrm{rj}(51)$

*,tw(51), Tclad(51), Tfuel (51), Tclaa(51), Tfua(51)

c

common/thermo/ aln, nnodo, lexpl, ifri, itmax, norif, iditus, nfu, pin, tin

*,d, ru, xi, der, eps, Powt, rjs, flowt, GU235, Vact, Enr, PUC, Rouc, Rozrc,

* Rograf, delad, Hact, Pfacr, Daxi, fact, phav, thav, fe, iprth, iopth, iouth

* Pfacaz, itry, flowal, iboun 

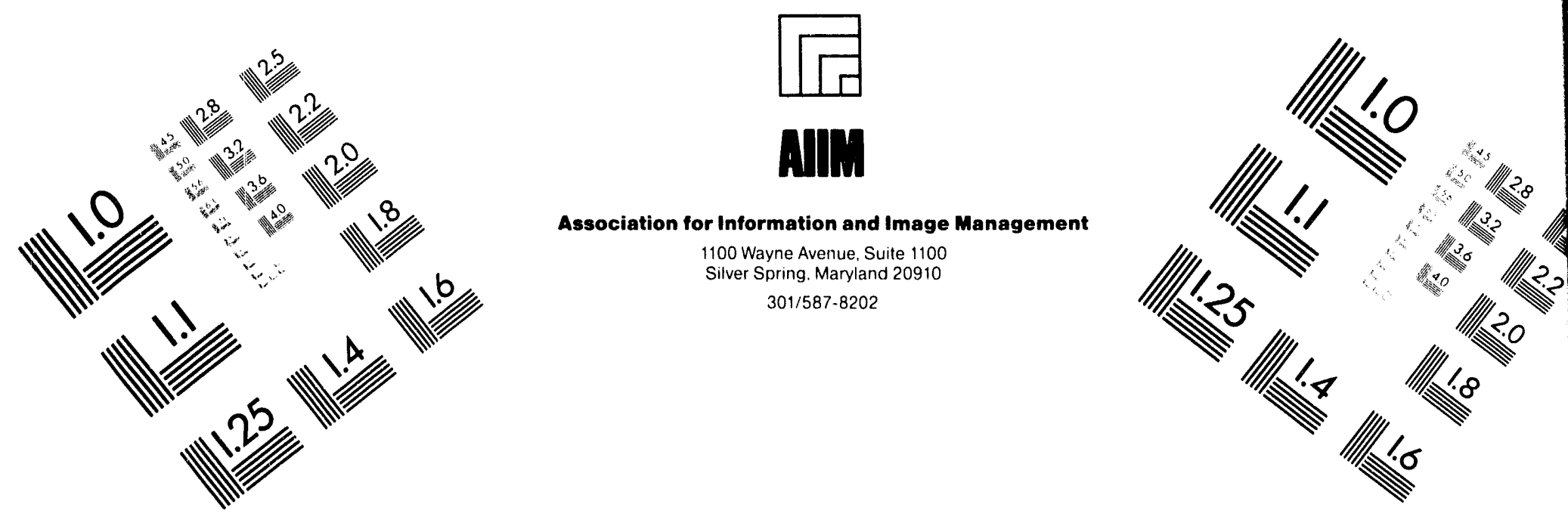

\section{Centimeter}

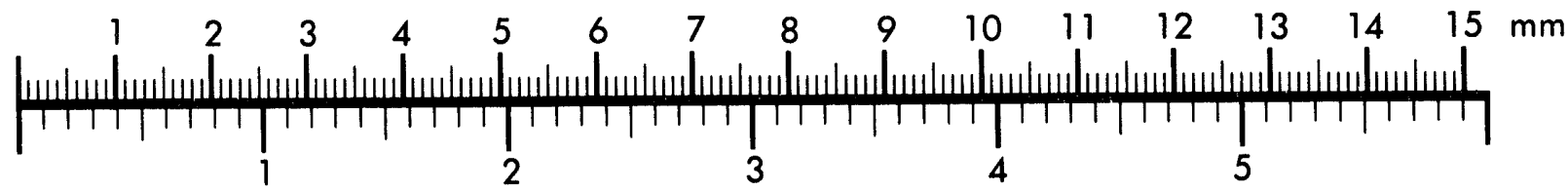
Inches

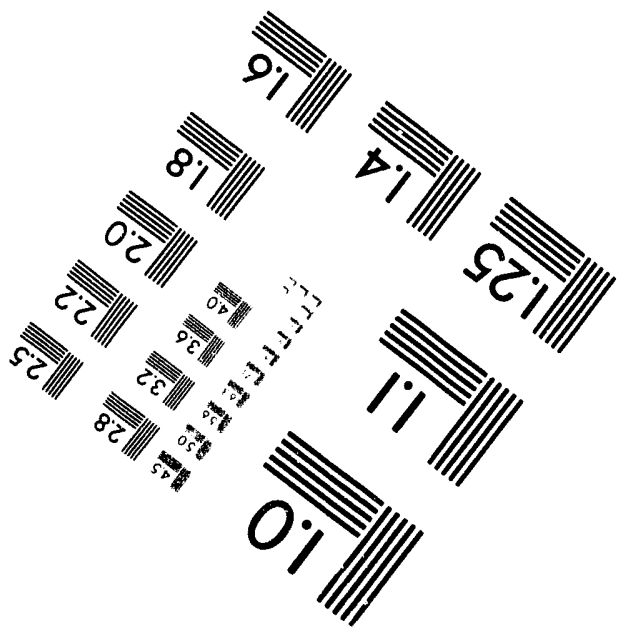

MANUFACTURED TO AIIM STANDARDS

BY APPLIED IMAGE, INC.

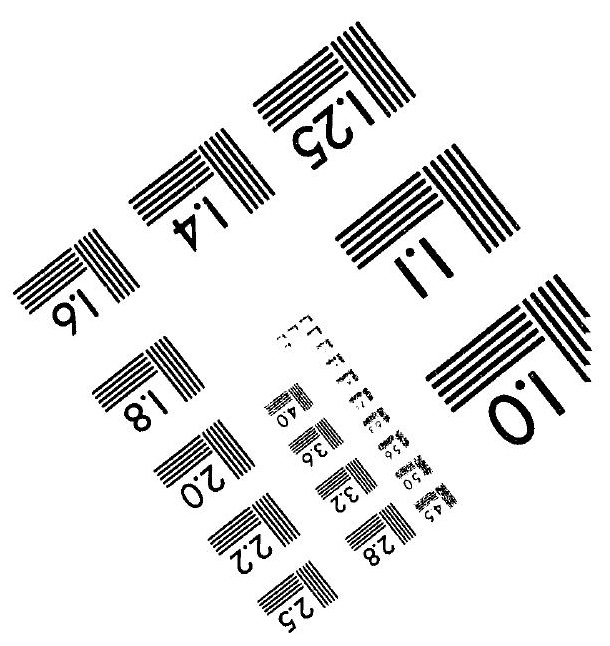



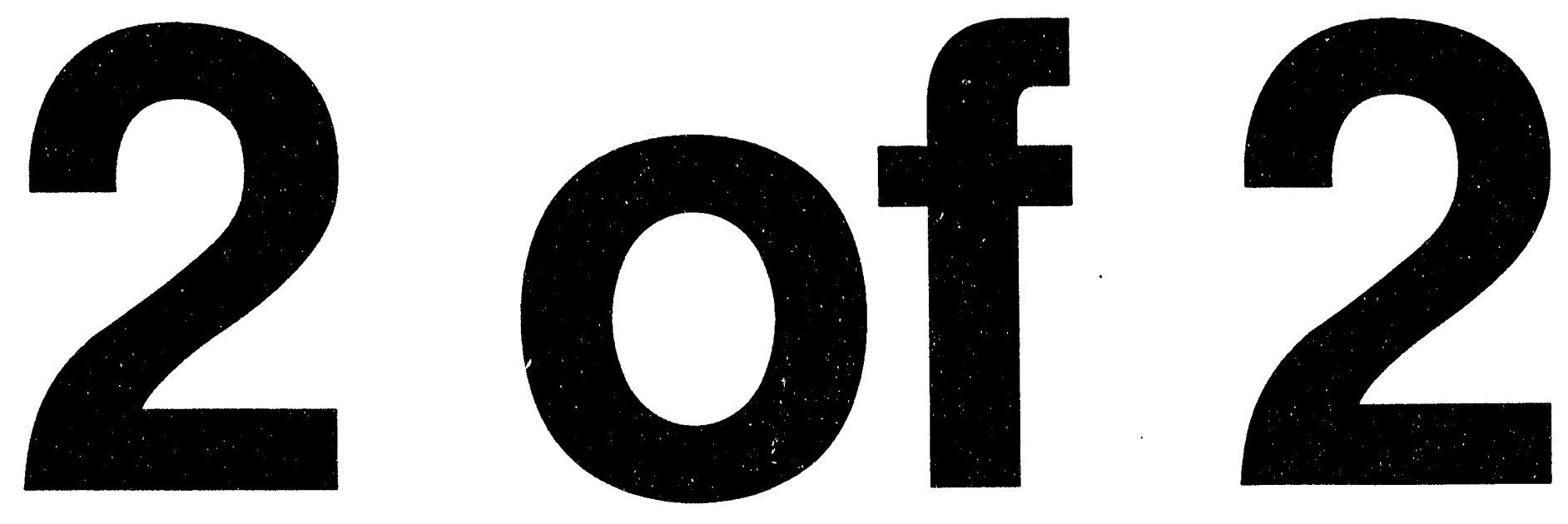
common /fGu/ ifro, deri,pf, ar, dho, rug, dz

common / fplie/ imaxt, epsi, dero

data pee/3.1415926/,rgas/4124.2/

c

character 80 aln

c nnode: number of nodes; iexpl /0,1/ No, Yes explicit

$c$ ifri.ne.1 : switch off friction factor

c itmax: max iters, norif: number of orifices, iditus/0,1/ no Dittus

c( means correction for Twall/Tbulk in Nussel correlation), 1 Yes Dittus c nfu: number of nodes inside fuel

c

itry $=0$

iexplo=iexpl

2013 dero=der

ifro $=i$ iri

der $i=$ der

dho $=d * 0.01$

rug $=r u$

$i \operatorname{maxt}=i t \max$

c eps $i=e p s$

c pin(MPa), tin( $K)$ inlet conditions

$c \mathrm{~d}(\mathrm{~cm}), \mathrm{ru}$ : channel diameter and rugosity

c $x i / 0 ., 1 . / x i=0$. fully explicit; $x i=1$. fully implicit

c der: factor to multiply variable to compute derivative numerically

c typical value 1.01; eps: tolerance to finish iterations; Powt(Mw)

c total power; $r j s(\mathrm{Kw} / \mathrm{cm} 2)$ scale for plot heat rate

c

c

c flowt $(\mathrm{kg} / \mathrm{sec})$ : Total flow

c flow (g/sec) : Channel flow

c

flow $=1000 . *$ flowt $/$ norif

Vamix $=$ Vact*fe

c

c Vact:Volume of fuel elements; Vamix: volume occupied by UC-zrc $\mathrm{mix}$ in

c graphite matrix; exclude coolant holes and clads

c GU235 grams of 0235 in active mix Volume Vamix(Liters) $U$ is

c Enriched ( $2 t$ fraction), PUC mole fraction of UC in UC-zrC $\mathrm{mix}$

$c$ RoUC, RozrC and Rograf $(\mathrm{g} / \mathrm{cm} 3)$ densities of UC, 2 rc and Graphite

C dclad $(\mathrm{cm})$ : clad thickness

c

$\operatorname{RaUC}=(1 .+(1 . / \mathrm{Enr}-1) * 1.012798+.0.05110173 /$ Enr $) * \mathrm{GU} 235 /(1000$. *amix *)

RaZrC $=(1 . /$ PUC -1.$) *$ RaUC*0.4178467

$x U C=R a U C / R O U C$

xzrC=RaZrC/ROZrC

$x G r a f=1 .-x U C-x 2 r C$

RaGraf $=x G r a f \star R o G r a f$

c

Ra=RaGraf+RaZrC+RaUC

npu $=$ nnodo 1

c Hact $(\mathrm{cm})$ :active length; Pfact:Radial Peaking factor at $r$ position

c assume cos in $z$ and $\operatorname{Daxi}(\mathrm{cm})$ axial extrapol.

dzet $=$ Hact $/$ nnodo

roav $=$ Powt * $f$ act /Vamix

C Pfraz: Power factor radial-azimuthal

Pfraz=1.

if(iopth.gt.2) Pfraz=Pfacr*Pfacaz

c

recompute flow according to option

if (iopth.eq.4) flow=flow*Pfraz

Hext $=$ Hact +2 . *Daxi

we=Hext $/$ Hact

Page A-31 


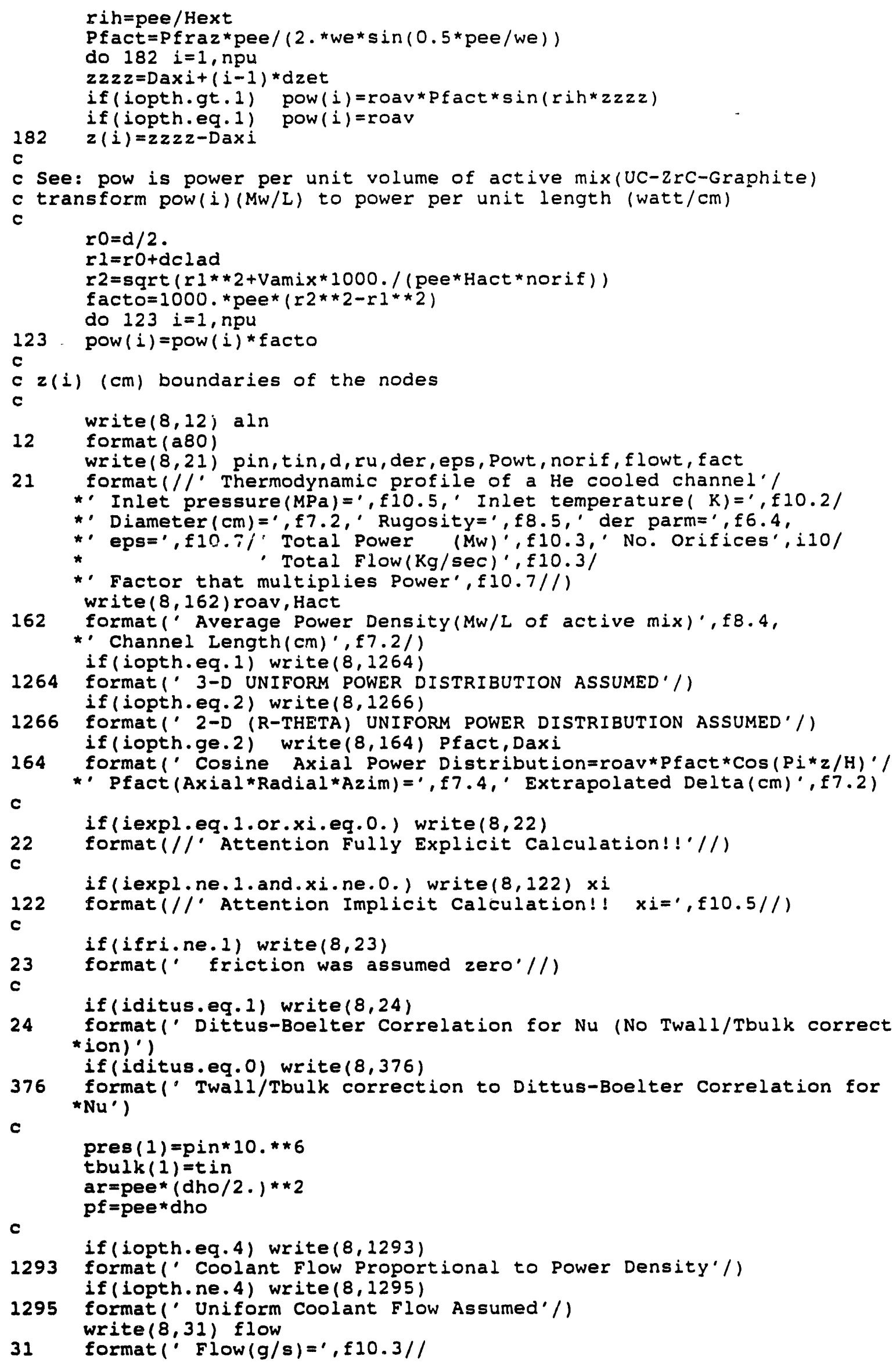




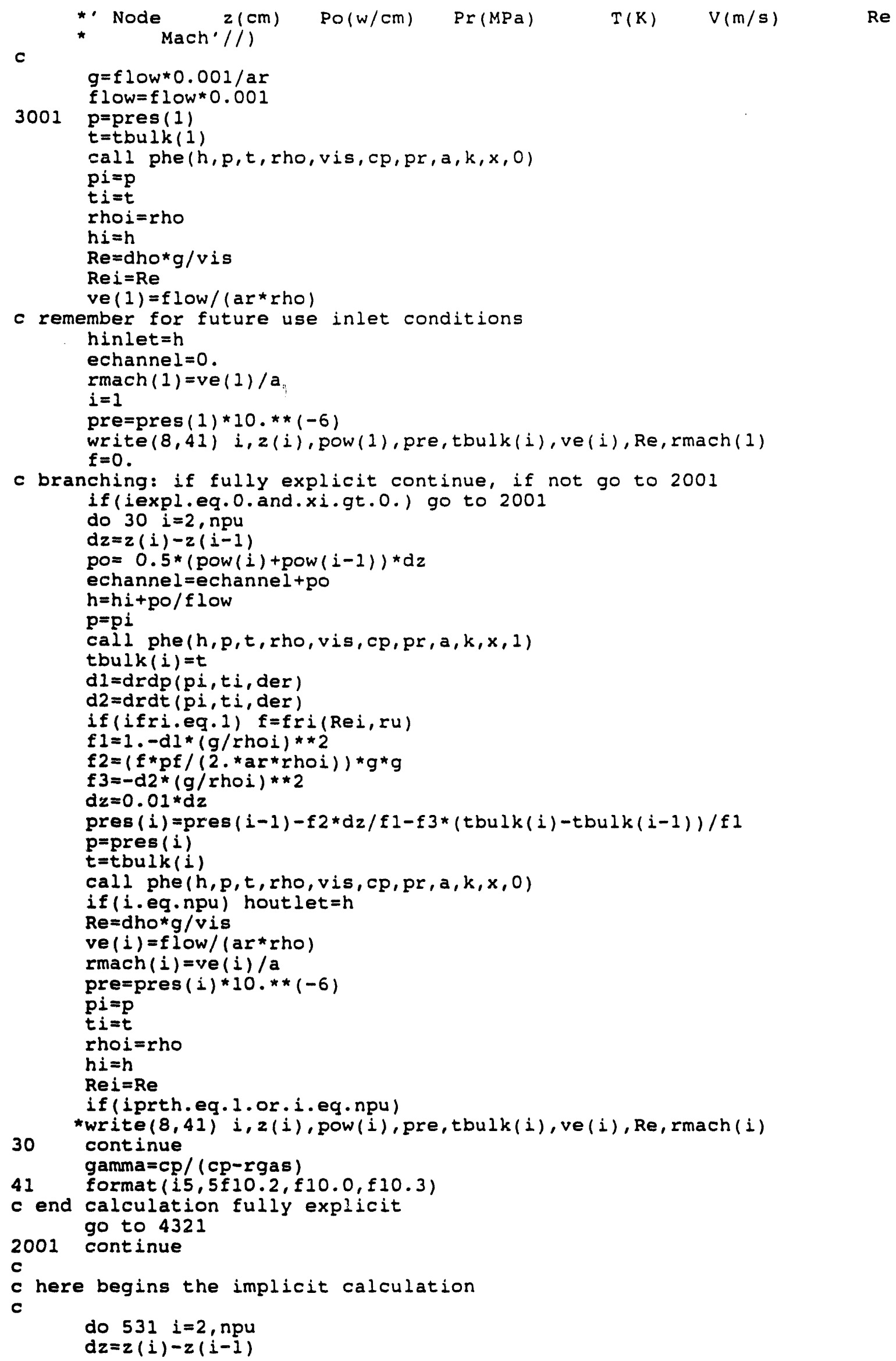




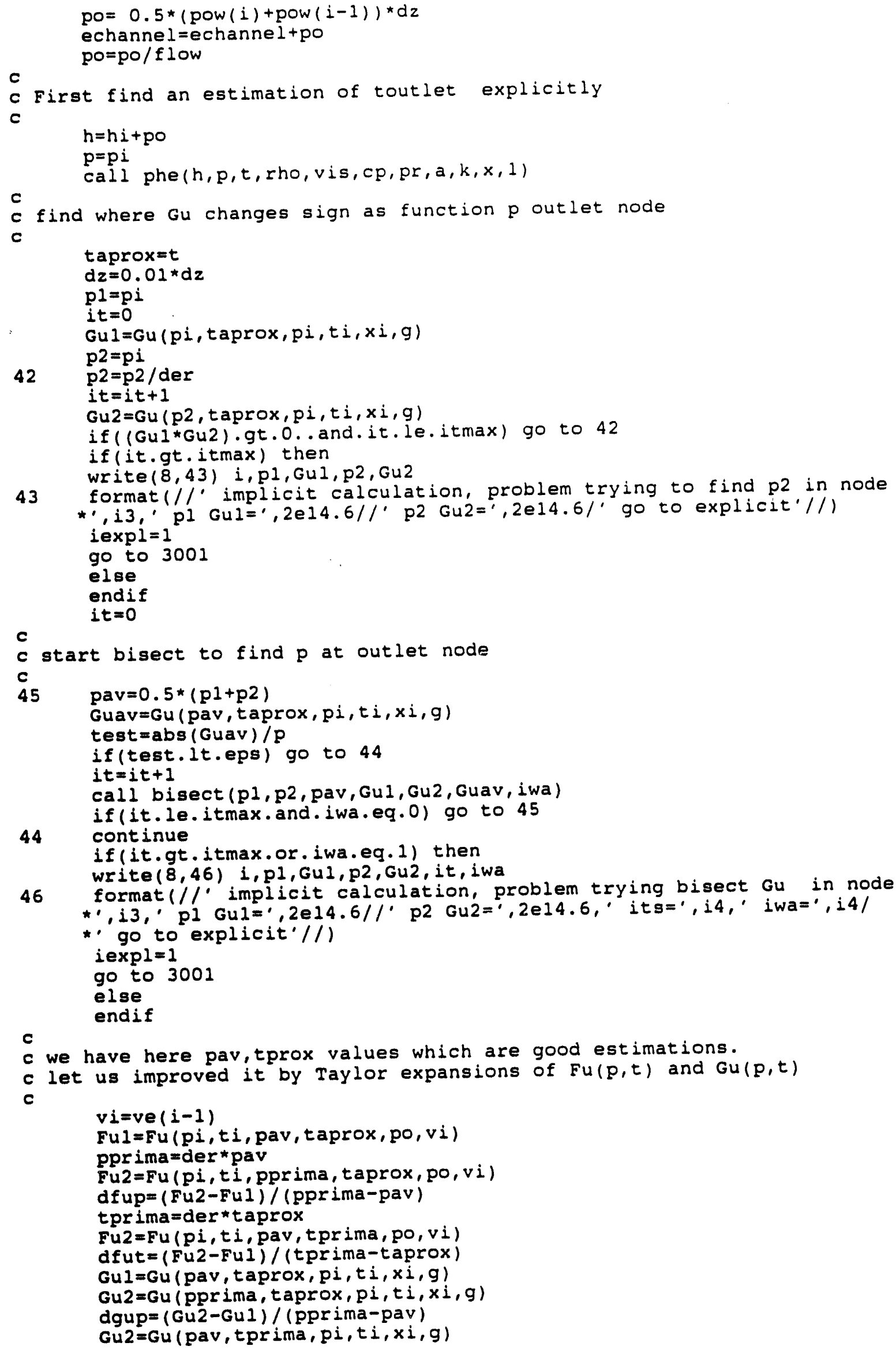




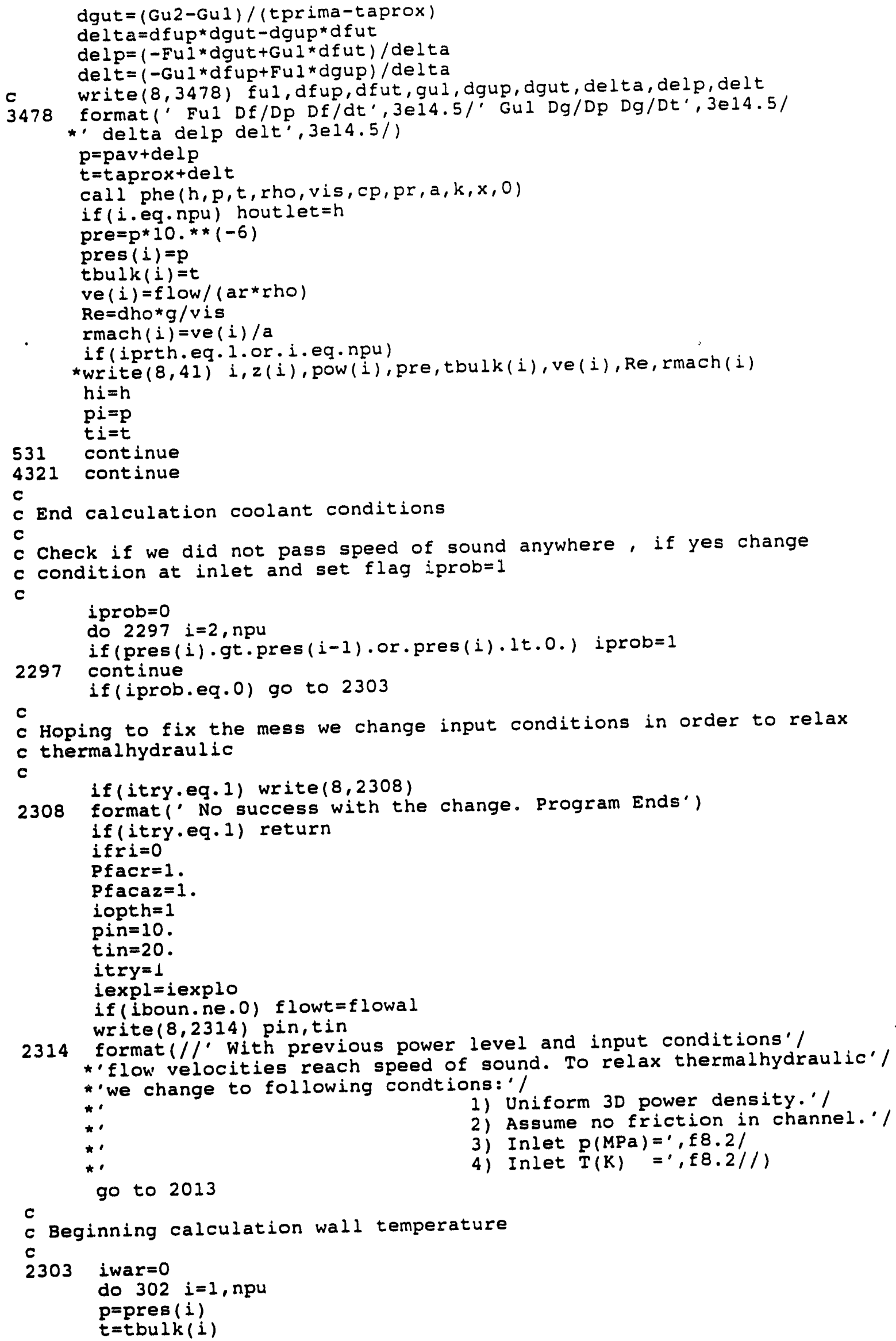


$t \dot{i}=t$

call phe ( $h, p, t, r h o, v i s, c p, p r, a, k, x, 0)$

Rei $=d h o * g / v i s$

Pri $i=$ r

Rki $=k /$ dho

$h=h+0.5 *$ ve (i) $* * 2$

call phe ( $h, p, t$, rho, vis, cp, pr, $a, k, x, 1)$

$\mathrm{T} f \mathrm{~s}=t$

C Apply recovery factor (RC) to stagnation temperature $T f s$ $\operatorname{Rc}=(\operatorname{Pr} i) * * 0.33333333$ Tfa $=t i+R c *(T \in s-t i)$

C First estimation wall temperature

$2 r=z(i) /(100$. *dho)

RNus=RNu (Rei, Pri, 1., Zr)

hCO $=$ RNus*Rki

$r j q=$ pow $(i) * 100 . /($ pee* dhه)

$r j(i)=r j q * 10 * *(-7)$

Tw $1=$ Tfatrjq/hco

$T w(i)=T w 1$

if (iditus.eq.1) go to 3121

c write $(8,143)$ Twl, rjq, hco,Rc, Tfa, Tfs

$i t=0$

$\operatorname{Tr} 1=\operatorname{Tw} 1 / t i$

Fql=Fq(rjq, Zr, Rei, Pri,Rki, Tfa, Trl, Twl)

write $(8,144) \mathrm{TWI}, \mathrm{Fql}$

$T W 2=T w 1$

$422 T w 2=T w 2 *$ der

$\operatorname{Tr} 2=T w 2 / t i$

$i t=i t+1$

Fq2 $=F q(r j q$, Zr, Rei, Pri, Rki, Tfa, Tr2, Tw2)

write $(8,144)$ Tw2, Fq2

if ( $(F q 1 * F q 2) \cdot g t .0$.. and.it. le.itmax) go to 422

if (it.gt.itmax) then

write $(8,431)$ i, Tw1, Fq1, Tw2, Fq2

143 formatl' first estimation, Twl=', f10.3,' Rjq hc',2e14.5/

* Rc Tfa Tfs', $3 f 14.3 / 12 x$, 'Tw', $12 x$, ' $^{\prime} \mathrm{Fq}^{\prime}$ )

144 format (2e14.5)

431 format $/ / /^{\prime}$ implicit calculation, problem trying to find Tw2in node *'13, 'Tw1 Fq1=',2e14.6//'Tw2 Fq2=",2e14.6/" bypass wall '//)

461 format (// implicit calculation, problem trying bisect Fq in node *', i3, 'Tw1 Fq1=', 2e14.6//'Tw2 Fq2=',2e14.6,' its=', i4,' iwa=', i4/ * by pass wall $/ / /$ )

iditus $=1$

iwar $=1$

go to 3121

else

endif

$i t=0$

c start bisect to find $T$ wall

c

$452 T w a=0.5 *(T w 1+T w 2)$

Tra $=$ Twa $/ t i$

Fqav $=F q(r j q$, zr, Rei, Pri, Rki, Tfa, Tra, Twa)

test $=a b s$ (Fqav) /rja

if(test.lt.eps) go to 442

$i t=i t+1$

cal1 bisect ( Tw1, Tw2, Twa, Fq1, Fq2, Fqav, iwa)

if (it. le.itmax. and.iwa.eq.o) go to 452

442

continue

if (it.gt.itmax.or.iwa.eq.1) then

write $(8,461) i, T w 1, F q 1, T w 2, F q 2$, it, iwa

iditus $=1$

iwar $=1$

go to 3121

else

endif

Page A-36 
$T w(i)=T w a$

c From Twa to Tfuel

C

3121 continue

if(xGraf.lt.0.) go to 302

cclad=RkzrC (Twa)

$r j c=r j q * 10 . * *(-4)$

Poa $=r j c * 2 . * r O / r 2 \star \star 2$

$\mathrm{Qh}=\mathrm{Poa} /(1,-(\mathrm{r} 1 / \mathrm{r} 2) \star \star 2)$

c see only one node for the clad

$T c l a d(i)=T w(i)+r j c * r 0 * a l o g(r l / r 0) / c c l a d$

culo $=1$ + + dclad $/$ ro

$\operatorname{Tclaa}(i)=\mathrm{Tw}(i)+r j c * r 0 * \star 2 *(c u l o * a \log (c u l o)-(c u l o-1)) /.\left(\mathrm{dclad} c^{\circ c l a d}\right)$

$\mathrm{d} r=(r 2-r 1) / \mathrm{nfu}$

$\operatorname{Tin} 1=\operatorname{Tclad}(i)$

rinl $=r 1$

$\mathrm{T} f \mathrm{a}=0$.

do 3021 in $=1, n f u$

Cfuel $=\left(\operatorname{RaUC} \operatorname{RkUC}(\operatorname{Tin} 1)+\operatorname{RaZrC}{ }^{*} \operatorname{RkZrC}(\operatorname{Tinl})+\operatorname{RaGraf} \operatorname{RkGraf}(\operatorname{Tin} 1)\right) / \mathrm{R}$

$\star a$

rout $=r i n l+d r$

Tout $=$ Tinl $+(0.25 * Q h * r i n l * * 2 /$ cfuel $) *(2 * *(r 2 / r i n l) * \star 2 * a \log ($ rout $/ r$

*inl $)+1 .-($ rout $/$ rinl $) * * 2)$

Tfa $=$ Tfa+(Tout*rout + Tinl*rinl) $* d r$

Tinl=Tout

rinl=rout

3021 continue

Tfuel (i) = Tout

$\operatorname{Tfua}(i)=\operatorname{Tfa} /(\mathrm{r} 2 * \star 2-r l * * 2)$

302

continue

write $(8,978)$ ro, $51, \dot{5} 2, R a$, RaUC, xUC, RaZrC, xZrC, RaGraf, xGraf, nfu

*, GU235, Vact

978 format $/ / /$ From the coolant Channel to the Uranium Carbide'/

*'Radius(cm) Orifices, Clad, Cell', 3f10.4/

* Averages Densities (g/cm3)',10x,' Volumetric Fraction'/

*

*

Total', f10.4/

UC', 2 f $10.4 /$

ZrC', 2 f $10.4 /$

*' Graphite',2f10.4//' Number of fuel nodes', i4/

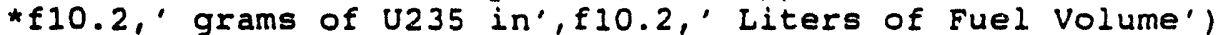

if (iwar.eq.1) write $(8,427$ )

427 format///Due to iterations difficulties we switch to Dittus Correl *ation'

write $(8,976)$

976 format $(/ / /)^{\prime}$

* Tfuel (K)

Node $\quad z(\mathrm{~cm}) \mathrm{J}(\mathrm{KW} / \mathrm{cm} 2)$

Twall $(K)$

Tclad(K) TcladAv

$i=1$

write $(8,977) i, z(i), \operatorname{rj}(i), \operatorname{tw}(i), \operatorname{Tclad}(i), \operatorname{Tclaa}(i), \operatorname{Tfuel}(i), \operatorname{Tfua}(i)$

do $1506 i=2$, npu

if (iprth.eq.1.or.i.eq.npu)

*write $(8,977) i, z(i), \operatorname{rj}(i), \operatorname{tw}(i), \operatorname{Tclad}(i), \operatorname{Tclaa}(i), \operatorname{Tfuel}(i), \operatorname{Tfua}(i)$

1506 continue

977 format $(i 5, f 10.2, f 10.5,5 f 10.2)$

c calculation of axial averages

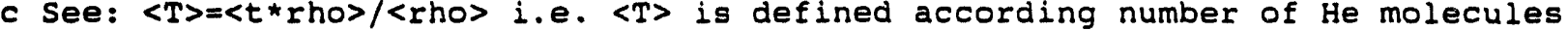

c

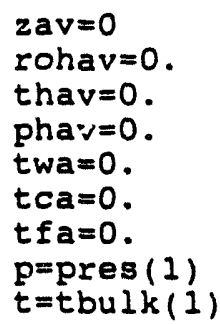


call phe ( $h, p, t, r h o, v i s, c p, p r, a, k, x, 0)$

roin $=$ rho

do $1436 i=2$, npu

$d z=z(i)-z(i-1)$

$z a v=z a v+d z$

phav $=$ phav $+0.5 *(\operatorname{pres}(i)+\operatorname{pres}(i-1)) \star d z$

$t w a=t w a+0.5 *(t w(i)+t w(i-1)) * d z$

$t c a=t c a+0.5 *(t c l a a(i)+t c l a a(i-1)) * d z$

$t f a=t f a+0.5 *(\operatorname{Tfua}(i)+\operatorname{Tfua}(i-1)) * d z$

$p=p r e s(i)$

$t=t b u 1 k(i)$

call phe ( $h, p, t$, rho, vis, cp, pr, $a, k, x, 0)$

roout =rino

rohav $=$ rohav $+0.5 *($ roin + roout $) * d z$

thav $=t h a v+0.5 *(t b u l k(i) *$ roout + tbulk $1 i-1) *$ roin $) * d z$

1436

roin=roout

rohav $=0.001 *$ rohav $/ z a v$

thav =thav/zav

thav $=$ thav $* 0.001 /$ rohav

phav =phav / zav

twa $=t w a / z a v$

$t c a=t c a / 2 a v$

$t f a=t f a / z a v$

$p=$ phav

t=thav

call phe ( $h, p, t$, rho, vis, cp, pr, $a, k, x, 0)$

roav $=r h o * 0.001$

pre $=$ phav $* 0.000001$

1486

Write $(8,1486)$ pre, thav, roav, rohav, twa, tca, tfa

Format (//. Axial Average Conditions in the channel'/

* Coolant Pressure (MPa)', f8.3,' Temperature(K)', f9.3/' Give Den

$* \operatorname{sity}(\mathrm{g} / \mathrm{cm} 3)^{\prime}$, f $10.7 /^{\prime}$

Average Density', f10.7/

*' Wall Temperature $(K)^{\prime}, \pm 9.3 /$

* Clad Temperature (K)', $19.3 /$

* Fuel Temperature $(\mathrm{K})^{\prime}, \mathrm{E9} .3$ )

Tcrit $=$ Tmelt (PUC)

Write $(8,384)$ PUC, Tcrit

384

*ature $(K) ?, f 10.2$ )

$\mathrm{dh}=$ houtlet-hinlet

$d q=e c h a n n e l / f l o w$

dkin $=0.5 *$ (ve (npu) $* * 2-v e(1) * \star 2)$

rel $=\mathrm{dkin} / \mathrm{dg}$

bal $=(d h+d k i n) / d q$

write $(8,987) \mathrm{dq}, \mathrm{dh}, \mathrm{dkin}$, rel, bal

987 format (//' HEAT BALANCE FOR THE CHANNEL'//

* 'dQ: heat to coolant (joule/kg)',el4.5/'dH: change of enthalpy

1 (joule/kg)',e14.5/'dKin change kinetic energy (joule/kg)', el4.5/

c

$2^{\prime}$ relative dKin/dQ', $f 10.6 /{ }^{\prime}$ Balance $(\mathrm{dH}+\mathrm{dKin}) / \mathrm{dQ} \mathrm{Q}^{\prime}, \mathrm{f} 14.7$ )

return

end

c friction factor for one phase flow

function fri(Re, ru)

c where Re is the Reynold's number and ru=e/d (rugosity)

c colebrook-White correlation, page 115 ANS book about Gas cooled Rs.

if (Re.1e.2000.) then

fri $i=16$. $/$ Re

else

fri $i=0.001375 *(1 .+(20000 . * r u+1000000 . / \operatorname{Re}) * 0.33333333)$

endif

return

end

c calculate drho/dp 


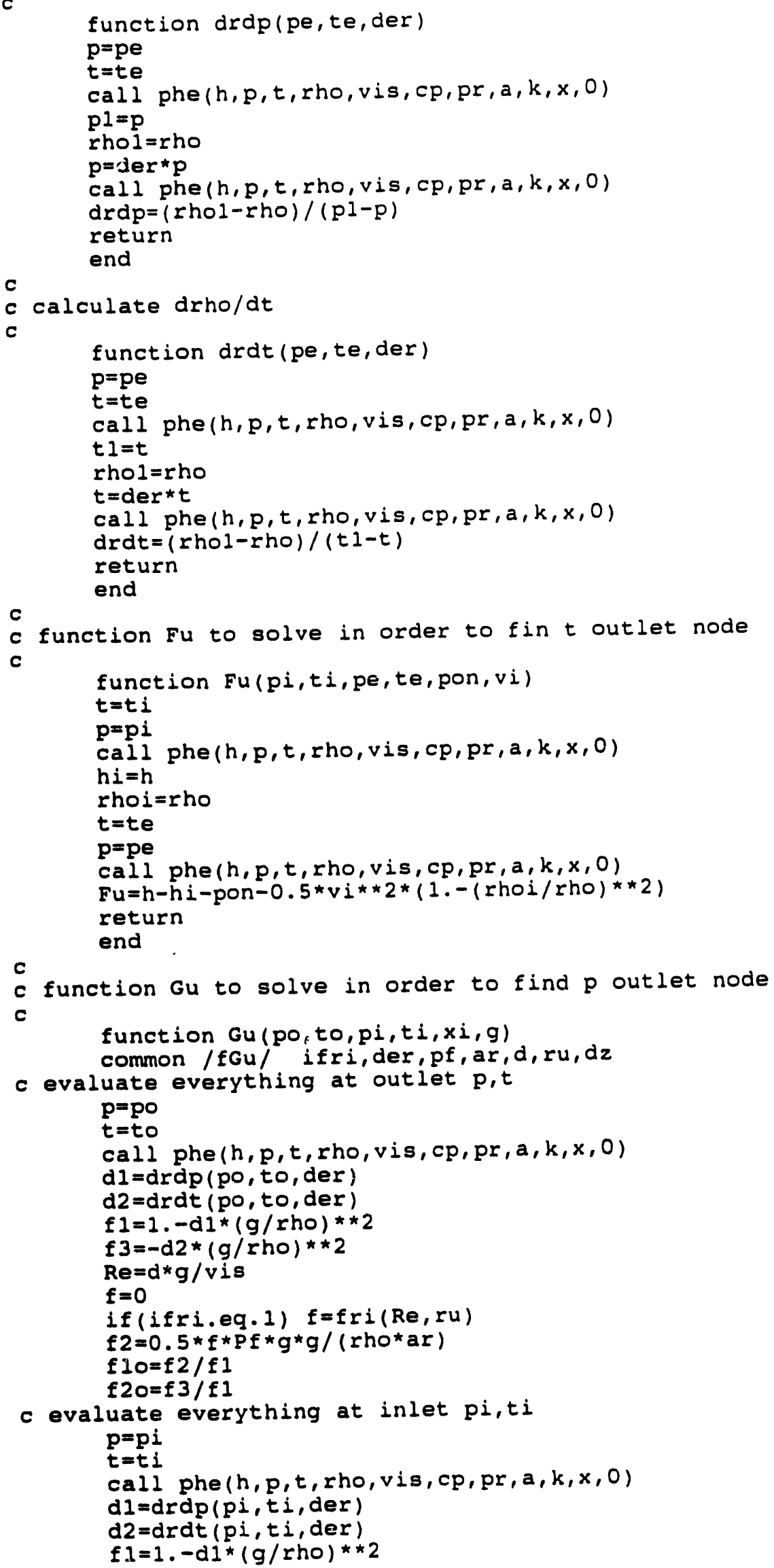




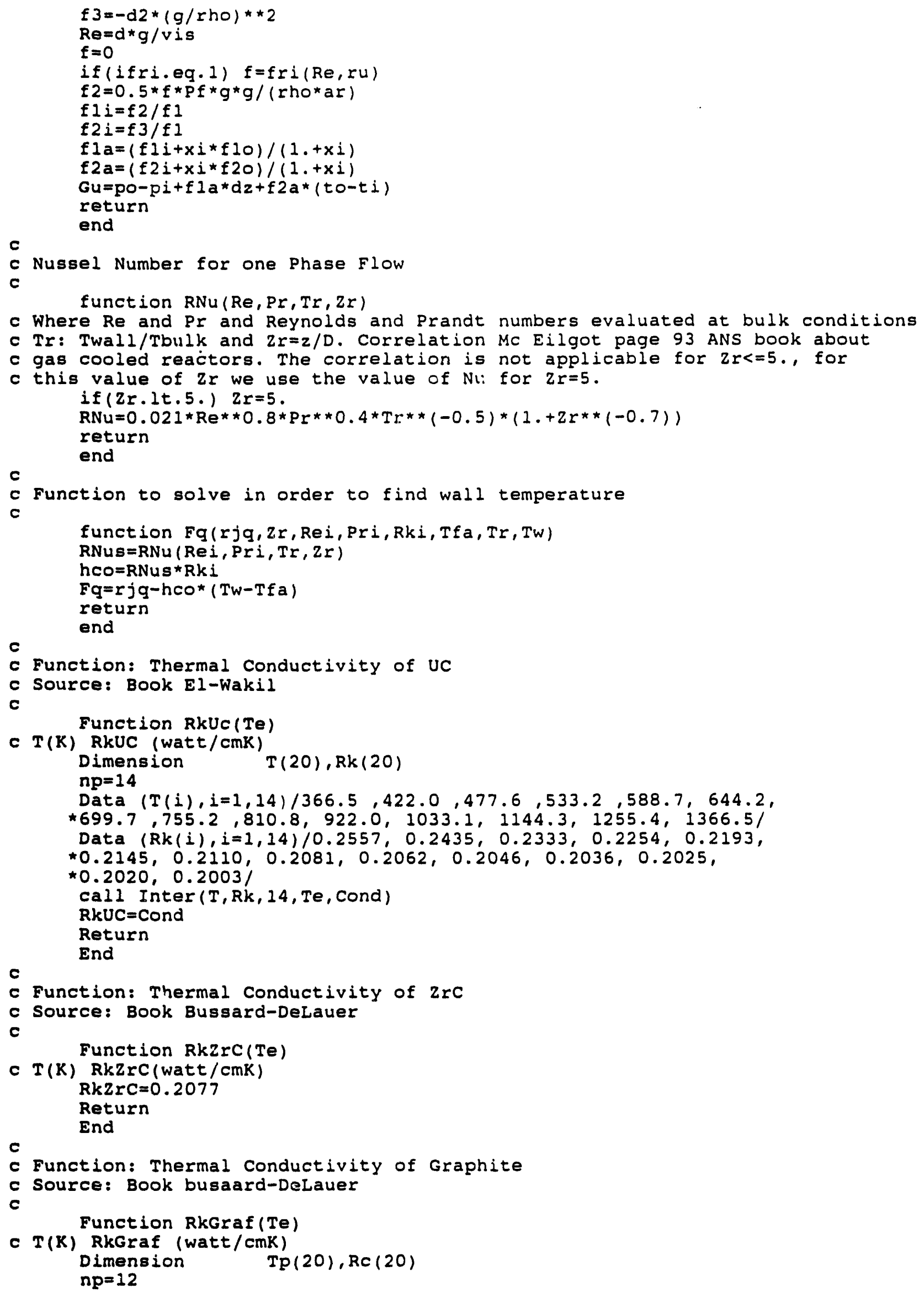


Data $(\operatorname{TP}(i), i=1,12) / 277.8,416.7,555.5,833.3,1111.1,1388.9$,

$\star 1666.7,1944.4,2222.2,2500 ., 2777.8,3333.31$

Data $(R C(i), i=1,12) / 1.743,1.414,1.097,0.817,0.6339,0.5485$,

$\star 0.4632,0.4145,0.3657,0.3413,0.3169,0.2926 /$

call Inter ( $T p, R c, n p, T e$, cond)

RkGraf $=$ Cond

Return

End

C

c Function: Melting Temperature of Uc-zrc mix

c Source: Book busaard-DeLauer

c

Function Tmelt (PUC)

c Tmelt(K), PUc mol fraction of Uc in UC-zrc mix Dimension xmol (20), T(20)

$n p=6$

Data $(x \operatorname{mol}(i), i=1,6) / 0.0,0.2,0.4,0.6,0.8,1.0 /$

Data $(t(i), i=1,6) / 3758,, 3594 \ldots, 3437$, 3250, , 3022., 2687. /

call Inter ( $x$ mol, $T, n p, P U C, T m)$

Tmelt $=\mathrm{Tm}$

Return

End

$c$

c Properties of He

subroutine phe ( $h, p, t$, rho, vis, $c p, p r, a, r k, x$, iflag)

common / fphe/ nit, eps, der

c Thermodynamic and Transport Properties of Helium

C from Appendix B, page 405, Thermal and Flow Design of Helium-Cooled

c Reactors by G. Melese and R. Katz American Nuclear Society, 1984. $\mathrm{cp}=5193$.

$R=2077.22$

$x=1$.

$c$

c go to 10 if iflag=1 (ie input is $p, h$ )

c

if (iflag.ne.0) go to 10

$1 \quad r h o=p /(R * t+p * b(t))$

$h=c p * t+(b(t)-t * c(t)) * p$

$z=1 .+p * b(t) /(R * t)$

$a=z * \operatorname{sqrt}(1.666 * R * t)$

vis $=3.953 \mathrm{e}-7 * t * * 0.687$

$r k=2.774 e-3 * t * \star 0.701$

$p r=0.740 * t * *(-0.014)$

return

10 continue

$c$ use bisect method to find $t$ from $p$ and $h$

$r c=$ der

$t l=h / c p$

en $1=\operatorname{ent}(h, p, t 1)$

if (enl.1t.0.) $r \mathrm{c}=1 . / \mathrm{rc}$

$t 2=t 1$

it $=0$

30

$t 2=t 2 * r c$

$i t=i t+1$

en2 $=$ ent: $(h, p, t 2)$

if (enl*en2.1t.0) go to 20

if(it.lt.nit) go to 30

write $(6,50) \mathrm{h}, \mathrm{p}$

50 format (//'Program stop at phe(1). Unable to find $T$ for input $h='$, $\star e 14.5$, and $p=1, e 14.5 / / 1$

stop

20 continue

it $=0$

iwa $=0$ 


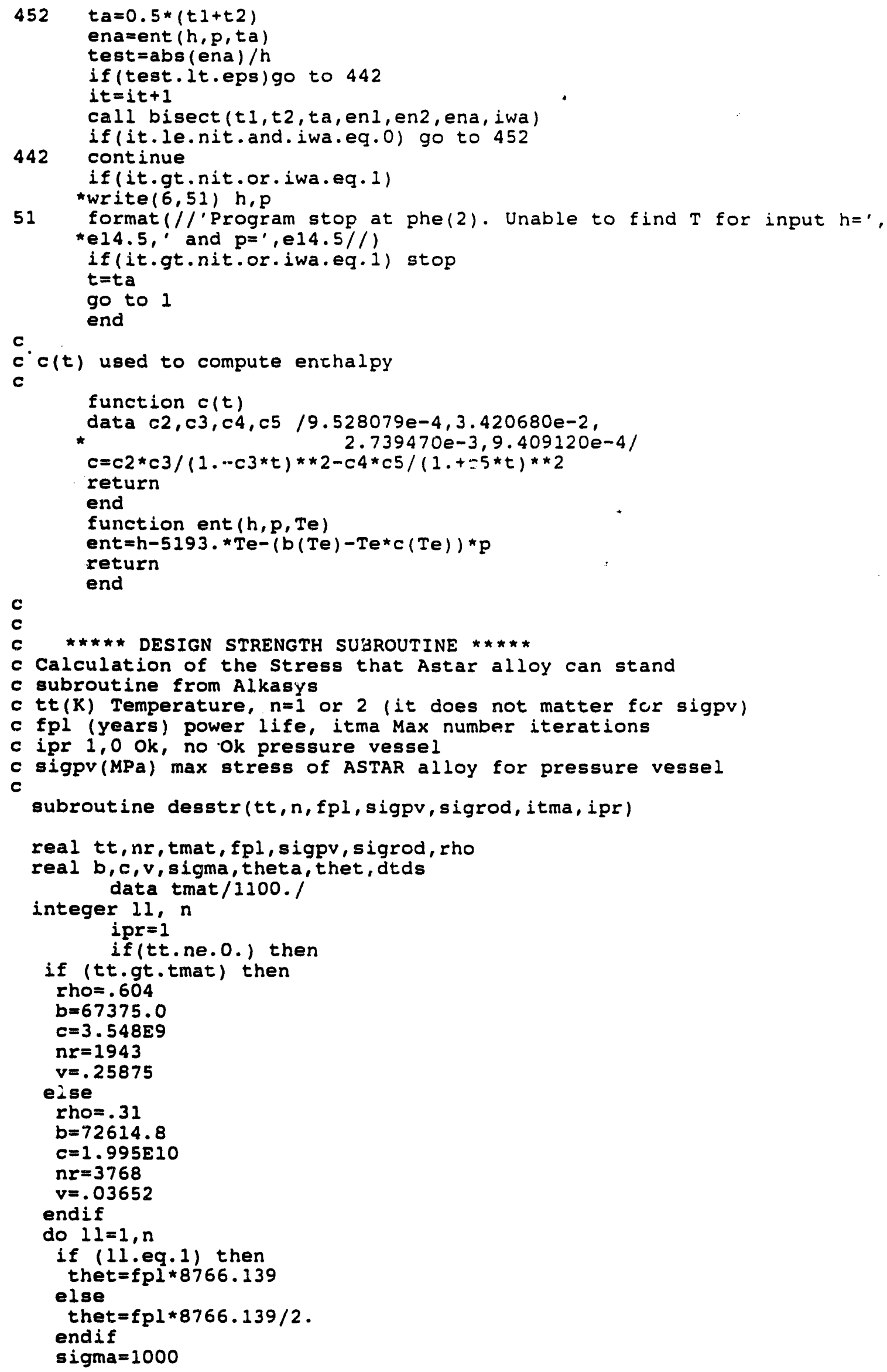




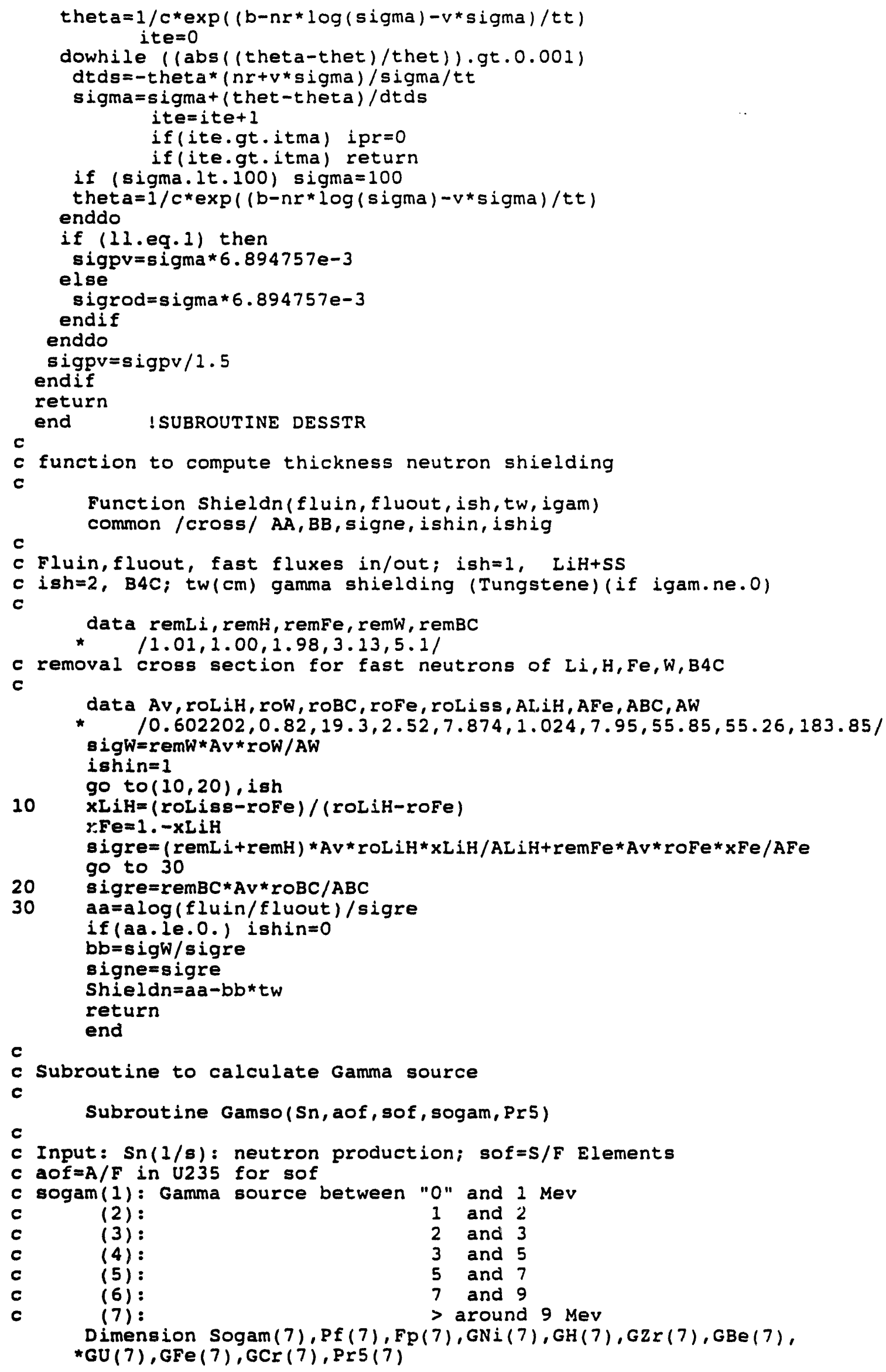


C

Pf: Prompt Fission Gammas; Fp: Fission Products Gammas(Both, Per Fiss) GNi to GCr:Captures Gammas (per Event) for indicated Elements Ni,..Cr

C

c

c See: Total number of Absorptions= Sn(Total production)

$\operatorname{Data}(\operatorname{Pf}(i), i=1,7) / 3.1,2.11, .73, .26, .0428,0 ., 0 . /$

$\operatorname{Data}(\operatorname{Fp}(i), i=1,7) / 9.03,1.137, .4,0,0 ., 0 ., 0 . /$

$\operatorname{Data}(G N i(i), i=1,7) / .84, .40, .23, .23, .34, .62,0 . /$

$\operatorname{Data}(\mathrm{GH}(i), i=1,7) / 0,0 ., 1,0,0,0,0.1$

$\operatorname{Data}(\operatorname{Gz}(i), i=1,7) / 0 ., 0 ., 0 ., 1.13, .35, .04,0 . /$

Data (GBe (i), $i=1,7) / 0,0,0 ., .54, .73, .0, .0 /$

$\operatorname{Data}(G U(i), i=1,7) / 2.54,1.78, .91, .34, .0, .0,0.041 /$

Data (GFe (i), $i=1,7) / .75, .60, .27, .23, .25, .38, .02 /$

$\operatorname{Data}(\operatorname{GCr}(i), i=1,7) / .85, .41, .21, .12, .23, .39, .06 /$

Data FNi2, FH2, FZr2, FBe2, FU82, FFe2, FCr2

* /.12,.083,.088,.051,.045,.016,.008/

Data FNiO, FHO, FZrO, FBeO, FU8O, FFeO, FCrO

* $10.0 .0 .076, .087, .069,0.0 .1$

Data Rnu/2.44/

F: total number of fission

A5: Total number of absorptions(capturetfission) in 4235

C5: total number of captures in 4235

ct: " " "captures

$F=S n / R n u$

$A 5=F *$ aof

$C 5=A 5-F$

$\mathrm{C} t=\mathrm{Sn}-\mathrm{F}$

$\mathrm{CNi}=(\mathrm{FNiO}+(\mathrm{FNi} 2-\mathrm{FNiO}) * \operatorname{sof} / 2). * \mathrm{Ct}$

$\mathrm{CH}=(\mathrm{FHO}+(\mathrm{FH} 2-\mathrm{FHO}) *$ sof $/ 2). * \mathrm{Ct}$

CZr $=($ FZrO + (FZr2-FZrO $) * \operatorname{sof} / 2) * C$.

$\mathrm{CBe}=(\mathrm{FBeO}+(\mathrm{FBe} 2-\mathrm{FBeO}) * \operatorname{sof} / 2). * \mathrm{Ct}$

CUB $=($ FU80 $+($ FU82-FU80 $) *$ sof $/ 2) * C$.

$\mathrm{CFe}=(\mathrm{FFeO}+(\mathrm{FFe} 2-\mathrm{FFeO}) *$ sof $/ 2). * \mathrm{Ct}$

CCr $=($ FCrO+(FCr2-FCrO $) * \operatorname{sof} / 2) * C$.

Do $10 i=1,7$

Gam5 $=F^{*}(P f(i)+F p(i))+C 5 * G U(i)$

$\operatorname{sogam}(i)=\mathrm{Gam} 5+\mathrm{CU} 8 * \mathrm{GU}(\mathrm{i})+\mathrm{CN} i \mathrm{GNi}(\mathrm{i})+\mathrm{CH} * \mathrm{GH}(i)$

* $+C Z r * G Z r(i)+C B e * G B e(i)+C F e * G F e(i)+C C r * G C r(i)$

10 $\operatorname{Pr} 5(i)=100 . *$ Gam5/Sogam (i)

continue

return

end

c

Subroutine Shieldga (Sogam, Dose, Dosea, Dosew, tiempo, tgam, ten, igam, *ishn, iten)

Common /CROSs/AA, BBB, signe, ishin, ishig

Shieldga: Computes gamma shielding

Sogam(i), $i=1,7$ Gamma Source (photons/sec); Dose(rad): Integrated

Dose at load area; Dosew(rad): Dose without the shielding

Dorea: dose for tgam

igam: flag if $=1$ problems with iterations

Tgam(cm): Thickness of Tungsten

ten(cm) thickness of $n$ shielding, ishn=1, LiH, ishn=2, B4C

iten/0,1//ten input, ten iterated by this subroutine/

tgam: iterated, if necessary, to have Dose=input

Index (1): Gamma Processes between "O" and 1 Mev

(2):

(3):

(4):

(5):

(6):

(7):

1 and 2

2 and 3

3 and 5

5 and 7

7 and 9

$>$ around $9 \mathrm{MeV}$

common /Gamsh/ coBe, coTa, coC, coZr, coU, coH, coNi, coFe, coCr, coTi, cosi

*,dist, Req, shg (7), shgnb (7), Zeq (7), Dosewg(7), Doseg(7), itmag, epsg

Page A-44 
*, aten, Dosen, Doseng ( 7 )

dimension $\operatorname{Sogam}(7), \mathrm{GBe}(7), \mathrm{GW}(7), \mathrm{GC}(7), \mathrm{GZr}(7), \mathrm{GU}(7), \mathrm{GH}(7), \mathrm{GFe}(7)$, *GTi(7), GSi(7),b(4,7), a $(4,7), a 4(4,7), z b(4), a(4), a 1(4), a 2(4), x h(7)$ $\star, \operatorname{ega}(7), \operatorname{sign}(7), A n(7), A \ln (7), A 2 n(7)$

$c$

data (GBe(i), $i=1,7) / .0773, .0459, .0394, .0266, .0211, .0180, .0161 /$

data (GW $(i), i=1,7) / .125, .0492, .0437, .0402, .0418, .0438, .0465 /$

data (GC(i), $i=1,7) / .087, .0518, .0444, .0304, .0245, .0213, .0194 /$

data $(\operatorname{GZr}(i), i=1,7) / .0851, .0467, .0414, .0349, .0344, .0349, .0359 /$

data $(G U(i), i=1,7) / .176, .0548, .0484, .044, .0455, .0479, .0511 /$

data $(G H(i), i=1,7) / .173, .103, .0876, .0579, .0446, .0371, .0321 /$

data $(G F e(i), i=1,7) / .0828, .0485, .0424, .033, .0304, .0295, .0294 /$

data $(G T i(i), i=1,7) / .0876, .0518, .0451, .0338, .0302, .0285, .0280 /$

C

data (GSi(i), $i=1,7) / .0869, .0517, .0447, .0323, .0277, .0254, .0243 /$

C GBE...GSi are the total mass attenuation coefficients $(\mathrm{cm} * \star 2 / \mathrm{g})$

$c$ for the 7 gamma groups. Fe $(z=26), \mathrm{Ni}(z=28)$ and $\mathrm{Cr}(z=24)$ can be lumped

c together; $\operatorname{Ta}(z=73)$ lumped with $W(z=74) ; G Z r(z=40)$ is indeed GMo $(z=42)$

$c$ and $\operatorname{GTi}(z=22)$ is indeed $\mathrm{GCa}(z=20)$

c Also $L i(z=3)$ and $B(z=5)$ are lumped with $B e(z=4)$

C

$\operatorname{data}(b(1, j), j=1,7) / 8,5.5,4.5,3.8,3.1,2.3,2.25 /$

data $(b(2, j), j=1,7) / 10 ., 8,, 5.5,3.75,2.9,2.35,2.0 /$

data $(b(3, j), j=1,7) / 3.3,2.9,2.7,2.05,1.2, .7, .6 /$

data $(b(4, j), j=1,7) / 1.65,2.45,2.15,1.65, .96, .67, .5 /$

$c$

data $(a 3(1, j), j=1,7) / .11, .082, .074, .066, .064, .062, .060 /$

data $(a 3(2, j), j=1,7) / .0948, .0895, .0788, .075, .0825, .0833, .095 /$

data $(a 3(3, j), j=1,7) / .043, .069, .086, .118, .171, .205, .212 /$

c

data $(a 3(4, j), j=1,7) / .032, .045, .097, .123, .175, .204, .214 /$

data $(a 4(1, j), j=1,7) / .044, .093, .116, .130, .152, .150, .128 /$

data $(a 4(2, j), j=1,7) / .012, .04, .07, .082, .075, .0546, .0116 /$

data $(a 4(3, j), j=1,7) / .148, .188, .134, .070, .0, .052, .144 /$

data $(a 4(4, j), j=1,7) / .296, .178, .103, .064, .059, .067, .08 /$

c

$\operatorname{data}(2 b(i), i=1,4) / 13,, 26 ., 74, .82 . /$

c

$c b(i, j), a 2(i, j)$ and $a 4(i, j)$ are build up parameters for groups $j$

$c$ and atomic number $z=z b(i)$

c

$\operatorname{data}(\operatorname{rh}(i), i=1,7) / 4 \cdot 5 e+5,5.5 e+5,7 . e+5,8 . e+5,8.5 e+5,9 . e+5,1 . e+6 /$

c rh(i) Gamma Flux Mev/cm2sec that produces 1 Roentgen/hour

data $(\operatorname{Ega}(i), i=1,7) / 0.5,1.5,2.5,4,6 ., 8,10.1$

c ega(i) Mev Average Energy of gamma group i

data $A B e, A T a, A W, A C, A Z r, A U, A H, A N i, A F e, A C r, A T i, A S i, A v, R O W$

$\star / 9.0122,180.948,183.85,12.011,91.22,235.044,1.00797,58.71,55.847$,

$\star 51.996,47.90,28.086, .602202,19.3 /$

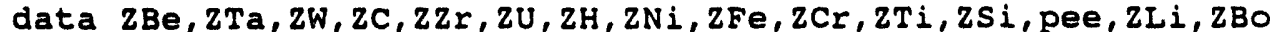

*/4.,73.,74.,6.,40.,92.,1.,28.,26.,24.,22.,14.,3.141593,3.,5./

$c$

data roLiH, rOBC, RosS, RoLis $/ 0.82,2.92,7.874,1.024 /$

igam $=1$

ishig $=1$

$x$ LiH $=($ ROLiS-ROSS) / (ROLiH-ROSS)

$x S S=1,-x L i H$

c Attenuation*Build Up factor for $N$ Shield(which also shield gammas)

c

do $101 j=1,7$

go to $(111,222)$, ishn

c ishn=1 LiH+SS

$1118 i g=\operatorname{ROLiS} *(x L i H * 0.8732 * G B e(j)+x L i H * 0.1268 * G H(j)+x S S * G F e(j))$

Page A-45 


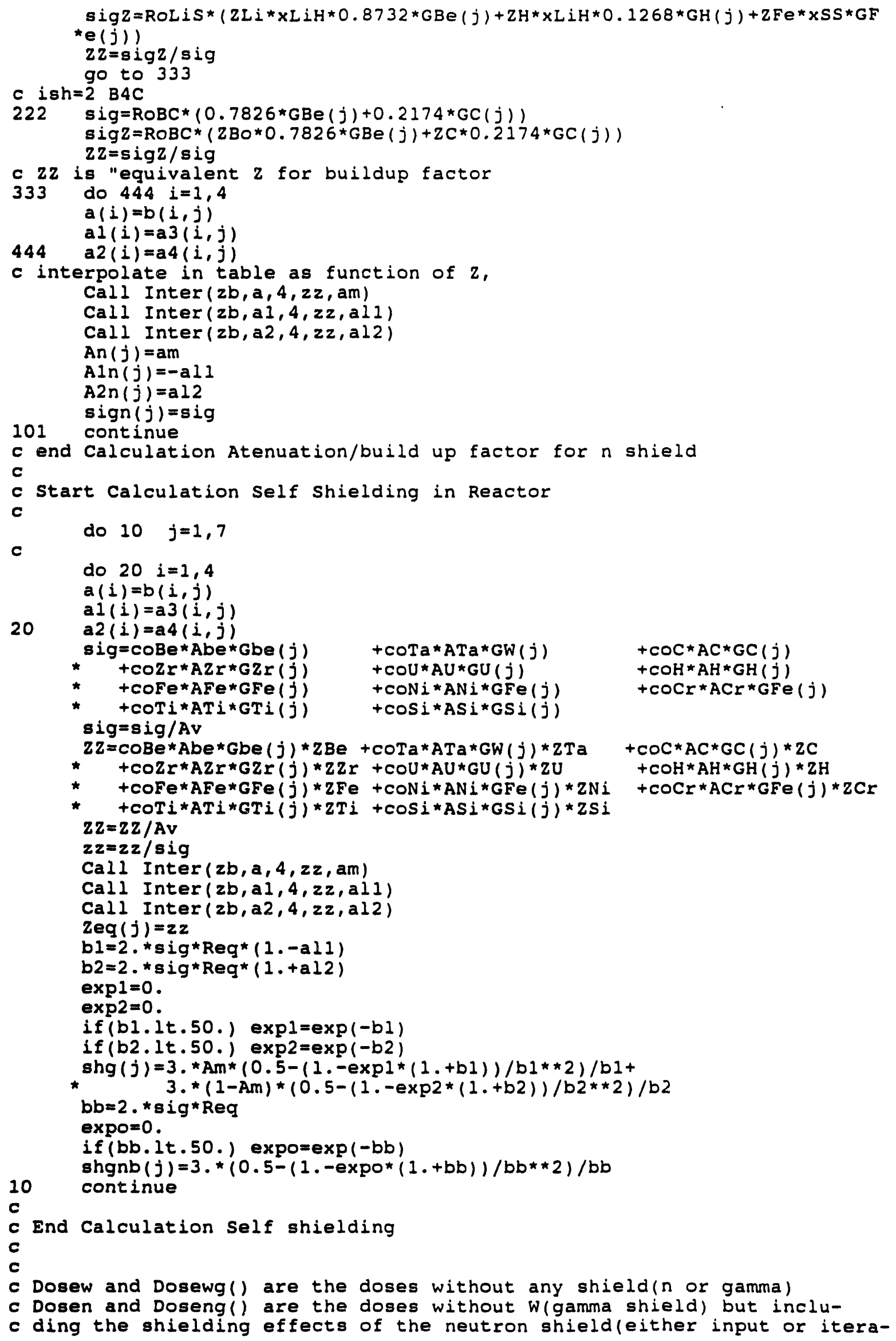


time $=$ tiempo $/ 3600$.

Dosew $=0$.

Dosen $=0$.

if (iten.eq.1) ten $=A A$

if (iten.eq.1.and.ishin.eq.0) ten $=0$.

do $30 \quad i=1,7$

$\operatorname{tsn}=\operatorname{sign}(i) \star \operatorname{ten}$

$\operatorname{shin}=\exp (-t \operatorname{sn}) *(A n(i) * \exp (-t \operatorname{sn} * A 1 n(i))+(1 .-A n(i)) * \exp (-t \operatorname{sn} * A 2 n(i))$

* 1

Dosewg $(i)=t i m e * \operatorname{Sogam}(i) * \operatorname{shg}(i) \star E g a(i) /\left(4{ }^{*}\right.$ pee*dist**2*rh(i))

Doseng $(i)=\operatorname{Sh} i n *$ Dosewg $(i)$

Dosew=Dosew+Dosewg (i)

30

Dosen $=$ Dosen+Doseng $(i)$

if (Dosen. le. Dose) ishig $=0$

if (ishig.eq.0) tgam $=0$.

if (ishig.eq.0) return

C

c.

Return if there is no need for gamma shield (thick $W=0$.)

$t 1=0$.

Dose1=Dosen

f $1=$ Dose1-Dose

c

c Starts iteration on tgam:

c First look for

c $t 2$ such that Dose $(t 2)<$ Dose

c

$i t=0$

sig $=G W(1) *$ RoW

t2=alog (Dosewg (1)/Dose)/sig

51 dose $2=0$.

do $40 \quad i=1,7$

$a m=b(3, i)$

all $=-a 3(3, i)$

a) $2=24(3, i)$

sig $=G W(i) \star R O W$

$B F A=a m * \exp (-a l 1 * s i g * t 2)+(1 .-a m) * \exp (-a 12 * s i g * t 2)$

40

dose $2=$ dose $2+$ Dosewg $(i) * \exp (-s i g * t 2) * B F A$

if (dose2.1t.dose) go to 50

$t 2=1.01 * t 2$

$i t=i t+1$

if(it.le.itmag) go to 51

igam $=0$

write $(8, *)$ 'Problem with Calculation of Gamma Shielding'

50

continue

f2 2 Dose2-Dose

if (igam.eq.0) return

it $=0$

61 tgam $=0.5 *(t 1+t 2)$

dosea $=0$.

do $41 i=1,7$

$a m=b(3, i)$

a) $1=-a 3(3, i)$

a) $2=a 4(3, i)$

sig $=G W(i)$ * ROW

$B F A=a m * \exp (-a 11 * s i g * t g a m)+(1 .-a m) * \exp (-a 12 * s i g * t g a m)$

c

recompute neutron shielding(ten) because $W$ also shield neutrons

$c$ see: if ten<0. program set ten to 0 . (ie no need of $n$ shielding

because $W$ is enough also if ishin=0 (fluin lt prescribed fluout)

if (iten.eg.1) ten=AA-BBB*tgam

if (ten.1t.0.) ten $=0$.

if (iten.eq. 1 . and. ishin.eq.0) ten $=0$.

tsn=sign (i) *ten

$\operatorname{shin}=\exp (-\operatorname{tsn}) *(A n(i) * \exp (-t \operatorname{ss} \star A 1 n(i))+(1 .-A n(i)) * \exp (-t \operatorname{sn} \star A 2 n(i))$ 


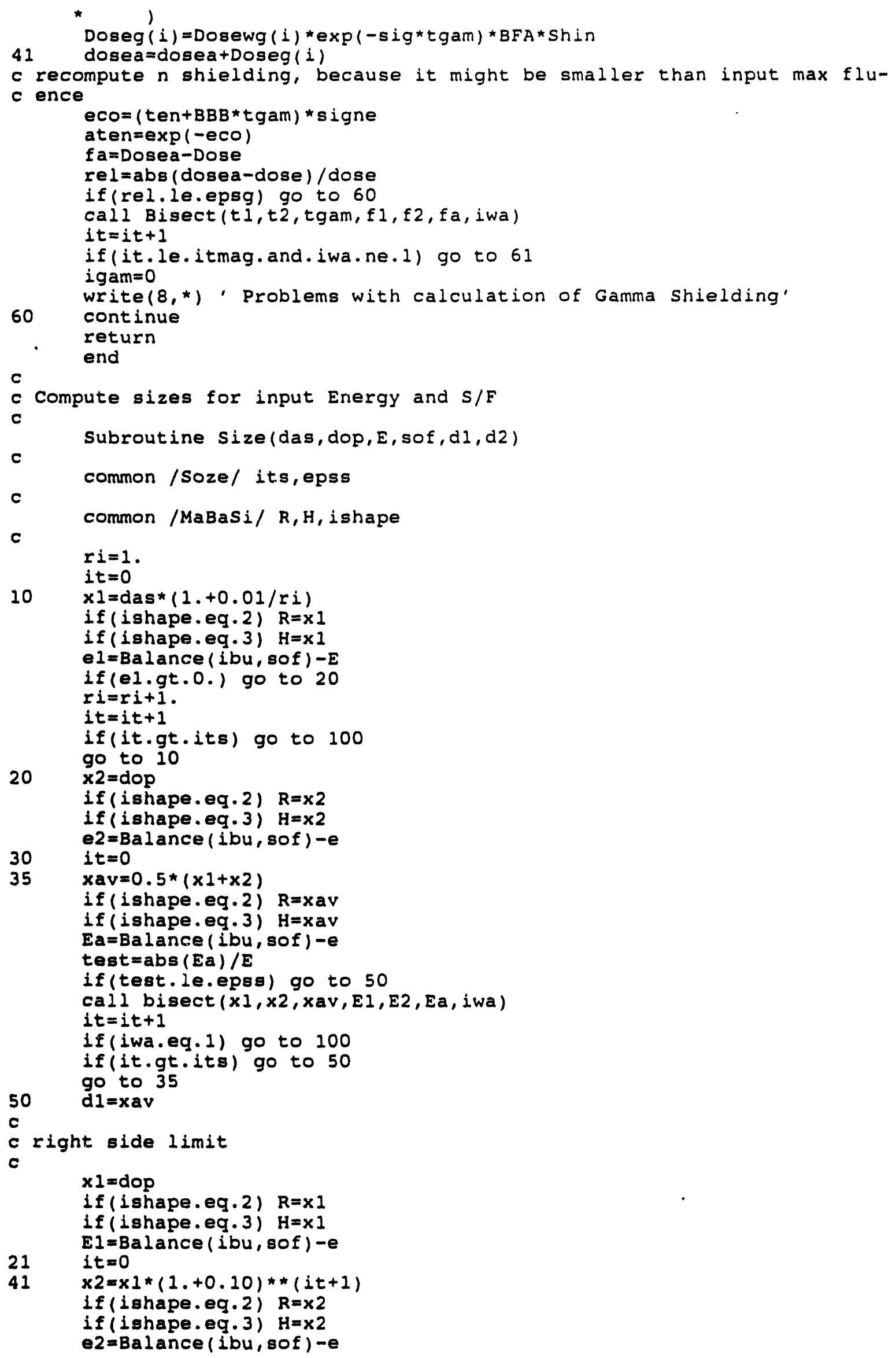


if (e2.gt.o.) go to 31

it $=i t+1$

if (it.gt.its) go to 100

31
36

go to 41

it $=0$

$x a v=0.5 *(x 1+x 2)$

if (ishape.eq.2) $R=x a v$

if (ishape.eq. 3) $\mathrm{H}=\mathrm{xav}$

$E a=B a l$ ance (ibu, sof) -e

test $=a b s(E a) / E$

if (test.le.epss) go to 51

call bisect $(x 1, x 2, x a v, E 1, E 2, E a, i w a)$

$i t=i t+1$

if(it.gt.its.or.iwa.eg.1) go to 100

go to 36

$51 \quad \mathrm{~d} 2=x a v$

return

100 write $(8,101)$ it

101 format (//' Program stop at subroutine size'/' iterations=', i10/) stop

end 


\section{APPENDIX B. LISTING OF CODE NEPPIN}

c program to estimate preliminary design of fuel pin, liquid li reactor c for space applications.

c Assumptions

c 1) fuel cell: liquid Li(coolant), Astar alloy clad, W liner, He gap and UN pellet.

2) Astar pressure vessel, OBe reflector and $B 4 C(908 B 10)$ drums in reflector.

3) Gamma Shield: W; Neutron Shield:B4C or LiH in SS matrix

4) Data base parameters: Enrichment and pitch/drod for drod $=6.4 \mathrm{~mm}$

c character 80 tit, aldum

c dimension aux (5), bux (5), wbeth (8), th(8), pfr $(3,3)$, U5md(5), isha (5), $\star \operatorname{pfrm}(3,3), \operatorname{Sogam}(7), \operatorname{PrU}(7), \operatorname{Emin}(5,5), \operatorname{Ras}(5,5), \operatorname{Has}(5,5), \operatorname{Rop}(5,5)$ $\star$, Hop $(5,5), \operatorname{enri}(5), \operatorname{podi}(5), \operatorname{BUal}(5), \operatorname{Rd}(5), \operatorname{Hd}(5), \mathrm{dpvd}(5), \operatorname{del} \mathrm{p}(5)$, *GRea (5), dkbup (5), dkpvd (5), rkbare (5), enrd (5), dkobe (5), Nbun (5), *podip (3), enrip (3)

C

c common /Soze/ its, epss common /MaBasi/ R, H, ishape

C

c common /mabu' c5e, c8e,c9e,p9, cfpe, ftin

c U5, U8, PU9,FP concentrations at EOL (integrated fluence=ftin)

c all from subrou bupo.Per unit input volume

c

common /Bala/ Ereq, relr, relal, rela2,dkeol,dkstr, BU, Vcor, * U5m, dkbu, dkbe, rkre, dcr, sigma, pin, dpv, dkpv, tduco,

*eps, itest

c

C

c

c

common /cont/ Rad, dvp, drr, enr, pod, refec,ncr, dksu, dkest, relar, dcd

common/thermo/ tit, nnodo, ifri, npin, pint, tin, toutl, deltapa

*, Pow, rjs, flowt, podt, tduc, Rc

* Hact, Pfacr, Daxi, fact, iprth, iopth, ichoose

*, Pfacaz, iboun, nrod, nbu, nrodbu, hod

common / Conce/ coBe, coTa, coU5, cous, coli, coW, coRe, coHf, coHe, coN, coO common /Gamsh/

*dist, Req, shg ( 7), shgnb (7), Zeq (7), Dosewg (7), Doseg (7), itmag, epsg

*, aten, Dosen, Doseng (7)

c

c

Common /CROSS/ AU, BBU, signe, ishin, ishig

$\operatorname{Tsa}(\mathrm{ps})=8143 . /(8 .-\mathrm{a} \log 10(\mathrm{ps} / 0.133322))$

$\operatorname{Psa}\left(t_{g}\right)=0.133322 * 10 . * *(8.00-8143 . / t s)$

c

c

C
C

c

c

TLIm $=453.7$

read $(7,11)$ tit

11 format (a80)

Tsa(ps) is saturation temperature of $L i(i n K)$ as function of ps(KPa) Psa(ts) is " pressure of $\mathrm{Li}(\mathrm{in} \mathrm{KPa}) "$ " of $t s(\mathrm{~K})$

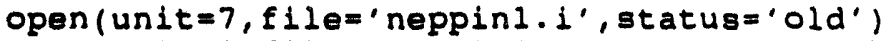
open (unit $=8$, file='neppinl. out', status='unknown')

c

Page B-1 


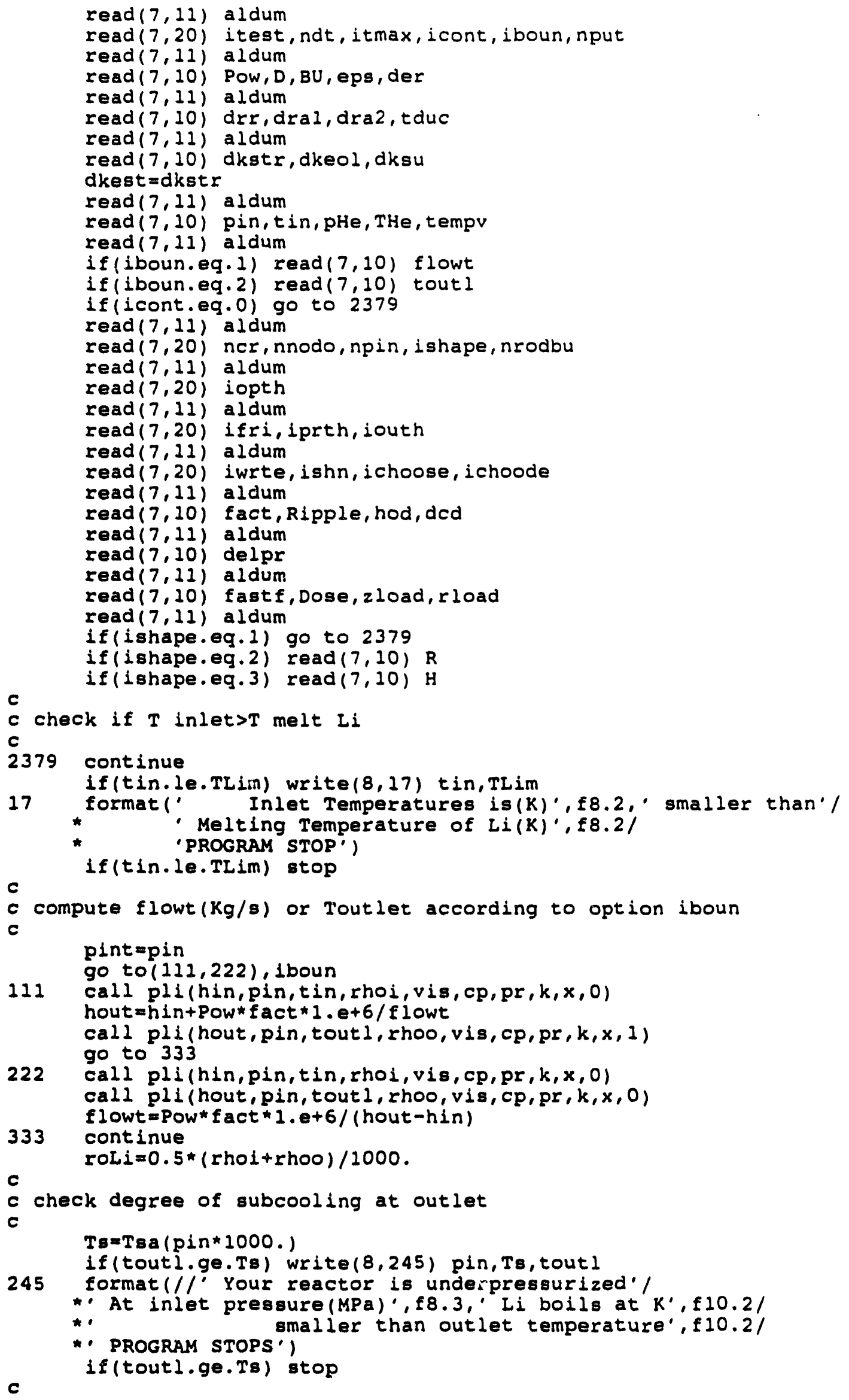


c

C

c

C

c

c

c

if (tempv.1t.0) tempv=tout 1

compute max stress (sigma) for pressure vessel

call desstr(tempv, 1,D,sigma, sigrod, itmax, ipresv)

epso=eps

epsi=eps

epsg $=$ eps

epss $=e$ ps

dero=der

$\operatorname{Dax} i=5$.

tduco=tduc

c

Ereq $=P O W * D * 365.25$

c

c

Core life in sec, multiply by 1 julian year $=31,557,600$, sec

tiempo $=\mathrm{D} * 3.15576 e+7$

c

c

c

C

c Pow (Mw): power; D(years): core life; BU(ats): percent U235 atoms

c burned;

c Inlet flow conditions:

c pin,tin(MPa,K)) pressure and temperature of the inlet coolant(fuel el

pHe, THe (MPa,K) initial" " " of He gap in rod.

drr, dral,dra2 (cm) thickness of Be reflector: radial, top-bottom axial

dkstr (8): reactivity structural materials(estimated)

dkeol(8): " at end of life(desired)

dkeu (8):desired subcritical reactivity required at BoL with drums in

eps: tolerance for iterations (like energy)

der $=1.01$ (compute derivatives)

fact: fraction fission energy/thermal

flowr ( $\mathrm{kg} / \mathrm{s})$ total coolant flow (applicable if iboun=1)

Caxi(cm): extrapolation length axial cos power distribution

c Ripple: Relative amplitude of power distribution as function of angle

c at $R=R$ core

c tduc(mm): thickness of the duct of the bundle of fuel elements

c hod: led of the spacing wire of the fuel rod/rod diameter

c dcd(cm): thickness B4C sheet of control drums

c tempv(K): Temperature of Pressure Vessel; if <0. program chooses tout

c delpr(pa): max delta preasure for core

c toutl(K): outlet temperature of coolant (applicable if iboun=2)

castf(1/cm2): fast fluence (during time D) at payload area

cload(m): axial distance from the base of reactor to payload plane

c rload (m): radius of load area

c Dose(Rad): integrated gamma dose(during time D) required for payload

$c$ ddt (cm): delta $r$ and delta $h$ for test curve Eavailable=f(R) or $f(H)$

c.

10 format $(7 e 10.0)$

c

$i \max =i t \max$
$i \max t=i t \max$
$i t \operatorname{mag}=i t \max$
$i t g=i t \max$

INPUT DESCRIPTION FOR INTEGERS

c

c

c itmax: max number iterations for reactor search

Page B-3 


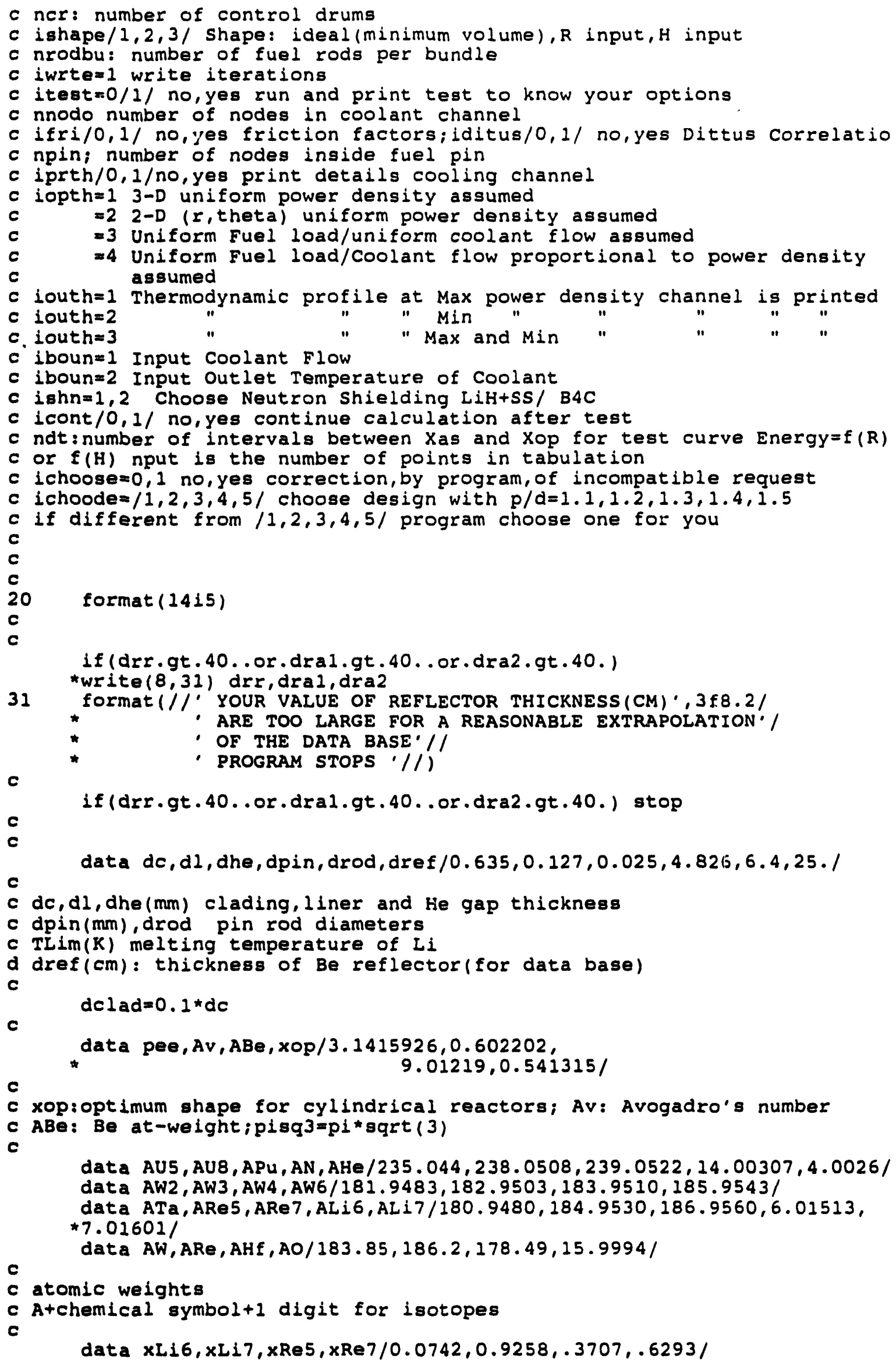




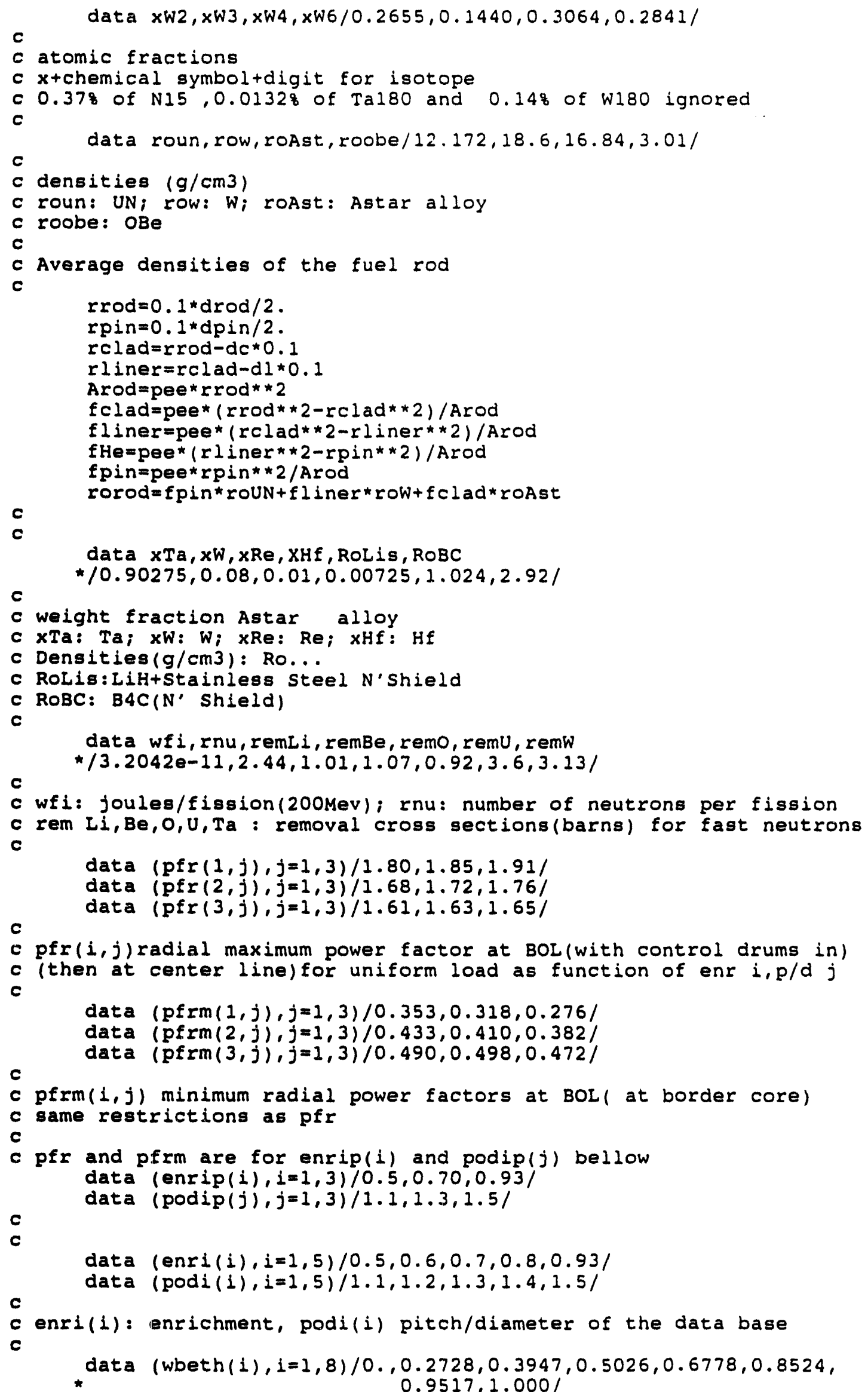




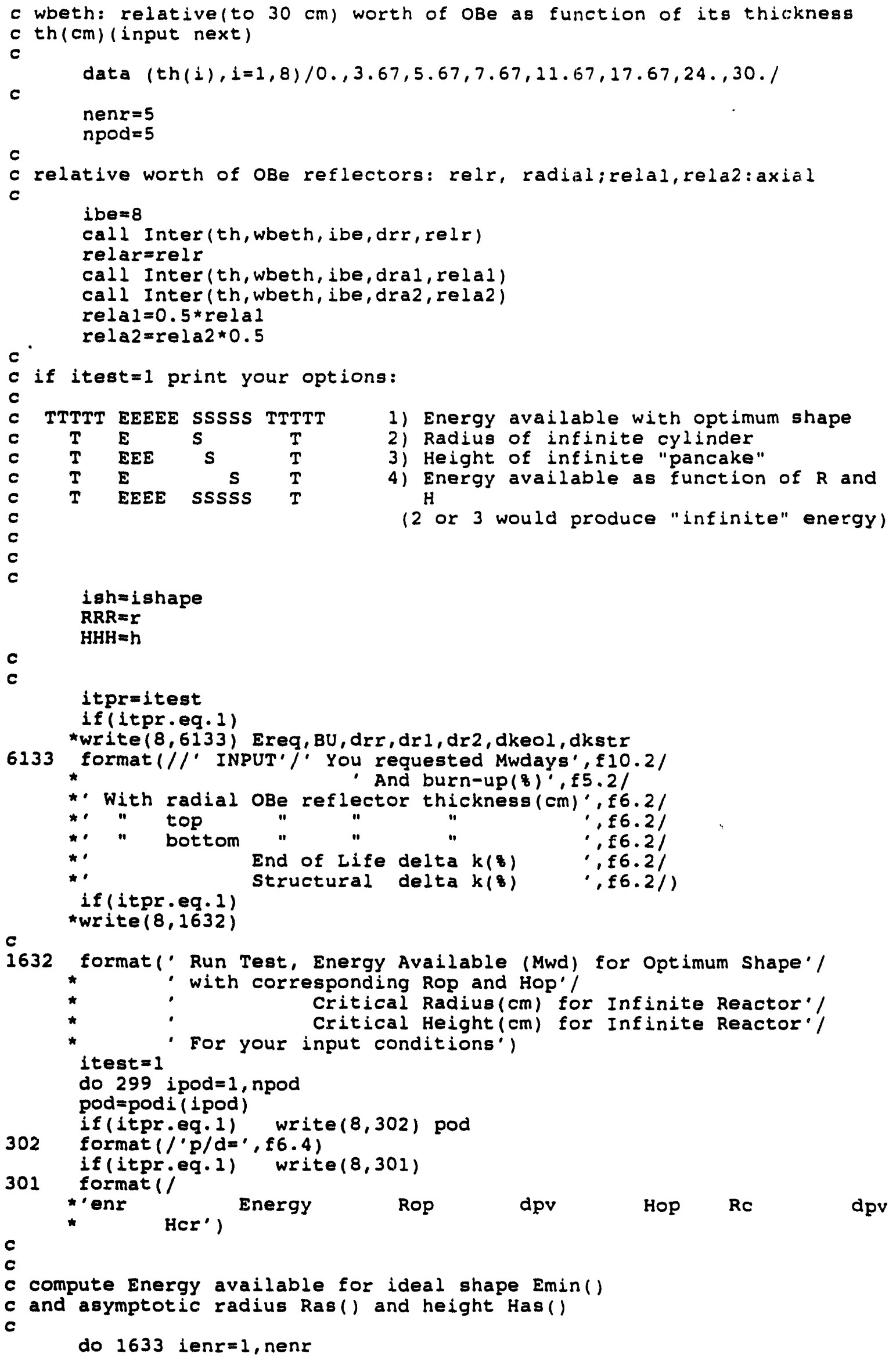

Page B-6 


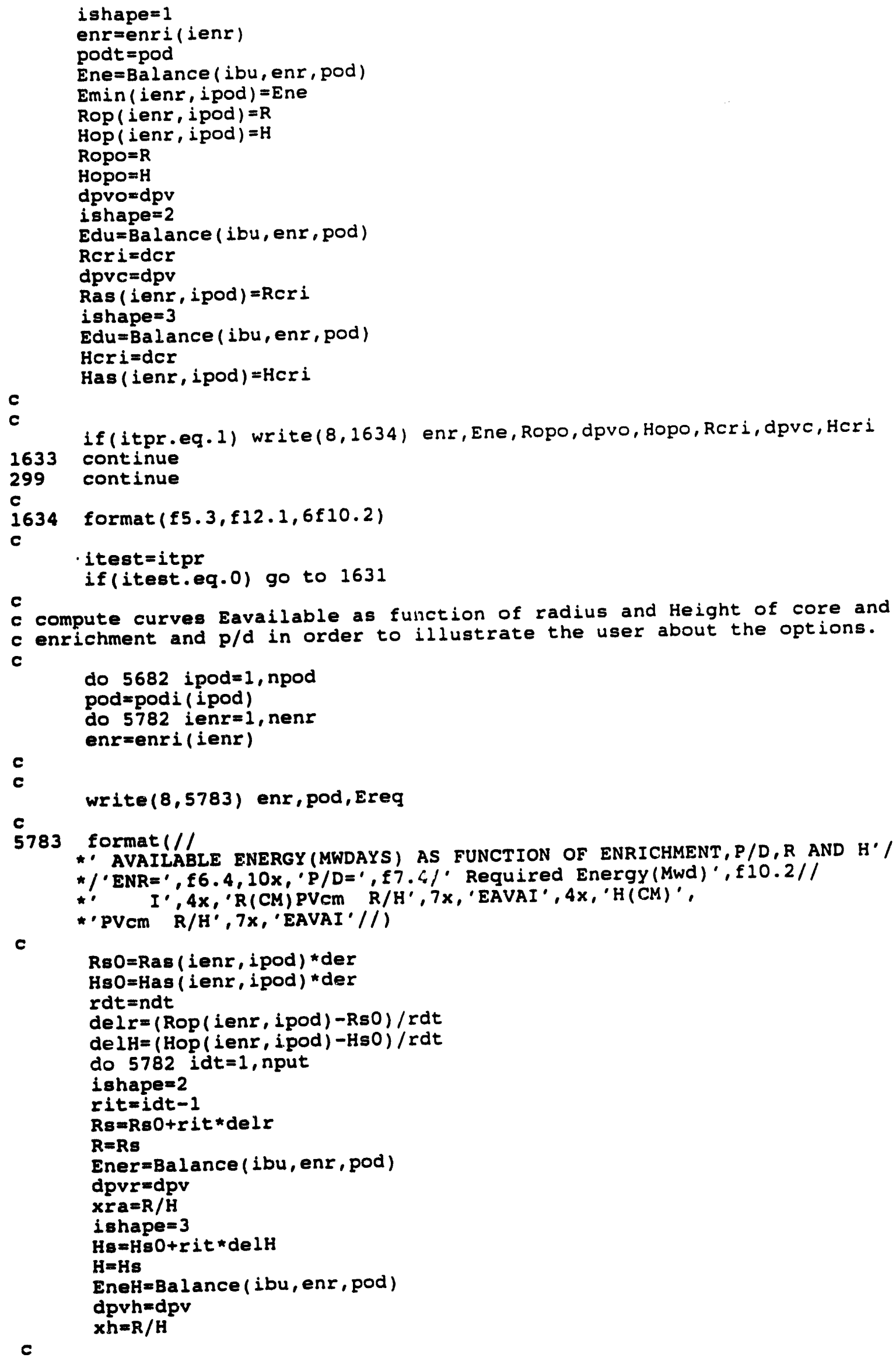


5784 format $(i 5,2(f 9.2, f 4.1, f 5.2, f 12.0))$

5782

5682

c

c

c

c

c

c

c

C

c

c

1631 continue

c stop here if your intentions are only to run the test

c

c

if(icont.eq.0) stop

icart $=1$

c

C

c

c

drodc $=0.1 *$ drod

do 467 ipod=1, npod

isha (ipod) $=i s h$

467 bual (ipod) $=$ bu

c

c

c

do 1391 ipod=1, npod

c start loop of designs by fixing first $p / d$

c up to statement \#1391

pod $=\operatorname{pod} i($ ipod $)$

BU=bual (ipod)

ishape $=i$ sha (ipod)

C Abu and Abund(cm2) area of 19 rods bundle with and without the duct Abu $=3.4641016 *$ drodc $* 2 *($ pod $* 2.73205081-0.5+$ tduc $/$ drod $) * * 2$ Abund $=3.4641016 \star d r o d c * \star 2 \star(\operatorname{pod} \star 2.73205081-0.5) \star \star 2$

c duct fraction

fduct $=($ Abu-Abund $) / A b u$

c $11,2,3$ are flow fractions for cell types $1,2,3$ (with respect to acell1) $x=$ pod

$f 1=1 .-0.906900 *(1 .+(x-1) * * 2 / 3.) / x * * 2$

$f 2=2.30940 *((x-.5) * x-.392699 *(1 .+.5 *(x-1). * \star 2)) / x * \star 2$

$f 3=2.30940 *(.577350 *(x-.5) * * 2-0.130900) / x * \star 2$

c area of cell (cm2) is acell

pitch $=$ pod $*$ drodc

acell $=0.433013 *$ pitch $* 2$

c a1,a2, a $3(\mathrm{~cm} 2)$ are area flow of cell types $1,2,3$

al $=f 1 * a c e l l$

$a 2=f 2 * \operatorname{acel} l$

$a 3=f 3 *$ ace 11

At $=24 . \star a 1+12$.*a2+6.*a3

c flow fraction

fLi=At / Abu

Arod $=$ pee*rrod**2*19.

c rods fraction

frod $=$ Arod $/$ Abu

c spacing wire fraction 


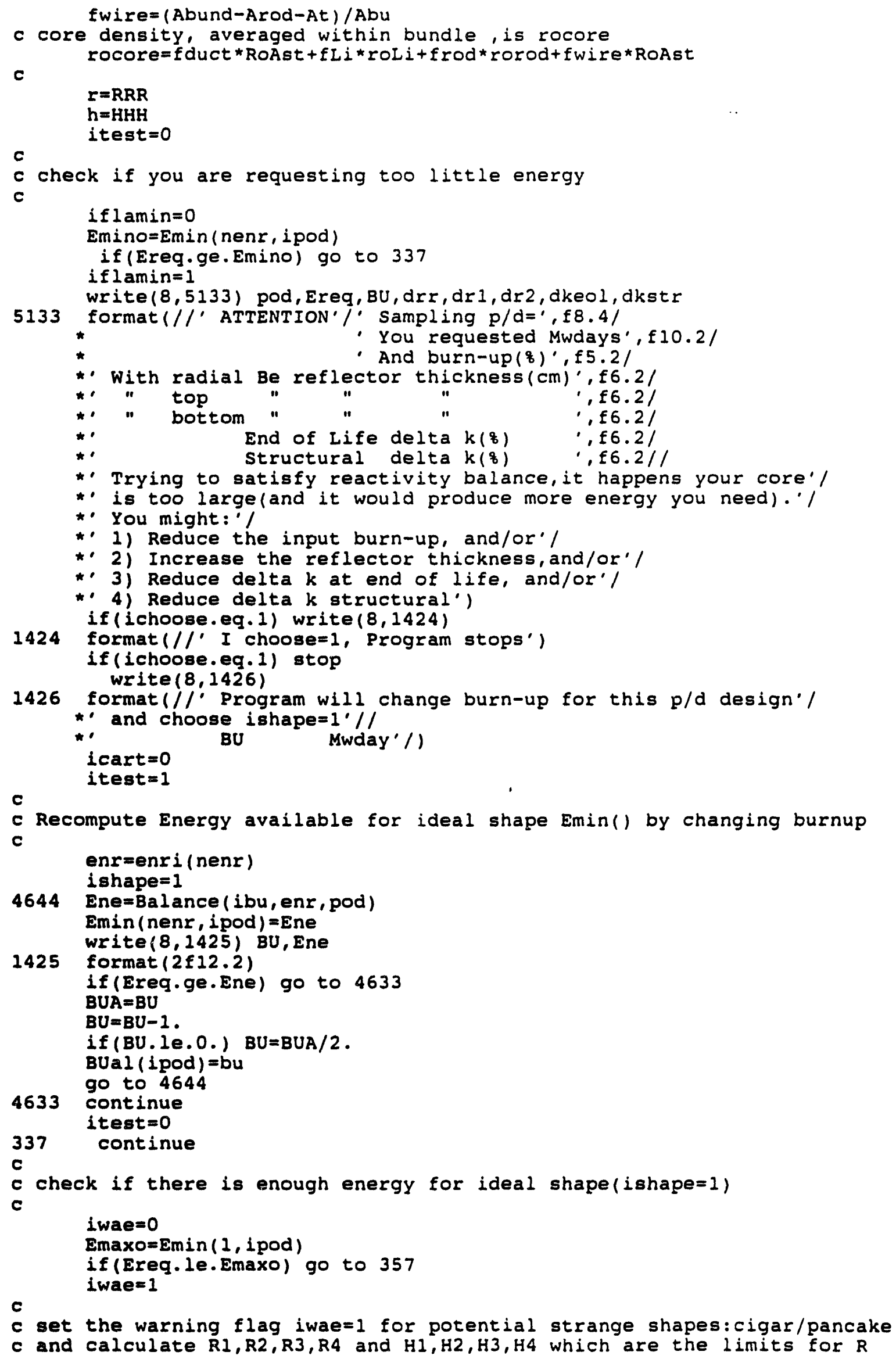


$c$ and $H$ for the input conditions(reactivity, required Energy) in the c bifurcation region

c

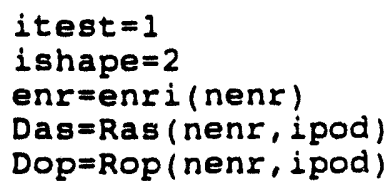




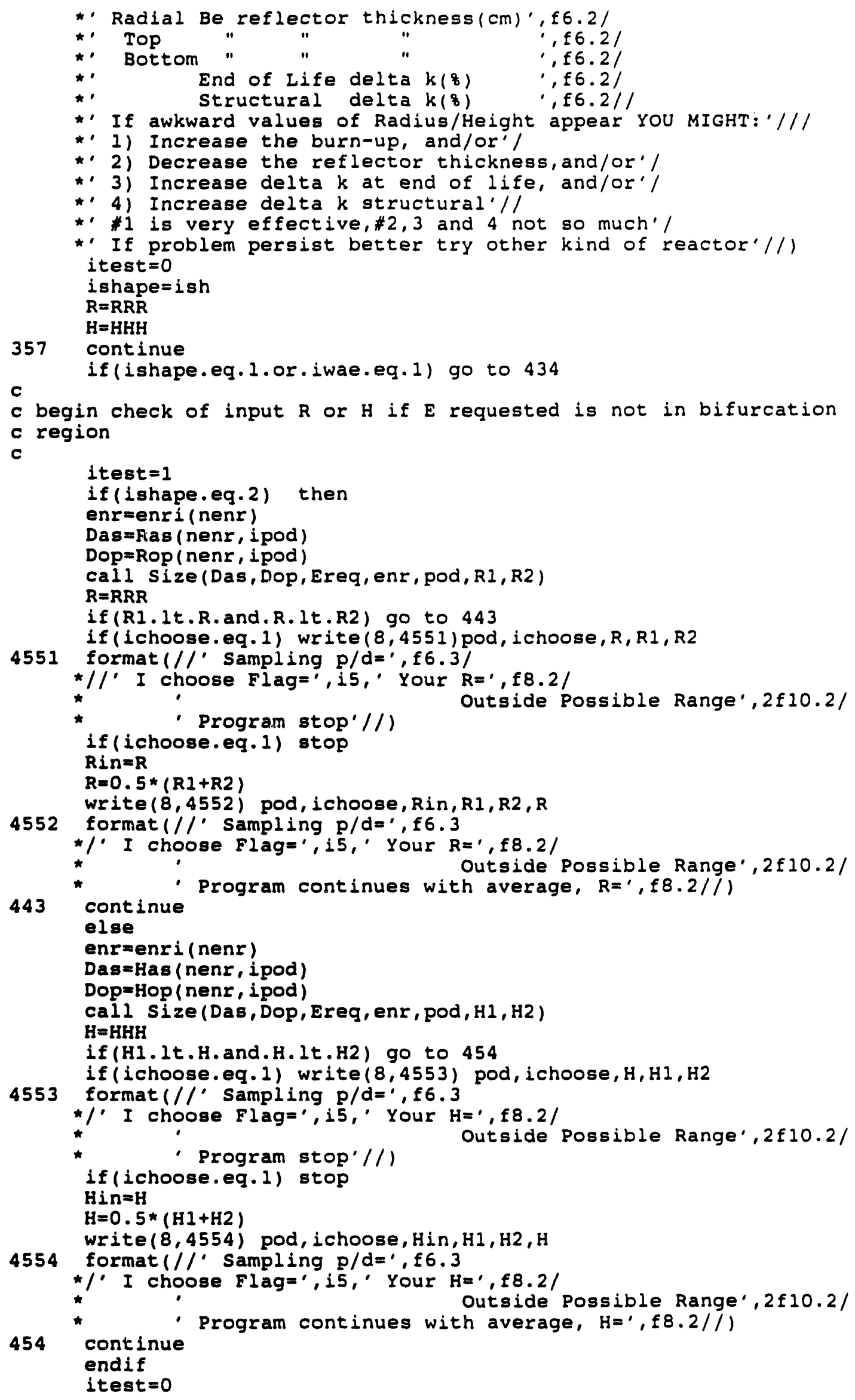




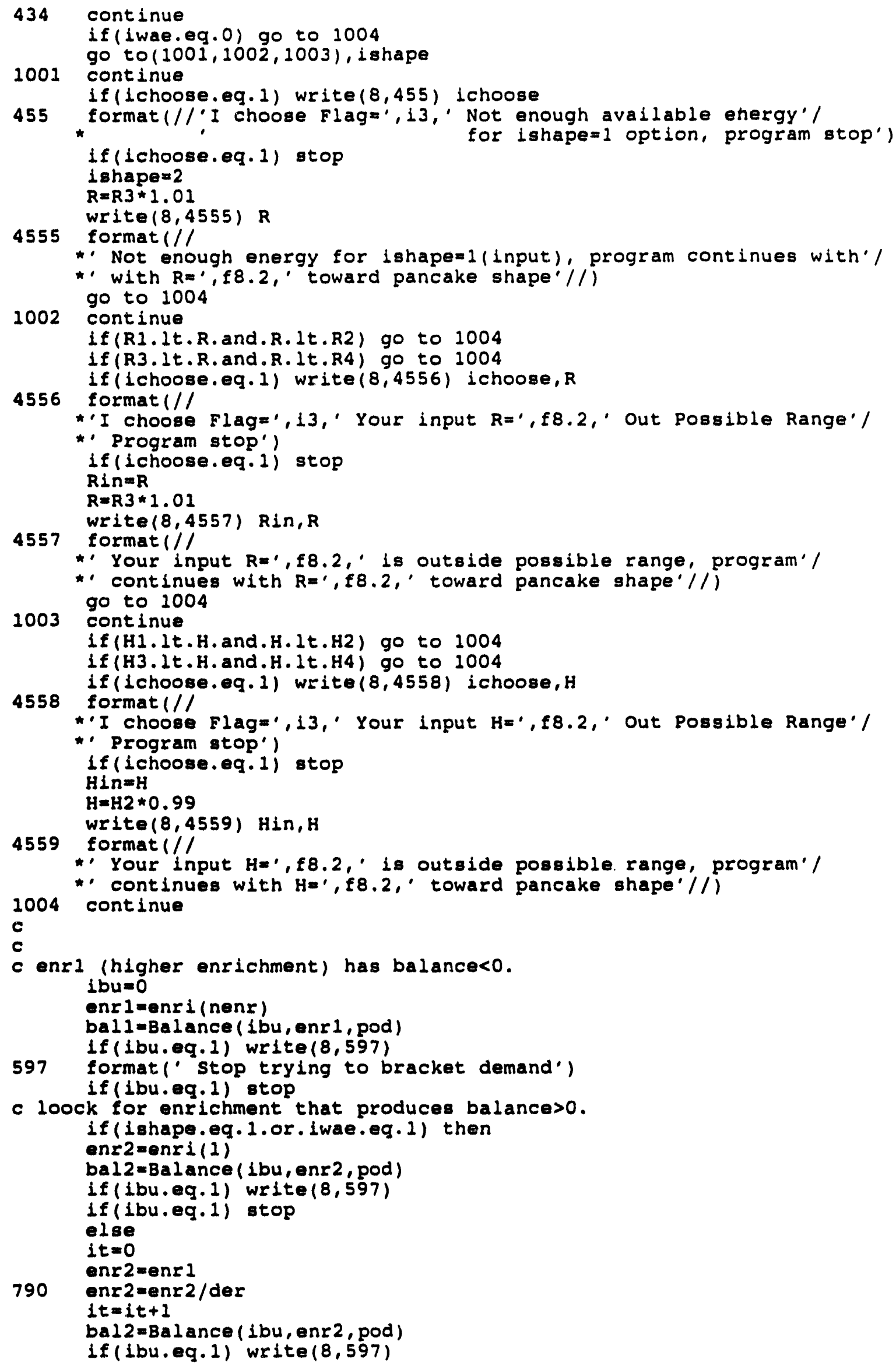




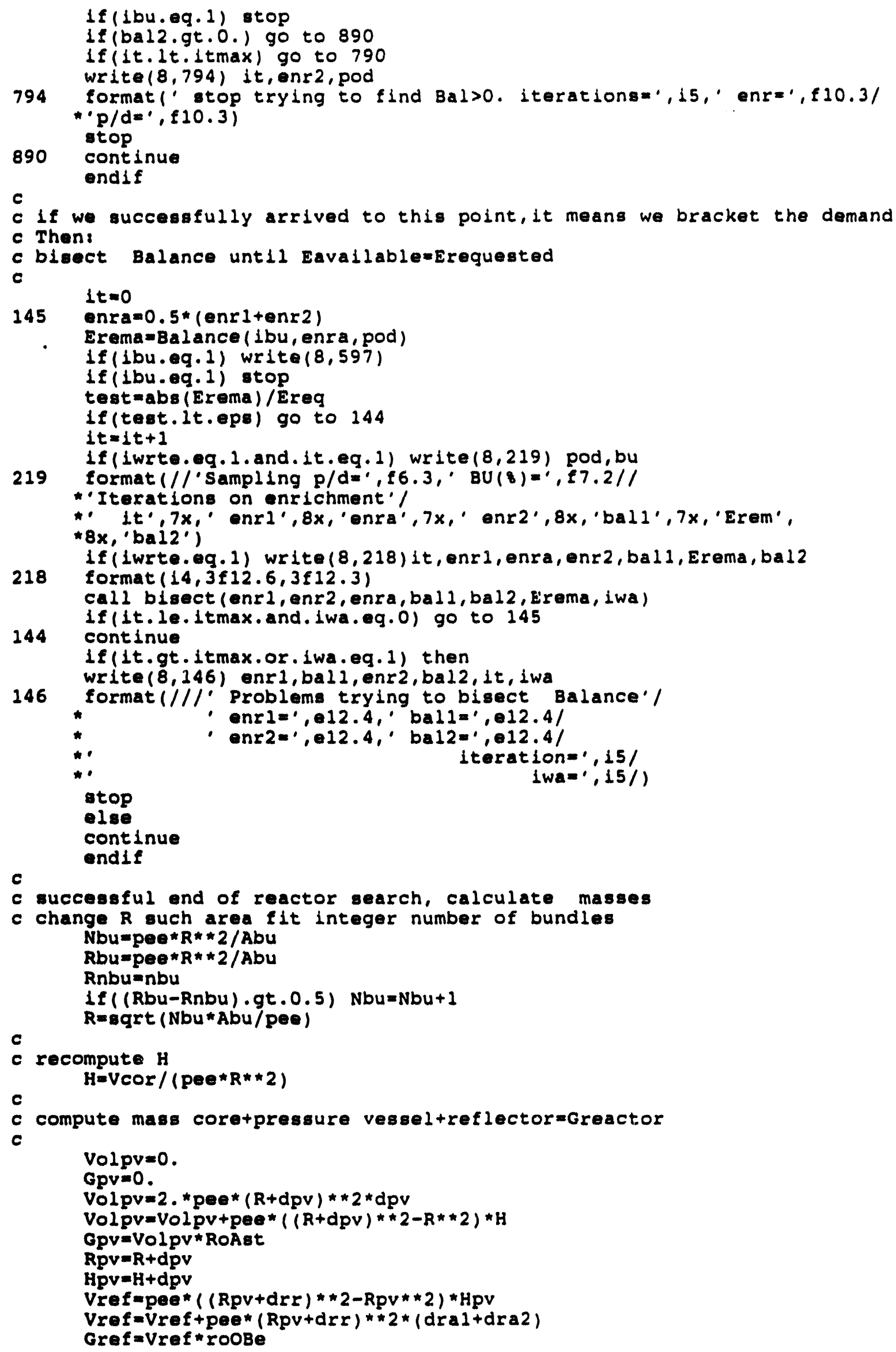




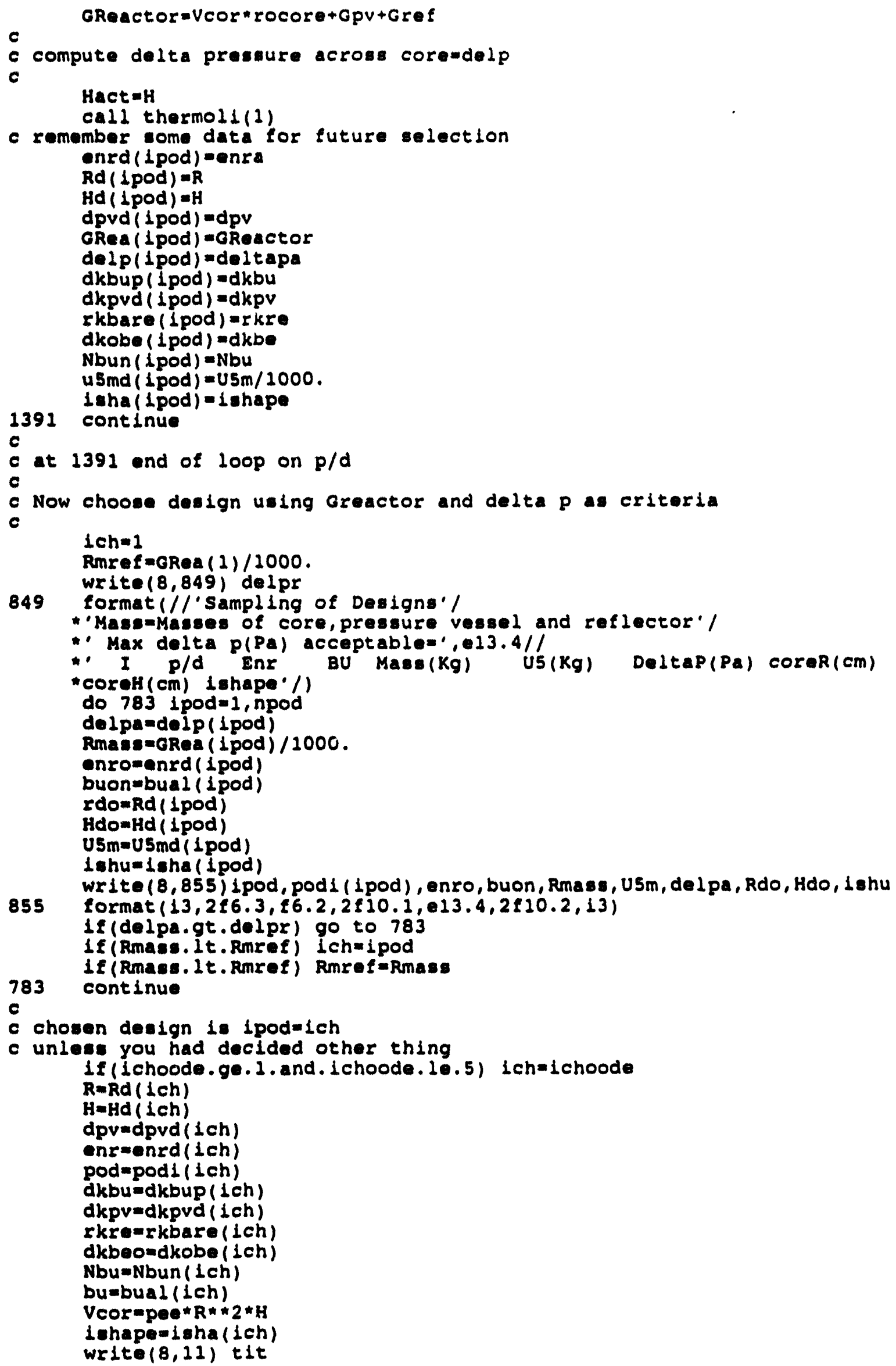

ICh=1

Rmref =GRea ( 1 ) / 1000 .

write $(8,849)$ delpr

849 format (//'sampling of Designs'/

- 'Masemasees of core,pressure vessel and reflector'/

- Max delta $p(P a)$ acceptablea', e13.4//

*I P/d Enr BU Mass(Kg) U5(Kg) DeltaP(Pa) coreR(cm)

* coreh (cm) Ishape $/ 1$

do 783 ipod=1, npod

delpandelp ( Ipod)

Rmass=GRea ( I pod) / 1000 .

enro=enrd (lpod)

buon=bual ( 1 pod)

rdomRd (ipod)

HdomHd (1pod)

USm=U5md ( I pod)

Ishu= I ha (ipod)

Write $(8,855$ ) ipod, podi ( L pod), enro, buon, Rmass, U5m, del pa, Rdo, Hdo, Ishu

855 format $(13,2 \notin 6.3,26.2,2 f 10.1,13.4,2 \notin 10.2,13)$

if (delpa.gt.delpr) go to 783

if (Rmass. It.Rmref) ich=ipod

if (Rmase. 1t.Rmref) Rmref=Rmass

783

continue

c

c chosen design is ipod=ich

c unleas you had declded other thing

if (1choode.ge.1. and.ichoode.1e.5) ichmichoode

$R=R d$ ( $1 \mathrm{ch}$ )

$H=H d$ ( $1 \mathrm{ch})$

dpvadpvd (1ch)

enr=enrd (Lch)

podmpodi (1 ch)

dkbu=dkbup (1ch)

dkpvedkpvd ( $1 \mathrm{ch}$ )

rkre=rkbare (ich)

dkbeondkobe (1ch)

Nbu=Nbun (1ch)

bu=bual (lch)

Vcor=pee*R**2*H

ichape=isha (ich)

write $(8,11)$ tit

Page B-14 
write $(8,51)$ Pow, D, Ereg, Bu,drr,dral, dra2, eps

c 1

format $/ / /^{\prime}$ INPUT TO THE PROGRAM $/ / /^{\prime}$ Power $(M w)^{\prime}, f 8.2,^{\prime}$ Core 1 ife' $t$

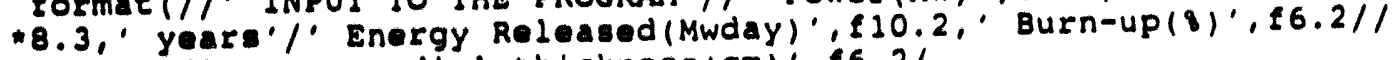

* Be reflector, radial thickness $(\mathrm{cm}),, 26.2 /$

$\cdot 0$

top

16.21

* bottom " $16.2 / 1$

- The following design can produce that energy within the relative

- / numerical precision of',ed2.41

c

If (1 ichape.eq.1) write $(8,317)$ xop

317

format (/" Minimum Core Volume Option Chosen: $R / H=1, f 9.6 /)$

c

1f (1ahape.og.2) write $(8,318)$ R

318 format (/" Core Radius Option Chosen: $R(\mathrm{~cm})=\cdot, f 7.2 /)$

C.

Lf (ishape.eq.3) write $(8,319) \mathrm{H}$

319 format (/, Core Height Option Chosen: $H(\mathrm{~cm})=1, f 7.2 /)$

c

dkfp=dkbu

$d k r e q=d k f p+d k=t+d k e o l+d k p v$

dkbeo=dkbeo/ 100 .

refecerkre+dkbeo

c

c write $(8,52)$ dkreg, dkfp, dkpv, dkstr, dkeol, refec, rkre, dkbeo

c

52

format (' And it has the following reactivity balance'/

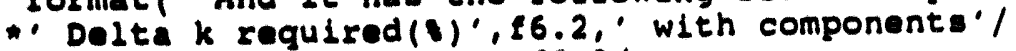

- Fiesion Products ( 1$)^{\prime}, 86.21$

* Presenre Veseed (\$), 16.21

- Structural (\$), .66 .21$

* End of Life(s)'.26.2/

" keff at BoL(No etructural mat)',f7.4, ' Components'/

* Bare Reactor', 17.4 /

* Reflector. $\$ 7.4 / 1$

8

6

write $(8,521)$ ens,pod

521 Lormat(' Enrichmont=', 18.4, P1tch/Diameter=',f6.2)

e

Rad $=\mathbf{R}$

c

call control to design control drums

c

dvpadpv

Call Control

c

c

nrod=Nbu*nrodbu

Veoreveor $/ 1000$.

c

densities inside the pin

AU=enx*AUS + (1.-OnX) *AUB

$A U N=A U+A N$

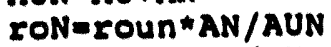

rOU = roun"AU/AUN

rou $5=$ anr $* A U 5 *$ rou $/ A U$

rou $8=(1 .-0 n r) * A U 8 * \mathrm{rOU} / \mathrm{AU}$

CU5 $=$ rOU 5 *AV / AU5

CU8 $=$ rOU $8 * A v / A U 8$

Vact $=$ nrod*pee* (drodc/2.)**2*H*Epin/1000.

usm=Vact* rous

$48 \mathrm{~m}=\mathrm{Vact}$ * rou 8

Pum $=0$.

Epm=0.

Page B-15 


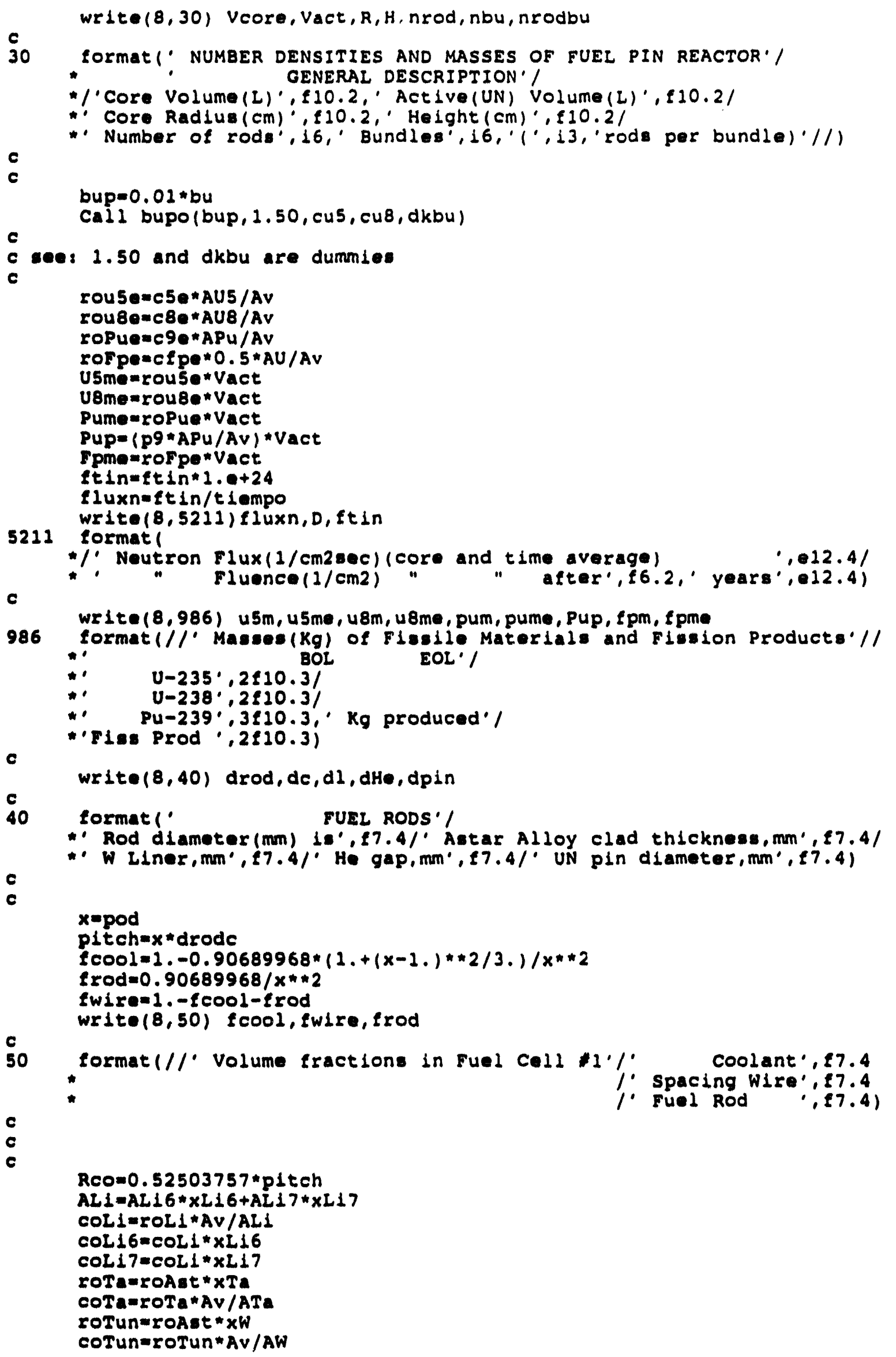

Page B.16 


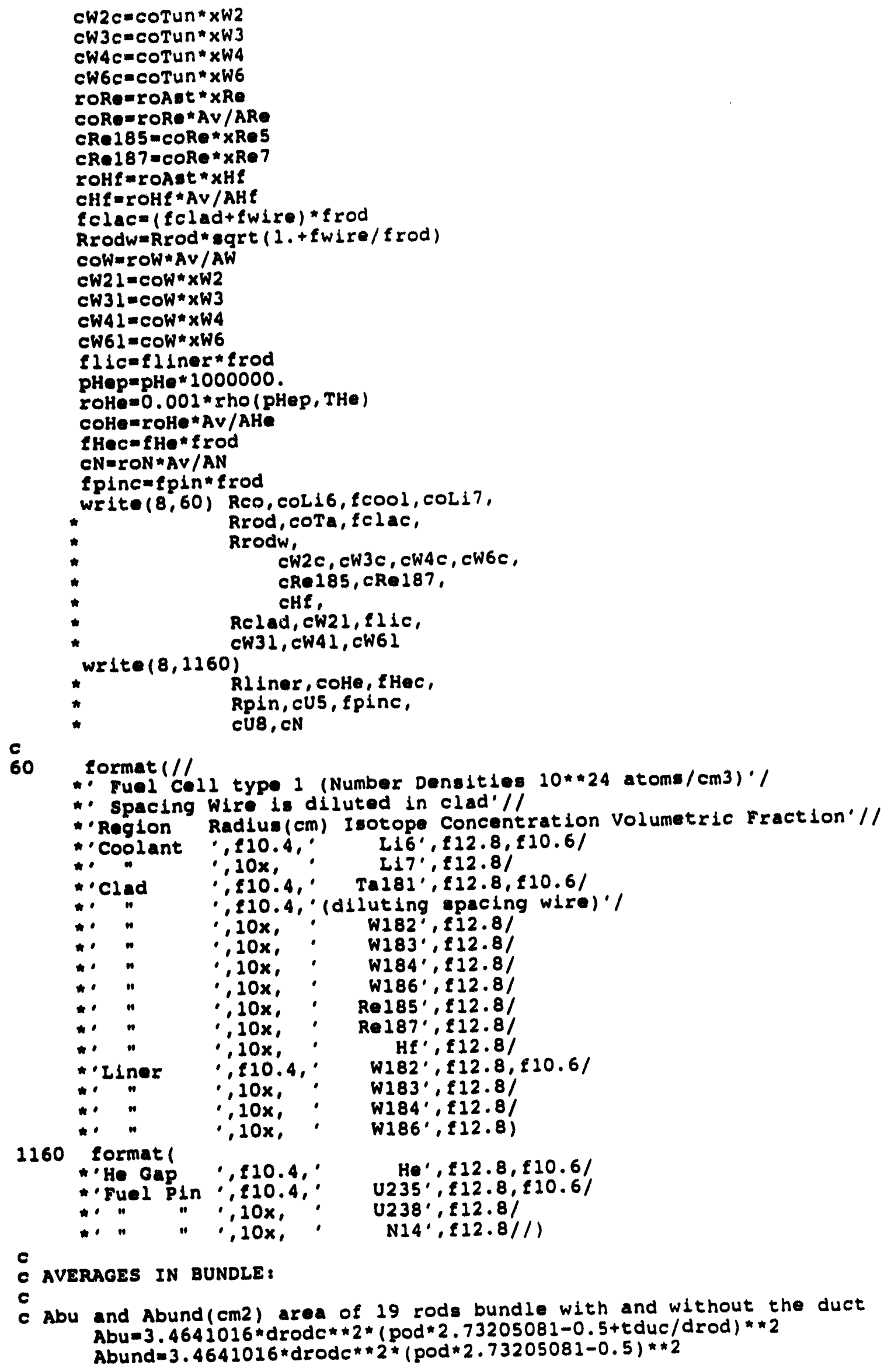

Page B-17 


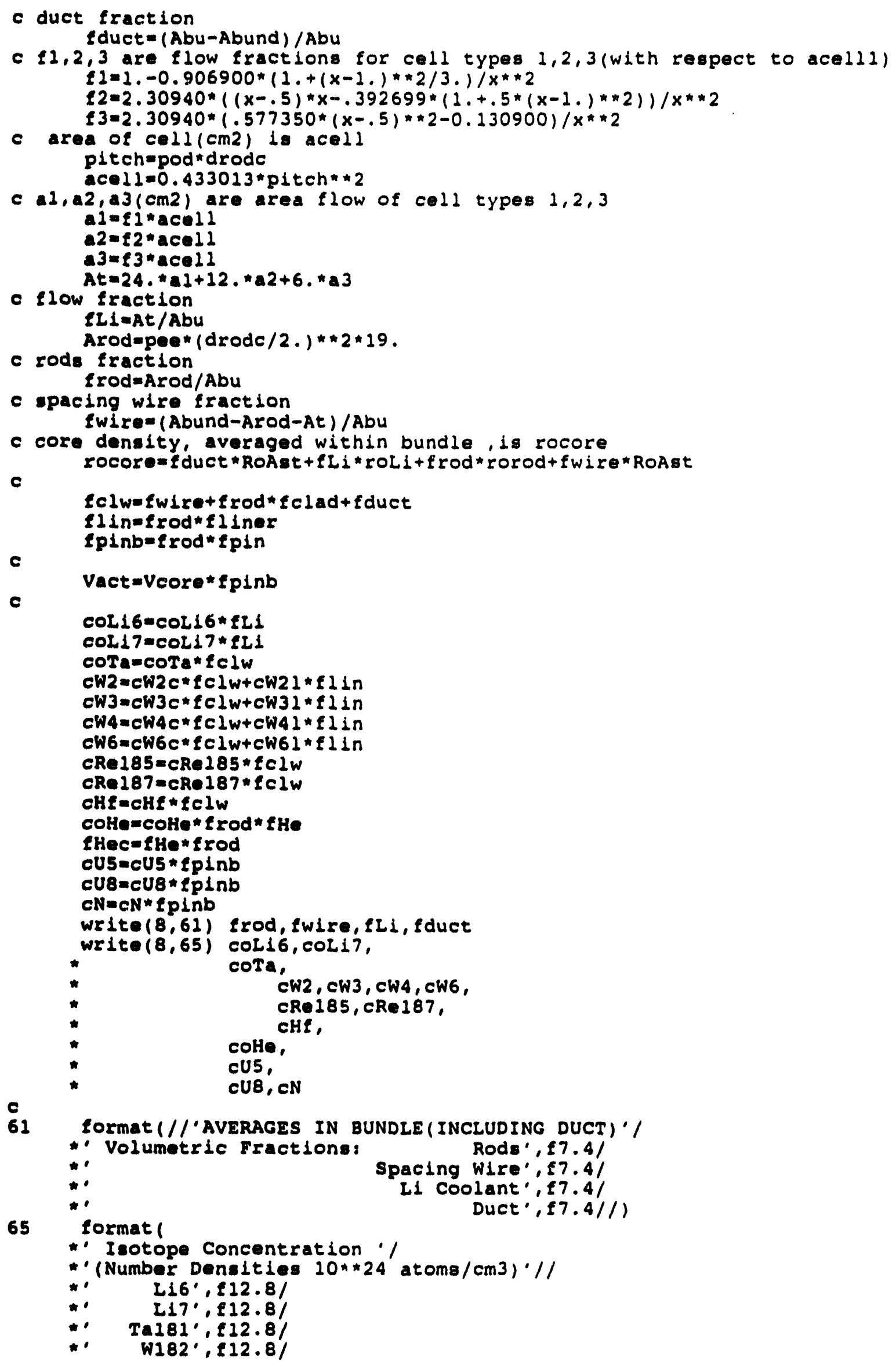




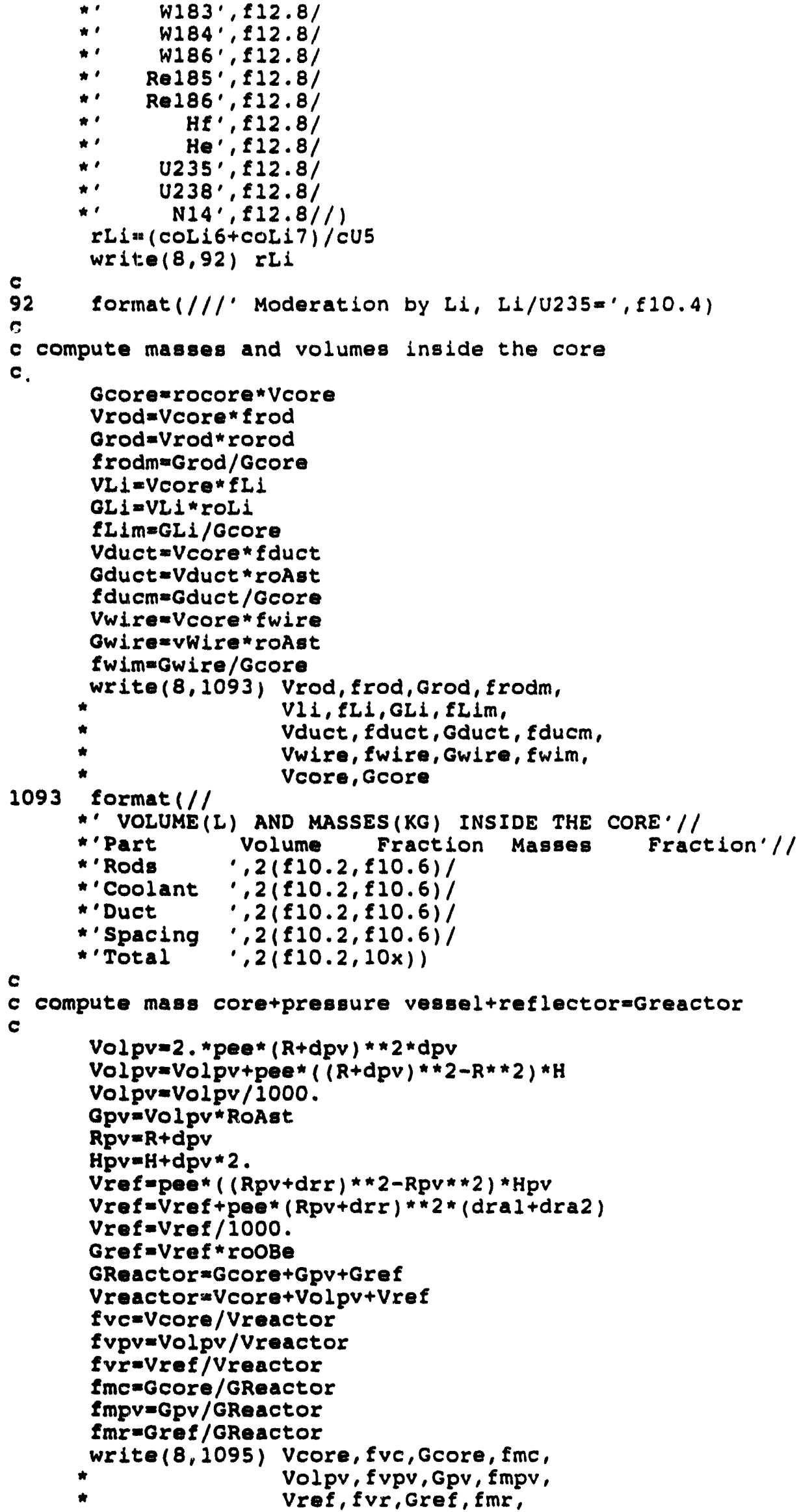




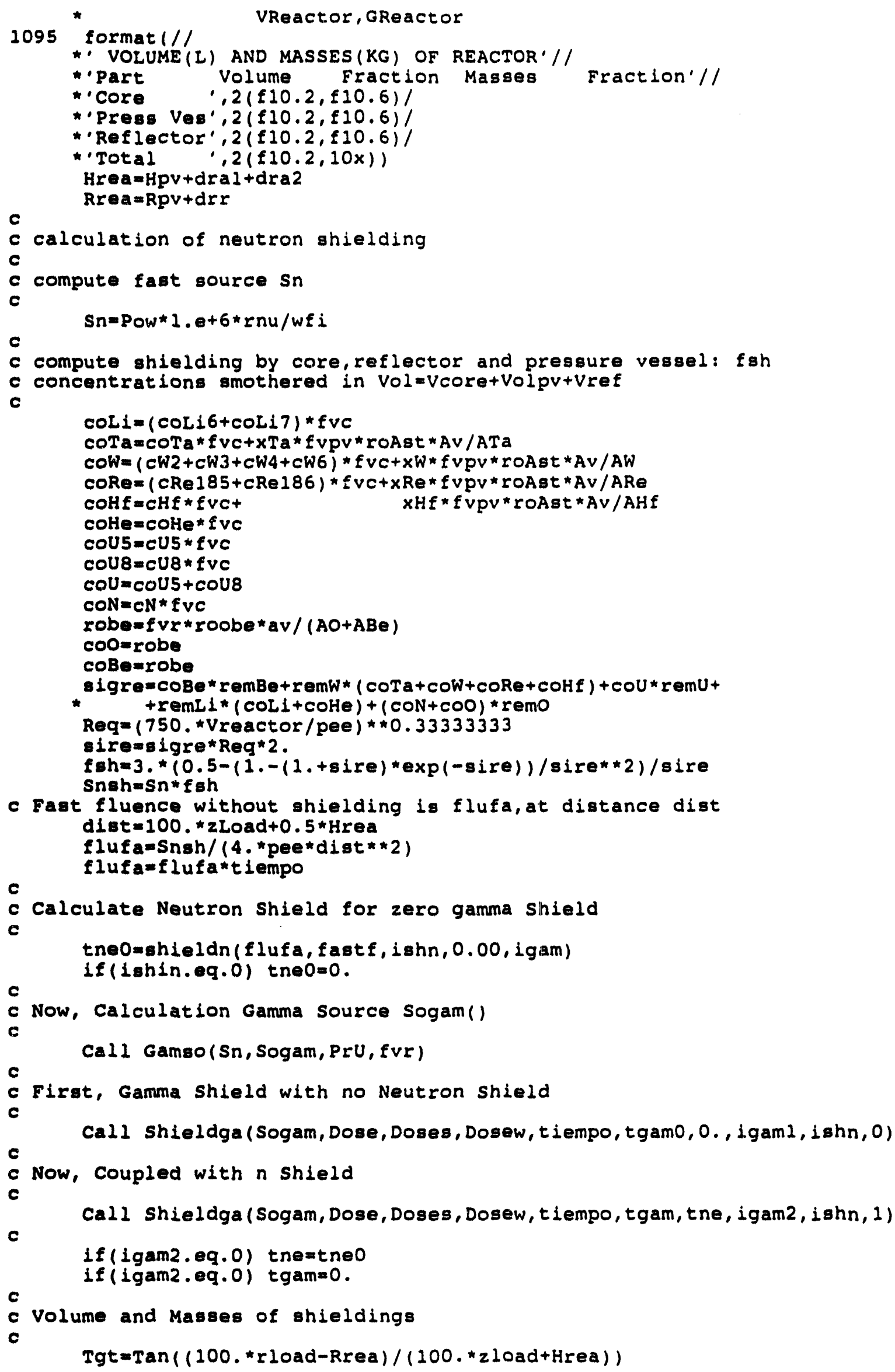




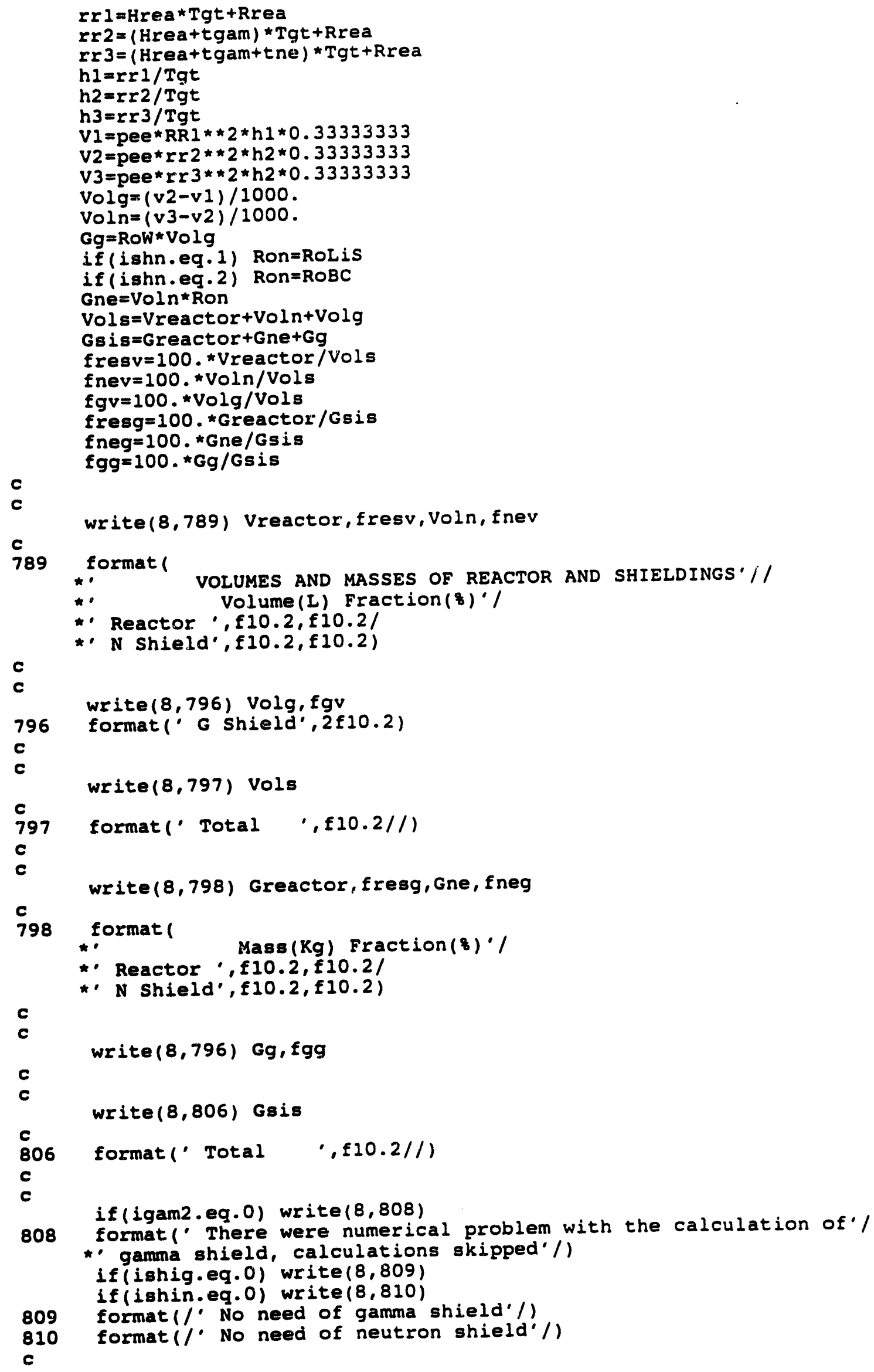




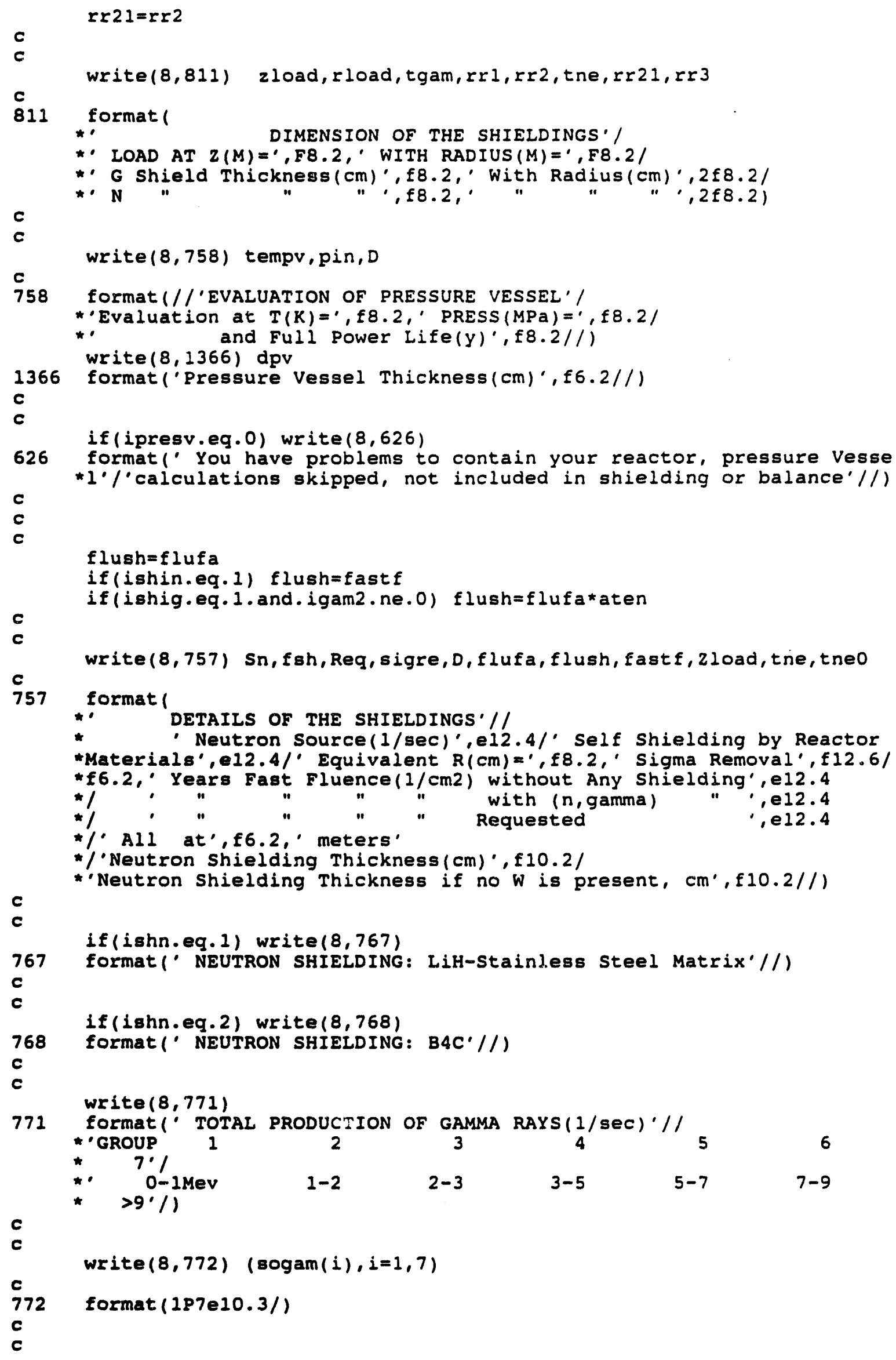




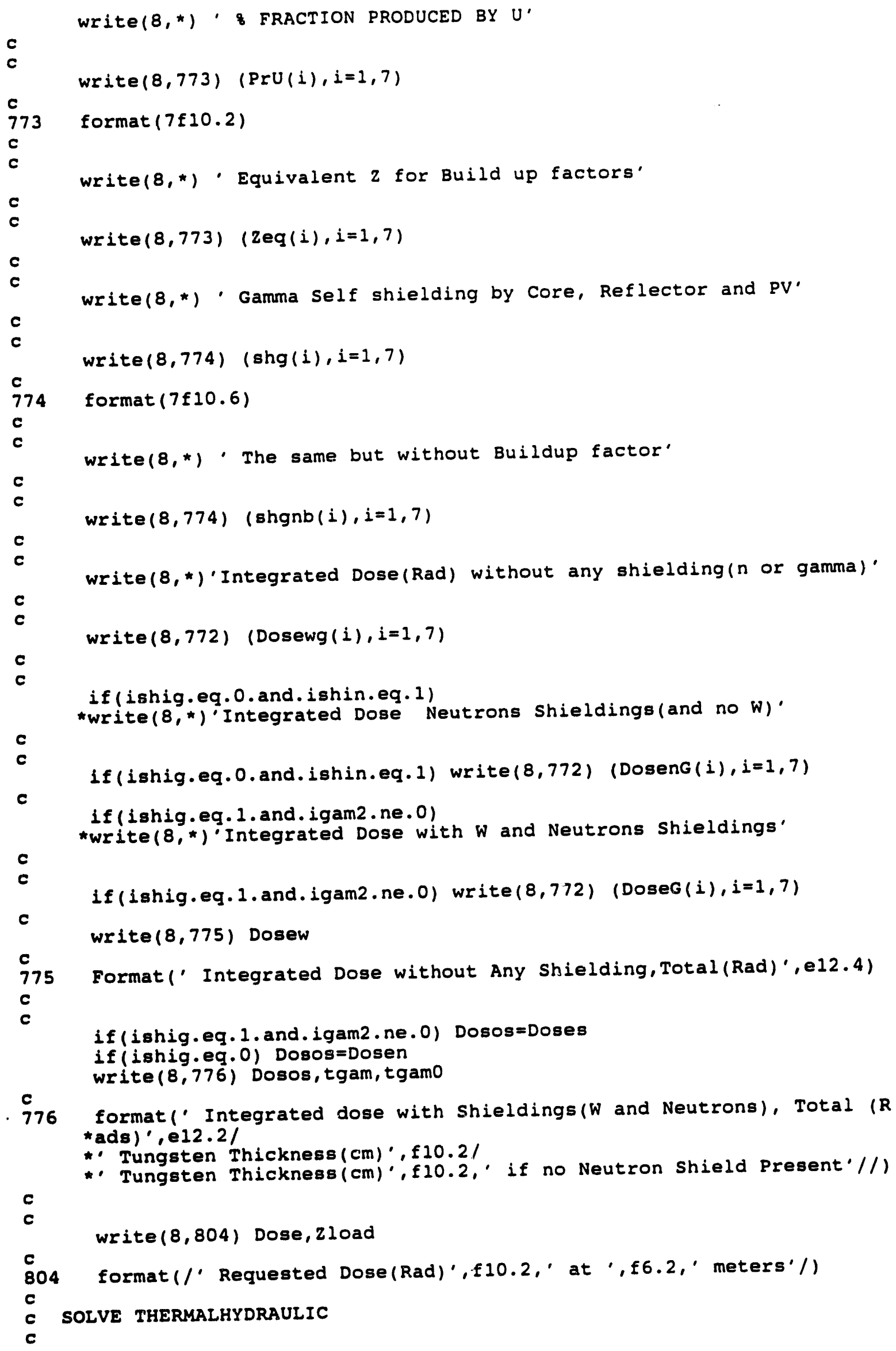




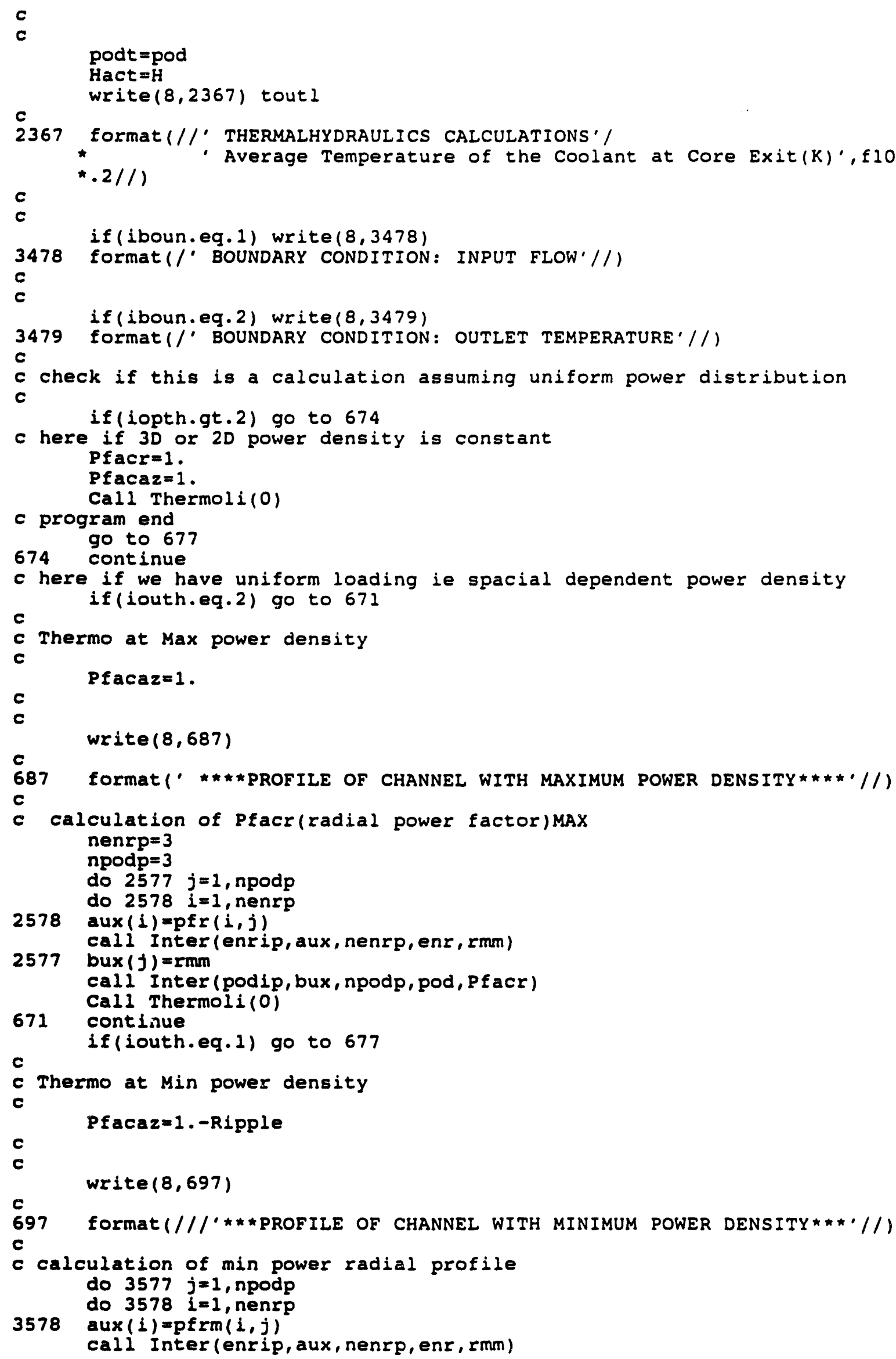


3577 bux $(j)=r m m$

call Inter (podip, bux, npodp, pod, pfacr)

call Thermoli(0)

677

continue

Stop

c

End

c

c

c

c

c He density $(\mathrm{kg} / \mathrm{m} 3)$ function of $\mathrm{p}(\mathrm{Pa})$ and $t(\mathrm{~K})$

c from page 405, ANS book "Thermal and Flow Design of Helium-Cooled

c Reactors",G. Melese and R. Katz

c

Function rho $(p, t)$

$R=2077.22$

$r h o=p /(r * t+p * b(t))$

return

end

C

c Function $b(t)$ (used by rho)

Function $b(t)$

data $c 1, c 2, c 3, c 4, c 5 / 9.489433 e-4,9.528079 e-4,3.420680 e-2$,

$\star$

$2.739470 e-3,9.409120 e-4 /$

$b=c 1+c 2 /(1 .-c 3 * t)+c 4 /(1 .+c 5 * t)$

return

end

Compute available energy or

difference between available and requested energies

as function of $S / F$ (other thing too)

Function Balance (ibu, enr, pod)

For input enr(ichment), pod(p/d) and when itest $=0$ :

this function calculates 1) the volume of the core that satisfies the

reactivity balance; and then 2 ) the energy balance (Mwday)

Balance=Eavailable-Erequested. If ishape $=2$ or 3 (input $R$ or $H$ ) the

function checks if $R(O r) H$ is compatible with reactivity balance

if not, set flag ibu=1 and RETURN

if itest $=1$ and for inputs enr and pod it calculates

available energy (for ishape $=1,2$ or 3 ),

radius infinite core that satisfies reactivity balance if ishape=2

height of infinite " " " " " " "

c

common /Bala/ Ereq, relr, relal, rela2,dkeol,dkstr,BU,Vc,

* U5m, dkbu, dkbe, rkrem, dcr, sigma, pin, dpv, dkpv, tduc,

*eps, itest

c

common /MaBasi/ R, H, ishape

c

dimension $\operatorname{rm}(5,5), \operatorname{enr} j(5), \operatorname{pod} i(5)$, bew $(5,5)$

$\star, r k i(5,5), \operatorname{prv}(5,5), \operatorname{aux}(5), \operatorname{bux}(5), \operatorname{dext}(5)$

C

data $(\mathrm{rm}(5, j), j=1,5) / 7.695,8.762,9.841,10.927,12.010 /$

data $(\mathrm{rm}(4, j), j=1,5) / 7.945,9.032,10.131,11.230,12.325 /$

data $(\operatorname{rm}(3, j), j=1,5) / 8.149,9.248,10.357,11.470,12.571)$

data $(\operatorname{rm}(2, j), j=1,5) / 8.356,9.467,10.586,11.706,12.811 /$

data $(r m(1, j), j=1,5) / 8.563,9.681,10.805,11.929,13.037 /$

e

Page B-25 


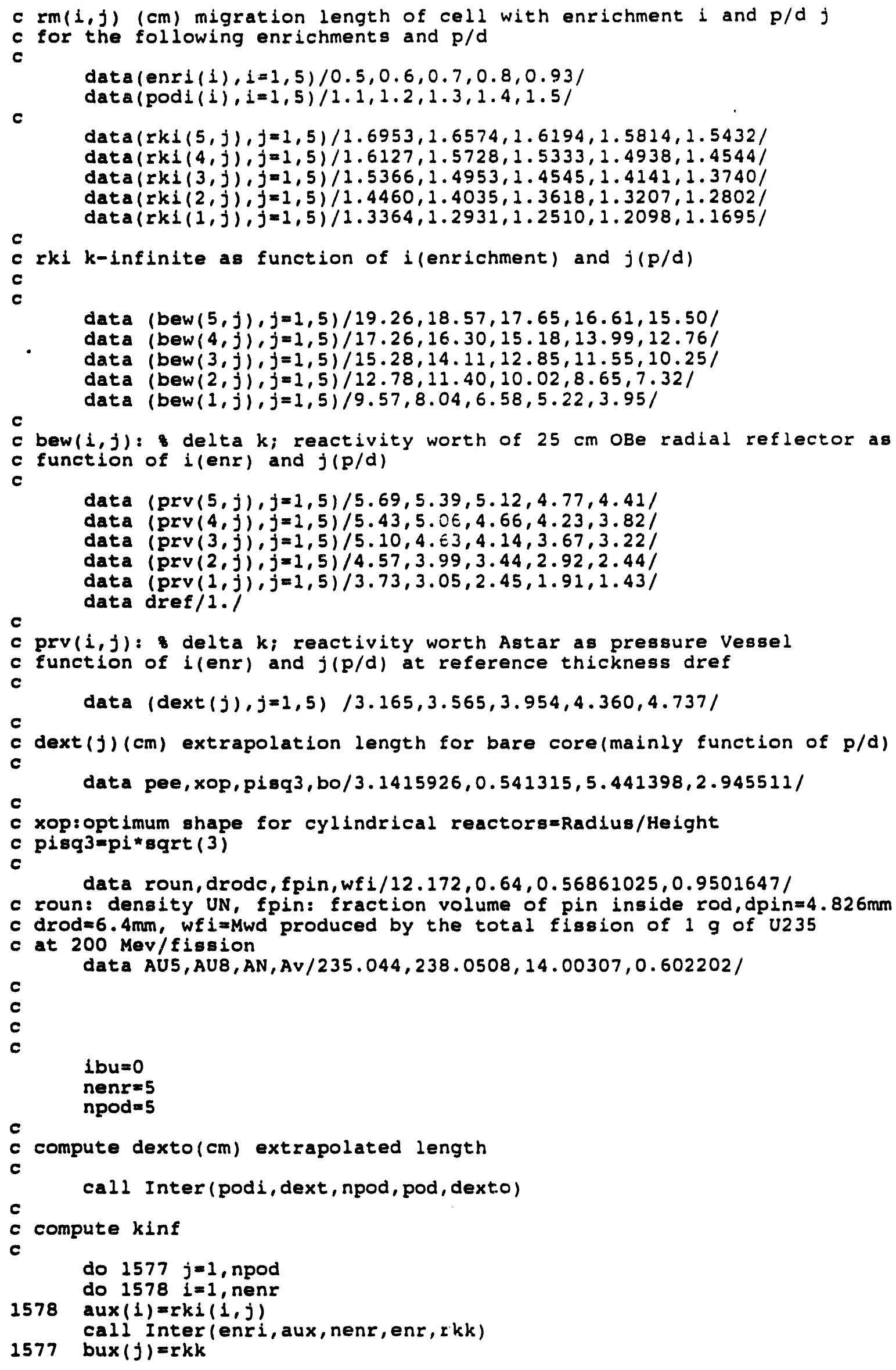


call Inter (podi, bux, npod,pod, rkin)

c

c compute migration length

c

do $2577 \mathrm{f}=1$, npod

do $25781=1$, nenr

$2578 \quad \operatorname{aux}(1)=\mathrm{rm}(1, j)$

call Inter (enri, aux, nenr, enr, rmm)

2577 bux $(j)=r m m$

c

cal1 Inter (pod1, bux, npod, pod, rmi)

c compute dk OBe reflector

c

do $3577 f=1$, npod

do $3578 i=1$, nenr

3578 aux $(1)=\operatorname{bew}(1, j)$

call Inter (enri, aux, nenr, enr, bmm)

3577 bux (j) $=\mathrm{bmm}$

call Inter (podi, bux, npod, pod, wbe)

wber =wbe*relr

wbea 1 =wbe*relal

wbea2 = wbe* rela2

dkbe=wber +wbeal + wbea 2

c

cb compute dk Astar pressure vessel (1. cm thick, reference value)

c

do $4577 f=1$, npod

do $4578 i=1$, nenr

4578 aux $(1)=\operatorname{prv}(1, j)$

call Inter (enri, aux, nenr, enr, bmm)

4577 bux $(j)=b m m$

call Inter (podi, bux, npod, pod, wpv)

Rref =bo*rmi /sqrt (rkin-1.)-dexto

drefp=Rref* (pin/sigma)

$a p v=r e l r * 0.01 * w p v *(0.4 / d r e f) * R r e f$

c

$d k p v=(d r e f p / d r e f) * r e l r * w p v$

c compute delta $k$ BU

c see: c50 and $c 80$ are number densities inside the pin

c

$A U=e n r * A U S+(1 .-e n r) * A U 8$

$A U N=A U+A N$

$C U=$ roun*Av/AUN

c50=cu*enr

c80 $=c u *(1 .-e n r)$

bup $=\mathrm{BU} * 0.01$

CaII bupo (bup, rkin, c50,c80, dkbu)

c

c compure $\mathrm{U} 235$ density diluted in the core, rous

c

rkrm=1 + $+(D K B U+d k s t r+d k e 01-d k b e) / 100$.

rkrem $=r k r m+d k p v / 100$.

c

go to $(2001,2002,2003)$, Lehape

c if Ishape=1, opt imum shape--- $>2001$

$2001 R p=r m i * b o / s q r t(r k i n / r k r e m-1$.

$H p=R p / x O p$

$R=r p-$ dexto

$d p v=(p i n /$ sigma ) *R

$H=h p-d e x t o * 2$.

$V C=p e{ }^{\star} R \star 2 \star 2 * H$

if (dpv.gt.0.4) go to 2004

$d p v=0.4$

$a a=(r m i * b o) * \star 2+r k i n * a p v * d e x t o / r k r m * \star 2$

$b b=0.5 * a p v * r k i n /(r k r m * * 2 * a a)$

$c c=(r k i n / r k r m-1$.) $/ a a$

$r 1 v=-b b+8 q r t(b b * * 2+c c)$

Page B-27 


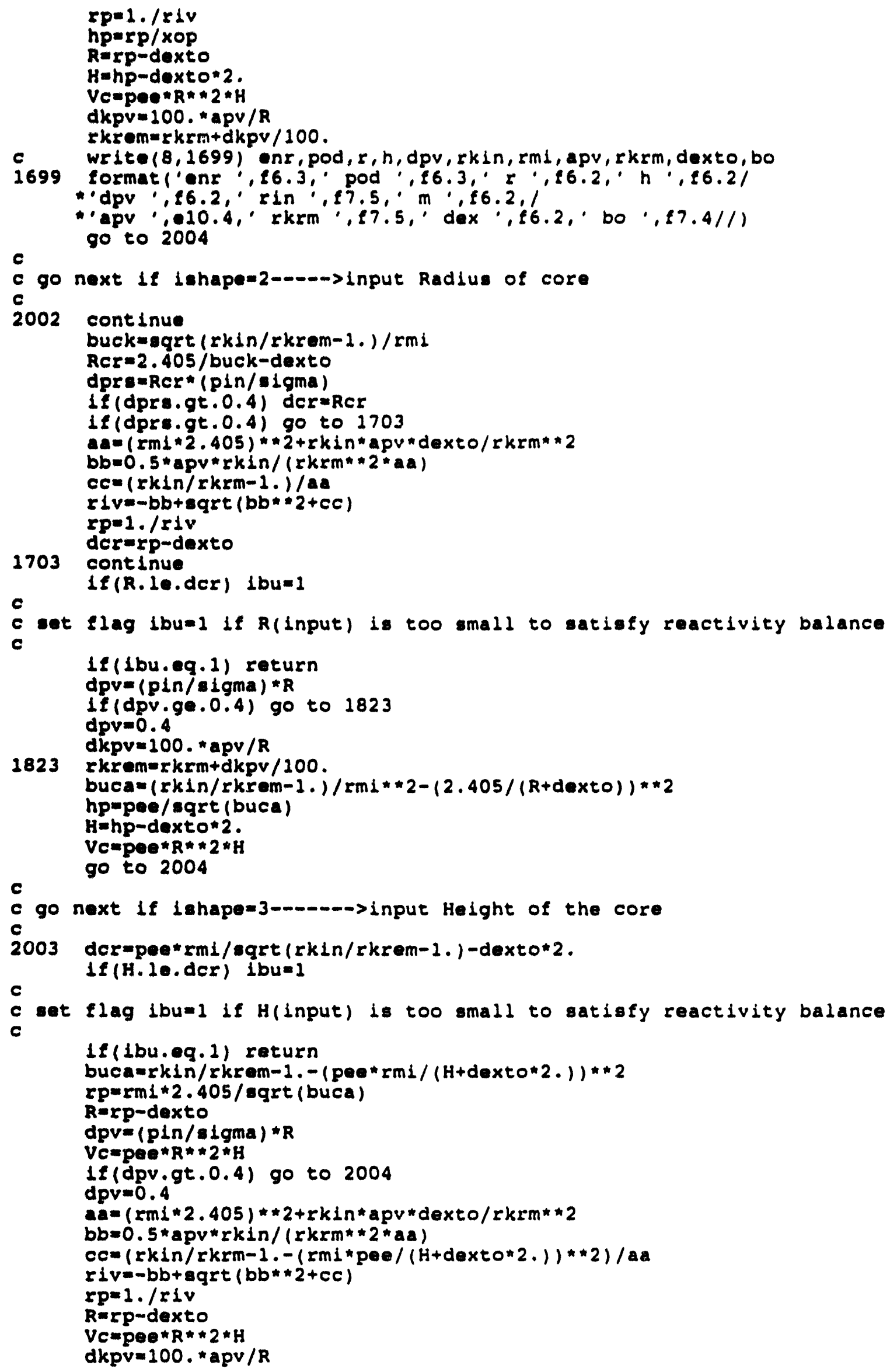




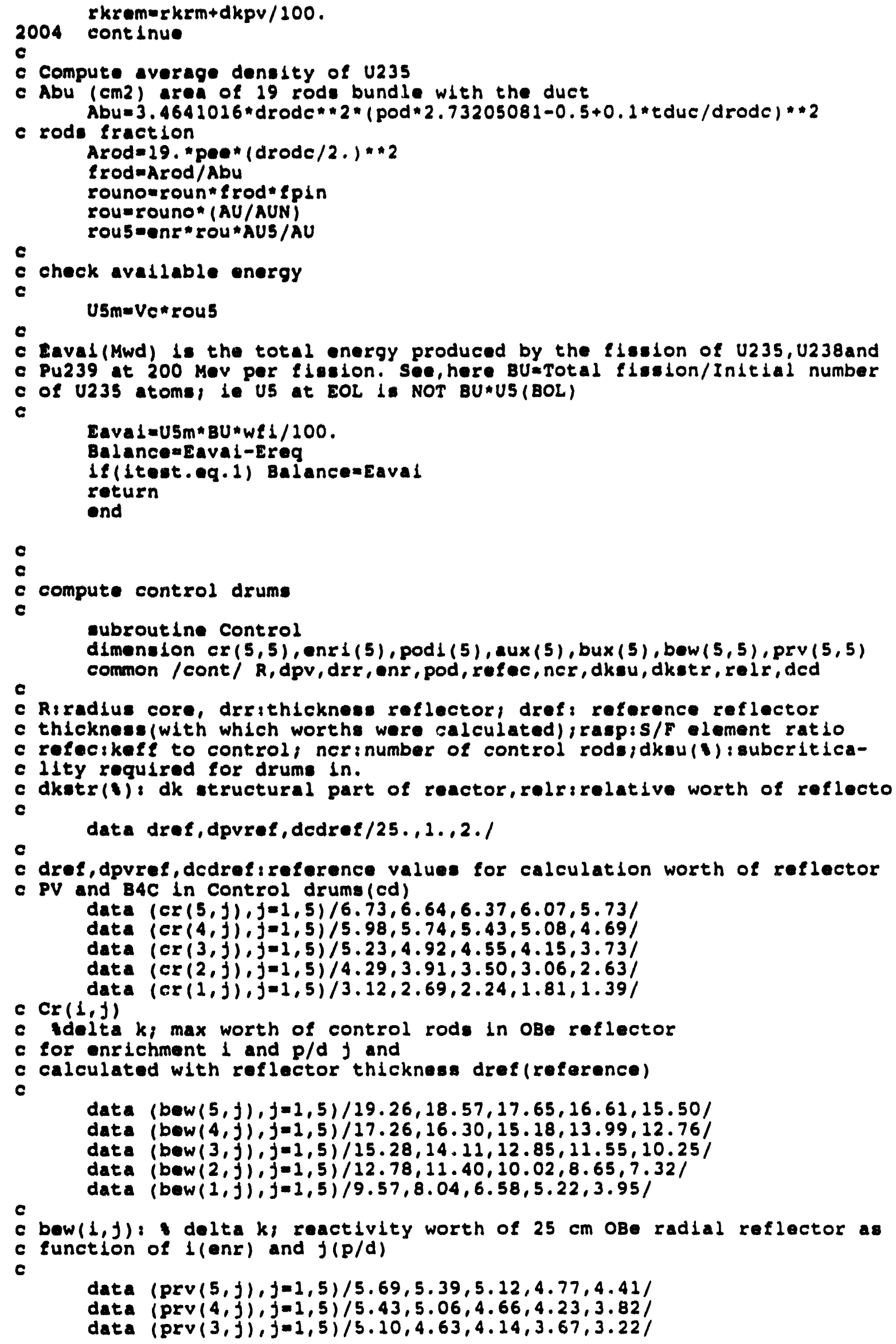

Page B-29 


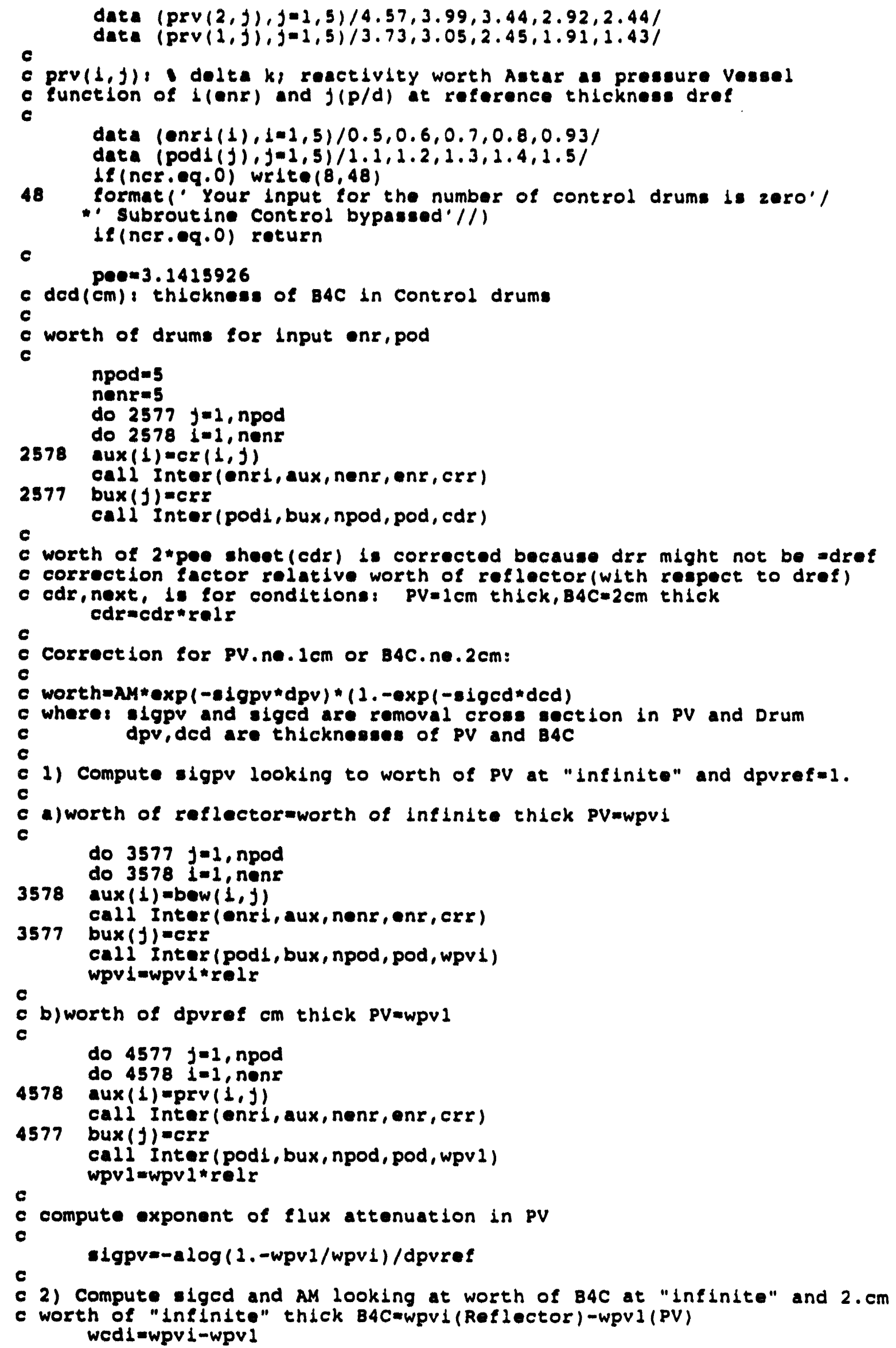




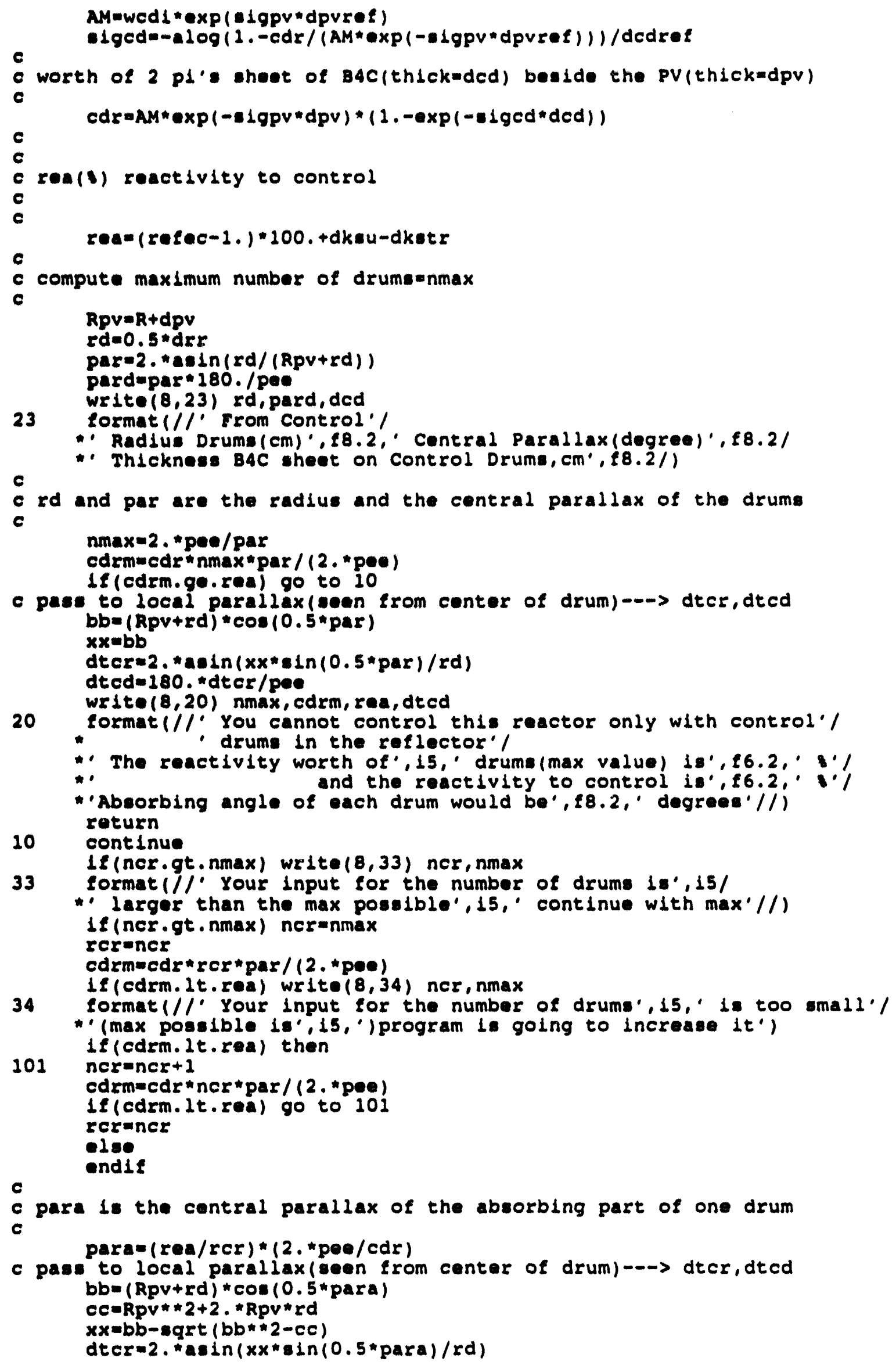

Page B-31 
$d t c d=180 . " d t c r / p e e$

write $(8,31)$ ncr, dted, rea

31

format (//15,' Drums with absorbing angle(d)', $\$ 8.2 .^{\prime}$ each'/ Control $d k(s)=,, f 8.2 / /)$

return

and

c

colve thermalhydraulic

nubroutine thermoli(lop)

real $4 \mathrm{k}$

dimension pow (51), prese (51), tbulk(51), ve(51), z(51), rj(51)

- tw(51), Tclad(51), Tfuel(51), Tclaa(51), Tfua(51), Tbuma(51)

c

common/thermo/ aln, nnodo, ifri, npin, pin, tin, tout, deltapa

*, Powt, rje, flowt, pod, tduc, Re

*, Hact, Pfacr, Daxi, fact, iprth, lopth, ichoose

* Pfacar, iboun, nrod, nbu, nrodbu, hodo

common /fric/ podf, hod, xx, drel, coefri

character*80 ain

$\operatorname{Tsa}(p q)=8143 . /(8 .-\operatorname{alog} 10(p 8 / 0.133322))$

$\operatorname{Paa}(t s)=0.133322 * 10 . *(8.00-8143 . / t 8)$

Tsa (ps) is saturation temperature of $L I($ In $K$ ) as function of $p s$ (KPa)

Pea(ta) is " pressure of Li(in KPa)" " of te(K)

data pee/3.1415926/

c

data drod, delad,dliner, dHe, TLim /0.64,0.0635,0.0127,0.0025,453.7/

data rpin/0.2413/

data $\operatorname{Tin} T a, \operatorname{TmUN} / 3269, .2903 . /$

cm diameter rod,clad, liner, He thickness

nnode: number of nodes:

iboun / $1,2 /$ boundaries conditions : read flow or $T$ outlet

ifri.ne.1: switch off friction factor

nclad: number nodes ineide clad

npin: number of nodes inside fuel

lcheck/0,1/ no,print some parameters to check them

pln(MPa), tin( $K$ ) inlet conditions

pod=p/d.

fact: factor to multiply the power

total power, $r j=(\mathrm{Kw} / \mathrm{cm} 2)$ ecale for plot heat rate

Vact(I) active volume of the core, ie Volume occupied by UN

tduc (mm) duct thickness; hod lead of the spacing wire(in unit of drod)

c

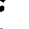

flowt $(\mathrm{Kg} /$ sec) : Total flow

Clowl,2,3(g/eec): Channel flow for cell types $1,2,3$

pin(MPa), tin( $K$ ) inlet conditions

total power: rje(Kw/cm2) scale for plot heat rate

c

npu=nnodo+1

Palge B-32 


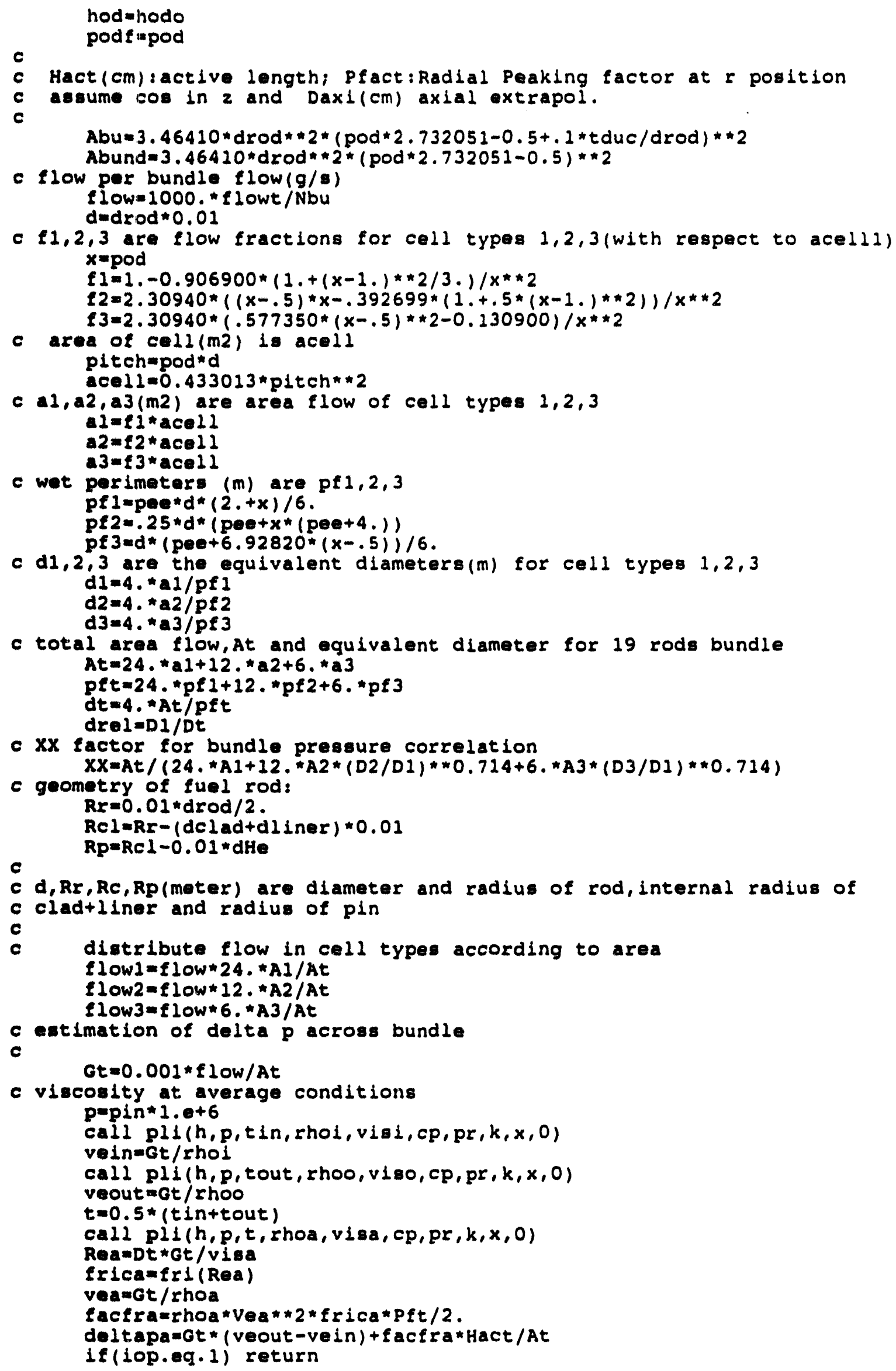


C

c

c

1 lop=l return after calculating 1 node estimation of delta $P$

dzet $=$ Hact / nnodo

Vact =nrod*pee*rpin**2*Hact $/ 1000$.

roave Powt *fact/Vact

write $(8,12)$ aln

format (a80)

write $(8,21)$ TLim, tin, drod, Powt, nrod, Nbu, nrodbu, flowt, Vact, fact

format (//" Thermodynamic profile of a liquid Li cooled channel'/

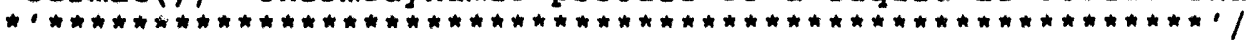

$*$ *

Remember li melts at', f8.2, $\mathrm{K} ! 1 ! !$

$* 1$

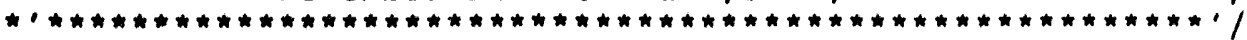

* Inlet temperature $(K)=0, f 10.21$

* Dlameter Rod $(\mathrm{cm})=', 7.21$

* Total Power $(\mathrm{Mw})^{\prime}$, f10.3,' No. Rods',110,' No.Bundles', i5, '1',13,' *rods/bundle)"/

* 'Total Flow $(\mathrm{Kg} / \mathrm{sec}) \cdot, \ldots 10.3 /$

*'Active volume (L of UN)', $f j 0.3 /$

* Factor that multiplies Power', $\$ 10.7 / / 1$ write $(8,162)$ roav, Hact

format ('Average Power Density $(\mathrm{MW} / \mathrm{L}(\mathrm{UN}))^{\prime}, f 8.4,^{\prime}$ Channel Length(cm)

$*, \ldots 7.2 / 1$

$A 1 C=A 1 * 10000$.

$A 2 C=A 2 * 10000$.

$A 3 C=A 3 * 10000$.

Atc $=$ At $\star 10000$.

$A l c r=A 1 c / 0.5$

$A 2 c r=A 2 c / 0.5$

$A 3 c r=A 3 c * 6$.

AtCr $=A$ AC $/ 19$.

pe 1 c=pf $1 * 100$.

pf2c=p\& $2 * 100$.

p $f 3 c=p \in 3 * 100$.

pftcopft* 100 .

$d I c=d I * 100$.

d2 $c=d 2 * 100$.

$d 3 c=d 3 * 100$.

$d t c=d t * 100$.

$f t=A t c /$ Abund

$n 1=24$

$n 2=12$

$n 3=6$

write $(8,190)$ pod, hod

format $/ / / /$ FLOW CONDITIONS: '//

* Pitch/drod', f8.3,' Lead wire spacer/drod', $f 8.3 / /$

* Cell type (per bundle) ', 11x, $1^{\prime}, 11 x, \cdot 2^{\prime}, 11 x, \cdot 3^{\prime}, 6 x$, Bund $^{\prime} e^{\prime} / /$ )

write $(8,191) \mathrm{nl}, \mathrm{n2}, \mathrm{n3}, \mathrm{flow1}$, flow2, flow3, flow, $f 1,\{2, \in 3, f t$,

$A 1 C, A 2 C, A 3 C, A t C$

A1cr, A2cr, A3Cr, Atcr, pf $1 c, p \in 2 c$, pf $3 c$, pftc, $d 1 c, d 2 c, d 3 c, d t c$,

format 1 $x X, d r e l$

* Number of Cells

* Elow $(\mathrm{g} / \mathrm{s})$ Total., $4 f 12.4 /$

* Flow Area (*) $\because 4 \notin 12.4 /$

* Flow Area (cm2), per cell', $4 f 12.41$

* Flow Area (cm2), per rod, $4 f 12.4 /$

* Wet Perimeter (cm) Total',4f12.4/

* Effective Diameter (cm) ?, $4 f 12.4 / /$

* (*) $1,2,3$ in units of cell 1 area $/ /$

* Parameters of the bundle for friction correlations:

* $X=1, f 6.3, \cdot$ Del/DT=', f6.3//)

if (iboun.eg.1) write $(8,237)$ tout

if(iboun.eg. 2) write $(8,238)$ tout

Page B-34 


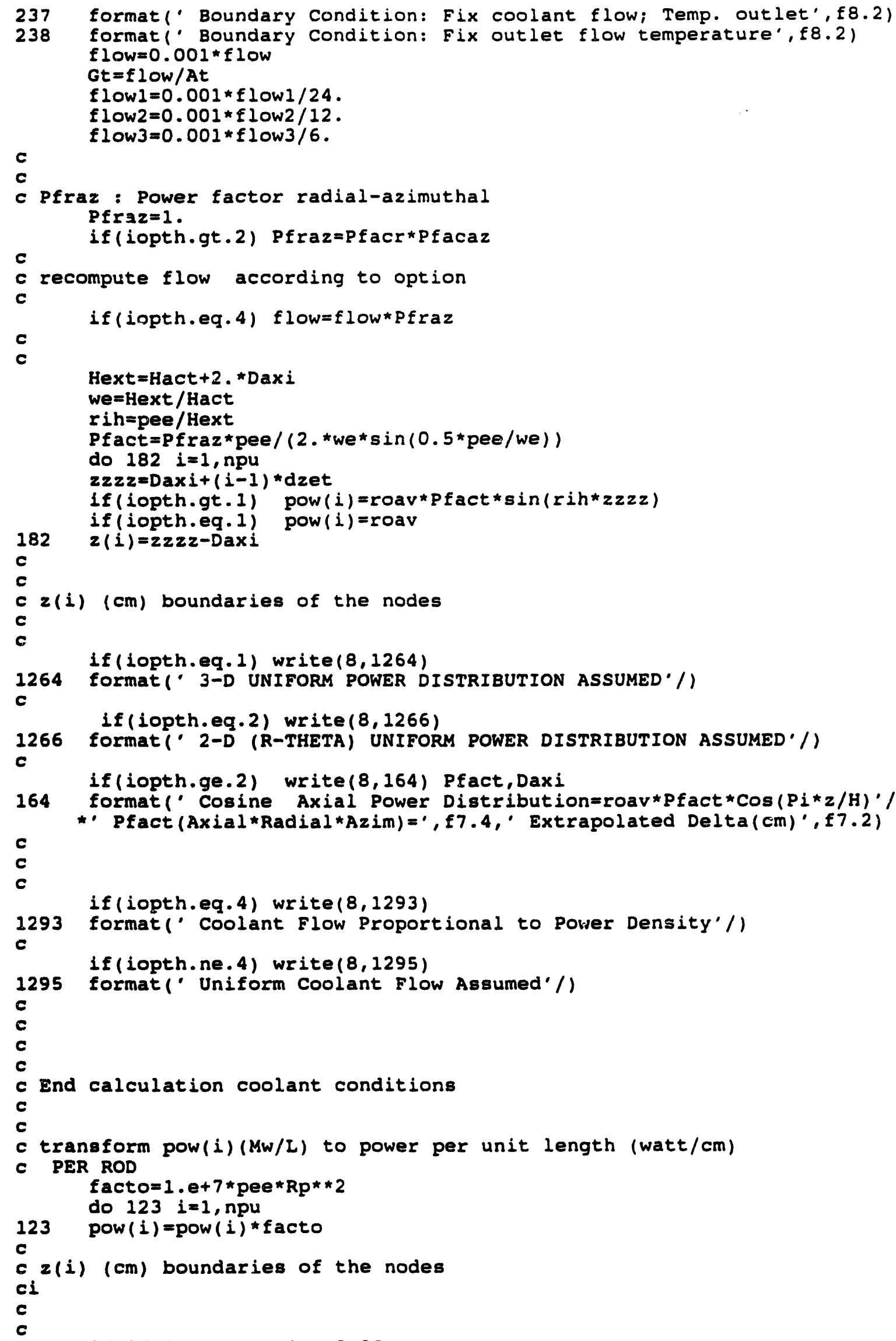




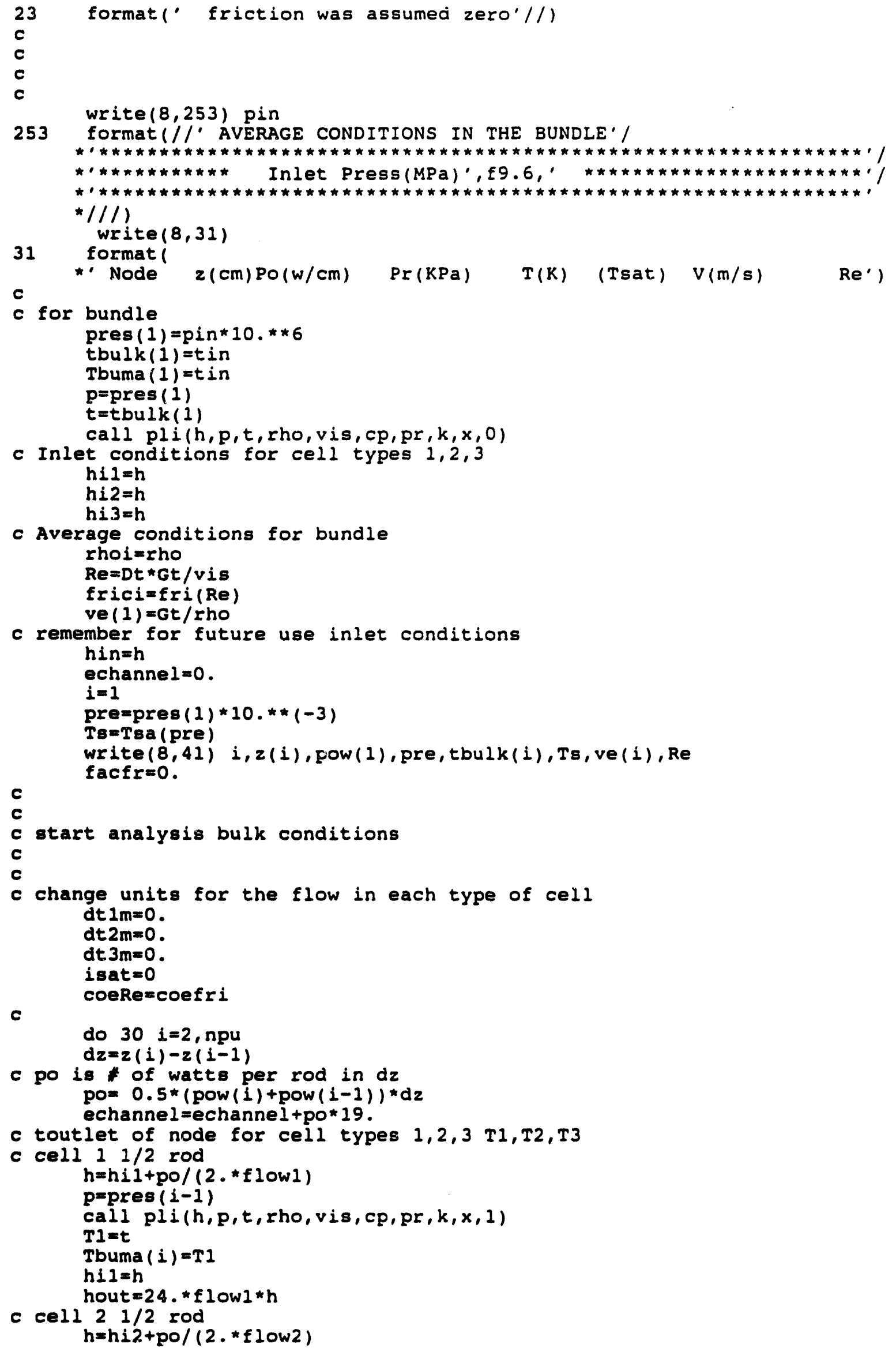




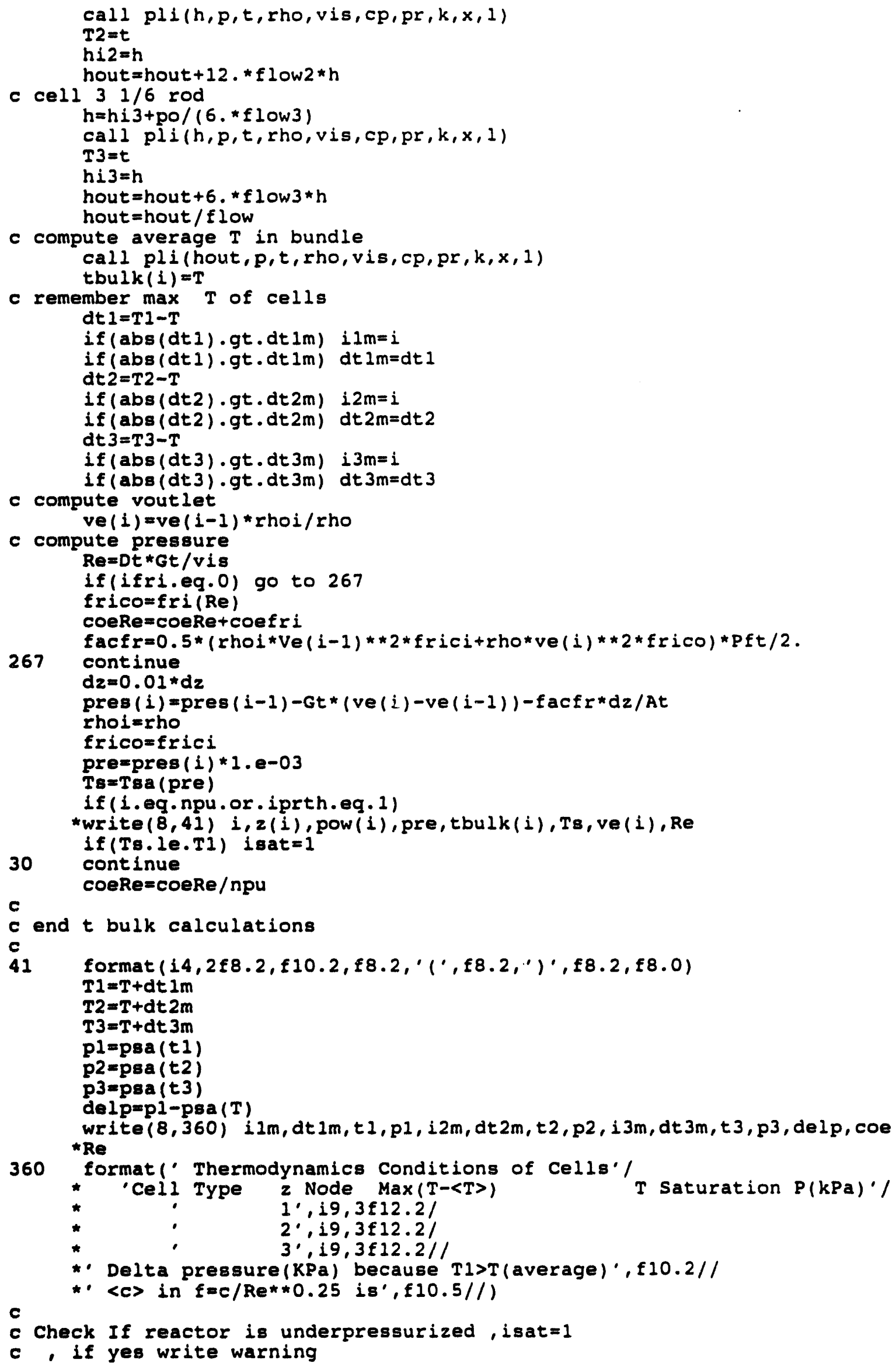


if (isat.eq.0) go to 350

write $(8,351)$ Ti, Ts

351 format('Although, in a BULK sense, the pressurization is correct'/

* the temperature of cell type 1 ', f10.2/

* reaches or passes boiling temperature', f10.2/

* so some flushing is possible."/

*'You might:'/

*'1) Increase $p$ at inlet./

*'2) Reduce $T$ outlet(if iboun=2), or increase flow(if iboun=1)'/

*3) Reduce T inlet./

* 4) Assume 2D uniform power distribution."/

* 5) Assume flow grading (iopt $h=4$ ) \%

* 6) Use larger bundle (not available yet) $/ / /$ )

if (ichoose.eq.1) stop

2515 write $(8,2516)$

2516 format $1 / 1$

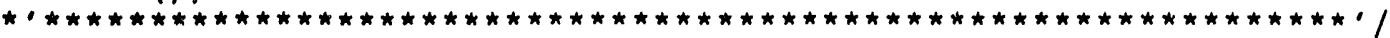

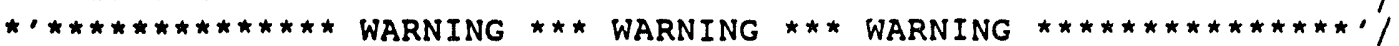

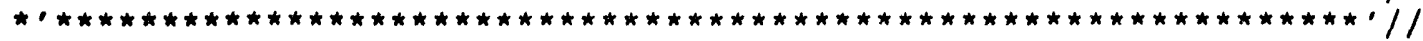

* Despite previous caveats, Program continues with nominal values'/

$\star$ )

350

continue

c

c calculation wall temperature and rod temperatures for MAX HEATED ROD

c IN bundle which is type 1 cell

c

c Initialization of function RNu

Dummy $=\operatorname{RNu}(\operatorname{Re}, 1,1, \mathrm{pod})$

dg $=$ dHe*0.01

GI $=$ flow $1 / \mathrm{al}$

c

c loop on 2 nodes

c

have $=0$.

TTa $=0$.

$T U N=0$.

do $302 i=1$, npu

p=pres (i)

$t=t$ buma $(i)$

c

C SEE: we have chosen the highest $T$ (cell I) Tbuma(i)

call $p l i(h, p, t$, rho, vis, $c p, p r, k, x, 0)$

Re $=G 1 * D 1 / v i s$

c define Peclet number

$P e=R e * P r$

$\mathrm{RNu} s=\mathrm{RNu}$ (Re, $\mathrm{Pe}, 0$, pod)

hCo=RNus*k/Dt

have = have+hco

c rjqwatt $/ \mathrm{m} 2$

$r j q=$ pow $(i) \star 100 . /($ pee*0.01*drod)

c $r j(i)=K w / \mathrm{cm} 2$

$r j(1)=r j q * 10 * *(-7)$

c Qh=watts $/ \mathrm{m}^{3}$

$\mathrm{Qh}=100 .{ }^{*}$ pow $(1) /($ pee*Rp**2)

$T w(i)=T+r j q /$ hco

c From Twa to Tfuel

c Clad+liner

cclad $=$ Rkclad ( Twa)

c see only one node for the clad

c

$\operatorname{Tclad}(i)=\operatorname{Tw}(i)+r j q * \operatorname{Rr} * a \log (\operatorname{Rr} / \operatorname{Rcl}) / \operatorname{cclad}$

$\operatorname{Tclaa}(1)=\operatorname{Tw}(i)+r j q * R r *(0.5-a \log (R r / R c l) * R c l * * 2 /(R r * \star 2-R c l * \star 2)) /$

*clad

if (Tclad (i).gt.TTa) Tra=Tclad (i)

c delta $T$ across he gap

Page B-38 
$T g=T c l a d(i)$

hg=Rkgap ( $T g, d g)$

Tgap $=$ Tclad $(i)+r j q / h g$

c temperatures inside pin, npin nodes

$\mathrm{dr}=\mathrm{Rp} / \mathrm{npin}$

Tinl $=$ Tgap

$\operatorname{rinl}=R p$

Tf $a=0$.

do 3021 in=1, npin

Cfuel=RkUN (Tinl)

rout $=$ rinl $-d r$

Tout $=$ Tinl $+0.25 * Q h *($ rinl $* * 2$-rout $* * 2) /$ Cfuel

Tfa $=\mathrm{Tfa} a .5 *($ Tout $+\mathrm{Tinl}) *($ rinl $1 * 2-$ rout $* \star 2)$

Tinl=Tout

rinl 1 rout

3021 continue

Tfuel (i) = Tout

if (Tout.gt. TUN) TUN=Tout

Ifua (i) $=$ Tfa/Rp* *2

302

continue

c

have $=$ have $/(10000$. npu $)$

$c$ end loop on 2 nodes pin

$\operatorname{drd}=10$. *drod

$d c l=($ dclad $+d l$ iner $) * 10$.

deHe $=1000 . * d g$

dpin=drd-(dcI+deHe $) * 2$.

write $(8,978)$ drd, dcl, deHe, dpin

978 format(//. From the Coolant Channel to fuel pin temperature'/

* Rod Diameter(mm): ', $f 8.4 /$

* Clad+Liner Thickness( "):', $\$ 8.4$ /

* He gap " ( "):', f8.4/

* Fuel Pin Diameter ("): ", $f 8.4 / 1$

* CONDITIONS FOR MAX HEATED ROD OF THE BUNDLE(TYPe 1 cel1)') write $(8,976)$

976 format $\left(/ / /^{\circ}\right.$ Node $z(\mathrm{~cm}) \mathrm{J}(\mathrm{KW} / \mathrm{cm} 2)$ Twall(K) Tclad(K) TcladAv

* Tfuel(K) Tfuelav $/ / /$ )

do $2583 i=1$, npu

if (i.eq.1.or.i.eq.npu.or.iprth.eq.1)

*write $(8,977) i, z(i), r j(i), t w(i), \operatorname{Tclad}(i), \operatorname{Tclaa}(i), \operatorname{Tfuel}(i), T$ fua(i)

2583 continue

977 format $(15, f 10.2, £ 10.5,5 f 10.2)$

c calculation of axial averages

C

if(TTa.ge.TmTa.or.TUN.ge.TmUN) write(8,2633) TTa,TUN, TmTa, TmUN

format 1

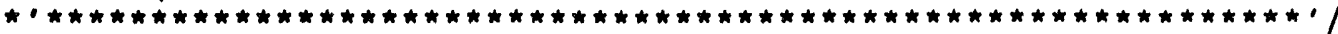

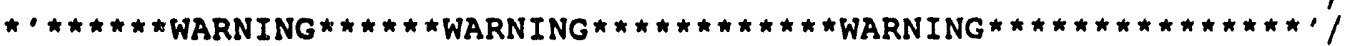

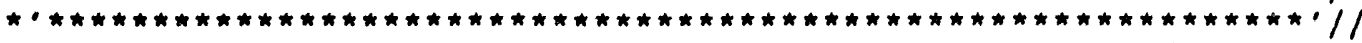

* Max. Temp(K) clad is',f9.2,'Max. Temp(K) UN is', f9.2/

* Ta melts at $(K) \quad, f 9.2$, ' UN melts at(K) ', f9.2/

* Relax inlet, outlet conditions or assume uniform power or'/

* flow grading $/ / 1$

zave0

thav $=0$.

phav $=0$.

twa $=0$.

tca $=0$.

$t f a=0$.

do $1436 i=2$, npu

$d z=z(i)-z(i-1)$

$z a v=z a v+d z$

phav $=$ phav $+0.5 *($ pres $(i)+$ pres $(i-1)) * d z$

$t w a=t w a+0.5 *(t w(i)+t w(i-1)) * d z$

$t c a=t c a+0.5 *(t c l a a(i)+t c l a a(i-l)) * d z$

$t f a=t f a+0.5 *(\operatorname{Tfua}(i)+\operatorname{Tfua}(i-1)) * d z$ 
thav $=$ thav $+0.5 *(t b u l k(i)+t b u l k(i-1)) * d z$

roin=roout

thavethav/zav

phav $=$ phav $/ z a v$

$t w a=t w a / z a v$

$t c a=t c a / z a v$

$t f a=t f a / z a v$

pre $=$ phav $* 0.000001$

Write $(8,1486)$ pre, thav, twa, have, tca, tfa

1486

Format (//' Axial Average Conditions in the Channel'/

* Coolant Pressure(MPa)', f8.3,' Temperature(K)', f9.3/

* Wall Temperature $(K)^{\prime}, f 9.3,^{\circ}$ Heat $T r a n s f e r(w / c m 2 K)^{\prime}, f 8.2 /$

* Clad Temperature(K), $f 9.3 /$

*

dh=hout - hin

Fuel Temperature $(K)^{\prime}, f 9.3$ )

$d q=e c h a n n e l / f l o w$

$d k i n=0.5 *($ ve $(n p u) * * 2-v e(1) * * 2)$

rel $=\mathrm{dkin} / \mathrm{dq}$

bal $=(d h+d k i n) / d g$

write $(8,987) \mathrm{dq}, \mathrm{dh}, \mathrm{dkin}, \mathrm{rel}, \mathrm{bal}$

987 format (//, HEAT BALANCE FOR THE BUNDLE'//

* 'dQ: heat to coolant (joule/kg)',e14.5/'dH: change of enthalpy

1 (joule/kg)', e14.5/'dKin change kinetic energy (joule/kg)', el4.5/

$2^{\prime}$ relative dKin/dQ',f10.6/' Balance (dH+dKin)/dQ',f14.7)

return

end

C

c friction factor for bundle cooled by liquid metal

function fri(Re)

common /fric/ pod, hod, $x x$, drel, coefri

c where Re is the Reynold's number

c Novendstern correlation, page 131 ANS book about LMFBR

if (Re.le.1000.) then

fri $=21$. $/ R e$

coef $x i=21 . / R e * * 0.75$

else

coefri $=(1.034 /$ pod**.124+29.7*pod**6.94*Re**.086/hod**2.239)**.855

coef $r i=0.079 * \operatorname{coef} r i * x x * 1.75 / \mathrm{d}$ rel $l * 1.25$

fril=coef $r i / R e \star \star 0.25$

endif

return

end

c

c

subroutine bisect $(x 1, \times 2, x a v, f 1, f 2, f a v, i w a)$

if $(f 1 * f 2)$.le.0.) go to 11

write $(8,1)$

1 format $(/ /, f 1 * £ 2>0 . \cdot / /)$

iwa $=1$

return

11 iwa $=0$

if ( $(f a v * f 1) .1 e .0$.$) go to 10$

$f l=f a v$

$x 1=x a v$

return

$10 \quad £ 2=f a v$

$\times 2=x a v$

return

end

c 
c Nusgel Number for one Phase Flow of liquid metal along fuel elements

C

function RNu(Re, Pe, ini, pod)

dimension $B(4), x(4), \operatorname{cc}(6), \times x(6)$

data $(b(1), i=1,4) / 7.25,10.65,12,12.85 /$

data $(x(1), 1=1,4) / 1.1,1.2,1.3,1.4 /$

data $(c c(j), i=1,6) / .00293, .00253, .002078, .001607, .001194, .000862 /$

data $(x \times(1), 1=1,6) / 1.01,1.2,1.4,1.7,2.2,3.1$

$c$ Where $R e$ and $P e$ and Reynolds and Peclet numbers evaluated at bulk

c conditions, ini=1 initialize according pod and return. ini=0 compute

c Nussel number after the initialization. Correlation from page 189 ANS

c book about LMFBR's

if (ini.ne.1) go to 10

call Inter $(x x, c c, 6$, pod, $c)$

if (pod.1e.1.35) then

al $=0.80$

call Inter $(x, b, 4$, pod, $c 1)$

$c 2=0.025$

else

$\mathrm{al}=0.86$

$c 1=6.66+3.126 *$ pod $+1.184 *$ pod * *2

$c 2=0.0155$

10

endif

Pr $=$ Pe/Re

eon $=C * R e * \star 0.915$

psi=1. $-1.82 /($ Pr*eon**1.4)

if (psi.1t.0.) psi=0.

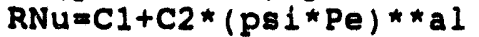

return

end

c

c Function: Thermal Conductivity of UN

c Source: Alkasys Manual

c

Function RkUN(Te)

c $T(K)$ RkUC (watt/mK)

c

RKUN $=24$.

Return

c

End

c

c Function: Thermal Conductivity of He gap

c Source: Alkasys Manual

c

Function Rkgap (T,d)

c $T(K), d(m)$ Rkgap (watt/m2K)

C Rkgap=k(He)/d(He), $k(\mathrm{He})$ from ANS book about gas cooled reactors Rkgap $=0.002774 * T * * 0.701 / d$ Return

c

End

$c$

c Function: Thermal Conductivity of Astar clad and $W$ liner

c Source: Alkasys Manual

c

c

Function Rkclad(Te)

c $T(K)$ RkUC (watt/cmK)

c

Rkclad $=57$.

Return

End 


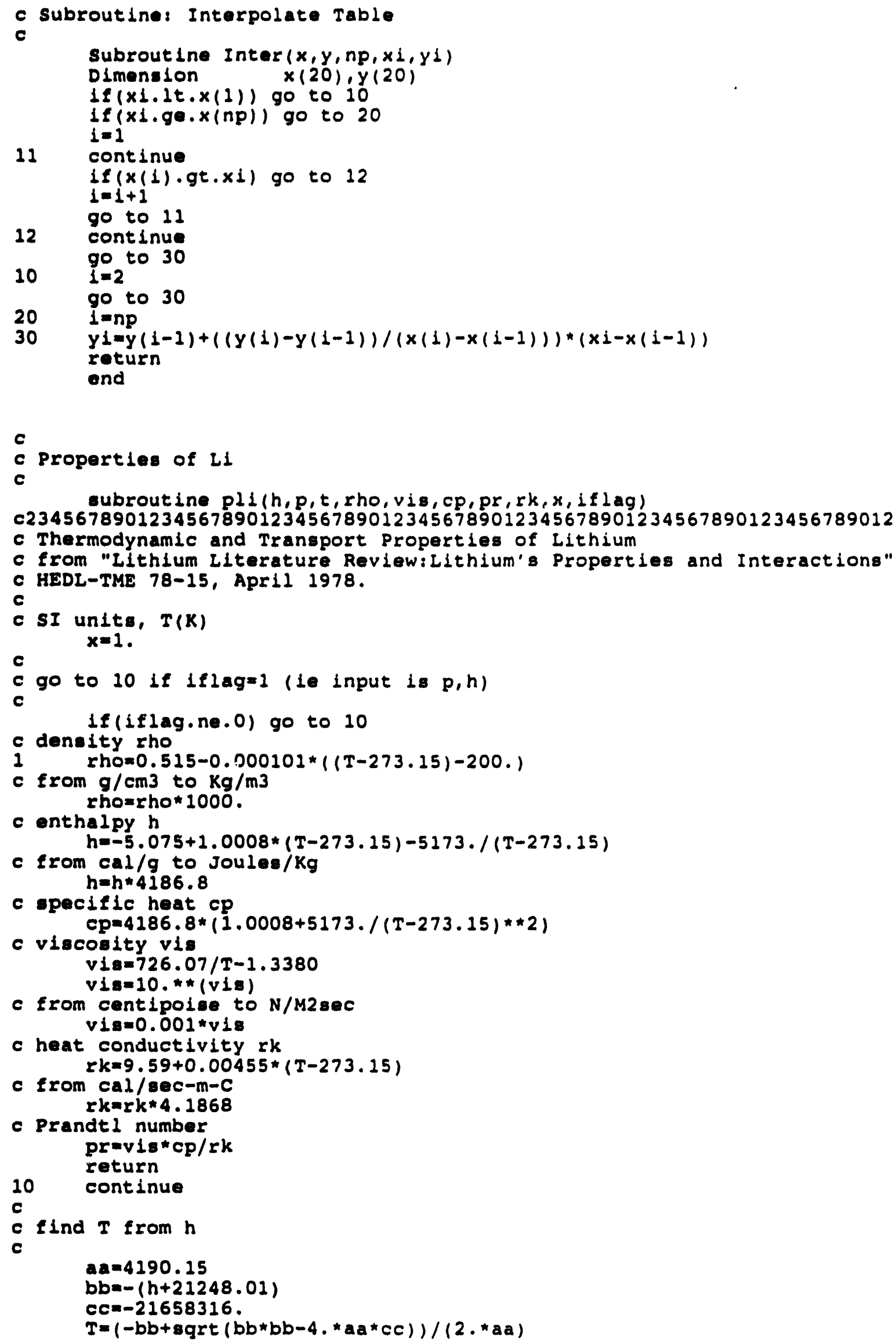


$T=T+273.15$

go to 1

end

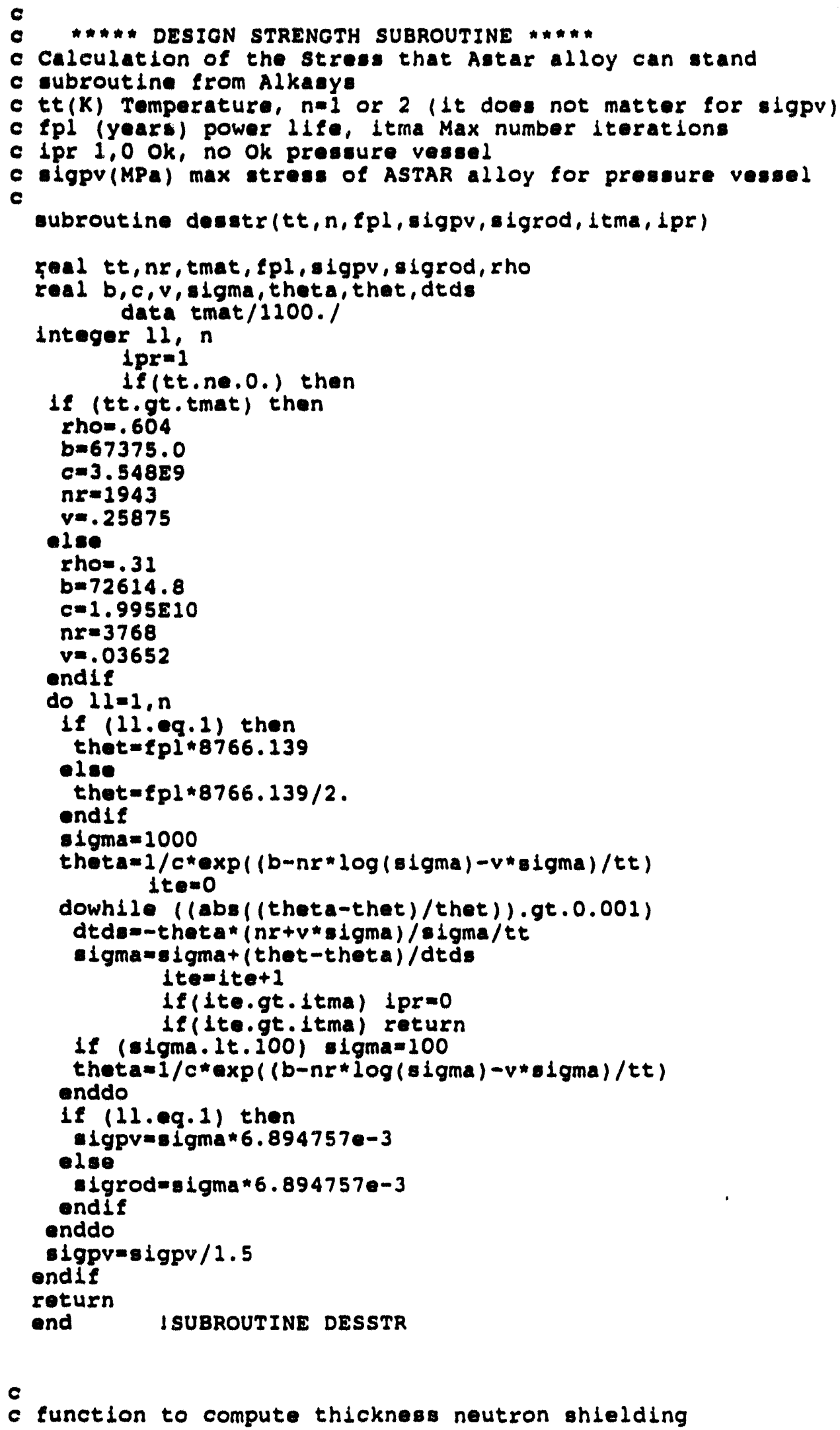

c

c function to compute thickness neutron shielding 
Punction shieldn (fluln, iluout, ish, tw, lgam)

common /crose/ AA,BB, signe, Lehin, lehtg

$c$

c Fluin, fluout, fast fluxes in/out; lsh=1, LiH+Ss

c Loh=2, B4C, tw(cm) gamma shielding (Tungstene)(if lgam.ne.0)

c

data romL 1 , ramH, romFa, romw, raniBC

* $/ 1.01,1.00,1.98,3.13,5.1 /$

c removal crose ection for fast noutrons of $L L, H, F e, W, B 4 C$

c

data Av, roLiH, rOW, rOBC, roFe, roL Le, ALLH, AFe, ABC, AW

- $\quad 10.602202,0.82,19.3,2.52,7.874,1.024,7.95,55.85,55.26,183.85 /$

IgW=romW"Av"roW/AW

ishin=1

go to $(10,20), 1$ sh

$X L L H=($ rOLLEs-rOFe $) /($ rOLLH-rOFe)

$x F=1,-x L \perp H$

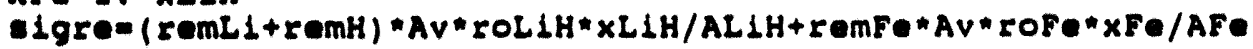

go to 30

20 lgre=remBC*Av*roBC/ABC

30 anmalog(fluin/Eluout)/sigre

Le(a.ie.0.) ishin=0

$\mathrm{bb}=\mathbf{g} \mathbf{g W} / \mathrm{gigre}$

elone-eigre

shieldn=a $a-b b * t w$

return

and

c

C Subroutine to calculate Gamma source

c

Subroutine Gameo ( $\$ n$, eogam, PrU, Evr)

common / Conce/ cOBe, cOTa, cOU5, cOU8, cOL1, cOW, cORe, cOHE, cOHe, cON, cOO

c

c Input: $8 n(1 / 8)$ : neutron production, fvr: volume fraction of reflector

c Output:

c sogam(1): Gamma source between "O" and I Mev

c (2):

(3):

(4):

(5):

(6):

(7):

1 and 2

2 and 3

3 and 5

5 and 7

7 and 9

$>$ around $9 \mathrm{MeV}$

and PrU(): fractions of gammas produced in $U$

Dimension Sogam (7), PI(7), $\mathrm{PP}(7), \mathrm{GL}(7), \mathrm{GO}(7), \mathrm{GTa}(7), \mathrm{GBe}(7)$,

$* \operatorname{OU}(7), \operatorname{GRe}(7), \mathrm{GHE}(7), \mathrm{GW}(7), \mathrm{GN}(7), \operatorname{PrU}(7)$

c

C PE: Prompt Fiseion Gammas; Fp: Flesion Products Gammas(Both, Per Fies)

c ONL to GCriCaptures Gammas (per Event) for Indicated Elements Ni,..Cr

C Data from ANL-5800 page 631. For O: the 8 Mev released for the capture

$c$ of one neutron was uniformly distributed in range 0-10 Mev.

c seescapture in 0 is almost 0.0 , Remember B10 has ( $n$, alpha) rather $(n, g)$ Data (PI $(1), 1=1,7) / 3.1,2.11, .73, .26, .0428,0 ., 0.1$

Data (Ep (1), 1=1,7)/9.03,1.137,.4,0.,0.,0.,0.1

Data $(0 L 1(1), 1=1,7) / 0 \ldots, 0,0,0,0.4,0.6,0.1$

Data (GTa $(1), 1=1,7) / 1.37,0.99,0.66,0.55,0.05,0 ., 0.1$

Data (OW $(1), i=1,7) / 0.68,0.82,0.59,0.53,0.15,0.005,0.1$

Data (GRe $(1), 1=1,7) / 1.24,0.88,0.62,0.51,0.105,0 ., 0.1$

Data (OHf (1) $1=1,7) / 1.37,1.37,0.85,0.52,0.12,0.005,0.1$

$\operatorname{Data}(O N(1), 1=1,7) / 0.0,0,0 ., 0.54,0.11,0.15,0.12 /$

$\operatorname{Data}(G O(1), 1=1,7) / 0.16,0.16,0.16,0.32,0.32,0.32,0.16 /$

Data $(G B e(1), 1=1,7) / 0 \ldots 0,0 \ldots .54, .73, .0, .0 /$

Data (GU (1), $i=1,7) / 2.54,1.78, .91, .34, .0,00,0.041 /$

Data Rnu/2.52/

Data scLi, scTa, scW, scRe, schf, scN, sco, scBe, scU5, scU8

Pange B-44 


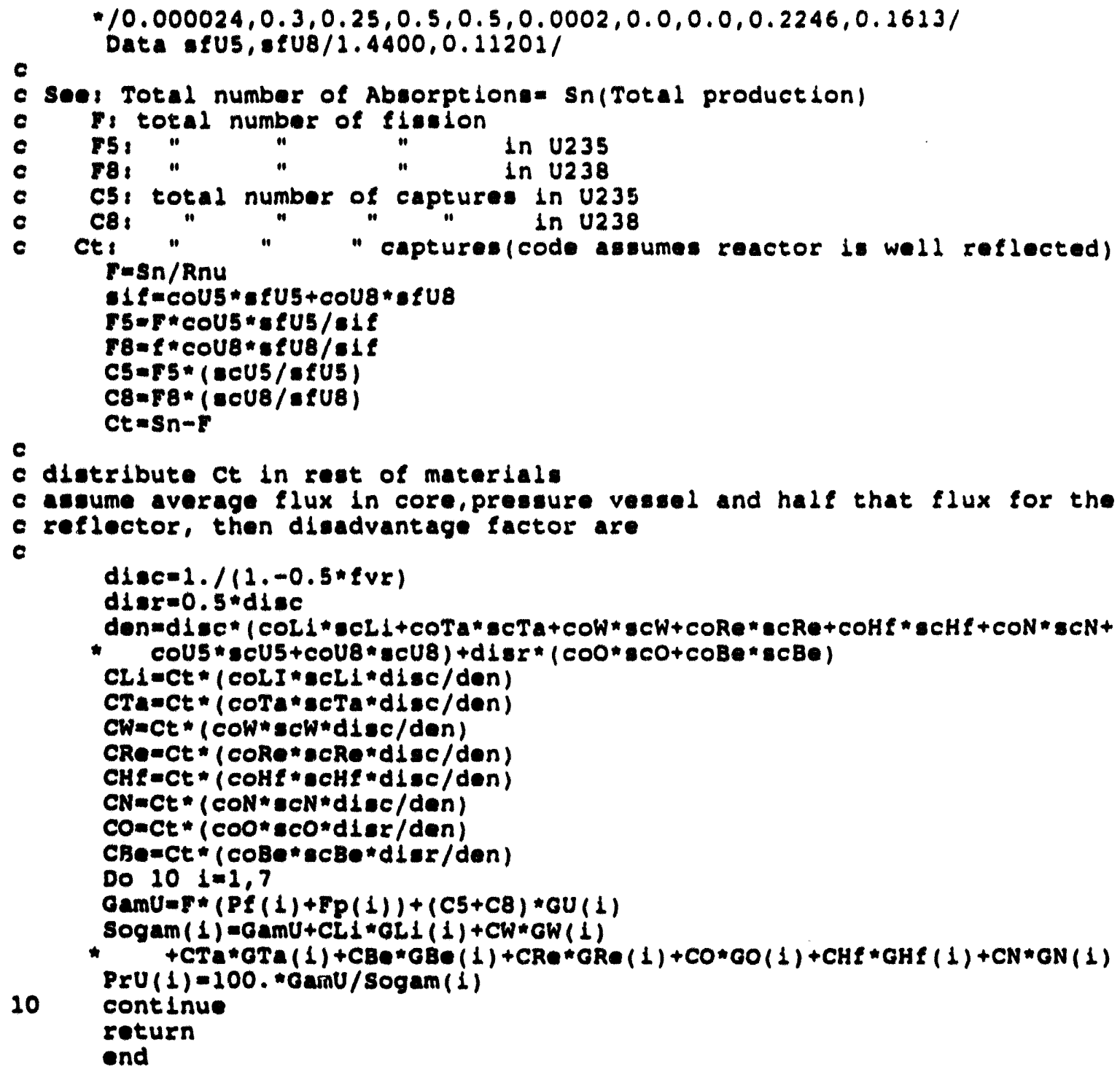

c

c

subroutine shieldga (Sogam, Dose, Dosea, Dosew, tiempo, tgam, ten, Igam, *ishn, iten)

Common /CROSs/AA, BBB, signe, ishin, ishig

c

C shleldga: Computer gamma shielding

c Sogam(1),1=1,7 Gamma Source (photons/sec); Dose(rad): Integrated

c Dose at load area; Dosew(rad): Dose without the shielding

c Dosea: dose for tgam

c igamitlag if - I probleme with iteratione

c $\operatorname{Tgam}(\mathrm{cm})$ : Thlckness of Tungsten

c ten (cm) thickness of $n$ shielding, ishn=1, LiH, lohn=2, B4C

c iten $/ 0,1 /$ /ten is input, ten is iterated by this subroutine/

c tgam: iterated, if necensary, to have Dose=input

c Index (1): Gamma Processes between "O" and I MeV

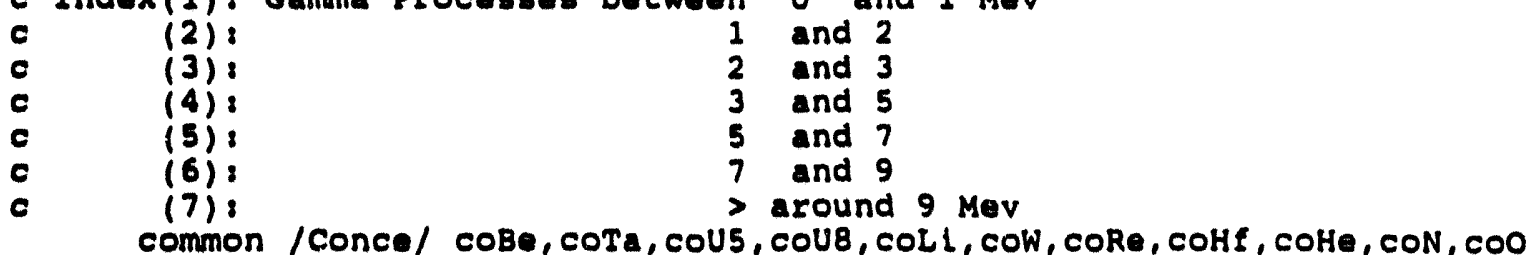

Page B-45 
c

c

c GBE...OU are the total mass attenuation coefficients $(\mathrm{cm} * 2 / \mathrm{g})$

c for the 7 gamma groups. $L 1(z=3)$ and $B(z=5)$ are lumped with $B e(z=4)$

c $T a(z=73), R \in(z=75)$ and $H E(z=72)$ are lumped with $W(z=74)$

c $N(z=7)$ is lumped with $O(z=8)$

$\mathbf{c}$

c

c

c

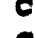

$$
\text { c }
$$

c

-

data $(b(1, j), j=1,7) / 8,5,5,4.5,3,8,3,1,2.3,2.25 /$

date $(b(2, j), j=1,7) / 10 ., 8,5,5,3,75,2.9,2.35,2.0 /$

data $(b(3, j), j=1,7) / 3.3,2.9,2.7,2.05,1.2,7, .6 /$

data $(b(4, j), j=1,7) / 1.65,2.45,2.15,1.65, .96, .67, .5 /$

data $(a 3(1, f), j=1,7) / .11, .082, .074, .066, .064, .062, .060 /$

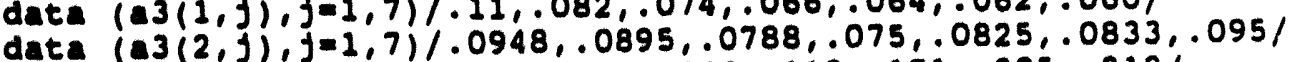

data $(a 3(3, j), f=1,7) / .043, .069, .086, .118, .171, .205, .2121$

date $(a 3(4, j), j=1,7) / .032, .045, .097, .123, .175, .204, .214 /$

date $(14(1, f), f=1,7) / .044, .093, .116, .130, .152, .150, .128 /$

data $(a 4(2, j), j=1,7) / .012, .04, .07, .082, .075, .0546, .0116 /$

data $(a 4(3, j), j=1,7) / .148, .188, .134, .070, .0, .052, .144 /$

date $(04(4, j), j=1,7) / .296, .178, .103, .064, .059, .067, .08 /$

data $(2 b(1), 1=1,4) / 13, .26, .74, .82 . /$

c

c

c

c

c

c

c

date $(\operatorname{ch}(1), 1=1,7) / 4 \cdot 5 e+5,5 \cdot 5 e+5,7 \cdot e+5,8 \cdot e+5,8.5 e+5,9 . e+5,1 \cdot e+6 /$

rh(1) Gamma Flux Mov/cm2sec that produces 1 Roentgen/hour

data $(\operatorname{Ega}(1), 1=1,7) / 0.5,1.5,2.5,4, .6,8, .10 .1$

ga (1) Mov Average Energy of gamma group 1

date ABe, ATa, AW, AO, AHs, AU5, AU8, AL1, ARe, AN1, AV, ROW

$* / 9.0122,180.948,183.85,15.9994,178.49,235.044,238.0508,6.939,186,2$

$\star, 14.0067,0.602202,19.3 /$

data $2 B=, 2 T a, 2 W, 2 U$, pee, $2 L 1,2 B O, 2 R e, 2 H E, 2 N, 20$

$* 14, .73 \cdot .74, .92, .3 .141593,3 ., 5,75 \ldots, 72 \ldots 7 ., 8.1$

data rol 1 , roBC, Ross, RoLis $/ 0.82,2.92,7.874,1.024 /$

lgam=1

1shig=1

XLLH= (ROLIS-ROSS) / (ROLLH-ROSS)

$x S S=1 .-x L \perp H$

c Attenuation*Bulld Up factor for $N$ shield(which also shield gammas)

c

do $101 j=1,7$

go to $(111,222)$, Lehn

c Iohn=1 LLH+SS

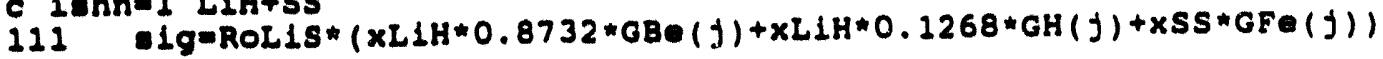

Paige B-46 


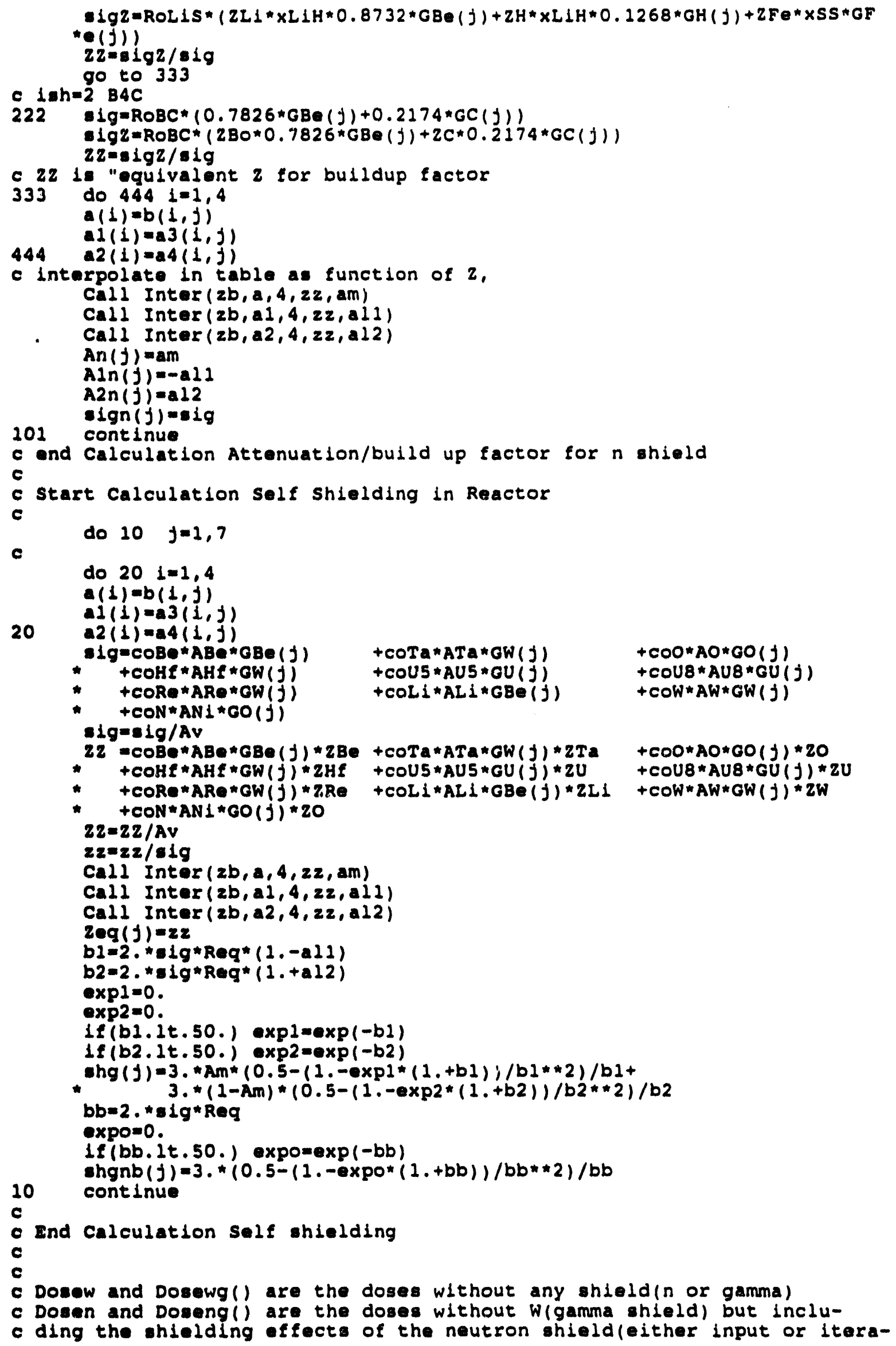


c ted

$t$ Ime=t Lempo/3600.

Dosew 0 .

Dosen $=0$.

if (iten.eg.1) ten=AA

if (iten.eq.1. and.ishin.eq.0) ten $=0$.

do $30 \quad 1=1,7$

$\tan =\operatorname{sign}(1) * \operatorname{ten}$

$\operatorname{sh} 1 n=\exp (-\tan ) *(A n(1) * \exp (-\tan * A 1 n(1))+(1,-A n(1)) * \exp (-\tan * A 2 n(1))$

*

Dosewg $(1)$-t ime*Sogam (1)*shg(i)*Ega (i)/(4. *pee*dist**2*rh(1))

Doseng $(1)=\operatorname{sh} 1 n \star D o s e w g(1)$

30

Dosew=Dosew+Dosewg (i)

Dosen-Dosen+Doseng (1)

if (Dosen. le. Dose) ishig=0

if (iohig.eq.0) tgam $=0$.

if (iohig.eq.0) return

c

c Roturn if there is no need for gamma shield (thick $w=0$. )

$t \mathfrak{l}=0$.

Dosel=Dosen

f $1=$ Dose1-D08e

c starts iteration on tgam:

c Firat look for

c t2 such that Dose $(t 2)<D o s e$

c

$1 t=0$

Big=GW (1)*ROW

51 doee2 $=0$.

do $40 \quad 1=1,7$

$a m=b(3,1)$

a) $1=-a 3(3,1)$

a.12 $=a 4(3, i)$

Ig=CW $(1) *$ RoW

40

$B F A=a m * \exp (-a 11 * \lg t 2)+(1 .-\operatorname{am}) \star \exp (-a 12 * g i g * t 2)$

dose2 =doee2+Dosewg (i) * exp $(-8 i g * t 2) * B F A$

If (dose2.1t.dose) go to 50

$t 2=1.01 * t 2$

$i t=1 t+1$

if(it.le.itmag) go to 51

igam $=0$

50

write $(8, *)$ 'Problem with Calculation of Gamma Shielding'

continue

f2=Dose2-Dose

if (1gam. og.0) return

$1 t=0$

$61 t \operatorname{tgam}=0.5 *(t 1+t 2)$

dosea $=0$.

do $41 \quad 1=1,7$

$a m=b(3, i)$

a.1 $1=-a 3(3,1)$

a $12=a 4(3, i)$

- Ig=GW (i) * ROW

$B F A=a m * \exp (-a 12 * a i g * \operatorname{tgam})+(1 .-a m) \star \exp (-a 12 * g i g * \operatorname{tgam})$

c

c recompute neutron shielding(ten) because $w$ also shield neutrons

$c$ see: if ten<0. program set ten to 0 . (le no need of $n$ shielding

$c$ because $w$ is enough also if ishin=0 (fluin lt prescribed fluout)

1f(1ten. og.1) ten=AA-BBB*tgam

if (ten. $1 t .0$.$) ten =0$.

if (iten.eg.i. and.ishin.eg.0) ten $=0$.

$\operatorname{ten}=\operatorname{sign}(i)$ *ten

$\operatorname{shin}=\exp (-\tan ) *(\operatorname{An}(1) * \exp (-\tan * A 1 n(1))+(1 .-\operatorname{An}(i)) * \exp (-\tan * A 2 n(1))$

Page B-48 


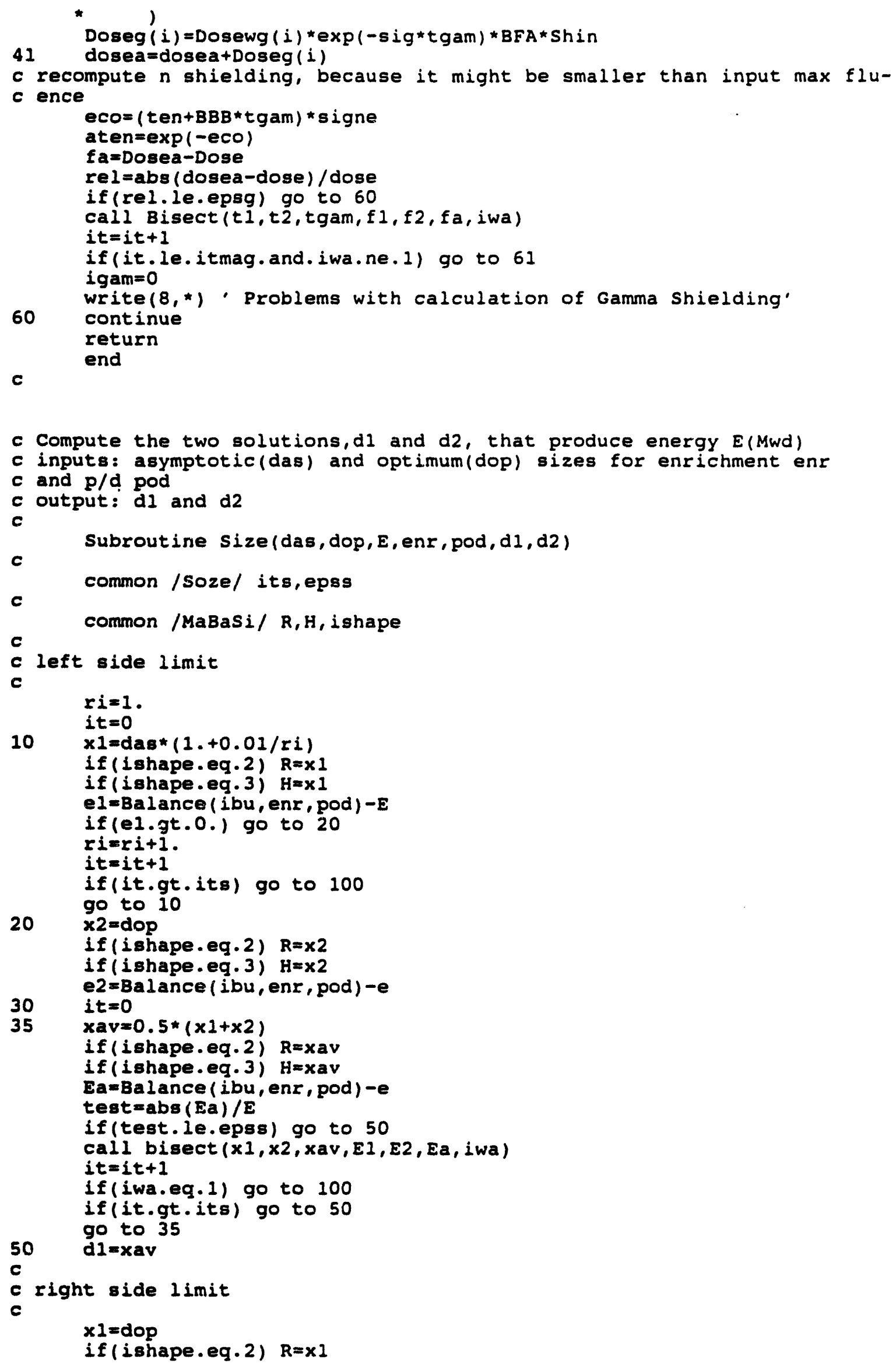




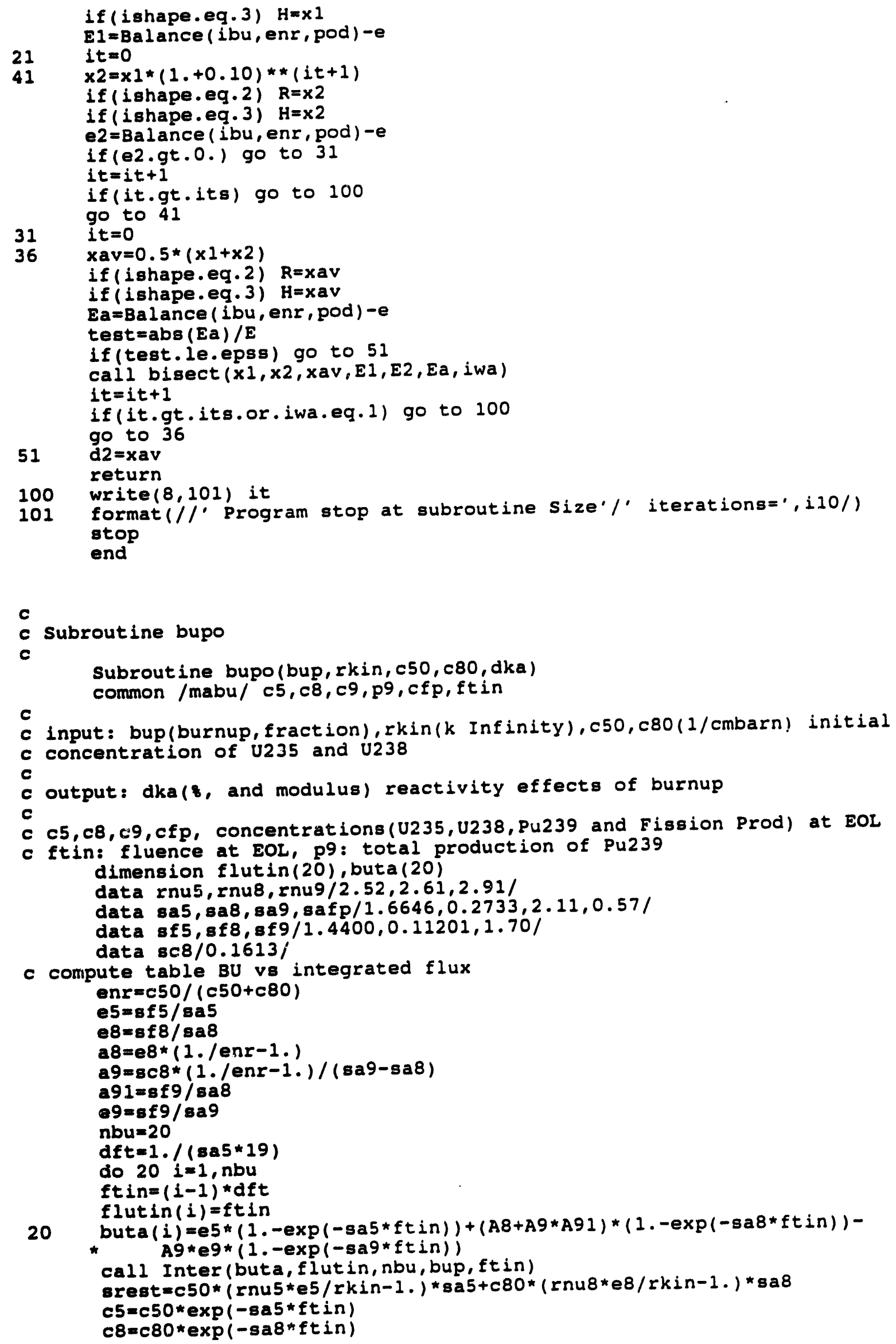


$c 9=(8 c 8 * c 80 /(s a 9-8 a 8)) *(\exp (-s a 8 * f t i n)-\exp (-s a 9 * f t i n))$

$\mathrm{p} 9=(\mathrm{cBO}-\mathrm{c} 8) * \mathrm{sc} 8 / \mathrm{sa} 8$

c cf $p=2 . *(c 50 * g f 5+c 80 * g f 8) * f t$ in cf $p=2 . *((c 50-c 5) * e 5+(c 80-c 8) * e 8+(p 9-c 9) * e 9)$

rkinbu $=($ rnu $5 * c 5 * s f 5+$ rnu $8 * c 8 * s f 8+r n u g * c 9 * s f 9) /$ 
INTERNAL DISTRIBUTION
1. C. E. Oliver
2. R. Cooper
3-7. F. C. Difilippo
8. R. S. Holcomb
9. G. E. Michaels
10. W. C. Jordan
11. R. B. Perez
12. R. T. Primm, III
13. J. P. Reiner

\author{
14. J. C. Turner \\ 15. R. C. Ward \\ 16-20. B. A. Worley \\ 21. EPMD Reports Office \\ 22-26. Laboratory Records Department \\ 27. Laboratory Records, ORNL-RC \\ 28. Document Reference Section \\ 29. Central Research Library \\ 30. ORNL Patent Section
}

\section{EXTERNAL DISTRIBUTION}

31. S. Anghaie, 202 NCS, University of Florida, Gainesville, FL 32611.

32. Gary Bennett, NASA Headquarter, Code RST, Washington, DC 20546.

33. Jeff George, NASA Lewis Research Center, MS AAC-2, 21000 Brookpark Road, Cleveland, OH 44135.

34. Nils J. Diaz, INSPI, University of Florida, Gainesville, FL 32611.

35. Dean Dobranovich, Sandia National Laboratory, P.O. Box 5800, Albuquerque, NM 87185-5800.

36-40. M. P. Doherty, NASA Lewis Research Center, MS-500/322, 21000 Brookpark Road, Cleveland, OH 44135.

41. Al Juhasz, NASA Lewis Research Center, MS 301-5, 21000 Brookpark Road, Cleveland, OH 44135.

42. A. C. Zuppero, DOE-INEL, P.O. Box 1625, MS-3413, Idaho Falls, ID 83415.

43. R. W. Brockett, Harvard University, Pierce Hall, 29 Oxford Street, Cambridge, MA 02138.

44. D. J. Dudziak, Department of Nuclear Engineering, 110B Burlington Engineering Labs, North Carolina State University, Raleigh, NC 27695-7909.

45. J. E. Leiss, Rt. 2, Box 142C, Broadway, VA 22815.

46. N. Moray, Department of Mechanical and Industrial Engineering, University of Illinois, 1206 West Green Street, Urbana, IL 61801.

47. M. F. Wheeler, Department of Mathematics, Rice University, P.O. Box 1892 , Houston, TX 77251.

48. Office of Assistant Manager for Energy Research and Development, Department of Energy, Oak Ridge Operations Office, P.O. Box 2008, Oak Ridge, TN 37831.

49-50. Office of Scientific and Technical Information, U.S. Department of Energy, P.O. Box 62, Oak Ridge, TN 37831.

51. Report Control, NASA Lewis Research Center, 21000 Brookpark Road, Mail Stop 60/1, Cleveland, $\mathrm{OH} 44135$.

52-53. Library, NASA Lewis Research Center, 21000 Brookpark Road, Mail Stop 60/3, Cleveland, $\mathrm{OH} 44135$.

54. Technology Utilization Office, NASA Lewis Research Center, 21000 Brookpark Road, Mail Stop 7/3, Cleveland, OH 44135.

55-79. NASA Center for Aerospace Information, ATTN: Accession Branch, P.O. Box 8757, Baltimore, MD 21240-0757. 


\begin{tabular}{|c|c|c|c|}
\hline \multicolumn{3}{|c|}{ REPORT DOCUMENTATION PAGE } & $\begin{array}{l}\text { Form Approved } \\
\text { OMB No. 0704-0188 }\end{array}$ \\
\hline \multicolumn{4}{|c|}{ 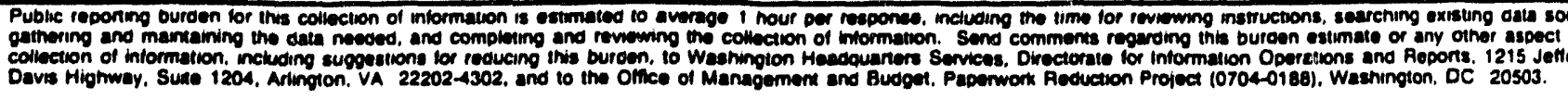 } \\
\hline 1. AGENCY USE ONLY (Leave Dlank) & $\begin{array}{l}\text { 2. REPOAT DATE } \\
\text { March } 1994\end{array}$ & & $\begin{array}{l}\text { DO DATES COVERED } \\
\text { or Report }\end{array}$ \\
\hline \multicolumn{3}{|c|}{$\begin{array}{l}\text { 4. TTLEAND SUBTITLE } \\
\text { Scoping Calculations of Power Sources for } \\
\text { Nuclear Electric Propulsion }\end{array}$} & 5. FUNDING NUMBERS \\
\hline \multicolumn{4}{|l|}{ Felix C. Difilippo } \\
\hline \multicolumn{3}{|c|}{$\begin{array}{l}\text { Oak Ridge National Laboratory } \\
\text { Engineering Physics and Mathematics Division } \\
\text { P.0. Box 2008, B1dg. } 6035 \\
\text { Oak Ridge, TN } 37831-6363\end{array}$} & $\begin{array}{l}\text { 8. PERFORMING OAGANIZATION } \\
\text { REPORT NUMBEA } \\
\text { ORNL/TM-12703 }\end{array}$ \\
\hline \multicolumn{3}{|c|}{$\begin{array}{l}\text { 9. SPONSORINGMOMITORING AGENCY MAMES(S) AND ADDAESS(ES) } \\
\text { National Aeronautics and Space Administration } \\
\text { Lewis Research Center } \\
21000 \text { Brookpark Road } \\
\text { Cleveland, OH } 44137\end{array}$} & $\begin{array}{l}\text { 10. SPONSOAING/MONITOAING } \\
\text { RGENCY REPORT NUMBER } \\
\text { NASA CR-191133 }\end{array}$ \\
\hline
\end{tabular}

\section{SUPPLEMENTARY NOTES}

Felix C. Difilippo, Oak Ridge National Laboratory, P.0. Box 2008, Oak Ridge, TN 37831-6363 (615) 574-6188.

\begin{tabular}{|l|l|}
\hline 120. DIBTRLBUTIONIAVALLABILTY STATEMENT & 12b. DISTRIBUTION CODE \\
Unclassified-Unlimited & \\
\hline
\end{tabular}

\section{ABSTAACT (MUOXImum 200 wordes)}

This technical memorandum describes models and calculational procedures to fully characterize the nuclear 1sland of power sources for nuclear electric propulsion. Two computer codes were written: one for the gas-cooled NERVA derivative reactor and the other for liquid metal-cooled fuel pin reactors. These codes are going to be interfaced by NASA with the balance of plant in order to making scoping calculations for mission analysis.

\section{BUEJECT TEAME}

Nuclear electrfc propulsion, Reactor modeling, Liquid metal cooled reactor, Gas cooled reactor

\begin{tabular}{|c|c|c|c|}
\hline $\begin{array}{l}\text { 17. 8ECUATY CLABSIFICATION } \\
\text { OF REPORT } \\
\text { Unclassif Ied }\end{array}$ & $\begin{array}{l}\text { 18. SECUATY CLABSIFICATION } \\
\text { OF THIS PAOE } \\
\text { UnClassified }\end{array}$ & $\begin{array}{l}\text { 19. SECUATY CLASSIFICATION } \\
\text { OF ADSTAACT } \\
\text { Unclassif } 1 \text { ed }\end{array}$ & $\begin{array}{l}\text { 20. LMITATION OF ABSTRACT } \\
\text { None }\end{array}$ \\
\hline
\end{tabular}



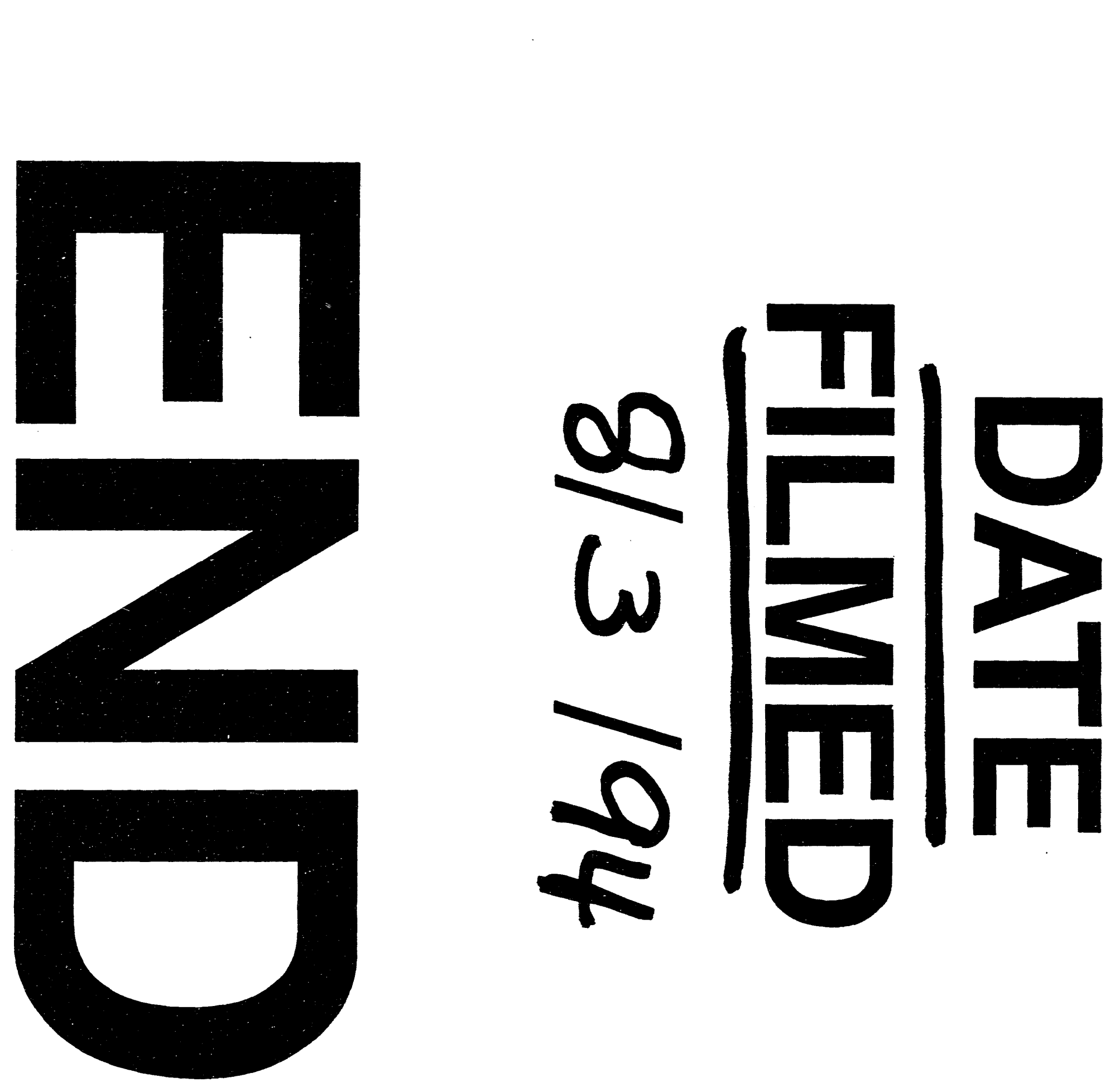
